\begin{abstract}
HADDAD, REDA NASSIF. 3-tier Service Level Agreement with automatic class upgrades. (Under the direction of Dr. Yannis Viniotis).

Tremendous efforts have been spent on devising mechanisms that would provide Quality of Service (QoS) needed by various applications, and network operators have spent a lot of resources trying to fit their networks with differentiated services capabilities. One of the Service Level Agreements (SLA) promising to sell these QoS services is the "triple play" SLA, bundling 3 classes of services targeting voice, data and video. In particular, circuit switched network operators envision the triple play SLA as essential to revenue maintenance, customer retention, and growth. It is their way, through the IP Multimedia Subsystem (IMS) standardization for example, to move all non-IP current and future services, such as voice, onto IP.

In this thesis, we propose a "3-tier SLA with automatic class upgrades", an enhancement to the triple play SLA, in that it automatically upgrades lower classes' packets to fill gaps or unused bandwidth in the upper classes. The proposed SLA incorporates a scalable solution to the reordering problem, caused by upgrading lower class-packets to upper classes; the solution does not require per flow state information. We provide a thorough analysis of the QoS performance in terms of goodput, losses and delay of both UDP and TCP sources and show that the proposed SLA maximizes the customer's utilization of the reserved and paid-for bandwidth by maximizing the utilization of the most expensive, better service, upper QoS classes, and provides much greater throughput than the proposed "triple play" model.
\end{abstract}




\title{
3-TIER SERVICE LEVEL AGREEMENT WITH AUTOMATIC CLASS UPGRADES
}

\author{
by \\ REDA NASSIF HADDAD
}

A dissertation submitted to the Graduate Faculty of

North Carolina State University

in partial fulfillment of the requirements for the Degree of

Doctor of Philosophy

\section{COMPUTER ENGINEERING}

\author{
Raleigh, NC
}

2006

\section{APPROVED BY:}




\section{Dedication}

To my parents, Nassif w Nehmat,

To my family, Doha, Mona, w Elias, And to all Lebanese, my compatriots.

"September $14^{\text {th }}$, 1982 was not the death of a dream.

It was the birth of a new revolution. A revolution $10452 \mathrm{Km}^{2}$ wide"

- Marechal - 


\section{Biography}

Reda N. Haddad was born in Hammana, Lebanon. He received the B.E. degree in Computer and Communication Engineering from the American University of Beirut (AUB), in 1998, and the M.S. degree in Computer Networking from North Carolina State University (NCSU), in 2000.

During 1999 - 2000, he was a Research Assistant in the Department of Electrical and Computer Engineering, at NCSU. During 2000 - 2006 he worked at Ericsson IP Infrastructure, Raleigh, NC, in Research and Development of router products covering several areas including DiffServ, MPLS and Forwarding Control Layer. He was also nominated as product Technical Coordinator and System Manager covering areas such as Router Architecture and Design. He is also the Editor of several Network Processing Forum Implementation Agreements including the MPLS SAPI, the IPv4 SAPI and the Next Hop FAPI. His research interests include Service Level Agreements, Quality of Service, optimal Router Design, Network Resiliency, and performance analysis of IP networks. 


\section{Acknowledgements}

I would like to express my sincerest gratitude to the chair of the advisory committee, my Professor, my Plato, my Idol, and my Friend Dr. Yannis Viniotis. Thank you for your patience (however, Pythagoras is Phoenician!!), for your wisdom (“Arak" is not to be taken as a shot!!), for your guidance (after 234 steps, we finally reached the summit... I am talking about l'Arc de Triomphe"), for your teachings (for all the very late night discussions about the Middle-East), for being there (and thus the name "Wine-iotis")...

I would also like to thank all the members of the advisory committee, Dr. Harry Perros, Dr. Michael Devetsikiotis, Dr. Mihail Sichitiu, and last but not least Dr. Rudra Dutta. It was a pleasure to be under your supervision and guidance. 


\section{Table of Content}

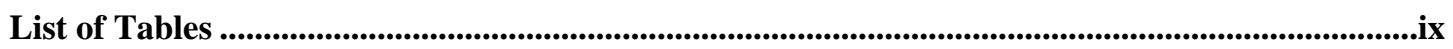

List of Figures.

....x

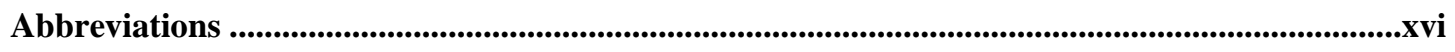

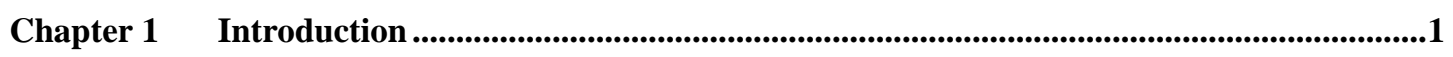

Chapter 2 SLA Overview............................................................................................................................

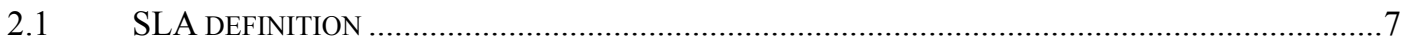

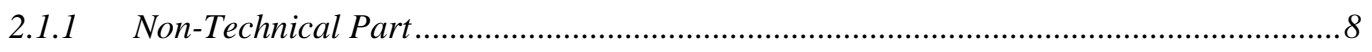

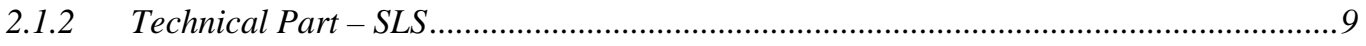

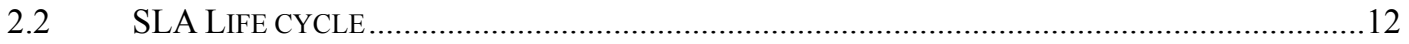

Chapter 3 SLA Research Areas ..........................................................................................................14

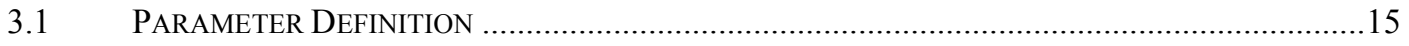

3.1.1 Standard Metrics/Parameters ……………...................................................................16

3.1.2 New Metrics/Parameters _........................................................................................16

3.1.3 Languages and Templates ......................................................................................18

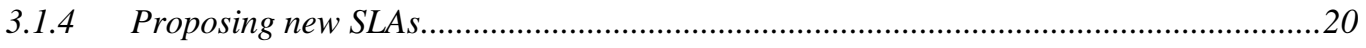

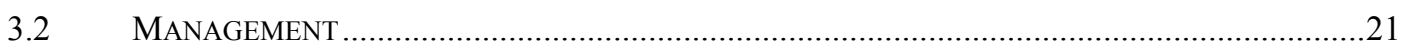

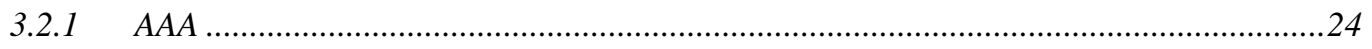

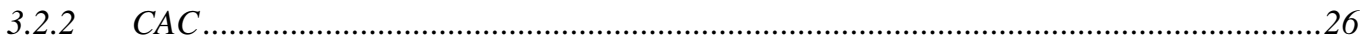

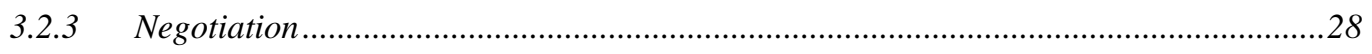

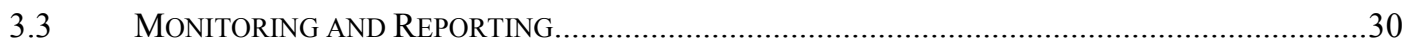

3.3.1 Tools and Techniques...........................................................................................

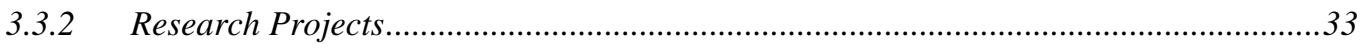

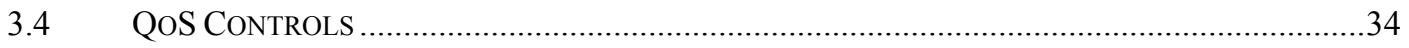


Chapter $4 \quad$ SLAs and Network Technologies .....................................................................................

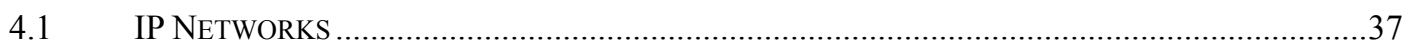

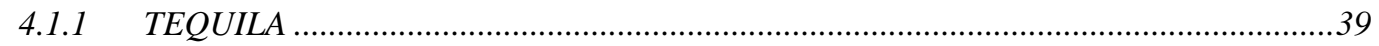

4.1.2 AQUILA .

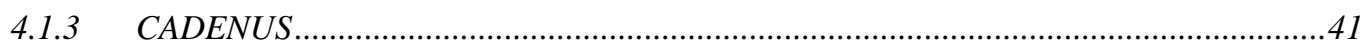

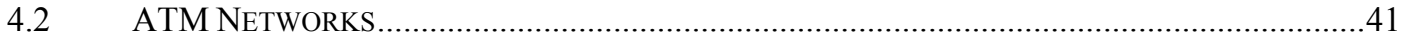

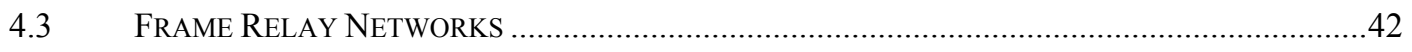

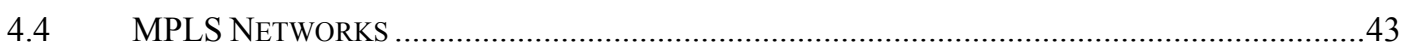

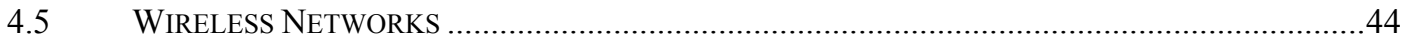

Chapter 5 3-Tier SLA with Automatic Class Upgrades.......................................................................47

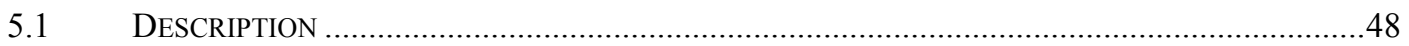

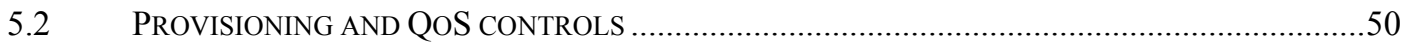

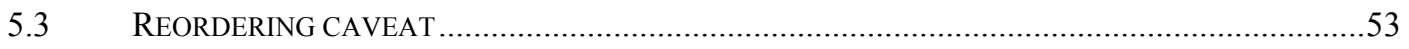

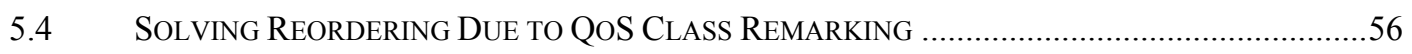

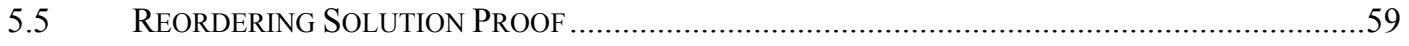

Chapter 6 Experimental Results - 3-Tier SLA Performance ........................................................63

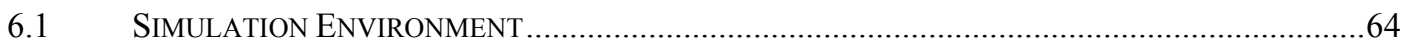

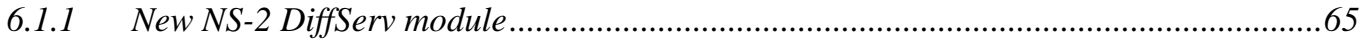

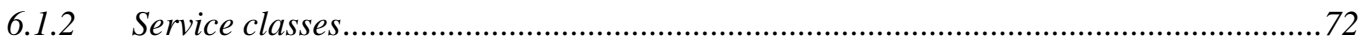

6.1.3 General Information on Results ................................................................................

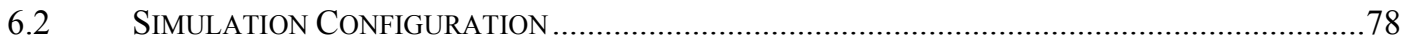

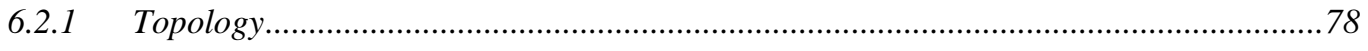

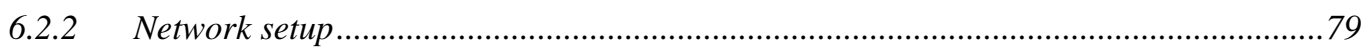

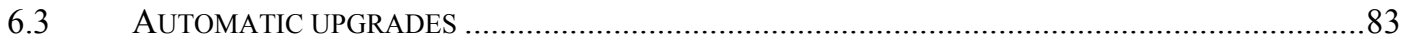

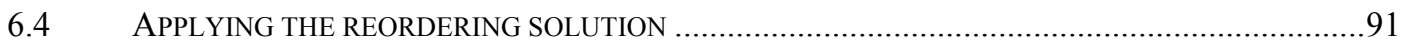

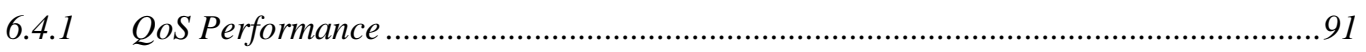

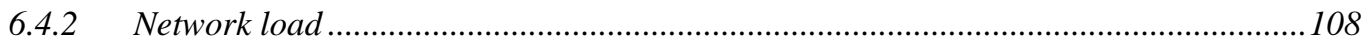




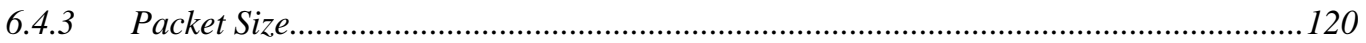

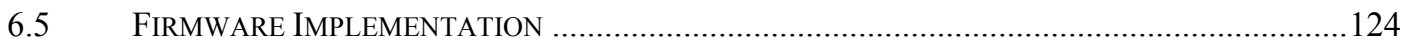

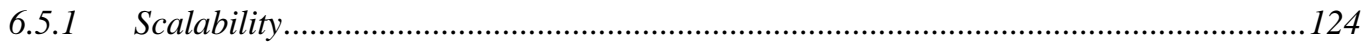

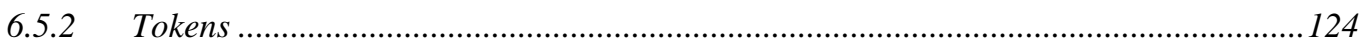

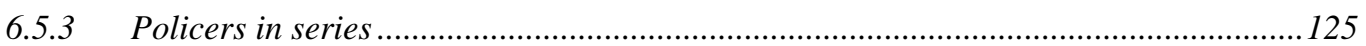

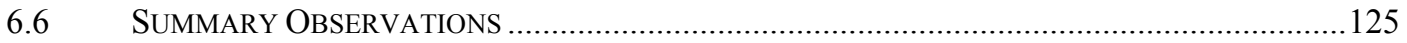

Chapter $7 \quad$ Experimental Results - Bursty Traffic........................................................................126

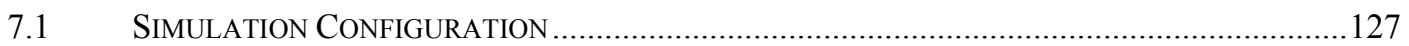

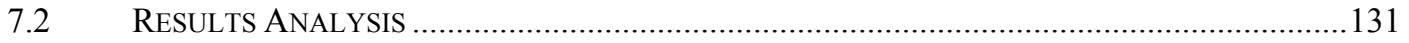

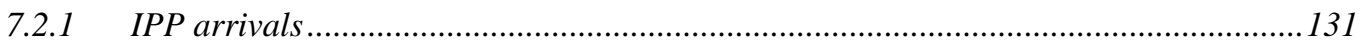

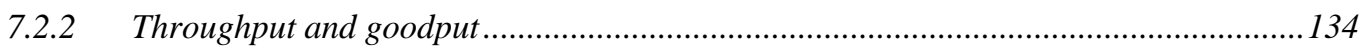

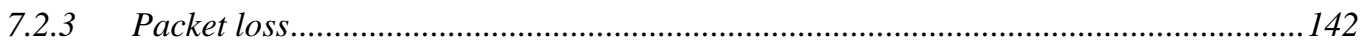

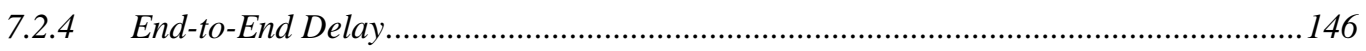

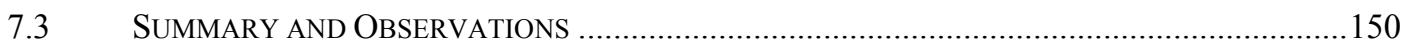

Chapter 8 Experimental Results - TCP Highlights..........................................................................152

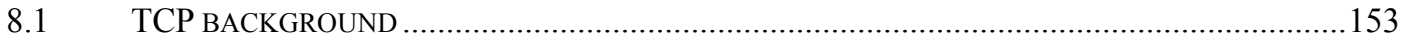

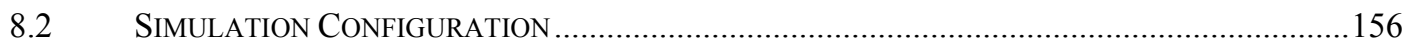

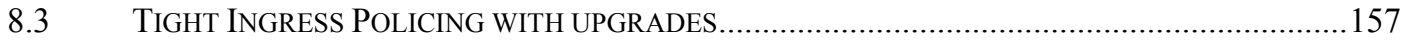

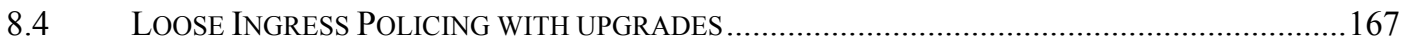

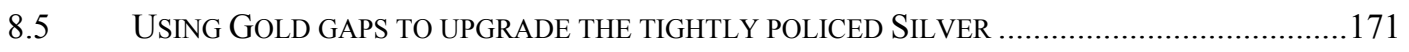

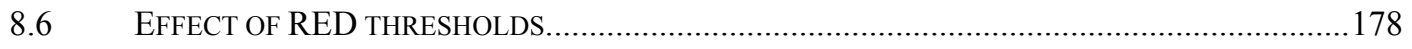

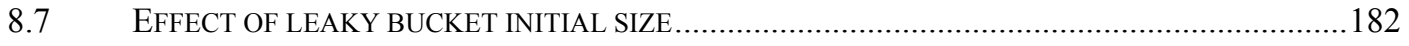

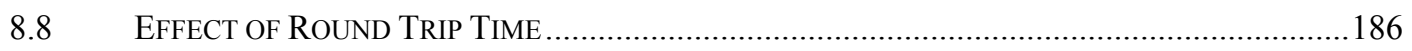

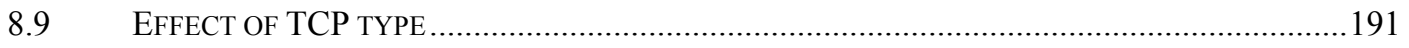

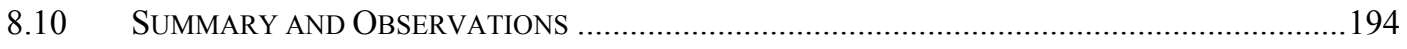

Chapter 9 Experimental Results - Generalizing into N-Class SLA...........................................196

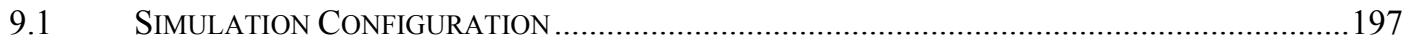




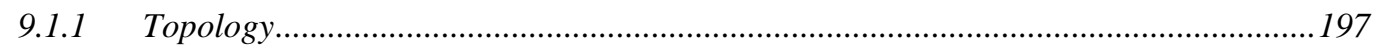

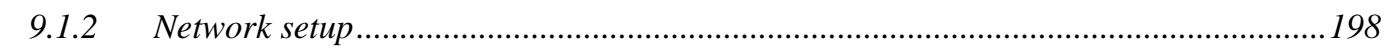

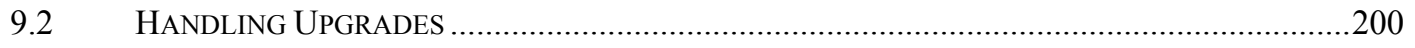

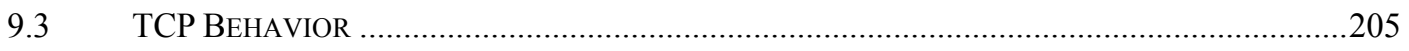

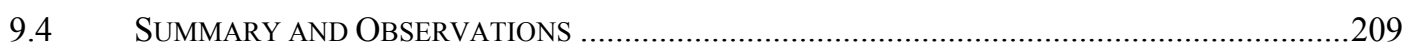

Chapter 10 Conclusion and Future Research ................................................................................210

Bibliography ....................................................................................................................................215 


\section{List of Tables}

TABLE 1 APPLICATIONS AND THEIR QOS REQUIREMENTS [55] ......................................................

TABLE 2 RESEARCH PROJECTS RELATED TO SLA MEASUREMENT ………........................................

TABLE 3 3GPP QOS CLASSES CLASSIFICATION [56] .......................................................................... 44

TABLE 4 3-TIER SLA WITH AUTOMATIC UPGRADES …....................................................................49

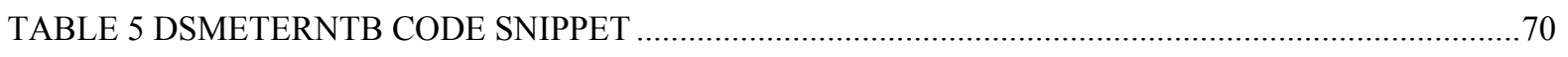

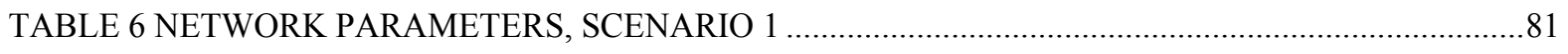

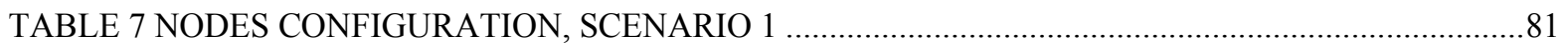

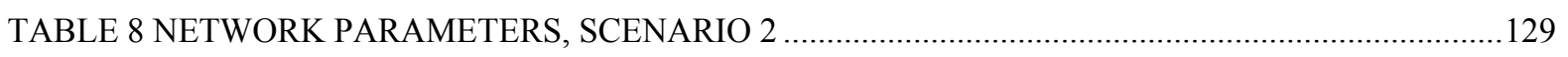

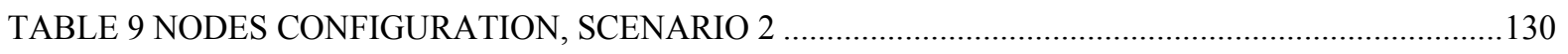

TABLE 10 NETWORK PARAMETERS, 8-CLASSES SCENARIO ……................................................... 199

TABLE 11 NODES CONFIGURATION, 8 CLASSES SCENARIO …….................................................20 


\section{List of Figures}

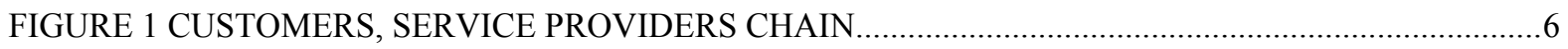

FIGURE 2 QOS CONTROLS FOR 3-TIER SLA WITH CLASS UPGRADES .................................................50

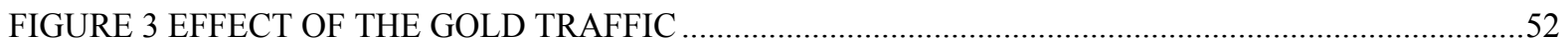

FIGURE 4 EFFECT OF THE GOLD RATE ON OTHER CLASSES ………...............................................53

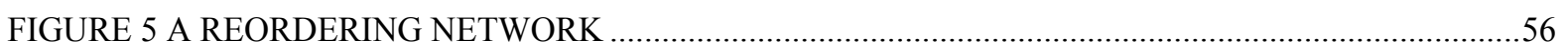

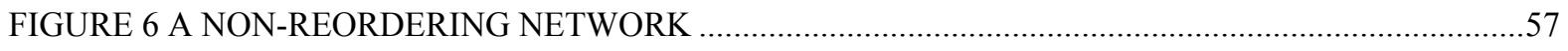

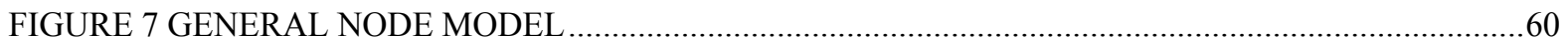

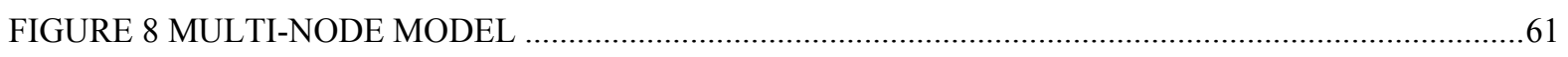

FIGURE 9 INGRESS EDGE NODE DSQUEUE MODEL .........................................................................6

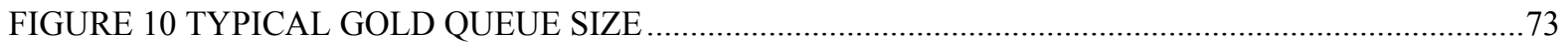

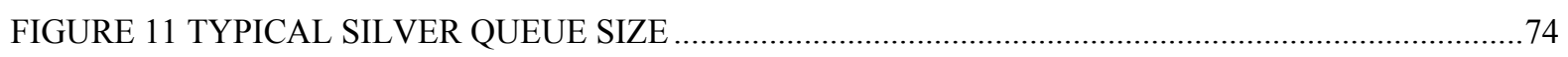

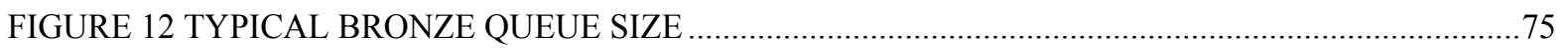

FIGURE 13 TYPICAL WORST CASE END-TO-END DELAY CDF ………….........................................

FIGURE 14 TYPICAL WORST CASE PER HOP FORWARDING RATE …....................................................76

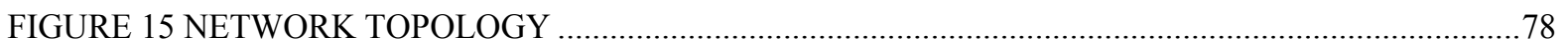

FIGURE 16 SILVER AND BRONZE THROUGHPUT VERSUS GOLD RATE ............................................84

FIGURE 17 SILVER AND BRONZE UPGRADE RATES VERSUS GOLD RATE .....................................84

FIGURE 18 GOLD AND BRONZE THROUGHPUT VERSUS SILVER RATE...............................................85

FIGURE 19 GOLD AND BRONZE UPGRADE RATES VERSUS SILVER RATE .......................................86

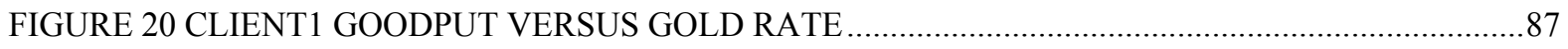

FIGURE 21 CLIENT1 GOODPUT AND THROUGHPUT VERSUS GOLD RATE ….......................................87

FIGURE 22 CLIENT1 REORDERED PACKETS VERSUS GOLD RATE .................................................89

FIGURE 23 GOLD, SILVER AND BRONZE GOODPUT VERSUS SILVER RATE..................................90

FIGURE 24 GOLD, SILVER AND BRONZE REORDERING VERSUS SILVER RATE .............................90

FIGURE 25 DS, SLAR AND SLA SILVER THROUGHPUT VERSUS GOLD RATE ..................................92

FIGURE 26 DS, SLAR, SLA BRONZE THROUGHPUT VERSUS GOLD RATE.........................................92 
FIGURE 27 DS, SLAR, SLA SILVER GOODPUT VERSUS GOLD RATE

FIGURE 28 DS, SLAR, SLA BRONZE GOODPUT VERSUS GOLD RATE ..........................................94

FIGURE 29 DS, SLAR, SLA BRONZE GOODPUT VERSUS SILVER RATE........................................95

FIGURE 30 DS THROUGHPUT AND GOODPUT VERSUS GOLD RATE ...........................................96

FIGURE 31 SLAR THROUGHPUT AND GOODPUT VERSUS GOLD RATE ........................................96

FIGURE 32 SLA THROUGHPUT AND GOODPUT VERSUS GOLD RATE .......................................97

FIGURE 33 SLA LOSSES VERSUS GOLD RATE ........................................................................

FIGURE 34 SLA INGRESS POLICING DROPS VERSUS GOLD RATE...............................................98

FIGURE 35 DS, SLAR, SLA SILVER PACKET LOSS VERSUS GOLD RATE .......................................99

FIGURE 36 DS, SLAR, SLA SILVER INGRESS DROP VERSUS GOLD RATE ....................................100

FIGURE 37 DS, SLAR, SLA BRONZE PACKET LOSS VERSUS GOLD RATE ......................................100

FIGURE 38 DS, SLAR, SLA BRONZE INGRESS DROP VERSUS GOLD RATE .................................101

FIGURE 39 DS, SLAR, SLA BRONZE PACKET LOSS VERSUS SILVER RATE ..................................102

FIGURE 40 DS, SLAR, SLA BRONZE INGRESS DROP VERSUS SILVER RATE .................................102

FIGURE 41 DS, SLAR, SLA END-TO-END GOLD DELAY VERSUS GOLD RATE................................104

FIGURE 42 DS, SLAR, SLA END-TO-END GOLD DELAY VERSUS SILVER RATE...........................105

FIGURE 43 DS, SLAR, SLA END-TO-END SILVER DELAY VERSUS GOLD RATE............................106

FIGURE 44 DS, SLAR, SLA END-TO-END SILVER DELAY VERSUS SILVER RATE .........................106

FIGURE 45 DS, SLAR, SLA END-TO-END BRONZE DELAY VERSUS GOLD RATE ...........................107

FIGURE 46 DS, SLAR, SLA END-TO-END BRONZE DELAY VERSUS SILVER RATE.......................108

FIGURE 47 DS, SLAR, SLA SILVER GOODPUT VERSUS BACKGROUND GOLD RATE ....................109

FIGURE 48 DS, SLAR, SLA BRONZE GOODPUT VERSUS BACKGROUND GOLD RATE .................109

FIGURE 49 SLAR SILVER AND BRONZE REORDERING VERSUS BACKGROUND GOLD RATE.....110

FIGURE 50 SLAR SILVER AND BRONZE LOSSES VERSUS BACKGROUND GOLD RATE ...............111

FIGURE 51 SLAR N_N2 SILVER QUEUE SIZE FOR CBR7=71303BPS .........................................112

FIGURE 52 SLAR N_N2 SILVER QUEUE SIZE FOR CBR7=855636BPS ...........................................112

FIGURE 53 SLAR N_N2 SILVER QUEUE RED LOSSES FOR CBR7=71303BPS .................................113

FIGURE 54 SLAR N_N2 SILVER QUEUE RED LOSSES FOR CBR7=855636BPS ..............................113 
FIGURE 55 SILVER (LEFT) AND BRONZE (RIGHT) N_N2 QUEUE SIZES AT CBR7=213909 BPS .......114 FIGURE 56 SILVER (LEFT) AND BRONZE (RIGHT) N_N2 QUEUE SIZES AT CBR7=356515 BPS ......115 FIGURE 57 SILVER (LEFT) AND BRONZE (RIGHT) N_N2 QUEUE SIZES AT CBR7=499121 BPS ......115 FIGURE 58 DS, SLAR, SLA GOLD AVERAGE DELAY VERSUS BACKGROUND GOLD RATE..........116 FIGURE 59 DS, SLAR, SLA SILVER AVERAGE DELAY VERSUS BACKGROUND GOLD RATE .......116 FIGURE 60 DS, SLAR, SLA BRONZE AVERAGE DELAY VERSUS BACKGROUND GOLD RATE .....117 FIGURE 61 DS, SLAR, SLA SILVER GOODPUT AT LOW NETWORK LOAD VERSUS GOLD RATE.118 FIGURE 62 SLAR REORDERING WITH HIGH AND LOW NETWORK LOAD VERSUS GOLD RATE.119 FIGURE 63 DS, SLAR, SLA BRONZE GOODPUT AT LOW NETWORK LOAD VERSUS GOLD RATE120 FIGURE 64 DS, SLAR, SLA SILVER GOODPUT VERSUS PACKET SIZE ..............................................121 FIGURE 65 DS, SLAR, SLA BRONZE GOODPUT VERSUS PACKET SIZE ............................................121 FIGURE 66 SLAR REORDERING VERSUS PACKET SIZE .................................................................122 FIGURE 67 DS, SLAR, SLA END-TO-END SILVER DELAY VERSUS PACKET SIZE ..........................123

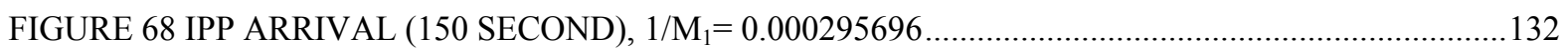

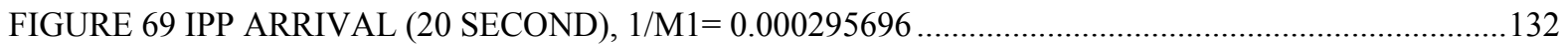
FIGURE 70 IPP ARRIVALS WITH DIFFERENT AVERAGE RATES ......................................................133 FIGURE 71 ON AND OFF DISTRIBUTIONS FOR 1/M $\mathrm{M}_{1}=0.000295696,1 / \mathrm{M}_{2}=0.023655735 \ldots \ldots \ldots \ldots \ldots \ldots . . . . .133$ FIGURE 72 PACKET SIZE DISTRIBUTION DURING THE ON PERIOD (AVERAGE = 500 BYTES) $\ldots . .134$

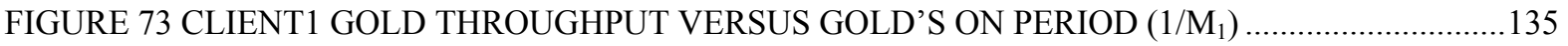
FIGURE 74 CLIENT1 GOLD AND SILVER THROUGHPUT VERSUS GOLD'S ON PERIOD $\left(1 / \mathrm{M}_{1}\right) \ldots . . .136$ FIGURE 75 CLIENT1 SILVER AND BRONZE THROUGHPUT VERSUS GOLD’S ON PERIOD $\left(1 / \mathrm{M}_{1}\right) .137$ FIGURE 76 CLIENT1 GOLD THROUGHPUT AND SILVER UPGRADES VERSUS GOLD'S ON PERIOD $\left(1 / \mathrm{M}_{1}\right)$ 137

FIGURE 77 CLIENT1 SILVER THROUGHPUT AND BRONZE UPGRADES VERSUS GOLD'S ON

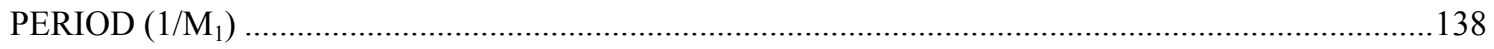

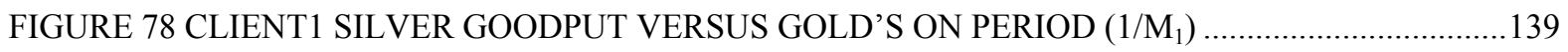
FIGURE 79 CLIENT1 BRONZE GOODPUT VERSUS GOLD'S ON PERIOD $\left(1 / \mathrm{M}_{1}\right)$. 139 FIGURE 80 CLIENT1 SILVER AND BRONZE REORDERING VERSUS GOLD’S ON PERIOD $\left(1 / \mathrm{M}_{1}\right) . .140$ 
FIGURE 81 CLIENT1 SILVER THROUGHPUT AND GOODPUT VERSUS GOLD’S ON PERIOD (1/M 1 )

FIGURE 82 CLIENT1 BRONZE THROUGHPUT AND GOODPUT VERSUS GOLD’S ON PERIOD (1/M $\left.{ }_{1}\right)$

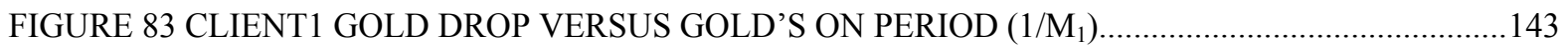

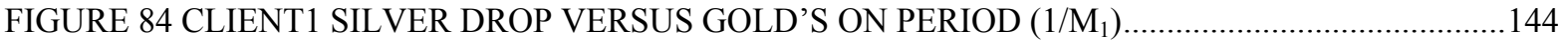

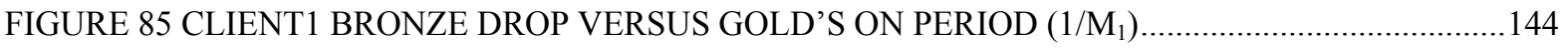

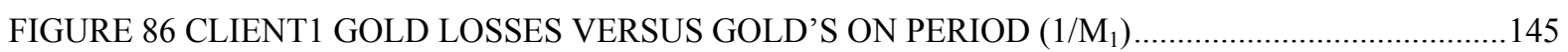

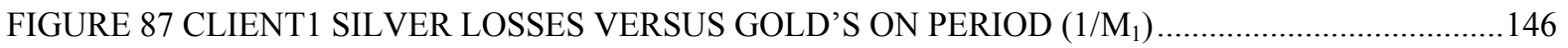

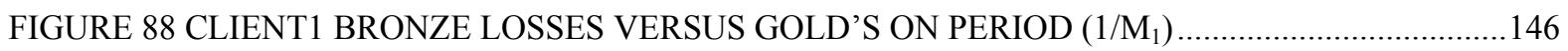

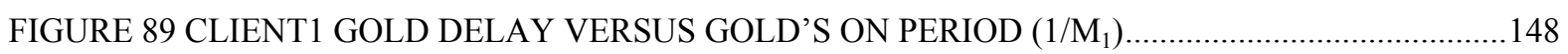

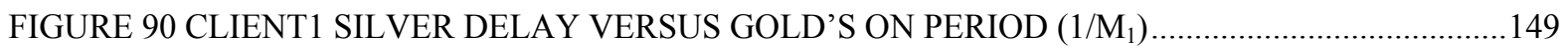

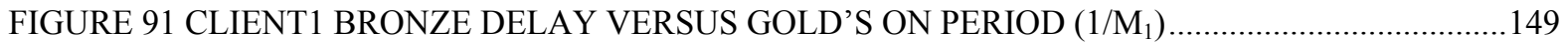

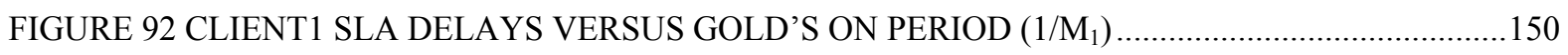

FIGURE 93 SILVER TCP GOODPUT VERSUS SILVER BACKGROUND RATE .....................................158

FIGURE 94 SILVER TCP AVERAGE FORWARD DELAY VERSUS SILVER BACKGROUND RATE...160

FIGURE 95 SILVER TCP INGRESS DROP RATE VERSUS SILVER BACKGROUND RATE ..................161

FIGURE 96 SILVER TCP 3+ DUPLICATE ACK COUNT VERSUS SILVER BACKGROUND RATE......165

FIGURE 97 SILVER TCP GOODPUT VERSUS SILVER BACKGROUND RATE ......................................168

FIGURE 98 SILVER TCP UPGRADES VERSUS SILVER BACKGROUND RATE ..................................169

FIGURE 99 SILVER TCP AVERAGE FORWARD DELAY VERSUS SILVER BACKGROUND RATE...169 FIGURE 100 SILVER TCP 3+ DUPLICATE ACK COUNT VERSUS SILVER BACKGROUND RATE....170 FIGURE 101 SILVER TCP GOODPUT VERSUS SILVER BACKGROUND RATE ..................................172 FIGURE 102 SILVER TCP 3+ DUPLICATE ACK COUNT VERSUS SILVER BACKGROUND RATE....173 FIGURE 103 SILVER TCP AVERAGE FORWARD DELAY VERSUS SILVER BACKGROUND RATE.174 FIGURE 104 GOLD, SILVER AND BRONZE SLA TCP GOODPUT VERSUS SILVER BACKGROUND RATE 175 FIGURE 105 BRONZE TCP GOODPUT VERSUS SILVER BACKGROUND RATE 176 
FIGURE 106 BRONZE TCP 3+ DUPLICATE ACK COUNT VERSUS SILVER BACKGROUND RATE..177 FIGURE 107 BRONZE TCP AVERAGE FORWARD DELAY VERSUS SILVER BACKGROUND RATE .178

FIGURE 108 SILVER TCP GOODPUT VERSUS RED MIN THRESHOLD ...............................................179 FIGURE 109 SILVER BACKGROUND LOSS RATE VERSUS RED MIN THRESHOLD ...........................181 FIGURE 110 SILVER AND BRONZE UPGRADE RATES VERSUS RED MIN THRESHOLD .................181 FIGURE 111 3+ DUPLICATE ACKS VERSUS RED MIN THRESHOLD …………...................................182 FIGURE 112 GOLD TCP GOODPUT VERSUS THE GOLD CBS (PACKET SIZE = 1,000B) ...................183 FIGURE 113 GOLD TCP GOODPUT VERSUS THE GOLD CBS (PACKET SIZE = 1,500B) ...................184 FIGURE 114 SILVER TCP GOODPUT VERSUS THE GOLD CBS (PACKET SIZE $=1,500 B) \ldots \ldots \ldots \ldots \ldots \ldots . . .185$ FIGURE 115 GOLD TCP GOODPUT VERSUS THE PER LINK PROPAGATION DELAY .......................187 FIGURE 116 MAX THEORETICAL TCP GOODPUT VERSUS THE PER LINK PROPAGATION DELAY 188

FIGURE 117 GOLD INGRESS DROP RATE DUE TO POLICING VERSUS THE PER LINK PROPAGATION DELAY. .188

FIGURE 118 SILVER TCP GOODPUT VERSUS THE PER LINK PROPAGATION DELAY ....................190 FIGURE 119 SILVER TCP UPGRADE RATE VERSUS THE PER LINK PROPAGATION DELAY ..........190 FIGURE 120 SLA GOLD TCP GOODPUT VERSUS SILVER BACKGROUND RATE .............................192 FIGURE 121 SLA SILVER TCP GOODPUT VERSUS SILVER BACKGROUND RATE............................192 FIGURE 122 SLA BRONZE TCP GOODPUT VERSUS SILVER BACKGROUND RATE.........................193 FIGURE 123 SLAR SILVER TCP GOODPUT VERSUS SILVER BACKGROUND RATE .........................193 FIGURE 124 SLAR BRONZE TCP GOODPUT VERSUS SILVER BACKGROUND RATE .......................194

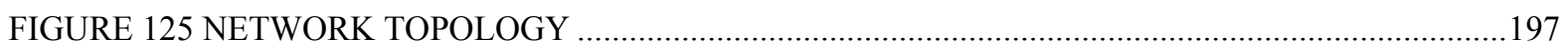
FIGURE 126 CLIENT1 P1 THROUGH P5 GOODPUT VERSUS P5 THROUGHPUT ................................201 FIGURE 127 CLIENT1 P5 THROUGH P8 GOODPUT VERSUS P5 THROUGHPUT ................................202 FIGURE 128 CLIENT1 P4 THROUGH P8 UPGRADES RATE VERSUS P5 THROUGHPUT ...................202 FIGURE 129 CLIENT1 P4 THROUGH P8 PACKET LOSSES VERSUS P5 THROUGHPUT......................203 FIGURE 130 CLIENT1 P5 THROUGH P8 GOODPUT VERSUS P5 THROUGHPUT ................................204 
FIGURE 131 CLIENT1 P5 THROUGH P8 GOODPUT VERSUS P5 THROUGHPUT ................................204

FIGURE 132 CLIENT1 P5 THROUGH P8 PACKETS REORDERED VERSUS P5 THROUGHPUT ...........205

FIGURE 133 CLIENT2 P1 THROUGH P5 GOODPUT VERSUS P6 BACKGROUND RATE .....................206

FIGURE 134 CLIENT2 P1 THROUGH P5 UPGRADE RATES VERSUS P6 BACKGROUND RATE........207

FIGURE 135 CLIENT2 P1 THROUGH P5 END-TO-END DELAY VERSUS P6 BACKGROUND RATE .207

FIGURE 136 CLIENT2 P5 THROUGH P8 GOODPUT VERSUS P6 BACKGROUND RATE .....................208

FIGURE 137 CLIENT2 P5 THROUGH P8 3+ DUPLICATE ACKS VERSUS P6 BACKGROUND RATE.208

FIGURE 138 CLIENT2 P5 THROUGH P8 UPGRADE RATES VERSUS P6 BACKGROUND RATE........209 


\section{Abbreviations}

3GPP Third Generation Partnership Project

AAA Authentication, Authorization and Accounting

BA Behavior Aggregate

CAC Connection Admission Control

CIR Committed Information Rate

DDR Data Delivery Ratio

DSCP Differentiated Services Code Point

EF $\quad$ Expedited Forwarding

FDR Frame Delivery Ratio

IETF Internet Engineering Task Force

MF $\quad$ Multi-Field (Classifier)

MIB Management Information Base

MRTG Multi Router Traffic Grapher

PIB Policy Information Base

PVC Permanent Virtual Circuit

RMON Remote Monitoring

SLA Service Level Agreement

SLI Service Level Indication

SLS Service Level Specification

TOS Type Of Service

UML Unified Modeling Language

UMTS Universal Mobile Telecommunications System 
VPN Virtual Private Network

WAN Wide Area Network

WRT Web Response Time

WRR Weighted Round Robin 


\section{Chapter 1}

\section{Introduction}

The development of network applications demanding service guarantees, such as voice and video communications, has yielded a tremendous effort for the definition, the specification and in some cases the standardization of the notion of Quality of Service (QoS) over various network technologies (ATM, IP, MPLS, etc.). Such applications rely on the deployment of value-added services offered by Service Providers. Because the subscription to such service offerings implies the definition of a contractual agreement, called Service Level Agreement (SLA), between the customer and the corresponding Service Provider, the level of quality associated with the services provided will be based upon a set of QoS parameters (such as delay, throughput, loss, etc.) both the customer and the provider have to agree upon.

The specification of the actual agreement, i.e. the SLA, includes the definition and the values of some measurable technical parameters defining the quality associated with the negotiated service that both the customer and provider can monitor throughout the life time of the SLA to make sure the traffic under contract is offered the paid-for service. 
Many SLA related research papers can be found in the literature, mainly classified into four areas. The first area covers defining new, or standardizing suggested QoS metrics or parameters involved in SLAs. The second area is concerned with managing the resources associated with the provision of SLAs, along with billing and accounting. The third area deals with monitoring and reporting the quality associated with the traffic defined by a certain SLA in order to verify the services under agreement. The fourth area encompasses the mapping of the Service Level Specification (the technical parameters such as a delay bound, a guaranteed throughput, etc.) described in an SLA to QoS controls, mechanisms or components (such as schedulers, queues, policers, routing algorithms, etc.) needed in providing the specification defined in such SLA.

Concerning the first area, several new SLAs have been suggested, each focusing on a specific problem or set of problems. In particular, in today's world, circuit-switched network operators are facing unprecedented challenges. Traditional sources of revenue are under attack; voice revenues are shrinking in both business and consumer markets. Moreover, subscribers are being lured away with aggressive pricing from emerging providers. Telecom operators are reacting with their own innovative voice solutions, often based on voice over IP (VoIP). A new suggested SLA called "triple play" bundling 3 classes of services targeting voice, data, and video is emerging as the winning combination. These new services are viewed as being essential to revenue maintenance, customer retention, and growth. In essence, the ultimate goal of triple play is to move all current and future services onto IP: data, voice and video.

In this thesis, we propose a "3-tier SLA with automatic class upgrades", an enhancement to the triple play SLA, in that it automatically upgrades lower classes' packets 
to fill gaps or unused bandwidth in the upper classes. If a customer pays for a "triple-play" SLA, why not fully use all the paid-for bandwidth? The proposed SLA incorporates a solution to the reordering problem caused by upgrading lower class packets to upper classes. The reordering problem is not peculiar to the 3-tier SLA but rather a general problem associated with SLAs or QoS technologies (such as DiffServ) that make use of packet remarking (upgrading or downgrading).

The thesis is organized as follows. We start by giving an overview on SLAs, defining the technical and non-technical terms of SLAs, and describing the SLA life cycle in Chapter 2. We then present in Chapter 3 the four main SLA research areas: 1. Parameter and SLA Definitions, 2. Management, 3. Monitoring and Reporting, and 4. QoS Controls Mapping. In Chapter 4, we depict the SLA applications in IP as well as legacy networks. In Chapter 5, we define the "3-tier SLA with automatic class upgrades" SLA, we suggest a set of QoS components that could provision the SLA, and we provide a solution, along with its proof, to the reordering problem due to upgrades. In Chapter 6, we present a detailed analysis of the SLA for Constant Bit Rate (CBR) traffic in regards to QoS performance using metrics such as throughput, delays, and packet losses, and comparing the SLA performance to the "triple play with no upgrades" and "triple play with upgrades but no reordering solution" through experimentation and simulations using the Network Simulator, NS-2. In Chapter 7, we continue the QoS performance analysis of the SLA focusing on bursty traffic sources using Interrupted Poisson Process (IPP). In Chapter 8, we highlight the SLA TCP-related issues including the effect of tight policing, the effect of the Committed Burst Size (CBS), the effect of the Round Trip Time, etc., and analyze the SLA QoS behavior under TCP-traffic. In Chapter 9, we generalize the "3-tier" SLA into an "N-tier" SLA by demonstrating the 
behavior for $\mathrm{N}=8$. Finally, we present a conclusion of our work in Chapter 10 and suggest several paths for future research. 


\section{Chapter 2}

\section{SLA Overview}

A Service Level Agreement is a contract between Service Providers and customers that specifies in measurable terms what services the Service Provider will furnish and what penalties the Service Provider will pay if s/he cannot meet the committed goals. Service Providers are companies that provide communications and/or data services as a business. They may operate networks, or integrate services of other providers to deliver a total service to their customers. Customers, on the other hand, are companies, organizations or individuals that make use of communications and/or data services provided by a Service Provider. Service Providers can be customers of other service providers as depicted in the value chain of service provisioning as shown in Figure 1.

Although there has been enormous amount of research in designing mechanisms for delivering QoS, its applications have been limited due to the missing link between QoS, SLA and pricing. Current pricing policies are in practice very simplistic (fixed price per unit capacity) and the corresponding SLAs provide very limited QoS options. This leads to provisioning based on peak load, underutilization of resources, and high costs. 


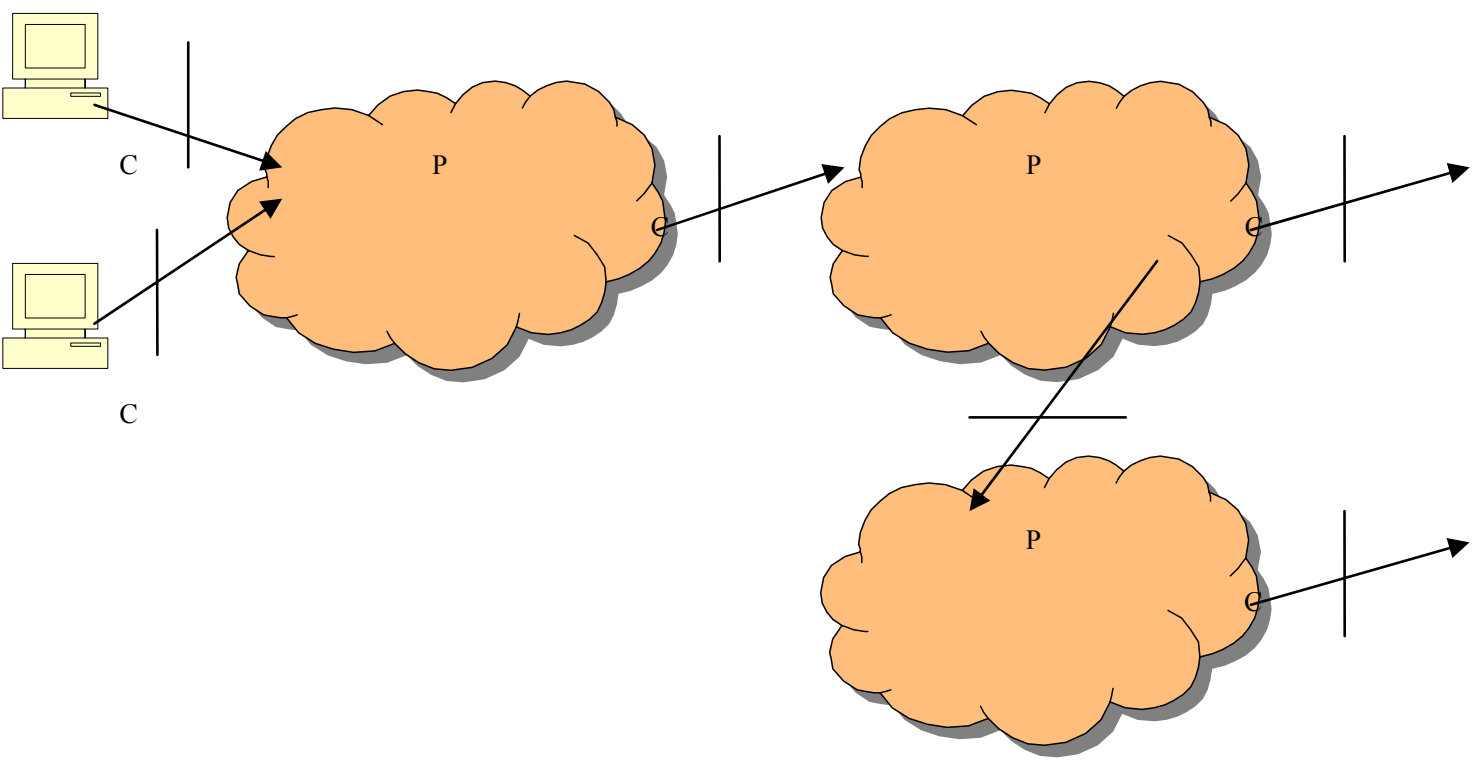

Figure 1 Customers, Service Providers chain

Network applications, such as file transfer, web browsing, live videoconferencing, etc. vary in their QoS requirements as shown inTable 1 [55] (a similar table is provided in [56]). A long file transfer needs a high throughput and low packet loss but is not very sensitive to delay and jitter. Live videoconferencing also needs high throughput but, on the other hand, is sensitive to both delay and jitter [55]. It is these differences that must be considered in writing the SLAs between providers and customers.

Table 1 Applications and their QoS Requirements [55]

\begin{tabular}{|l|l|l|l|l|}
\hline \multirow{2}{*}{ Traffic Type } & \multicolumn{4}{|c|}{ Requirements } \\
\cline { 2 - 5 } & Bandwidth & Loss & Delay & Jitter \\
\hline Voice & Very low & Medium & High & High \\
\hline E-Commerce & Low & High & High & Low \\
\hline Transactions & Low & High & High & Low \\
\hline E-mail & Low & High & Low & Low \\
\hline Telnet & Low & High & Medium & Low \\
\hline Casual Browsing & Low & Medium & Medium & Low \\
\hline Serious Browsing & Medium & High & High & Low \\
\hline File transfers & High & Medium & Low & Low \\
\hline Video Conferencing & High & Medium & High & High \\
\hline Multicasting & High & High & High & High \\
\hline
\end{tabular}


SLA requirements, depending on the side, do not deal with the same center of interest and are sometimes contradictory (e.g., a customer may want a performance report on the delivered QoS and the Service Provider does not want to provide this information in case of degradation of QoS). For the customer, the requirements deal with the definition, validation, guarantees, control, reporting, and discount in case of service degradation. For Service Providers, the requirements are to fulfill customer requirements, to profit from offered services, to reduce cost, and to self-differentiate from competitors.

Section 2.1 defines the various components of an SLA and section 2.2 gives an overview of the SLA life cycle.

\subsection{SLA definition}

A Service Level Agreement (SLA) is an arrangement between a customer and a provider describing technical and non-technical characteristics of a service including QoS requirements and the related set of metrics with which provision of these requirements is being measured. It is "an explicit statement of the expectations and obligations that exist in a business relationship between two organizations: the service provider and the customer". The content of an SLA varies depending on the service offered and incorporates the elements and attributes required for the particular negotiation. In general, it includes: A description of the service that is to be provided, the expected performance, a detailed procedure of handling problems, a procedure for monitoring and reporting the service level, the consequences of the service provider not meeting the agreed service, an end-point description of the contractors (e.g., information on customer/provider location and facilities), contractual statements (e.g., 
start date, duration of agreement, charging classes), and Service Level Specification (SLS) i.e., the technical QoS description and the associated metrics.

There is a distinction between horizontal and vertical SLAs. Horizontal SLAs govern the interaction between coordinated peers, whereas vertical SLAs deal with subordinated pairs within the service provision architecture stack. Horizontal SLAs are contracted between different parties providing the same kind of service. Vertical SLAs regulate the support parties get from their underlying infrastructure. Horizontal SLAs are defined at the same OSI layer (e.g., two IP domains). Vertical SLA are defined at different OSI layers (e.g., between the core MPLS network and an optical network) [34].

In general, the components of an Internet service can be classified into three layers: the application, the system, and the network. A set of SLA parameters at each layer can be included in an SLA. Application level parameters can provide the security, access, configuration, current status, and resource utilization of the Internet service entity and dependent components. System level parameters provide system health information that can affect the performance and reliability of Internet services. Network Level parameters are dependent on various transport technologies such as ATM, MPLS, Diffserv, etc.

An SLA contract between two parties usually contains technical and non-technical information further explored in the following subsections.

\subsubsection{Non-Technical Part}

There is tremendous effort to define SLA components in various research papers. The following lists some of the non-technical definitions.

- Customer and Service Provider Responsibilities: This part defines who is responsible for maintaining the hardware and software of the customer premise equipment. 
- Violation Procedures: This part defines procedures to be invoked in case of violation of SLS guarantees (e.g., mail, call).

- Service Pricing: This part defines the method of pricing, and discounting policies to apply when Service Provider commitments are not satisfied. Examples of pricing methods include one monthly fee, per trunk pricing (e.g., per MPLS LSP), and per packet/byte.

- Service Reporting: This part defines the method of reporting service qualities delivered to the customer. An example would be a periodic detailed report.

- Parameter change ability: This part defines the ability for a customer to change some of the SLA parameters dynamically while using the services.

- Service Provisioning: This part deals with the type of provisioned service. For example, a customer will be provided with redundant DS-3 connections to its web servers [28].

- Customer Support: This includes the typical helpdesk problem reporting and resolution guarantees. Examples include a single point of contact assigned to the customer and problem resolution within 48 hours of reporting [28].

\subsubsection{Technical Part - SLS}

While the non-technical part of SLAs is a human readable contract, it does not possess the technical sufficiency to achieve the end-to-end QoS control. The technical part of an SLA, consisting of technical parameters including QoS metrics (e.g., latency, throughput), availability, service schedule and their associated semantics, is called the Service Level Specification (SLS). SLSs can help fulfill the technical requirements imposed for achieving the end-to-end QoS control. 
Some metrics used in SLSs take the form of quantitative or qualitative attributes. A parameter is said to be quantitative whenever its value is expressed as a numeric value (e.g., 3 milliseconds), otherwise it is qualitative (e.g., "low", medium", etc.).

The following is a list of some technical parameters proposed to be used or in use in SLSs. It is not meant to be an exhaustive listing of parameters in an SLS but rather gives an overview of what to expect in the technical part:

- Service Schedule: This attribute reflects the "working hours" of the specified service. It can take the form of time of the day range (e.g., from 9am to 5pm), day of the week range, month of the year range, etc, $[25,50]$.

- Latency or Packet Transfer Delay: This metric is defined as the time difference between the time the first bit of a packet passing the ingress measurement point, and the time the last bit passes the egress measurement point at the other end. For example, for a Voice over IP connection to be usable, the one-way delay should be below $150 \mathrm{~ms}$ [41].

- Jitter: The variation in packet transfer delay is defined to be the variation in latency experienced by individual packets in a sequence of transmitted packets [41].

- Throughput: This metric defines the rate at which data is delivered to the customer (packets or bytes per second). For example, Intranet users will be able to load a $65 \mathrm{~kb}$ GIF file in under 10s during working hours [28]. Another example is that of a video stream defined at $1 \mathrm{Mbit}$ per second. At 0.9 Mbit per second, the stream cannot be displayed.

- Packet Loss Ratio: This metric is defined as the proportion of all sent packets of a population of interest that is received too late, or not received at all at the destination 
[41]. For example, compressed video can be very error-sensitive. A single loss of a delta frame encoding will affect the quality of the video stream for a long period of time.

- Flow Identification: This attribute refers to the flow defined by a set of datagrams that share some characteristics such as $\{$ Diffserv information, source information, destination information, application information $\}[25,50]$.

- Traffic Conformance: This attribute is a set of indicators that aim at describing how an IP flow should "look like" so that the customer would be serviced according to the level of quality described in the SLS. It takes the form of a peak rate, a token bucket rate, a bucket depth, a minimum packet size, etc. Usually there are two levels of conformance: either conformant (i.e., the traffic conforms to a certain leaky bucket algorithm) or non-conformant. Non-conformant traffic can be re-marked, shaped, or dropped $[25,50]$.

- Availability: The IP service is defined to be available at a given time if the Packet Loss Ratio is below a defined threshold t. For example, within a period of 5 minutes the Packet Loss Ratio is computed and checked if it is less than $99.9 \%$.

- Response time: This metric defines the maximum response time a service is permitted when handling user requests. For example, 95 percent of users will experience a response time of $2 \mathrm{~s}$ or less during work hours, where work hours are between 9 a.m. and 5 p.m. [28].

- Utilization: This metric defines the maximum service utilization allowed at which a service will perform within guaranteed response times and throughput. An example of 
this metric is that the system will support 32 simultaneous users during peak hours [28].

- Scope: This attribute, defined by a pair of ingress and egress interfaces, indicates where the QoS policy for the corresponding IP service offering is to be enforced [25, $50]$.

- Reliability: Reliability metrics consist of availability guarantees over a period of time. For example, the web server will be available 99.999 percent of the time it is accessed over a one year period [28].

- Excess Treatment: This attribute describes how out-of-profile traffic will be treated (i.e., dropped, re-marked, shaped, etc.) $[25,50]$.

- Spurious Packet Rate: Spurious packets are packets that are received at a destination, that have a valid IP packet header but were not transmitted at the sender that is indicated in the packet header [41].

\subsection{SLA Life cycle}

Several phases in the existence of a SLA have been identified $[1,27,41]$. Such partitioning will help in understanding the structuring of the research areas defined in the next section. The identified phases are summarized below:

- Product/Service development: This stage consists of the identification of the customer needs and the network capacities, from which service templates are prepared. Actually, a customer might be looking for certain service requirements that can fit into or would be accommodated by a service offered by a provider. 
- Negotiation and Sales: This stage consists of the SLA negotiation between customer and provider. It invokes the establishment of the agreement (legal binding) reflecting that the customer has actually subscribed to the service, is aware of the detailed, legally binding extent of what is comprised in the service delivery, etc. All required service subsystems, including access authorization, entries into billing systems, etc. need to be configured to accommodate this new service subscription.

- Provisioning stage: This stage consists of the resource provisioning/reservation and the service activation.

- Assurance Stage: This stage includes the performance related data reviewing by the customer (monitoring and validating the SLA), detecting and handling SLA violations.

- Assessment stage: This stage is composed of two parts: assessment with the customer to check the SLA satisfaction and possible re-negotiation of the SLA and internal operator assessment to check the overall service quality.

- Removal Phase: Associated configuration information is removed (Resources, billing entries, etc.). 


\section{Chapter 3}

\section{SLA Research Areas}

Several research projects and groups are actively working on SLAs or areas involving SLAs. The main Internet Engineering Task Force (IETF) groups working on this subject are:

- Internet Traffic Engineering (TEWG) [3]

- Real-time Traffic Flow Measurement (RTFM) [4]

- IP Performance Metrics (IPPM) [5]

- Remote Network Monitoring (RMONMIB) [6]

The Third Generation Partnership Project (3GPP) [7] which provides technical reports and specifications for a $3^{\text {rd }}$ generation Mobile System based on evolved GSM core Networks and the radio access technologies is another forum that has focused on SLAs.

Traffic Engineering for Quality of Service in the Internet at Large (TEQUILA) [8] is a European research project with primary goal to develop an integrated architecture and associated techniques for providing end-to-end QoS in a DiffServ-based IP network $[9,10]$. The TEQUILA project has taken the initiative in the IETF to propose a standard template for the IP related parameters and semantics of an SLS [11]. 
The Adaptive Resource Control for QoS Using an IP based Layered Architecture (AQUILA) [12] is another European research project defining, evaluating, and implementing an enhanced architecture for QoS in the Internet. There is a set of commonalities between the AQUILA and TEQUILA approaches. The main difference is that the AQUILA consortium has introduced the concept of predefined SLS types (Premium CBR, Premium VBR, Premium Multimedia, Premium Mission Critical) that are based on a generic SLS definition [15].

The Creation and Deployment of End-User Services in Premium IP Networks (CADENUS) [16] project is proposing an integrated solution for the creation, configuration and provisioning of end-user services with QoS guarantees in Premium IP networks.

The TeleManagement Forum (TM) [13] provides a performance reporting concept and SLA management handbook [14]. The objective of the handbook is to assist two parties in developing an SLA with a practical view of fundamental issues.

The following subsections divide the SLA research into 4 areas consisting of SLA parameter definition, SLA Management, SLA Monitoring and Reporting and SLA QoS Controls, collecting and partitioning all literature surveyed on the SLA subject.

\subsection{Parameter Definition}

Standardization efforts concerning Service Level Specification parameters try to address two main concerns: providing a standard set of information to be negotiated (statically or dynamically) between a customer and a service provider, and providing the corresponding semantics of such information so that it might be appropriately modeled and processed by either the customer or the provider. These efforts concern the definition of 
service level parameters such as availability, reliability, latency, loss, etc. as described in section 2.1.2.

In the following subsections, some standardized metrics are listed, some new suggested metrics are described, attempts to define languages and templates are described, and finally some research concerning the definition of new value-added SLAs is presented.

\subsubsection{Standard Metrics/Parameters}

Section 2.1.2 listed some SLS definitions that are found almost in every SLA. The problem of having non-standard SLS parameters is that each vendor will provide his/her own definition of the metrics, confusing the customer since each parameter is subject to interpretation, and making interoperability between service providers very difficult.

The IP Performance Working group [5] of the IETF has defined a framework for the specification of IP performance metrics [29] and is working on the identification of Internet service metrics. The Working group has so far defined two metrics dealing with the delay in IP networks: a One-Way Delay Metric for IPPM [31] and a Round Trip Delay Metric for IPPM [33]. The group has also defined two metrics that deal with loss and connectivity: a One-Way Packet Loss Metric for IPPM [32] and an IPPM Metrics for Measuring Connectivity [30].

\subsubsection{New Metrics/Parameters}

Some new metrics are also being proposed in recent papers. [2] introduces a weboriented, delay measuring performance metric "The Web Response Time Metric (WRT)". The WRT metric is defined as the amount of time from when a client issues a web-server request to when the entire web object has been successfully received by the client. This 
application-level performance metric offers several advantages over traditional ping-based metrics: the WRT metric seamlessly incorporates the impact of loss and latency dynamics on the application into the performance assessment; it is much more natural to translate WRT metric results into an assessment of how the network is impacting end users compared to an assessment based on loss or latency metrics. The paper speculates that it is easier for a service provider to guarantee an SLA based on the WRT metric than on latency and loss metrics, and thus the WRT can be the basis for future IP SLAs.

[40] advocates the need for a new metric to characterize IP service availability in the presence of link failures that represents the fraction of time the network is able to deliver IP packets to destinations. Link failures impact IP networks in several ways: rerouted traffic may congest links along the backup paths leading to higher loss, longer delays, and greater jitter, alternate paths setup may lead to black holes or transient loops, and routing convergence may take longer times. However not all link failures affect path availability (connectivity between a source and a destination). In fact, if there are several link-disjoint paths between two points, then path availability is not affected by multiple failures on a subset of these paths. The new metric, referred to as service availability, is defined as the ability of the network to deliver packets from the source to the destination. Service availability is different than port availability offered by ISPs representing the uptime of a single network element (the hardware by which the customer attaches to the ISP's network). The paper also claims that service availability definition must consider network topology, mapping of IP links onto the underlying physical infrastructure, inter-dependence of IP network elements, failure characteristics of links/routers and routing protocol convergence time. 


\subsubsection{Languages and Templates}

One of the main challenges for contracting SLAs is the chaotic definition of the parameters and what an SLA represents. Although an SLA is defined as a formal negotiated agreement, in the real world problems are still besieging clients and Service Providers. First, since commonly recognized references are not available, Service Providers have to establish SLAs from scratch, potentially creating more workload and dramatically slowing down the development of SLAs. Second, different Service Providers contract SLAs using a variety of perplexing jargons frequently driving customers into confusion and causing misunderstanding among providers. Third, deficient contracts with missing SLA parameters are not unusual leading to reputation impairment, economic loss, or even lawsuits [27].

[27] proposed using a "SLA Foundation Template Library with reusable-component repository" providing a comprehensive set of well-defined foundation templates for developing SLAs rapidly with quality. A SLA template defines a particular grade of service which can be offered with a SLA. The functionality of the template is to capture a set of service level objectives for a service along with details of consequences for not meeting the specified objectives, and potentially any exclusion conditions. The benefits claimed from such a library is the rapid development of SLAs, an easier negotiation between Service Providers and clients, a minimization of misunderstanding and confusion about the SLA terms used, a reduction in chance of missing parameters and a better SLA integrity, and partial automation by using computer aided design technology. The paper also suggests using the Unified Modeling Language (UML) as a legible graphical representation of the suggested objected-oriented extensible and flexible templates. 
[34] defines a language based on XML schema for defining SLAs: "SLAng". The advantages of using XML include the availability of tools such as "ECLIPSE" to edit and validate SLAs against the language specification and the ease of translation of SLAs into other representations using "XSLT" style sheets (e.g. more readable format). SLAng provides a format for the negotiation of QoS properties, the means to capture these properties unambiguously for inclusion in contractual agreements, and a language appropriate as input for automated reasoning systems or QoS-aware adaptive middleware. The paper argues that the main requirements of a language defining SLAs consist of: Parameterization, Compositionality, Validation, Monitoring, and Enforcement.

[50] describes a template for SLS defining attributes that can be dynamically negotiated between a customer and a service provider. The attributes are: SLS scope, flow identification, performance attribute (e.g. delay, loss, etc.), traffic conformance attribute, excess treatment attribute, service schedule, and reliability. The document aims at listing a set of basic parameters that will compose the elementary contents of an SLS that should facilitate the enforcement and negotiation of desired QoS services. It also describes example services, like a virtual leased line and funnel service, which can be built quickly using the defined parameters.

[51] describes a similar SLS template as the one defined in [50] involving an SLS type (e.g. Premium CBR), a scope, a flow identification, a traffic description, a performance guarantee and a service schedule). The paper also describes four suggested services based on the proposed SLS template described: Premium CBR, Premium VBR, Premium Multimedia, and Premium Mission critical. 
Yet another similar template is proposed in [53] which provides for a vendorindependent lexicon and extensible structures. The information contained in the proposed SLS template is structured into four categories: a Common unit containing customer, provider and service instance descriptors and validity sub-unit, a Topology unit containing a list of end-points and their inter-relationship, a QoS unit that defines the traffic descriptors, the load descriptors and the QoS parameters, and a Monitoring unit defining the scope and the reporting parameters. The paper also gives an example service, a Virtual Leased Line targeted for applications that require predictable point-to-point performance, which can be defined using the proposed SLS template.

\subsubsection{Proposing new SLAs}

As the complexity of the network features and ability to deliver various services increases, new SLAs are defined to be able to sell those features. Furthermore, Service Providers are looking for "attractive" SLAs as an incentive for customers to purchase their service versus a competitor's. [17] proposes a SLA based framework for QoS provisioning and dynamic allocation. The proposed SLA allows for dynamic capacity allocation based on instantaneous demand. The "Three Tier Pricing model with Penalties" (TTPP) SLA gives incentives to the users to relinquish unused capacities and acquire more capacity as needed. The customer buys a long term capacity at a pre-specified price and is given a discount if he relinquishes a portion of that capacity. On the other hand the customer is charged a higher price if he demands an increase in capacity. The paper also discusses a scheme to solve the admission control problem arising from the TTPP SLA using the concept of trunk reservation. 
[24] proposes a range-based SLA where customers specify their requirements as a range of quantitative values rather than a single one. For example, if the user is not sure on whether he needs $0.5,0.6$ or 1 Mbps then he can specify a range on the bandwidth (e.g. [0.5, 1]). With range-based SLAs only the lower bound capacity needs to be guaranteed and the multiplexing effect in the core leaves enough room to adopt a more aggressive approach and actually accommodate more connections that it is possible with regular non-range based SLAs. It was shown that with range-based SLAs, providers have the flexibility to allocate bandwidth that falls within a low and high segment and take advantage to make multiplexing gains in the core that are not possible with a deterministic approach. Provisioning such SLAs required frequent changes or re-configurations of the interior nodes, the solution of which involved capacity inventory of the interior nodes to be updated based on VPN connection acceptance at the edge.

\subsection{Management}

The second research area concerning SLAs is SLA Management, which deals with how to manage and control the QoS delivered to customers to ensure compliance with established SLAs. An SLA has to be managed efficiently to be valuable. SLA management will enforce the confidence customers have on their use, and support transition from traditional usage of services, to more elaborated usage of these new services.

A well known aspect of management is the Management Information Bases (MIBs). Several (MIBs) are related to the performance management of Internet Services. These include the Host Resources MIB, Network Services Monitoring MIB, Application MIB, and SLA performance monitoring MIB [28]. 
[56] lists open issues on SLA management focused on three particular points:

- The information model of SLS (contents of the SLS): The SLS template is not clearly defined. It is mandatory for the SLS to be defined by a template or an information model to allow cooperation/negotiation between entities.

- The SLS negotiation protocol: divided into three aspects: functional, security and inter-domain.

- The end-to-end point of view: crossing multiple domains.

Interconnections among IP networks are established by means of SLAs which require high manual overhead and a high associated cost which limits their wide-spread use. This causes scarce providers' revenues and a non-optimized resource allocation of resources inside the network. Such agreements can not be managed statically and on a long-term timescale automatic SLA management has to be addressed. [64] introduces the notion of Elastic network node both between each pair of network providers and between corporate networks and access network providers, in order to operate some statistical evaluations on traffic flowing among them in order to produce an automatic request of the most appropriate SLAs among various network operators. The introduction of automatic mechanisms for SLA management can lead to a cost reduction and enable the creation of short-term services.

[28] provides a utility model, where utility is defined as the satisfaction obtained from a service provider of the consumed system and network resources, to capture the management and control aspects of SLAs for multimedia Internet service. The model is based on the concepts of quality profile, quality-to-resource mapping, resource constraints, 
and utility function. It provides a computationally feasible solution for admission control and quality adaptation for multimedia Internet service for SLA conformance.

In addition to the access link SLA, a major challenge is the end-to-end SLA management, which induces the propagation of SLA requirements across technologies and between different provider networks. End-to-end SLA management implies supporting multi vendor, multi domain, multi technologies and negotiation capabilities. To reduce service deployment time, it is necessary to introduce a standard for negotiation including templates (such as the ones mentioned in section 3.1.3) and protocols between providers that will simplify the propagation of SLAs across the network [1].

[56] also lists challenges in the fulfillment part of the SLA management which includes Resource Admission control, Allocation management and Resource Allocation Request Handling. And finally, it lists the main issues of assurance in SLA management:

- QoS metrics computation and QoS metrics report management (many standardization issues)

- Performance management (data collection and network measurement)

- Problem management (automatic handling, root cause analysis)

- Forecast function (recommendation for performance improvement)

Finally, [67] proposes a SLA management system for IP domains based on use of the Mamdani type of fuzzy controller that takes as input a customer satisfaction ratio and a provider profit ratio and outputs a variable that indicates how suitable the state of the controlled SLA from the ISPs point of view. The well-known advantage of the Mamdani controller is that system experts (network operators) can affect control operations of controllers easily by tuning the controllers according to their heuristical control aims. The 
customers are allowed to send information about the satisfaction of the offered service; however the ISPs decide how the opinions of the customers are considered in the control operations. Weighting of the satisfaction levels of the customers enables the ISPs even to consider the needs of the individual customers differently during the control operations. Simulation results indicate that the management system was capable of controlling both the economic profit of the ISPs and satisfaction of the customers, although the operation aims of the ISPs varied heavily.

The Admission control and the Accounting aspect of management are addressed in the following sections.

\subsubsection{AAA}

The Authentication, Authorization and Accounting (AAA) Working Group [42] focused on the development of requirements for Authentication, Authorization and Accounting as applied to network access.

Generating billing and credit records as per SLAs have not yet reached a high degree of automation [55]. However, the description of the accounting method in an SLA is an important component. In general there are three main models of charging (a mix of the models can also be found) [41]:

1. Flat rate accounting, where a user pays a periodic fee for the service regardless of the usage. It is the simplest of the models and does not require any measurements or data gathering for charging purposes.

2. Reservation based accounting, where a customer can select different QoS levels (can be dynamic reservation) and pays a different price for each level within the period reserved. This charging scheme is more complex than the flat rate scheme in 
the sense that data has to be gathered about customers changing the QoS level of their service. These changes however are expected to occur at relatively large time scales.

3. Actual usage based accounting, where the actual use of the service is measured at very fined grained level (short time periods). The numbers of sent/received bytes in each QoS class has to be gathered and charging is proportional to those numbers. Obviously this model requires the most complex data gathering mechanisms. [41] claims that flat rate charging schemes tend to increase the overall usage of the service, whereas a usage based charging scheme acts as an incentive to make little use of the service.

Much effort and many resources are directed to pricing schemes research [61]. Among them are the Flat-Price model, Paris-Metro Pricing, Smart-Market Pricing, Edge Pricing, Priority Pricing, Expected Capacity Pricing, and Responsive Pricing. [61] presents a new per-packet pricing scheme in which the payment is collected on a per packet basis in each router on the packet path. The scheme is analogous to a toll-road where each router is at the entrance to the next toll-road on the path of the packet. Each packet carries "electronic money" (User identity field, Accumulated charge field, and Money limit field) that is used to pay routers in return for services. A central database polls the routers for information about accumulated charge field per user identity. The pricing scheme takes into account also the congestion times so that during a higher congestion period the per-packet toll price is increased, whereas in a low congestion period the toll charge is decreased. The paper suggests that the new pricing scheme increases both service provider revenue and the user's welfare, since service providers increase their revenue when the network is congested, and users' provided services are not penalized when other users are consuming the bandwidth. 
For Mobile environments, [59] proposes a concept to combine the Service Location Protocol and the Mobile IP AAA based architecture. This allows the support of mobile telephony over packet based IP networks without requiring GSM-like mobility management and accounting schemes.

\subsubsection{CAC}

Connection Admission Control (CAC) is the component that takes a decision on whether to accept or reject a request for service based on resource availability. There is a close relation with pricing and managing connections in ATM networks and IP networks. The main difference is that SLAs for differentiated services are of a more static nature and the level of performance guarantees can be loose. Hence admission control is less strict, and has the goal of ensuring an average level of performance.

[21] presents an approach to manage and price SLAs for DiffServ $[9,10]$ that uses a simple upper bound for the effective bandwidth of the conforming traffic as a proxy for resource usage. The bound considers an on-off approximation of the input traffic with a peak rate which depends on the traffic contract parameters (peak rate and token bucket parameters), while keeping the same mean rate. Usage charges for specific time periods are proportional to this approximation, and their calculation requires only measurements of volume.

[22] attempts to solve the bandwidth provisioning problem for a service overlay network, which purchases bandwidth with certain QoS guarantees from individual network domains via bilateral SLAs to build logical end-to-end service delivery. The solution takes into account the SLA, service QoS, traffic demand distributions, and bandwidth costs. Analytical models and approximate solutions for both static and dynamic bandwidth 
provisioning are developed. It was found that for static bandwidth provisioning it is more beneficial to overprovision more bandwidth; however, it is inefficient in terms of resource usage if traffic demands are highly variable. In such kind of environments, the dynamic bandwidth provisioning model is better, albeit with more expensive network resource management.

[23] studies path provisioning as a mechanism to deliver SLAs in IP differentiated services networks. It considers static SLAs, more likely to be used in ISP's core networks where multiple traffic flows produce less dynamic aggregated behavior, since any significant change in the SLA causes re-computation of the provisioned paths. The path provisioning problem is partitioned into smaller problems, each handling a Diffserv class starting from EF $[9,10]$. First, the provisioned paths for the EF traffic are computed and pinned down, and the amount of available bandwidth on the links is correspondingly adjusted. The same procedure is repeated for the other DiffServ traffic classes. The reference compares several algorithms that compute routes for the provisioned paths either centrally by a QoS manager or in a distributed manner by the source nodes.

[57] proposes a structure for QoS-centered SLAs and a framework for their real time management. The proposed SLAs are for QoS assured delivery of aggregate bandwidth, however the control and signaling are for the more granular flows. The basic idea behind the SLA is that if the Customer is requesting bandwidth not in excess of the contracted amount then the Provider is expected to carry bandwidth that is in the same proportion to what is being requested. On the other hand, if the Customer is requesting stream bandwidth which is in excess of the contracted amount then the Provider is only obligated to carry the carried bandwidth. A monitoring scheme is also introduced in which revenue is generated as flows 
are accepted into the network and penalty is incurred when flows are lost. The loading status of routes is pre-calculated off-line and measurements are taken locally at ingress nodes in the proposed management scheme. A simulation test-bed was used to verify that the proposed SLA management scheme is robust, fair and efficient over a broad range of traffic conditions. [60] defines an analytical approach and a methodology to determine the set of SLAs that can be effectively supported by a DiffServ IP network. Based on the SLA probabilistic description, the paper derives a worst-case mathematical formulation for the overbooking probability. The implementation of the proposed admissibility decision requires the global knowledge about the current active SLAs and the paths of the corresponding traffic i.e. it requires a centralized QoS server in the network.

\subsubsection{Negotiation}

Negotiation is the second aspect of SLA management besides CAC and AAA. To date, most of the service level negotiation process has been carried out manually where the typical assumed timescale for SLA modification is one month or more (more or less static SLAs). Frequent modification is almost impossible due, for example, to the potential interactions between SLAs and the constraints at the enforcement points. When modification is needed, the customer may require the assistance of the network provider. The provider, in turn, may need to reengineer part of a system to ensure the mandated service levels.

[35] defines a dynamic SLA as a contract between client and provider in which a subset of parameters can be changed over time. In order for the service level negotiation to be dynamic on a large scale, SLS negotiation protocol is almost mandatory. Since protocols for end-to-end QoS service realization and negotiation, like COPS [36], have been proposed for the Internet and vendors such as Cisco Systems, Juniper Networks, Extreme Networks 
and Nortel are already providing equipment running COPS [55], the paper proposes an extension of the COPS protocol for intra and inter-domain dynamic SLS negotiation. The proposed protocol, named COPS-SLS, allows for configuring domain policies regarding service levels and negotiating automatically of service levels within the domain policies. COPS-SLS [54] uses a Policy Information Base (PIB) to represent SLS information enabling a high level of independence and reusability. In addition, the organization of the SLS negotiation process in two phases (configuration and negotiation) allows the automatic configuration of the negotiation process. There are three main motivations for using COPS for SLS negotiation: policy-based networking as network management, flexibility of the COPS protocol to convey policy information, and keeping the network management homogeneous by use of COPS in both negotiation and service deployment.

The re-negotiation of an SLA can be triggered by several events linked to critical problems such as the efficiency of network resource allocation, the performance of user's application, and the reduction of communication costs. Results provided by traffic measurement (monitoring) represent an important element on the basis of which users might request an SLA re-negotiation. Monitoring and reporting an SLA is described in the next section.

[50] lists some requirements for an SLS negotiation protocol: It should use a reliable transport, it should provide for a dynamic SLS negotiation and subscription procedure, it should support a mechanism that may be used for statistical information retrieval, and it should support the appropriate security mechanisms to provide some guarantees as far as confidentiality. 


\subsection{Monitoring and Reporting}

The third research area involves monitoring and reporting. Monitoring deals with how to accurately measure the contracted service (such as the QoS parameters) that service providers deliver to their customers. Valuable network and application performance statistics can serve as the basis for effective SLA management. On the other hand, reporting deals with mechanisms to satisfy increasingly sophisticated customers who demand real-time reporting to confirm that they are receiving the service levels they were promised.

QoS Networks provide users with a portfolio of services negotiated through SLAs. The subscription to such services requires auditing; from the customer's perspective, monitoring will be required to verify whether or not the provider actually meets the conditions specified in the SLA. In fact, both the provider and the customer will want to verify if the SLA is being met. The Provider wants the gathered data from the service infrastructure to make a bill and detect any failures in his network; the customer wants the data to be able to verify the accuracy of the bill and verify that the paid for service is being provided.

QoS monitoring is required to track the ongoing QoS, compare the monitored QoS against the expected performance, detect possible QoS degradation, and then tune network resources accordingly (maybe even re-negotiate SLAs as described in the previous section). [28] classifies measurement mechanisms into passive probe, active probe, polling MIB and classical tools and describes the characteristics of each.

[58] provides a scalable monitoring approach for SLA validation. Since the information exchanged between network nodes and a central entity that reports the collected measurements or uses them to compute network refinements could be substantial, the paper 
suggests a monitoring algorithm based on selective hop by hop measurements. The measurements are aggregated into histograms, well-suited to approximate curves, which can be selectively refined. Either a network manager starts polling all agents or agents periodically send their initial histograms. The approach was verified through simulations and proven to be at least an order of magnitude more efficient than a simple polling scheme.

[68] describes a non-intrusive scheme to measure end-to-end latency based on the knowledge of the queuing behavior. The conventional approach to measure the performance metrics is on an intrusive basis that may cause extra burden to the networks. Regardless of whether a one-way or two-way delay measurement, there is a drawback such that the accuracy of measurement greatly depends on the sampling method. On one hand, insufficient testing packets make the measurement results unreliable, and on the other, too many testing packets may cause extra burden on the network and change its actual load. The proposed scheme is non-intrusive since it is based on queue length monitoring. After each queue length measurement period on every router along the end-to-end path, the time-stamped measurement is forwarded to a central management unit that can reconstruct the queue state distribution at each stage and estimate the end-to-end delay based on the expected queuing delay and the fixed propagation delay on each link.

Finally, [39] defines a set of new documents named Service Level Indication (SLI) aiming at indicating whether measured data, related to a specific service instance, are in accordance with the QoS level specified in the SLA. Three kinds of SLIs are identified:

- Template SLI: provides a template for the creation of documents containing the monitoring data associated to a specific service. 
- Technical SLI: contains detailed information about the resource utilization and/or a technical report based on requirements stated in the SLS (based on XML).

- User SLI: contains information about the service conformance to the negotiated SLA (kept in a friendly fashion).

The SLI model is based on the CADENUS [16] architecture which defined three functional components acting as mediators between a user and the resources involved in the service provisioning: Resource, Service and Access Mediators. In order to allow users, service providers and network operators to have information about QoS parameters and network performance the need arises to export data collected by measuring devices (mediators). Such reports can be obtained in push mode, pull mode or periodic intervals. The following two sections describe tools, techniques and research projects that deal with the monitoring and reporting aspect of an SLA.

\subsubsection{Tools and Techniques}

This section lists some of the available measurement tools and techniques that can be used to actually carry out measurements that are needed for verification.

- Netramet: This is an implementation of the Meter MIB [43]. The basic operation of the system involves the creation of rule-sets defining criteria that determine which packet belongs to which flow. The rule-sets are then downloaded into the Netramet meter which processes matching packets against the installed rule-sets and counts packets within each individual flow [41].

- Ntop: This is an integrated tool that can do various kinds of measurements and types of analysis on IP traffic. The four main functions of Ntop are traffic measurement, traffic monitoring, network optimization and planning, and detection of network 
security violations. Ntop is available as an open source package [44]. The tool can capture and analyze traffic separately from various hosts in a broadcast environment [41].

- MIB-II: The IETF defined the MIB-II [45] as a MIB that all compliant devices should implement and in fact has been deployed by the majority of IP capable equipment. Many IP related metrics can be derived by measurements taken from MIB-II.

- Remote Monitoring (RMON): This standard is defined by IETF and enables the monitoring of complete network segments consisting of multiple hosts. RMON currently exists in two versions (RMON-1, and RMON-2) which are described in a set of four documents [46, 47, 48, 49]. RMON-1 can only be used to monitor networks at the Ethernet layer, whereas RMON-2 adds capabilities to RMON to also monitor layers 3 up to 7 of the OSI model. RMON can be used to determine spurious packet rates, error ratios and loss ratios within a single broadcast domain along with throughput verification but it cannot measure transfer delay or variations in transfer delays [41].

- Multi Router Traffic Grapher (MRTG): This tool can monitor sent and received traffic along with occurred errors at router interfaces, and present the results in a graphical form on a web page. The information that MRTG provides can be used as an indication for the values of the throughput and error ratio [41].

\subsubsection{Research Projects}

[28] lists some research projects related to SLA measurements, repeated in Table 2 for convenience. 
Table 2 Research Projects related to SLA measurement

\begin{tabular}{|l|l|l|}
\hline Project & Description & Organization \\
\hline Skitter & $\begin{array}{l}\text { Measures forward IP paths (each hop) from a source } \\
\text { to many destinations }\end{array}$ & DARPA/NSF/CAIDA \\
\hline AMP & $\begin{array}{l}\text { Intended to improve the understanding of how high } \\
\text { performance networks perform and to help in } \\
\text { problem diagnosis for both the network's users and } \\
\text { its providers }\end{array}$ & NSF/NLANR/Internet 2 \\
\hline NIMI & $\begin{array}{l}\text { Measures the global Internet; NIMI probes can be } \\
\text { delegated to administration managers for } \\
\text { configuration information and measurement } \\
\text { coordination active monitoring of end-to-end }\end{array}$ & DORPA/NSF \\
\hline PingER & $\begin{array}{l}\text { Provides actenet/HENP/XIWT } \\
\text { performance of Internet links }\end{array}$ \\
\hline Surveyor & $\begin{array}{l}\text { Provides active tests of one-way delay and packet } \\
\text { loss along paths between measurement machines }\end{array}$ & CSG/Advanced \\
\hline $\begin{array}{l}\text { RIPE } \\
\text { Traffic }\end{array}$ & $\begin{array}{l}\text { Provides independent measurements of connectivity } \\
\text { parameters such as delays and routing vectors }\end{array}$ & RIPE \\
\hline IPMA & $\begin{array}{l}\text { Studies the performance of networks and networking } \\
\text { protocols in local and wide area networks; also } \\
\text { collects routing and network statistics }\end{array}$ & Merit \\
\hline
\end{tabular}

\subsection{QoS Controls}

The fourth research area encompasses the mapping of the Service Level Specification (the technical parameters such as a delay bound, a guaranteed throughput, etc.) described in an SLA to QoS controls, mechanisms or components (such as schedulers, queues, policers, etc.) needed in providing the specification defined in such SLA.

An example of such QoS mapping is found in [62]. The paper proposes a hierarchical multilayer QoS routing system with dynamic SLA management for large-scale IP networks, by augmenting the existing OSPF and CR-LDP protocols to support hierarchical QoS routing, QoS aggregation, and QoS reservation in a MPLS-DiffServ based hierarchical routing network. A policy server is also proposed that is capable of dynamically handling SLAs between the networks and providing load balancing management within the network. 
The proposed hierarchical QoS routing reduces the MPLS LSP blocking probability and significantly reduces the control overhead.

Another example could be found in $[65,66]$. The papers present a framework for dynamic management of SLAs in MPLS networks. In the proposed framework, the ingress router of the MPLS backbone performs admission control as well as path selection. For path computation different advanced routing algorithms are used. In the paper, the MPLS SLA is mapped to finding an MPLS LSP that can support the SLA by finding an appropriate path for the LSP. Three routing algorithms were inspected, Lowest-Cost (LC) routing algorithm, Widest Bandwidth (WB) routing algorithm and Minimum Interference Routing Algorithm (MIRA), where performance was judged based on average end-to-end delay, average throughput, average packet dropping probability, average link load and number of utilized links. The paper found through simulations that although LC and WB perform well when the number of requests is small, the MIRA algorithm proves to show more stable values and better performance of these parameters at high network loads. MIRA results in smaller average end-to-end delay and smaller average number of dropped packets whereas still utilizing a smaller number of links in the network.

Finally, [38] discusses a QoS mapping at the application level to label packets relatively to each other. The paper proposes a framework for QoS mapping between practically categorized packet video and relative DiffServ network employing unified priority index and adaptive packet forwarding mechanism. In this framework, for example, a video application at the source grades the chunks of its content by certain indices according to their relative importance (in terms of delay and loss) in end-to-end QoS. The indices (Relative Delay Priority Index and Relative Loss Priority Index) reflect the desired service preference 
of a packet compared to others. Then, the QoS control takes place in the form of assigning each packet with an appropriate DS level (called QoS mapping). 


\section{Chapter 4}

\section{SLAs and Network Technologies}

With the existence of several network technologies and providers offering connectivity to such technologies, several variations of SLAs have been devised within each network. The following sections give a brief overview on SLA definitions within each network technology.

\subsection{IP Networks}

Many corporate WAN architects are considering migrating from costly leased line circuits to "private IP" services. In order to do so, IP service providers must provide SLAs that offer robust assurances that cover service availability and performance (while IP SLAs in the form of availability, latency and loss are offered, they are generally confined to the backbone). The industry direction is to model IP SLAs after those offered by frame relay networks. The reality however is that the SLAs surrounding today's private IP services only loosely mirror frame relay SLAs. 
WorldCom/UUNET's VPN was one of the first provider solutions to offer a true endto-end SLA commitment. The commitment assures that the VPN service will be available $99.8 \%$ of the time averaged over 3-9 sites with latencies less than $120 \mathrm{~ms}$ between customer premise equipment routers within North America. Measurements, based on periodic pings, are averaged over one month at all eligible sites. An eligible site is defined as a site whose sustained usage level is less than or equal to $50 \%$ of the total dedicated capacity. If sustained use exceeds 50\% during two consecutive months, customers must order an upgrade within a 30 day period or the site becomes ineligible for the SLA. If an SLA violation occurs in two consecutive months, UUNET provides credit back to the customer [2]. It is observed that the assurance associated with the IP SLA is very weak compared to provided frame relay SLAs.

After Intserv, the Diffserv approach allowed different QoS grades to different classes of aggregated traffic flows. Diffserv Working Group in IETF has defined two services: a premium service (EF), which expects the virtual leased line service to support low loss and delay/jitter, and an assured service (AF), which provides better than best effort but without guarantee. Many research projects, like TEQUILA, AQUILA and CADENUS (described below), addressed the issue of how to build services over the proposed Diffserv approach. Examples of IP services proposed by TEQUILA [8] are IP Premium Service, Qualitative Olympic Services, bi-directional Virtual Leased Lines and Virtual Private Networks. A proposal was made in $[25,50]$ to standardize some of the SLS parameters and build services based on them. For example, a virtual leased line can have the following attributes: a certain scope (ingress, egress interfaces), a flow id (packets sourced from a certain IP address and destined to a certain IP address), some traffic conformance parameters 
(e.g. (b, r, drop) of a leaky bucket algorithm), some performance parameters (e.g. delay $=$ $20 \mathrm{~ms}$, loss $=0 \%$, etc.), a service schedule (e.g. 24/24, 7/7), and a certain reliability.

Not many operators are yet exploiting DiffServ to offer QoS guarantees to their customers, in spite of the potential increases in revenues. This fact is due in part to the difficulty in setting the parameters of the algorithms used for the differentiation of the treatment offered to the different traffic classes, but even more to the difficulty in determining how to implement an SLA Admission Control algorithm so as to protect the network from overloads, thus allowing the network to meet the QoS guarantees specified in the SLA contract [60].

In fact, so far, IP Service Providers are not able to guarantee any availability and loss rate parameters. These ISPs offered price is often based on the 95-5 model. In this model, the customer buys a committed bandwidth for a fixed price. The customer is allowed to send traffic at higher rates than the committed bandwidth and the provider measures the average bandwidth usage of each customer in every five minute period by measuring the total traffic sent or received by the customer in five minutes interval. These averages are then accumulated over a period and then sorted. The highest five percent of the samples are discarded to compute the $95^{\text {th }}$ percentile of these samples. The difference between the so computed $95^{\text {th }}$ percentile and the committed bandwidth is charged at a higher rate [17].

The following sections describe some research projects or consortiums of companies that tried to address some IP SLA definitions.

\subsubsection{TEQUILA}

The European project TEQUILA [9] (Traffic Engineering for Quality of Service in the Internet at Large Scale) main objective is to study, specify, implement, and validate 
service definition and traffic engineering tools for the Internet. It is aiming to provide both quantitative and qualitative guarantees through planning, dimensioning, and dynamic control of traffic management techniques based on DiffServ [9,10]. TEQUILA along with AQUILA and CADENUS (see below) deal with the deployment of value-added IP services over the Internet.

The project (TEQUILA) addresses the following technical areas:

a. Specification of static and dynamic intra and inter-domain SLSs

b. Protocols and mechanisms for negotiating, monitoring, and enforcing SLSs

c. Intra and inter-domain traffic engineering schemes to ensure that the network can cope with the contracted SLSs within domains and in the Internet at large. Members of the TEQUILA project introduced some IETF internet drafts $[25,26,50]$ on the SLS topic, that address service level specification semantics, parameters and negotiation requirements and a framework for SLS usage. The drafts are referenced in other sections of this thesis.

\subsubsection{AQUILA}

The Adaptive Resource Control for QoS Using an IP based Layered Architecture i.e. AQUILA [12] consortium aims at defining and implementing a layered architecture for the support of QoS in IP networks. In the AQUILA architecture, a reservation request is sent by the so called "End user application toolkit" to the "Admission Control Agent". The reservation request specifies the required resources and QoS level and provides the information needed to identify the flows to which the reservation applies. Resources are managed by the "Resource Control Agent". The main difference between the AQUILA and TEQUILA approaches is that the AQUILA consortium has introduced the concept of 
predefined SLS types that are based on the generic SLS definition. The AQUILA consortium had a contribution defining an SLS template as an IETF Internet Draft [51].

\subsubsection{CADENUS}

The CADENUS project [16] is proposing an integrated solution for the creation, configuration and provisioning of end-user services with QoS guarantees in Premium IP networks. CADENUS will provide service creation and configuration in a dynamic way through the appropriate linking of user related service components (authorization, registration, etc.) to network related service components (QoS control, accounting, etc.). For more information concerning project deliverables please refer to [16].

\subsection{ATM Networks}

ATM networks are a bit different than IP networks in that they are mostly found in backbones. A typical ATM SLA, such as the one currently offered by WilTel [52] can be summarized as follows:

Network availability: 99.999\%

Mean time to Restore:

Network Equipment: 2 hours

First Fiber Restore: 4 hours

Latency:

CBR: $\quad 50 \mathrm{~ms}$

VBRrt: $\quad 55 \mathrm{~ms}$

VBRnrt: $\quad 60 \mathrm{~ms}$

Packet Loss: 
CBR: $\quad$ 1.00E-09

VBR: $\quad$ 1.00E-06

Cell Delay Variation (CBR, VBRrt):

DS-3: $\quad 2$ millisec

OC-3: $\quad 500$ micro sec

If any metric is not upheld, the applicable credits are:

$1^{\text {st }}$ month an SLA is not met: $\quad 50 \%$ credit of the affected port and PVCs

$2^{\text {nd }}$ month an SLA is not met: $\quad 75 \%$ credit of the affected port and PVCs

$3^{\text {rd }}$ month an SLA is not met: $\quad 100 \%$ credit of the affected port and PVCs

\subsection{Frame Relay Networks}

The Frame Relay Forum (37) has defined three performance metrics that can be used as the basis for an SLA:

- Network availability: defined as the ratio of network up time divided by the total amount of time associated with the metric

- Network latency: defined as the maximum one way frame latency over the frame relay network (end-to-end between frame relay devices at the customer site).

- Data Delivery Ratio (DDR): defined as the percentage of data (i.e. bytes) that is successfully sent over the frame relay network (applicable only to traffic that does not exceed the Committed Information Rate (CIR)). A related metric, the Frame Delivery Ratio (FDR) is the percentage of frames sent successfully over the network.

As an example, Sprint's domestic frame relay service offers the following SLA [2]: 
- Availability: Sprint offers $100 \%$ availability when they provide a SONET access connection and $99.99 \%$ availability over Sprint-provided non-enhanced (not SONET) metropolitan-area access.

- Permanent Virtual Circuit (PVC) delay: Sprint's latency commitment depends on the access speeds and the class of service associated with the data. For T1 access, the maximum one-way end-to-end delay will be within 70 milliseconds throughout the continental United States.

- Loss: Sprint offers a 99.9\% FDR commitment for traffic within CIR.

In a Frame Relay Network an SLA typically includes QoS metrics such as committed bandwidth, transit delay, packet (or cell/frame) loss rate and availability. Most of the Frame Relay providers are converging at the same QoS parameters of 99.99 percent availability, 0.001 percent loss rate and 50-100 ms of one way transit delays. The pricing of these services is mainly based on the fixed committed bandwidth (Committed Information Rate - CIR) [17].

\subsection{MPLS Networks}

Until IP differential services are available, IP SLAs will be problematic for service providers due to the lack of robustness and guarantees in IP networks. With the help of MPLS, a service provider can confidently offer latency and loss assurances within their backbone network [2].

Traditional IP VPN technologies, such as IPsec, GRE and L2TP, are difficult to guarantee QoS and cannot provide effective service management solution because of the connectionless nature of the IP protocol. These problems can be solved by the emerging MPLS technology by which end-to-end VPN service can be realized and Traffic Engineering 
can provide customers with quantitative and qualitative QoS guarantees. The SLS for MPLS VPN can be classified into two types: "point to cloud" and "point to point". The former is supported by mapping to DiffServ traffic conditioning and PHBs. For the latter, more accurate quantitative QoS and more service types can be supported. Communication modes, traffic conformance, service performance and service schedule describe the SLS. Potential conformance parameters include: peak rate, token bucket rate, bucket depth, burst rate, minimum MTU and maximum MTU [63].

\subsection{Wireless Networks}

The "Third Generation Partnership Project" (3GPP) provides technical specifications and technical reports for a $3^{\text {rd }}$ generation mobile system based on evolved GSM core networks and the radio access technologies that they support. 3GPP has defined four traffic classes based on delay requirements listed in Table 3.

Table 3 3GPP QoS classes classification [56]

\begin{tabular}{|c|c|c|c|}
\hline \multicolumn{4}{|c|}{ Traffic classes } \\
\hline Conversational Class & Streaming Class & Interactive Class & Background \\
\hline Delay $<150 \mathrm{~ms}$ & Delay $<1 \mathrm{~s}$ & Delay $<1 \mathrm{~s}$ & Not guaranteed \\
\hline $\begin{array}{l}\text { Preserve time relation } \\
\text { (variation) between } \\
\text { information entities of } \\
\text { the stream (stringent } \\
\text { and low delay) }\end{array}$ & $\begin{array}{l}\text { Preserve time } \\
\text { relation (variation) } \\
\text { between information } \\
\text { entities of the stream }\end{array}$ & $\begin{array}{l}\text { Request Response } \\
\text { pattern. } \\
\text { Preserve Payload } \\
\text { content }\end{array}$ & $\begin{array}{l}\text { Destination is not } \\
\text { expecting the data } \\
\text { within a certain } \\
\text { time. Preserve } \\
\text { Payload content }\end{array}$ \\
\hline
\end{tabular}

$[18,19]$ describe a framework, based on the extension to the CADENUS framework [16], that can provide an effective, network independent, end-to-end QoS control. The framework describes how dynamic SLA-based control can be used to achieve end-to-end 
QoS in a wired and wireless (UMTS) environment. UMTS is a standard for third generation wireless networks that defines the four QoS traffic classes mentioned above i.e.:

- Conversational: provides strict delay guarantees, used for voice and video applications.

- Streaming: slightly relaxed in terms of delays, used for streaming applications.

- Interactive: follows a request response pattern and can justly provide qualitative guarantees.

- Background: no qualitative or quantitative guarantees, used for the "best effort" traffic consisting of bulk and asynchronous traffic (email, ftp).

[18] summarizes the UMTS QoS classes attributes, like the Maximum bit rate, guaranteed bit rate, allocation priority, etc. and describes the mapping of these classes to the Diffserv $[9,10]$ defined classes.

In the CADENUS [16] framework, service contract definitions are of two types: 1. retail SLAs as a unitary service between the customer and the service provider and 2 . wholesale SLAs for contracts between service providers. Those SLA contracts are signed (static) when the customer subscribes to service provision. From the perspective of wireless networks, since QoS availability varies considerably during service usage due to handovers, fading, etc. it should be possible to negotiate QoS on the fly necessitating the use of dynamic SLAs. SLAs can be negotiated per session (during session initialization using PDP) or in the midst of a session typically through re-negotiations $[18,19]$.

With the nature of the dynamic SLA negotiation in wireless networks, there were some suggestions to use a modified COPS protocol for that purpose. [20] introduces a dynamic SLA management procedure to be used in cellular Diffserv networks. Since 
performing CAC in wireless networks, involving SLA negotiation and QoS provisioning, is not adequate for the overall admission control of the handoff request due to time constraints, a virtual CAC method is proposed in order to support dynamic SLA management. Based on information received from a decomposed handoff messaging, target base station becomes capable of initiating the virtual connection admission control for the incoming mobile in very advance. As a result, much shorter handoff latency than the conventional methods is achieved. 


\section{Chapter 5}

\section{3-Tier SLA with Automatic Class Upgrades}

So far, we have seen various terms and definitions used in SLAs, highlighted four research areas, and displayed applications in IP and legacy networks. Concerning the definition of new SLAs targeted to attract new clients, telecom operators are reacting to their shrinking circuit switched networks with their own innovative voice solutions, often based on voice over IP (VoIP). They are suggesting new services or SLAs bundling classes of services and targeting voice, data, and video. Such new services or SLAs are viewed as being essential to revenue maintenance, customer retention, and growth. An example of such services is the "triple play" SLA which bundles 3 separate channels for 3 classes of services used by voice, video and data, respectively.

One enhancement suggested to the "triple play" SLA is the 3-tier SLA with automatic class upgrades. The SLA is specified as a bundle of 3 classes of service. The customer pays a fixed rate per service class, where the price of each is a function of the desired bandwidth for that class. The provider sets up his network to accommodate the 3 reserved rates and guarantees the reserved bandwidth to the customer. Remarking of packets is done at the 
provider's edge nodes to fill up the highest quality (and thus most expensive) reserved bandwidth classes first. From the customers' perspective, the SLA guarantees full usage (when there is enough traffic to fill it) of unused bandwidth in the most expensive classes, and thus puts customers' "money" to the most effective use. Providers, on the other hand, can sell the suggested SLA (with probably different pricing models) as an edge over competitors providing "triple play" like SLAs without automatic upgrades.

We describe in section 5.1 an analytical example of the 3-tier SLA, then suggest in section 5.2 some QoS controls and components that can provide the SLA. In section 5.3 we describe the reordering caveat due to class upgrades, and then propose a solution along with its proof in sections 5.4 and 5.5 , respectively.

\subsection{Description}

The 3-tier SLA with automatic upgrades can be summarized as shown in Table 4. It is specified in terms of 3 service classes described in the column labeled 'Class', and the contracted rates are specified in the column labeled 'Negotiated Rate'. The behavior of the network should be to always promote traffic from a lower class to fill in the lack of traffic in an upper class as specified in the column labeled 'Network Behavior'. For example, at a given time, when the customer's traffic rate is, as shown in the column labeled 'Customer Traffic Rate', $600 \mathrm{kBps}$ for the Gold traffic, 3.8 Mbps for the Silver traffic and 8 MBps for the Bronze, the network should upgrade as much Silver traffic as possible to fill the Gold Negotiated Rate. In this example, it should upgrade 1.4 (2-0.6) MBps of the Silver traffic to Gold, which leaves 2.4 (3.8-1.4) MBps in the Silver and opens up a gap in the Silver Negotiated Rate. The network should then upgrade as much Bronze traffic to fill the 
Negotiated Silver i.e. $600 \mathrm{kBps}(3-2.4)$. Notice that the SLA does not specify what happens to the extra $6.4(8-1-0.6)$ MBps of Bronze traffic that the customer is sending (the provider may choose to forward or drop depending on resource availability).

Table 4 3-tier SLA with automatic upgrades

\begin{tabular}{|l|l|l|l|}
\hline Customer Traffic Rate & Network Behavior & Negotiated Rate & Class \\
\hline $600 \mathrm{kBps}$ & $600 \mathrm{kBps}$ & $2 \mathrm{MBps}$ & Gold (EF) \\
\cline { 2 - 3 } & $1.4 \mathrm{MBps}$ from Silver & & \\
\hline \multirow{3}{*}{$3.8 \mathrm{MBps}$} & $2.4 \mathrm{MBps}$ from Silver & $3 \mathrm{MBps}$ & Silver (AFxy) \\
\cline { 2 - 3 } & $600 \mathrm{kBps}$ from Bron. & & \\
\hline $8 \mathrm{MBps}$ & $1 \mathrm{MBps}$ & $1 \mathrm{MBps}$ & Bronze (BE) \\
\hline
\end{tabular}

Variations on this SLA could include:

- Promote from all lower classes to the upper classes when there is a gap in the upper classes. For example, if there is not enough Silver traffic to fill in the gap in the Gold traffic, promote from the Bronze to Gold.

- Demote the excess Gold traffic to Silver when there is a gap in the Silver traffic instead of dropping it. Or demote the excess from all upper classes when there is a gap in the lower classes.

- Generalization into $\mathrm{n}$ classes instead of 3 classes.

- Promotion/Demotion of any class $m$ into any other class $n$ where class $n$ is a better/lower QoS class then $\mathrm{m}$.

The idea, simply stated, is to always consume the per-class reserved rates instead of dropping the per-class excess even though some rate is available in a different class.

In the next section, we describe what kind of QoS controls can provide such types of SLAs. 


\subsection{Provisioning and QoS controls}

As shown in [69], there exists at least one set of QoS objects (meters, markers, policers, schedulers, etc.) that can provide the SLA shown in Figure 2. We briefly restate the QoS controls that can provide the 3-tier SLA class upgrades (for a more detailed explanation, refer to [69]).

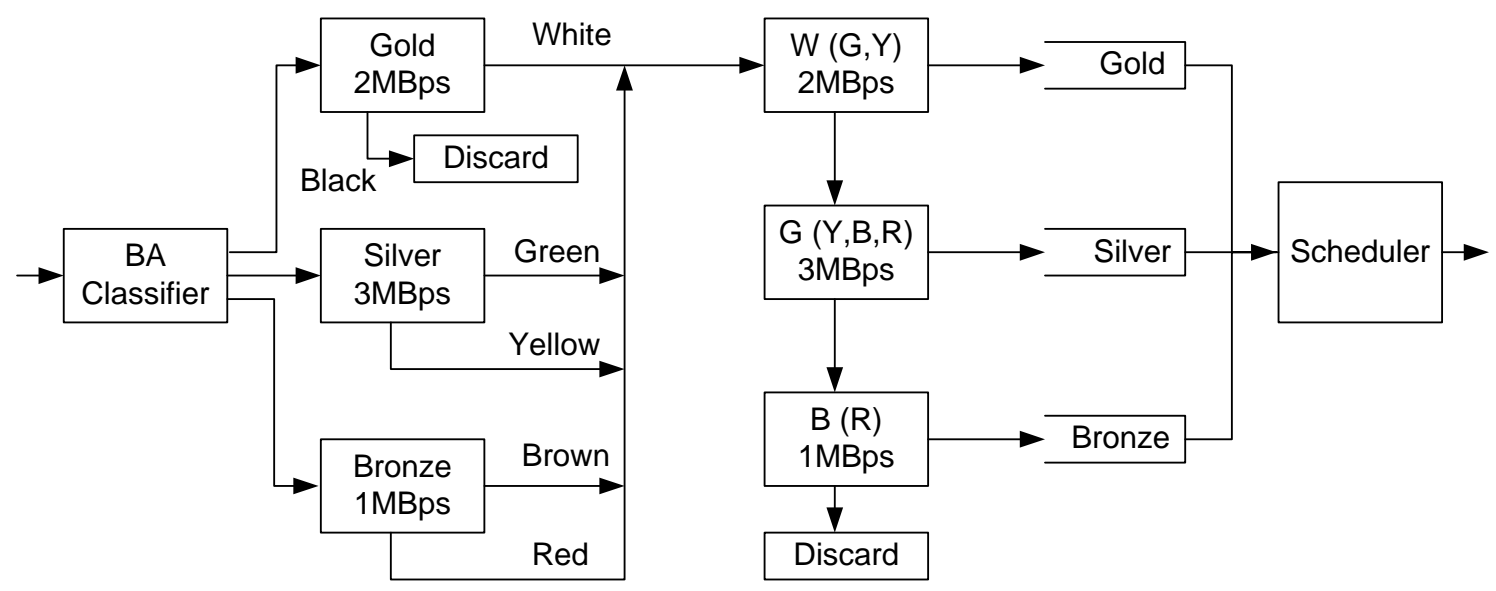

Figure 2 QoS controls for 3-tier SLA with class upgrades

The BA classifier represents a Behavior Aggregate classifier which classifies packets based on the TOS or DSCP field in the packet, and redirects traffic to one of 3 meters labeled “Gold 2MBps", "Silver 3MBps" and "Bronze 1MBps” which meter the customers' Gold, Silver and Bronze traffic to what was negotiated (as specified in Table 4 in this case), respectively. These meters work as follows:

- Gold meter colors conformant (conformant to some certain leaky bucket parameters for example) Gold traffic White, and non-conformant Gold traffic Black (in this case, the excess Gold or Black traffic is dropped).

- Silver meter colors conformant Silver traffic Green, and non-conformant Silver traffic Yellow. 
- Bronze meter colors conformant Bronze meter Brown, and non-conformant Bronze traffic Red.

The output of the first stage of meters is re-inserted into a second level of color-aware policers. This second stage of policers labeled "W(G,Y) 2MBps", "G(Y,B,R) 3MBps", and “B(R) 1 MBps" work as follows:

- $\quad$ The "W(G,Y) 2MBps" policer, counts White packets without policing them (i.e. forwards White packets unconditionally to the Gold queue) and allows Green or Yellow packets into the Gold queue based on both available rate (based on bytes inserted into queue within a period T) and the Gold Queue Size. If there is some Gold bandwidth available, Green (originally Silver) packets are inserted into the Gold queue based on a threshold set for a certain size of the Gold queue. If the queue size is above a certain Green threshold, the Green packet is forwarded to the " $G(Y, B, R)$ 3MBps" Policer otherwise, the Green packet is promoted to Gold and inserted into the Gold queue. Yellow packets observe the same behavior except that the Policer checks a Yellow threshold (where Yellow threshold $>$ Green threshold) to remark and insert a Yellow packet into the Gold queue.

- The "G(Y,B,R) 3MBps" Policer works in a similar manner to the "W(G,Y) 2MBps" Policer in which the Green packets are forwarded unconditionally to the Silver queue and Yellow, Brown, and Red packets are forwarded based on available bandwidth and Yellow, Brown and Red queue thresholds, respectively (where Red threshold> Brown threshold $>$ Yellow threshold).

- $\quad$ The "B(R) 1 MBps" Policer exerts the same behavior with Brown as transparent color and Red as the conditioned color. 
Finally, a guaranteed rate scheduler (for example, WRR or WFQ) guarantees the rate out from the queues as specified in the SLA: 2 MBps for Gold, 3MBps for Silver and 1MBps for Bronze in this case.

Note that the above controls are needed only at the edge of the network where the customer is connected. For more details about the analysis of the components refer to [69].

Figure 3 displays the findings in [69] of the effect of varying the customer's Gold or White traffic on the other classes. The figure shows how much (in Bps) of the Green, Yellow, Brown and Red packets got forwarded as the White traffic varies from 0 to 2.4MBps. As seen in the figure, only the Yellow and Red rates got affected, as they represent packets that would have been promoted if there was bandwidth available in the upper class.

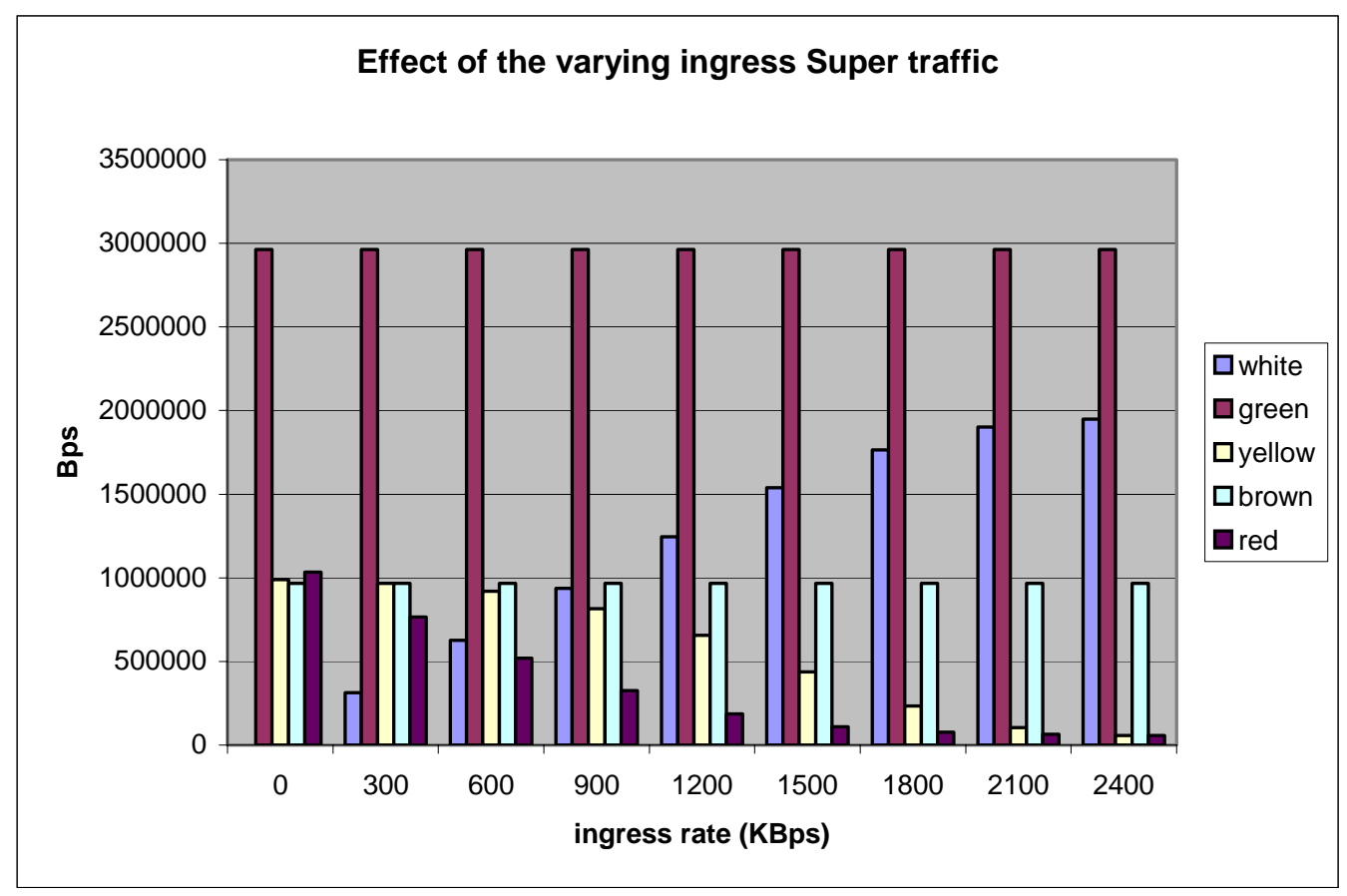

Figure 3 Effect of the Gold traffic 
Figure 4 (also from [69]) shows the effect of the Gold traffic on how the Green, Yellow, Brown and Red exited the node. As the ingress Gold rate is increased, the less of the Green and Yellow traffics got promoted (labeled Super G and Super Y in the figure versus the non-promoted Green and Yellow labeled Silver G and Silver Y) and the less the Yellow traffic got to be forwarded. Also, as the Green and Yellow traffics got less promoted (creating a smaller gap in the Silver reserved bandwidth), the Brown and Red got less promoted as well (shown as the Silver B and Silver R in the figure).

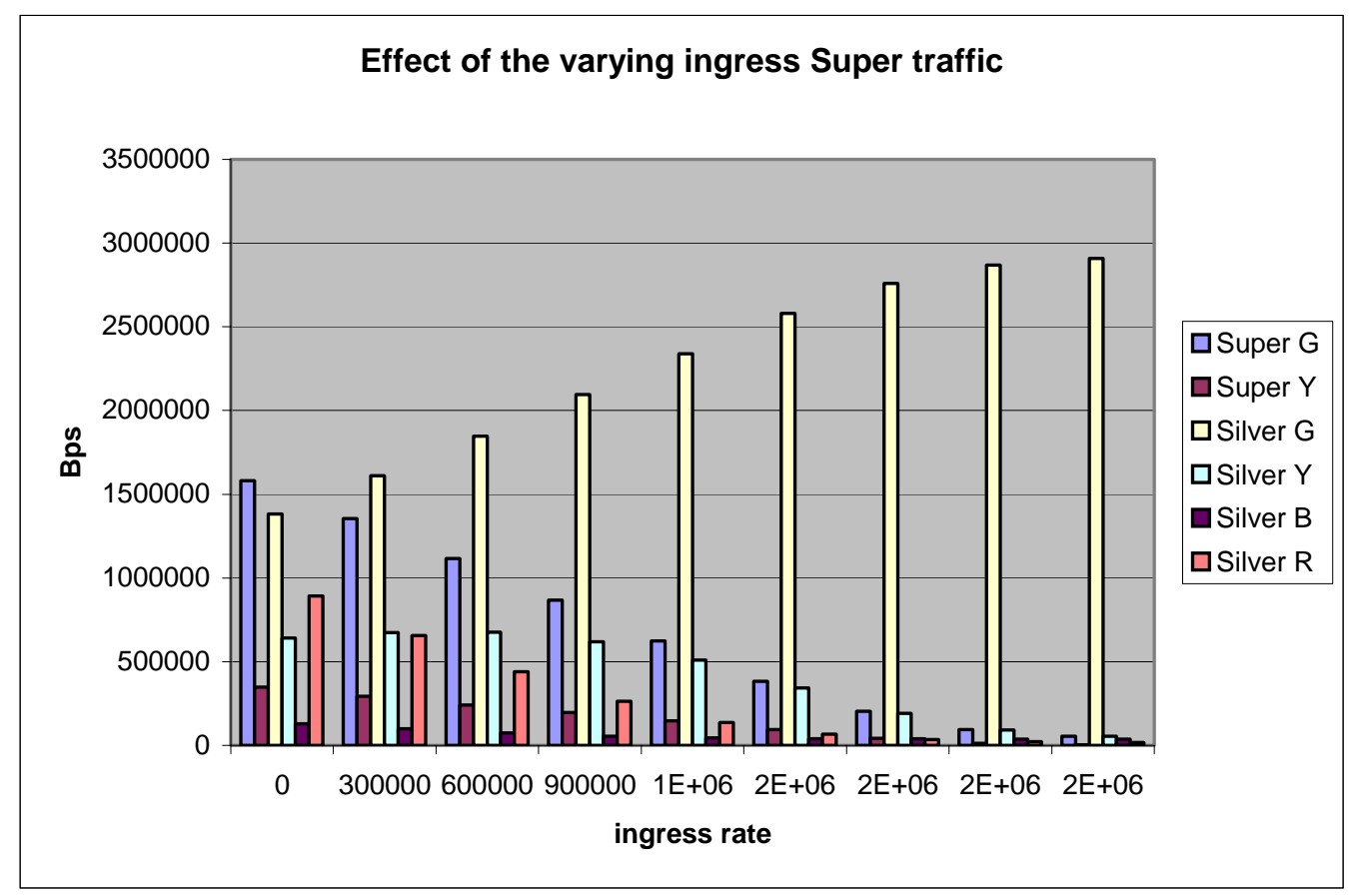

Figure 4 Effect of the Gold rate on other classes

\subsection{Reordering caveat}

As mentioned in [69], the above mentioned QoS controls may reorder packets of the same original Silver or Bronze classes (since this SLA does not involve Gold remarking, 
Gold traffic can not be reordered due to QoS treatment -assuming no reordering due to routing). For example, for two Silver packets inserted at the edge node, depending on the availability of the Gold bandwidth, the second Silver packet may get remarked or upgraded into Gold. Since the second packet will observe Gold treatment until it reaches the end node (receiver or destination), the second packet may arrive at the destination before the first one.

Packet reordering is strongly discouraged mainly due to performance impact of some existing transport protocols [70]. For example, TCP's fast retransmit optimization assumes that packet reordering is sufficiently rare and any reordering event spanning more than a few packets implies a loss. Since TCP also assumes that losses are primarily caused by buffer overflows, these reordering events can be misinterpreted as congestion signals and cause the sender to dramatically reduce its throughput $[73,74]$.

It was shown that large scale reordering can lead to self-reinforcing poor performance from TCP. In fact, forward path reordering (versus reverse path reordering or acknowledgments reordering) causes unnecessary retransmissions, makes it difficult to grow TCP's congestion window, causes packet losses to be obscured, causes the round-trip estimator to poorly estimate its round-trip time, and reduces the efficiency of the receiving TCP. Reverse path reordering causes loss of self-clocking leading to highly bursty transmission patterns: if acks are reordered there may be only one or two acks that acknowledge new data, even if tens or hundreds of segments have been sent. This results in the congestion window growing too slowly (TCP congestion window doubles for every received ack that acknowledges previously unacknowledged data) [79]. [73, 81] propose alternatives to dynamically make the TCP fast retransmission algorithm more tolerant to reordering, in order to avoid the performance problems caused by it. For wireless networks, 
where throughput can be a bottleneck, packet reordering can seriously impact the performance of the network.

Similarly, interactive streaming media protocols, such as Voice-Over-IP, assume that sequencing errors are sufficiently rare that media playout buffers are only necessary to absorb packet jitter [75].

Some protocols incorporate compression on non-idempotent state transitions that can produce inconsistent results in the presence of sequencing errors [76,77].

[78] provides some measurement techniques that can reliably estimate one-way endto-end reordering rates to and from arbitrary TCP-based servers.

The high complexity and high cost associated with handling per flow reordering in some network technologies [71,72] led to recommending non-reordering per hop behaviors (i.e. not to reorder packets within a flow as much as possible). 


\subsection{Solving Reordering Due to QoS Class Remarking}

As mentioned in the previous section, the reordering problem exists not only in the 3tier SLA with automatic class upgrades but also in all SLAs that upgrade or downgrade packets from one service queue to another. Note that this phenomenon does not occur in some SLAs where packets are remarked to other service classes (versus queues) where packets are queued in the same original service class queue (example of such services are the DiffServ AFx classes, where packets are remarked from and to the same AFx class: e.g., AFx1 to AFx2).

As shown in Figure 5, a source communicates with a destination via multiple routers/nodes via some network. Each router is assumed to include a queuing system, consisting of some implementation of logical or physical queues and a scheduler, which stores and implements a queuing method. Legacy queuing methods do not take care of the reordering problem and basically work as follows.

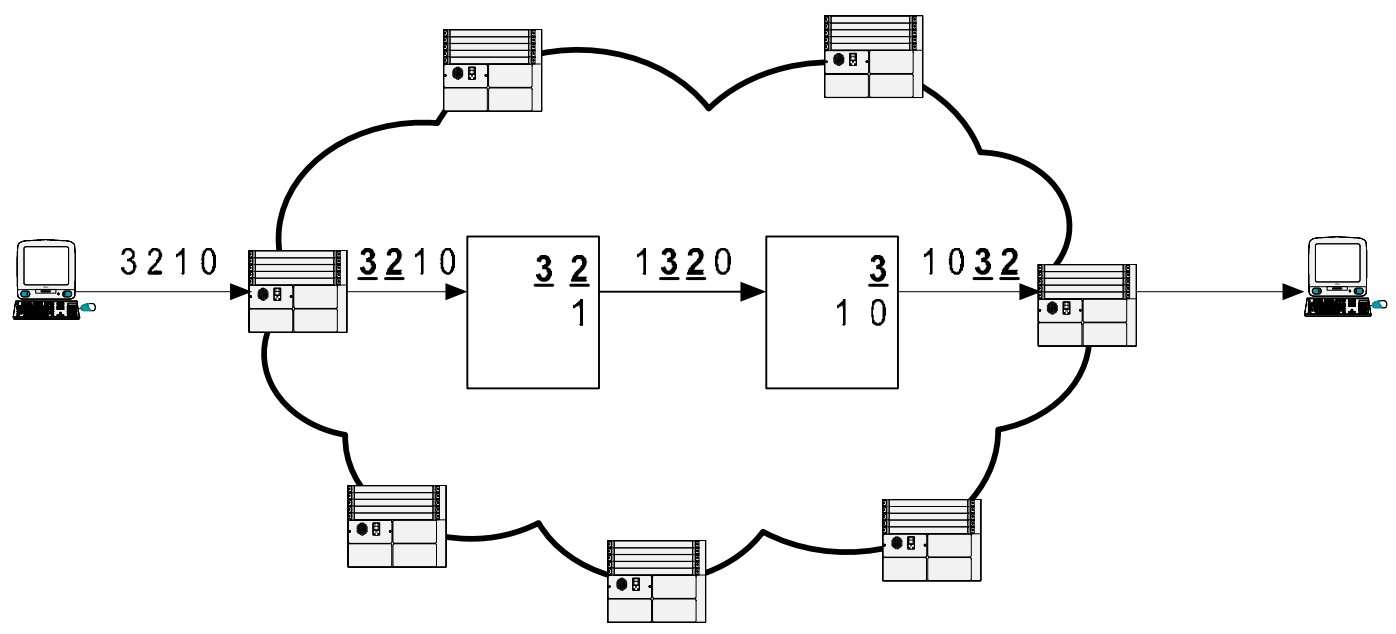

Figure 5 A reordering Network 
Assume that the edge router remarks some packets within a certain flow (the numbers in the figure represents packets' relative positioning to previously forwarded packets that do not intervene in the presentation of this example), in this case packets 2 and 3 are upgraded (shown in a bold and underlined font after being upgraded in the figure). Depending on the scheduling scheme and the state of the various service queues in downstream nodes, packets 2 and 3 (since they are upgraded to a better service class) can arrive at the destination before packets 0 and 1. For example, one scenario is that the first downstream node could schedule packets 2 and 3 before packet 1 since they are of higher service class, and the next downstream node could schedule packets 2 and 3 before packet 0 .

In order to maintain the ordering criteria within a flow, nodes could decide to maintain a per-flow state that keeps track of packet sequence numbers and queue out of order packets until they are back in order. This solution is not desirable, since it would not scale when the number of flows becomes large; it would require keeping state information for every flow $[71,72]$.

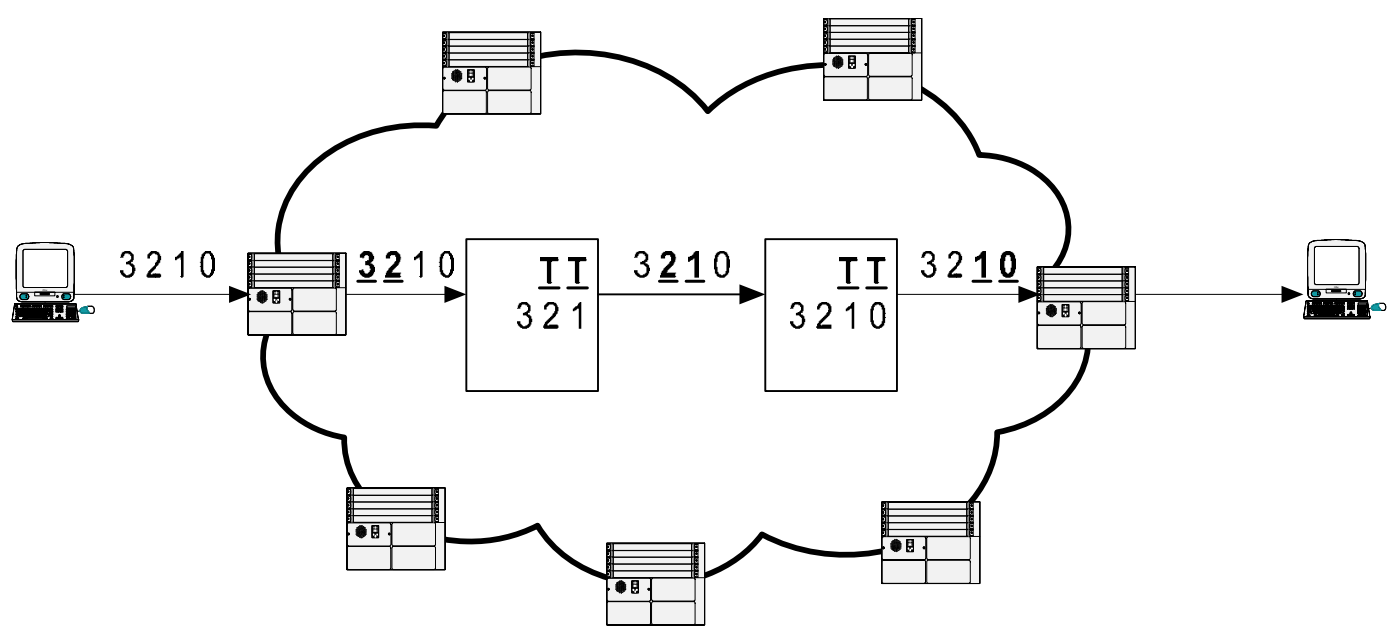

Figure 6 A non-reordering Network 
The proposed solution is to force each node to maintain packet ordering as follows. Use a special indicator in each packets' header (e.g. one of the DSCP bits) to tell a downstream node that a packet has been upgraded to a higher class (from CLASS-2 to CLASS-1 for example). Once the downstream node detects a packet with the special indicator set, it would queue the upgraded packet in its original class of service (CLASS-2), reset the special indicator, and add a token in the higher class (CLASS-1) to represent the upgraded packet. Once the scheduler decides that the token packet needs to be forwarded, it would forward instead the head-of-line packet from the lower class (CLASS-2) and set the special indicator in that packet. The idea is similar to the 400 meters relay, where each runner runs 100 meters and relays a baton to the next runner.

Figure 6 depicts an example of the proposed solution which contrasts the previous example. As shown in the figure, when packets 2 and 3 (after being upgraded at the edge router) are received in the first downstream node, they are placed back in their original class of service queue, and tokens represent their arrivals as upgraded packets in the higher service class. If packet 0 was forwarded before it has the chance to be upgraded, as shown in the figure, the next packet in the lower class i.e. packet 1 will be upgraded with the special indicator set (thus consuming a token). Assuming no reordering in the line between two nodes, packet 0 will arrive first (since it was transmitted first) to the downstream node. The figure also shows how the second downstream node upgrades the head-of-line packets from the lower class (in this case packets 0 and 1 ) upon scheduling received tokens (from packets 1 and 2 in this example).

The downstream nodes fake the presence of a remarked packet in the higher QoS class by allocating for example a proxy packet or maintaining a counter of packets/bytes 
received (implementation dependent) in the higher QoS class (by maintaining a byte count, the scheduler can preserve the quality of service observed for the flow). This is done so that the scheduler can service a lower class packet when it is time to service the proxy packet (or token).

The special indicator described above can be implemented through a packet header field value or a bit. For example, in Diffserv it could be a 'special' DSCP (Differentiated Services Code Point) value that indicates for instance that this packet was AF2: Assured Forwarding 2 (A Diffserv Quality of Service Class) and is promoted to AF1: Assured Forwarding 1 (Another Diffserv Quality of Service Class). And, the creation of the proxy packet can depend on the implementation of the queuing and scheduling system in hardware. In one instance, it can be a pointer to the head-of-line packet of the second class; when the scheduler decides to schedule the proxy packet, the head-of-line packet of the second class is remarked and transmitted.

Note that the above solution to the reordering problem was filed as a patent application to the US patent office in September 2004.

\subsection{Reordering Solution Proof}

In order to provide a formal proof we make the following assumptions:

1. Reordering can happen only in a forwarding node; no reordering can happen on the wire or line.

2. Reordering can happen only due to queuing/scheduling; no reordering can happen in the classification logic which decides in which queue each packet is to be inserted.

3. No reordering can happen within a service queue; we assume FIFO queues. 
4. Within a node, packets of the same service class traverse the same path from Input to Output (e.g. across the switch fabric). Also, the solution is applied at the earliest point possible in the node where reordering can happen due to service class queuing (e.g. packets are remarked to the original service class and tokens are inserted in the higher class before they (packets) cross the switch fabric on a different quality of service path).

5. No reordering can happen due to routing; this solution does not solve reordering caused by routing.

6. Packets of the same flow are originally marked with, or upgraded/downgraded to, the same code points (i.e. will be classified into the same service queues).

7. No reordering in customer equipment (reordering can occur only in the network).

8. A node can be modeled as a sequence of 3 stages: ingress processing, switch fabric, and egress processing, as shown in Figure 7. In turn, each stage (ingress, fabric, or egress) can be modeled in terms of a sequence of queuing and scheduling stages (not shown in the figure).

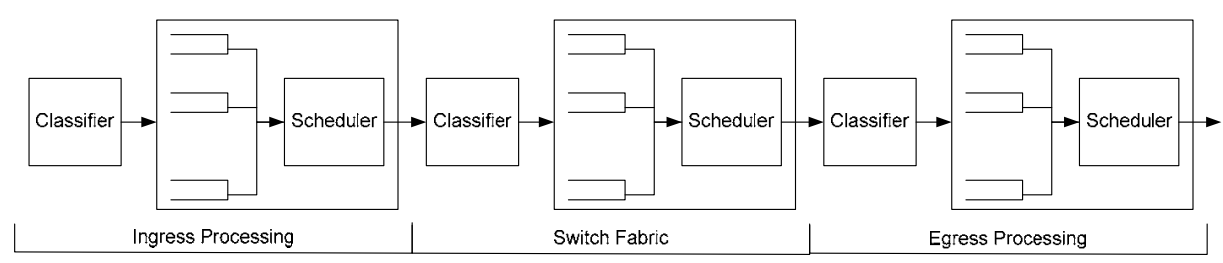

Figure 7 General Node Model 
Given the above assumptions, we can model the path from Figure 6 between the first ingress edge node and the last egress edge node as a sequence of classification (Multi-field or aggregate), and queuing-scheduling stages as shown in Figure 8.
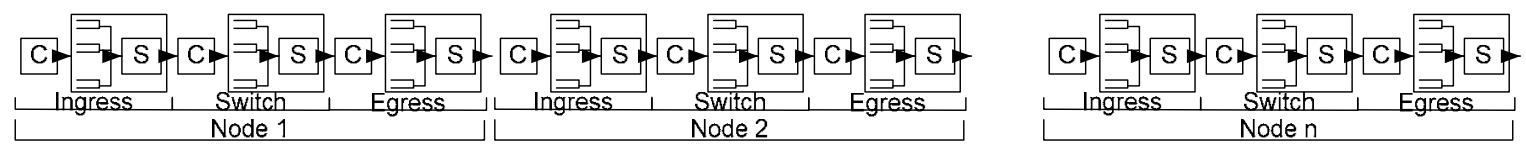

Figure 8 Multi-node model

From assumption 2, reordering can happen only in a queuing-scheduling stage (there are $\mathrm{N}$ in the figure; a sequence of $\mathrm{n}$ nodes, each having $\mathrm{m}$ queuing-scheduling stages). We need to prove that reordering can not happen in the first queuing-scheduling stage, and that given no reordering in stage $\mathrm{i}-1$ there will be no reordering in stage $\mathrm{i}$.

At the first node, the classifier classifies the packet into a verification stage (not shown in the figure) that polices the customer's traffic. Packets can get remarked, metered and policed but not reordered. Two sequential packets of the same original service class will be inserted in the same order of arrival in the same service queue since:

- If none of the packets got upgraded/downgraded, they will be inserted in the same service queue.

- If any of the packets got remarked, a special indicator will be set in the remarked packet and will be inserted in the same service queue as the other (keeping the same order). A token will be added in the higher/lower queue to indicate that a packet was promoted/demoted.

Since we assumed no reordering within the same service queue (assumption 3), both packets will be scheduled to exit the queuing-scheduling stage in their order of arrival. Thus 
in the first queuing-scheduling stage, reordering can not happen (i.e. packets originally in the same service class will be forwarded in the same order of their arrival).

Assuming that there was no reordering of packets within the same service class in the $(\mathrm{i}-1)^{\text {th }}$ queuing-scheduling stage, there will be no reordering in the $\mathrm{i}^{\text {th }}$ stage either since:

- First case: arrival of 2 packets of the same service class with same special indicator (either set or not set for both). In this case, both packets will be classified into the same service class, and thus will be scheduled in order.

- Second case: arrival of 2 packets of the same service class but different special indicator (set for one but not the other).

o If the first packet has the special indicator set, then it will be unset, the packet inserted in the original service class, and a token will be inserted in the upper/lower class. The second packet will be inserted after the first (since it is received after the first) in the same service class.

o If the second packet has the special indicator set, then it will be unset, the packet inserted after the first (since the first was already received) in the same service class and a token will be inserted in the upper/lower class.

Notice that the insertion of a token insures that the promised Quality of Service is maintained, by "handing over" the packet's new Quality of Service level to the head-of-line packet of the same original service level, while maintaining the order of packets. 


\section{Chapter 6}

\section{Experimental Results -}

\section{3-Tier SLA Performance}

We have presented in Chapter 5 a 3-tier SLA with automatic upgrades, and a solution to the reordering problem due to packet promotion. We have provided a proof to the reordering solution, and argued that it allows for certain Service Level Agreements that embed class upgrades or downgrades.

In this chapter, we focus on the network analysis of the 3-tier SLA with automatic upgrades and we further investigate its benefits in terms of throughput, goodput, delay and loss by comparing the simulation results of 3 models: the 3-tier SLA with no upgrades (we will call it DS after the current Diffserv model), the 3-tier SLA with automatic upgrades but without the reordering solution (we will call it SLAR), and finally the proposed 3-tier SLA with automatic upgrades and with the reordering solution (we will call it SLA).

We first describe in section 6.1 the simulation environment and the developed simulator classes and modules, give an overview of the Gold, Silver and Bronze service 
classes' expected behavior, and talk about general simulation information like confidence interval computation. In section 6.2, we present the network topology used in this chapter and explain the configuration of the various network parameters. In section 6.3 we focus on the ingress class upgrades and show that without the reordering solution the upgrades provide not as good of a service. In section 6.4 we compare the behavior of the 3 SLA models (DS, SLAR, SLA) in terms of QoS performances (throughput, goodput, loss and delay), and we also show the behavior under various network load and various packet sizes. In section 6.5 we highlight possible implementation issues on Intel's 28xx Network Processor firmware using Ericsson's ISER Edge Router. Finally in section 6.6, we give an overview of this chapter results, highlighting the benefits of the proposed SLA.

\subsection{Simulation Environment}

One widely used network simulator is NS-2, provided by the University of Berkeley. NS-2 is a discrete event simulator targeted at networking research. It provides substantial support for simulation of TCP, routing, and DiffServ over wired and wireless (local and satellite) networks. The code (open source) is written in $\mathrm{C}++$ and network topologies and simulations are driven by a TCL interface. Various contributors to NS include Nortel, Bell Labs, various universities and individuals.

We will first start by describing the newly developed DiffServ NS-2 module that allows switching between the various SLA models, then we will talk about the setup of the Gold, Silver and Bronze traffic classes and finally we will display some general information concerning the simulation results. 


\subsubsection{New NS-2 DiffServ module}

In order to study the SLA, a new NS-2 DiffServ module, composed of several C++ classes, was developed. The module is object-oriented with well defined interfaces between objects. It allows easier replacement of several of the objects like meters, schedulers, packet buffers, etc. We are hoping to contribute this module back to the NS community.

We will define in the following paragraphs the key components that are crucial for the SLA achievement and the reordering solution.

\subsubsection{Egress Queuing and Scheduling}

The egress queuing at each node is modeled after a DSQueue $\mathrm{C}++$ class. The DSQueue consists of three classes: a classifier, a packet buffer and a scheduler with a well defined interface between them in order to allow interchangeability of these inner classes. The classifier can be a multi-field classifier typically used on ingress nodes, or a Behavior Aggregate classifier typically used in internal nodes. The packet buffer class is composed of objects necessary for building a certain service (e.g. a policer followed by a FIFO queue). For example, one of the defined packet buffer classes contain an array of DSQueues, each having a classifier, a packet buffer and a scheduler (the edge node described below is an example of such a DSQueue). And finally, the scheduler defines an algorithm to determine which of the queues is to be serviced next.

The ingress edge node, as shown in Figure 9 below, is composed of a Multifield classifier, a 'DSPktBufSLA' packet buffer and a Weighted Round Robin (WRR) scheduler. The 'DSPktBufSLA', in turn, is composed of 5 DSQueues (one per client in the first scenario), each composed of a Behavior Aggregate classifier, a 'DSPktBufDSM' packet buffer and a WRR scheduler (as it is observed, the edge node has a 2 hierarchy of 
schedulers). The 'DSPktBufDSM' is composed of 3 sets of Meters, Policers and FIFO queues (one per traffic class) as seen in the figure.

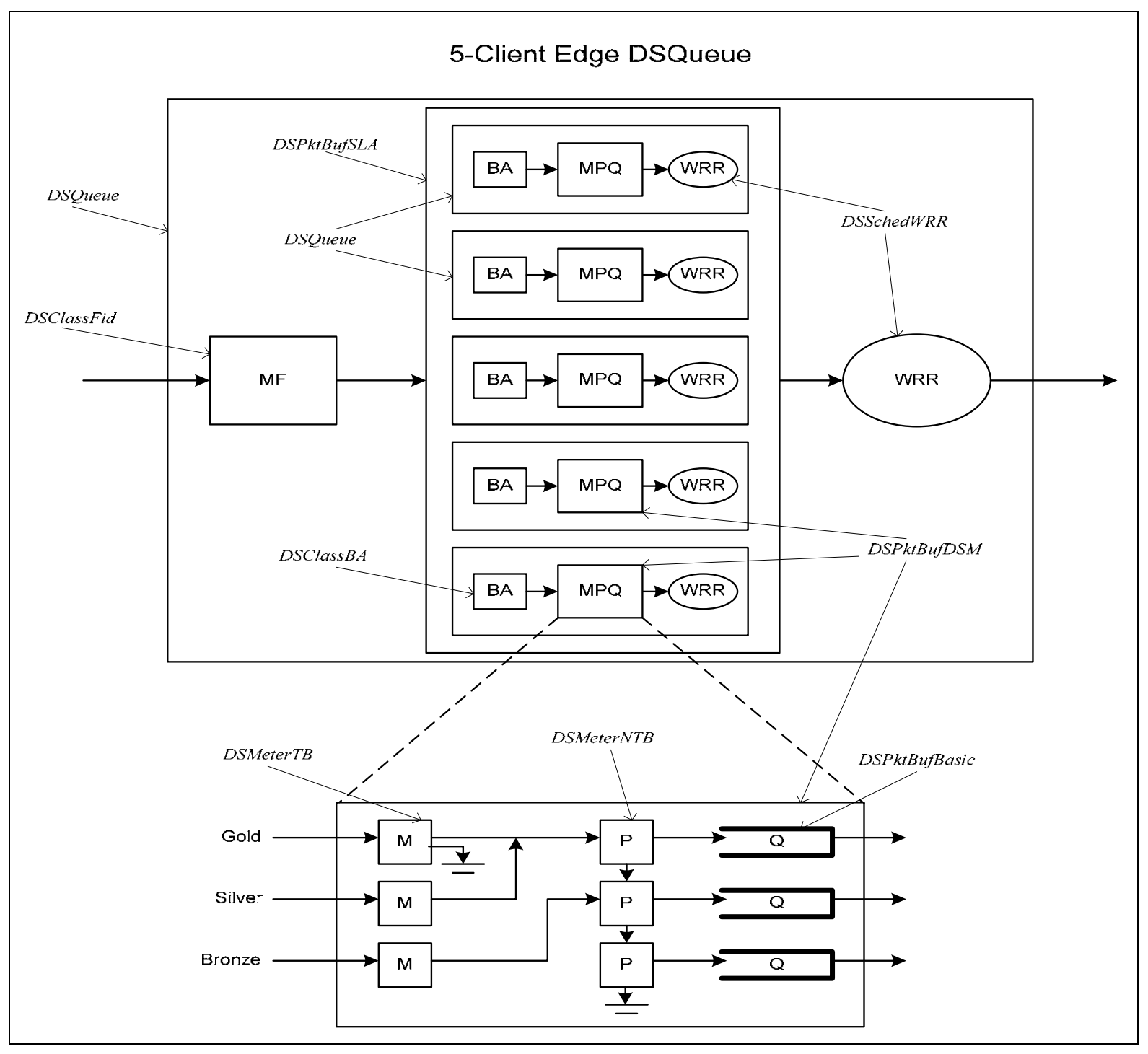

Figure 9 Ingress edge node DSQueue model

At the edge node, ingress packets are presented to the MF classifier, which separates each client's traffic for further inspection. For example, a particular client's traffic will be 
classified into one of the inner DSQueues (the MF classifier will be configured to identify that client and forward its traffic accordingly). The traffic is then BA classified, in the inner DSQueue for traffic class separation, and presented into one of the meters in the DSPktBufDSM, for example the silver traffic will be presented to the second meter which is configured to meter and color the customer's traffic based on conformant and nonconformant traffic (leaky bucket with CBS, CIR parameters). After the metering stage, the traffic is presented to a "policing" stage that is color-aware and could remark/upgrade the traffic. The traffic is then forwarded, if not dropped, into a FIFO queue waiting to be serviced. When the first level WRR decides that a packet from this client is to be serviced, the second level WRR decides which of the customer's service queue is to be serviced. The first level WRR guarantees rate per customer whereas the second WRR guarantees rate per service class. This hierarchy of schedulers is needed to guarantee rate per service class per customer.

The MPQ box (M, P, and Q represents the 3 successive stages of metering, policing, and queuing, respectively) shown in Figure 9 polices a particular customer's service class (Gold, Silver, Bronze) according to a preset rate, and allows lower-class upgrades whenever the upper-class reserved rate is not fully consumed (this is achieved in the DSMeterNTB class described below). Gold traffic enters into the Gold M (excess Gold traffic is dropped here), into the Gold P, and then into the Gold Q. Silver traffic enters into the Silver M, into the Gold P, possibly into the Gold Q (if upgraded) otherwise into the Silver P, into the Silver Q or dropped. And finally, the Bronze traffic enters into the Bronze M, into the Silver P, possibly into the Silver Q (if upgraded) otherwise into the Gold P, into the Gold Q or dropped. Here are the details of the MPQ box: 
- The Gold traffic is first metered by a leaky-bucket (CBS, CIR); Gold traffic conforming to the leaky bucket is forwarded to the next stage $(\mathrm{P})$, whereas nonconforming Gold traffic is dropped. The conforming Gold traffic is then policed in the P stage and forwarded to the Gold Queue. The Gold P stage merely counts Gold traffic in order to decide whether Silver traffic can be upgraded (whenever Silver traffic is forwarded into the Gold P). The P stage is another leaky bucket stage where the number of tokens in the bucket is allowed to go negative (the maximum negative value occurs when a burst of Silver packets consume all tokens followed by a burst of CBS Gold packets - since Gold M allows up to CBS burst, the maximum negative value is -CBS). Gold traffic going into the P stage is not conditioned; it just updates the P stage tokens so that when Silver traffic is conditioned by the same P stage it is either upgraded based on whether there is enough tokens in the P stage bucket or not. So the Gold P stage is actually a special Policer for the Gold and Silver aggregate which always allows Gold but conditions Silver based on token availability. Silver packets inserted in the Gold Q are actually upgraded packets.

- The Silver traffic is first metered into conformant and non-conformant, both of which will be forwarded to the Gold P. If there are enough tokens in the Gold P bucket, the Silver packet (conformant or not in the Silver M stage) will be upgraded. However, conformant Silver packets that are upgraded are counted so that whenever a nonconformant Silver packet passes the Gold P down into the Silver P, it gets re-colored as conformant (otherwise, the non-conformant Silver packets will be replaced by upgraded Bronze packets at the Silver P stage whenever a conformant Silver packet is upgraded to Gold). A simple example is a sequence of 1 conformant Silver packet, 1 
Bronze packet, and 1 non-conformant Silver packet. Assume the conformant Silver packet gets upgraded to Gold, the Bronze packet got upgrade to Silver, then the nonconformant Silver packet may be dropped at the Silver P in case the Bronze packet empties the tokens in the Silver P. This behavior is fixed if the non-conformant Silver packet is re-colored as conformant and the Silver P never conditions the conformant Silver packets (same behavior as the conformant Gold with the Gold P). Again, in this case, the Silver $\mathrm{P}$ is allowed to go negative in tokens (up to -CBS).

- And finally, the Bronze P drops the excess Bronze packets.

The P stages can be configured to upgrade or to not upgrade (no upgrade falls back into the DiffServ model). They can also be configured to generate tokens (packet tokens required in the reordering solution); upgrade without packet tokens implies network reordering of packets. The proper configuration of this class implies the Diffserv model, the traffic class upgrade SLA with reordering, or the SLA without reordering.

\subsubsection{Negative Leacky Bucket}

The DSMeterNTB class is based on a leaky bucket algorithm, with CBS and CIR parameters, which allows the bucket tokens to go negative as much as -CBS. It differentiates between 4 different colors (of traffic): invisible, non-conformant, visible, and critical. It relies on the previous stage of Meters to color 2 of the traffic classes (e.g. for the Silver DSMeterNTB, Silver and Bronze traffic will be observed) into one of those categories based on the initial service class and whether that class was conformant or not in the first stage. The invisible and non-conformant colors apply to the same traffic class that this DSMeterNTB is assigned, and the visible and critical colors apply to the lower class that this DSMeterNTB need to assess for upgrade. For example, for the Silver DSMeterNTB, the first stage 
conformant Silver traffic will be invisible, non-conformant Silver traffic will be nonconformant, conformant Bronze will be visible and non-conformant Bronze will be critical. This class is best described by a snippet of the code as shown in Table 5 .

Table 5 DSMeterNTB code snippet

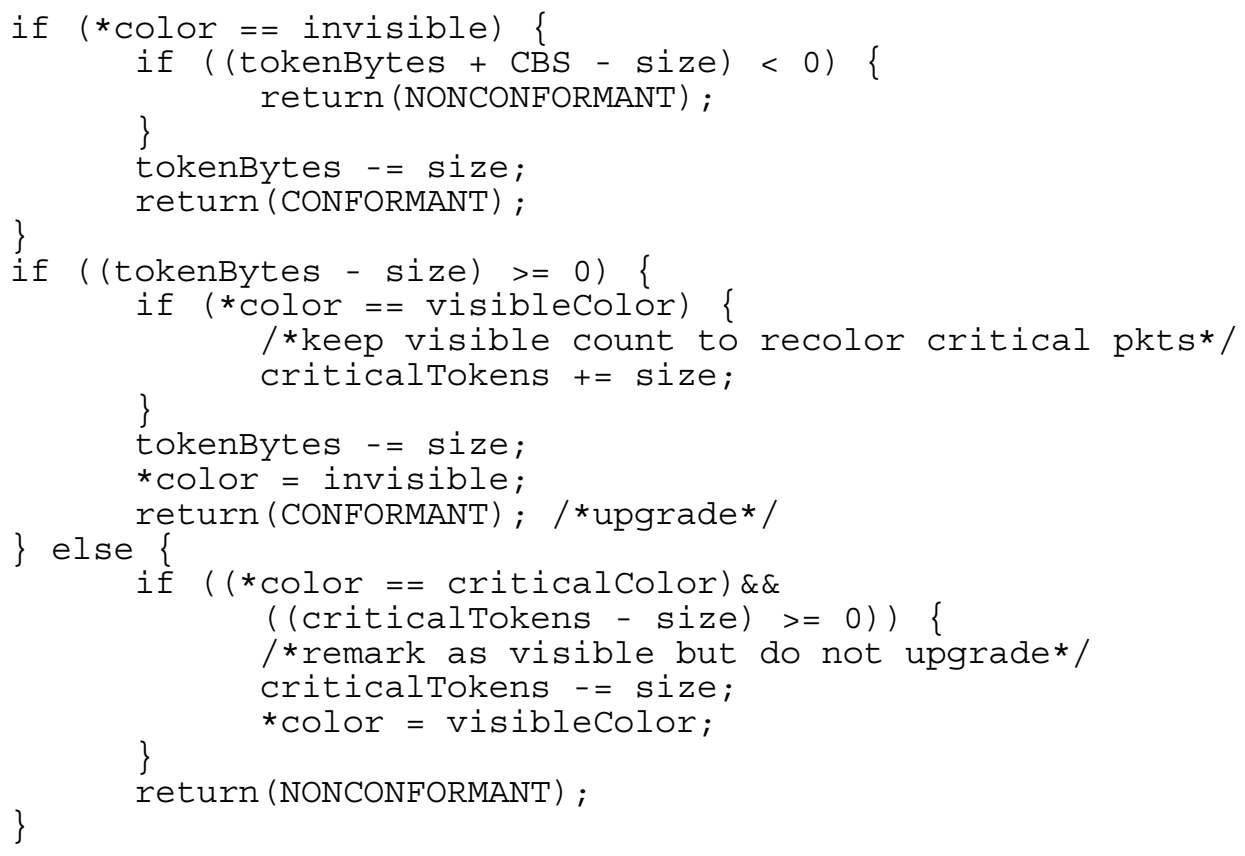

The code shows that if the color is invisible and there are enough bucket tokens (up to -CBS), the packet is inserted in the queue (packet deemed CONFORMANT, and is not dropped). Therefore, only invisible color packets can drive the bucket tokens to go negative.

For the 3 remaining colors, if there is enough tokens (tokenBytes-size $>=0$ ), the packet is inserted in the queue and thus automatically upgraded in case the color is visible or critical. If there aren't any bucket-tokens remaining, the packet is either dropped in case it is a nonconformant packet or could not be upgraded if it was a lower class packet (the caller of this function acts accordingly based on the NONCONFORMANT return code). 
This class keeps track of the number (in bytes) of visible packets upgraded so that it re-colors critical packets as visible in their place whenever a critical packet is nonconformant to the second stage. This is done so that when the lower class traffic is forwarded down to the next DSMeterNTB, it doesn't get dropped due to competing $2^{\text {nd }}$ lower class traffic that is trying to get upgraded. For example, if the Gold DSMeterNTB does not recolor non-conformant (to the first stage) Silver traffic as conformant (in case previously some conformant Silver traffic was upgraded to Gold), when that Silver traffic is forwarded to the Silver DSMeterNTB for assessment it will compete with the Bronze traffic which could force it to be dropped (since it is still non-conformant Silver traffic) depending on the Bronze rate (Bronze stealing bandwidth from the upper Silver class).

For the DiffServ case, the algorithm described above is omitted, i.e. if the first stage labels the traffic as non-conformant it will be dropped (second stage is omitted).

\subsubsection{Packet Buffer}

The DSPktBufBasic class enqueues and dequeues packets from or into one of 3 FIFO queues (for the 3 traffic classes), and implements the RED algorithm for buffer management (each queue is configured separately). It queues packets passed from the second stage DSMeterNTBs, and dequeues packets whenever the scheduling algorithm requests so (refer to Figure 9).

In case the class is configured to be token-aware (this scheme was described in section 5.4), it checks whether a bit in the packet header is set to indicate that this packet was upgraded. If the packet was upgraded as determined by the DSMeterNTBs, it then creates a token packet (a placeholder in the queue) and inserts it in the upgrade class but inserts the physical packet in its original class (to preserve ordering). When the scheduler requests to 
dequeue a certain queue and a token packet is found, the lower class is dequeued instead, and the dequeued packet is upgraded (the indication bit is set in that packet). This implementation keeps the scheduler unaware of the token implementation (easier to change the scheduler).

Note that the token awareness is turned off for the reordering SLA and the DiffServ models.

\subsubsection{Service classes}

In the simulations that follow, we assume 3 service classes set up to provide Gold, Silver and Bronze services. Gold Service is targeted for real time applications like voice which assume little queuing delay. It is constructed based on limiting the amount of Gold traffic accepted into the network so that the sum of all Gold flows rates (assuming CBR flows) is less than the guaranteed service rate for the Gold aggregate. In DiffServ for example, the Gold service would be EF which is usually setup to emulate circuit switching. Figure 10 shows a typical gold queue size along with the calculated RED average queue size versus time. 


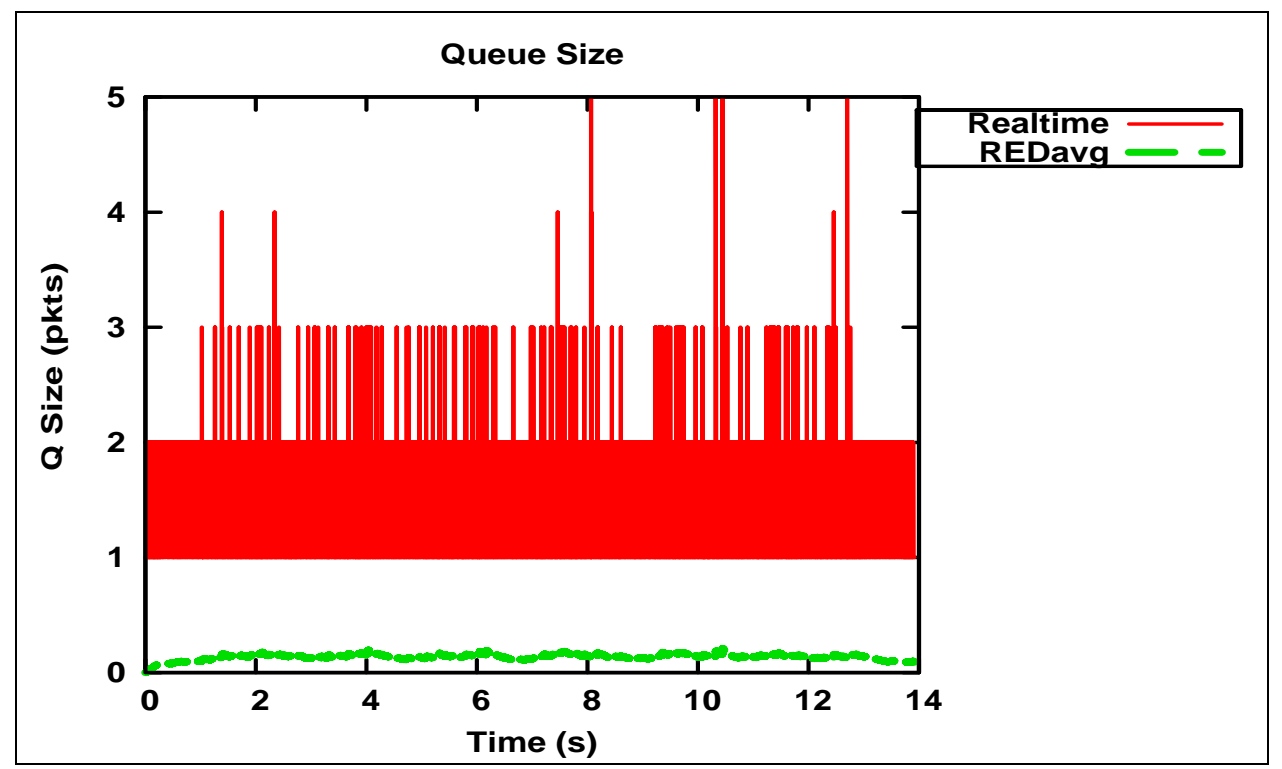

Figure 10 Typical Gold Queue Size

Silver service is targeted for non real time applications that are tolerant to loss but require better than best effort service (e.g. video). It is constructed based on an assured service rate for the Silver aggregate and a buffer management algorithm, RED, which is set up to drop packets whenever the average queue size exceeds a pre-configured threshold. Silver traffic assumes some queuing but smaller queuing than the bronze traffic (better than best effort). Figure 11 shows a typical Silver queue size along with the calculated RED average queue size (assumes a RED max threshold at 20 packets) versus time when all Silver flows are using the maximum allowed rates (worst case condition). Typically the Silver traffic is over-booked (Silver service could be setup so that the sum of all silver flows exceeds $100 \%$ of the reserved link capacity ratio). New clients are allowed into the network as long as the aggregate service provided is still within the advertised QoS of that class. Typically, providers use a CAC algorithm in order to maximize the benefit (i.e. dollars) without affecting the "promised" service for the aggregate i.e. as long as the new incoming 
flow does not affect the "promised" service offered by the Silver class, the flow is accepted even if the sum of the rates of all flows exceed the reserved rate for the aggregate (it builds on the dynamic behavior of the flows and some feedback from the network on whether the new flow would badly affect the Silver Service if accepted).

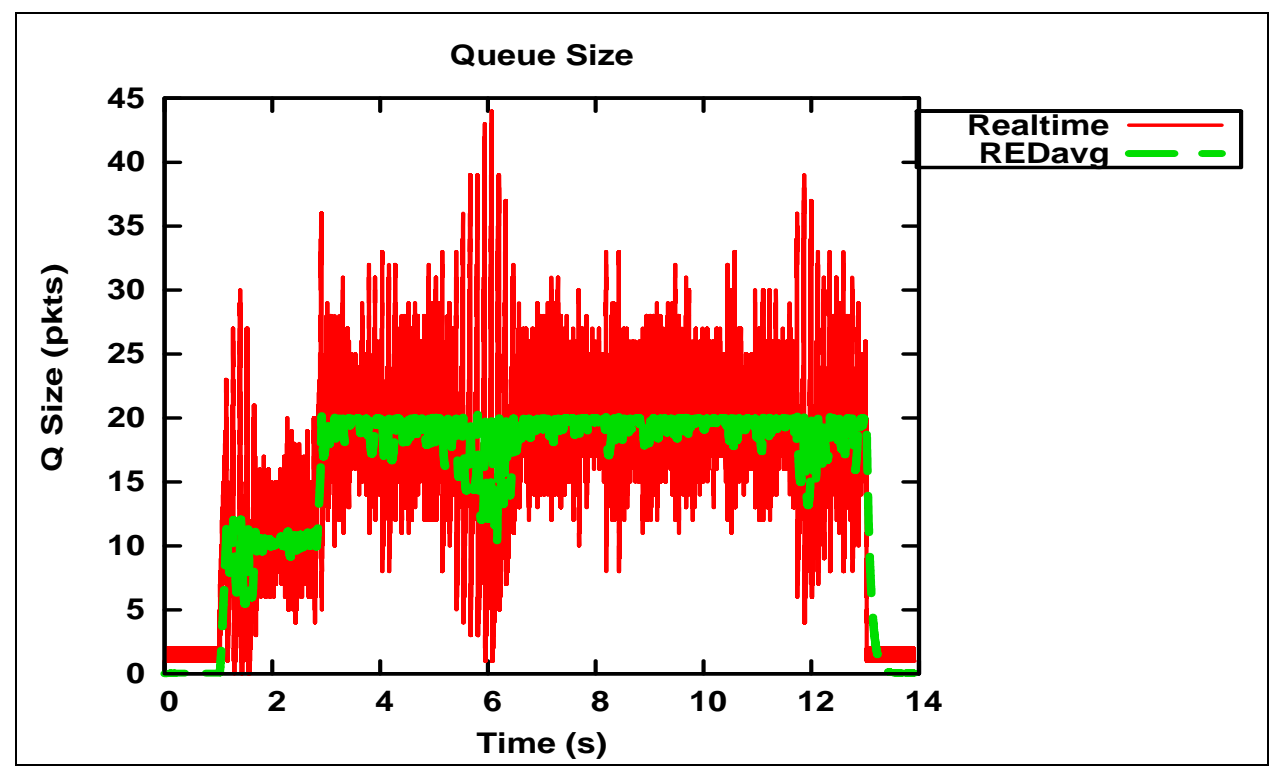

Figure 11 Typical Silver Queue Size

As for the Bronze service class, being a best effort service, it is usually overbooked with higher over-booking percentage than the Silver service (since there are no service guarantees associated with the best effort service class). A buffer management algorithm (e.g. RED) keeps the Bronze queues sizes from exceeding a pre-configured threshold. Figure 12 shows a typical Bronze queue size and the calculated RED average queue size (assumes a RED max threshold at 30 packets) versus time in the worst case traffic scenario. 


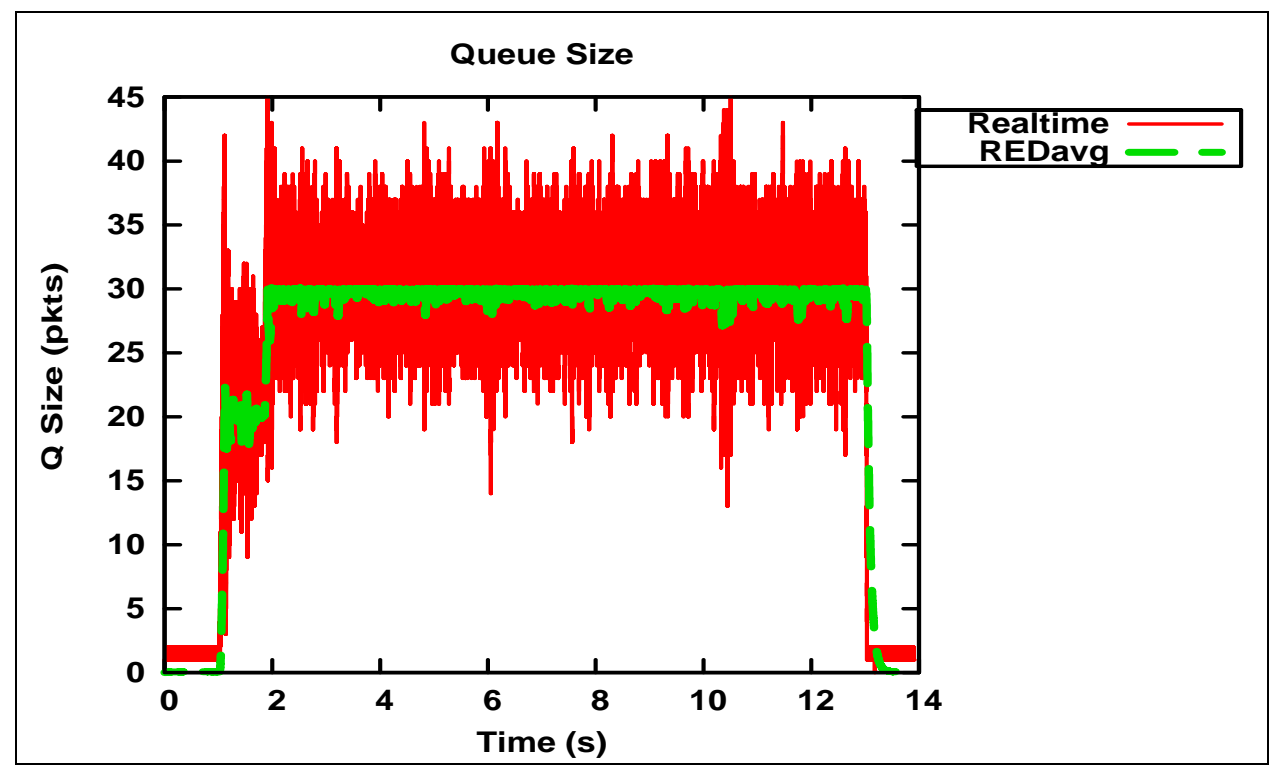

Figure 12 Typical Bronze Queue Size

Figure 13 shows the Cumulative Distribution Functions (CDFs) of the typical worst case End-to-End delay of each of the services (in the figure $50 \mathrm{~ms}$ is consumed by propagation delay over 5 links). Figure 14 indicates that in the worst case condition (network is fully loaded) loss is expected at each node along the end-to-end path for the Silver and Bronze services assuming Silver and Bronze traffic merging at each hop (background traffic). Note that the $y$-axis rate values are provided as an example. The figure shows that Bronze traffic will be more heavily affected than the Silver traffic, whereas the Gold traffic will not be affected (refer to the Gold service setup above). Note that the loss rate is controlled by the RED setup and the amount of Silver and Bronze traffic allowed in the network. 


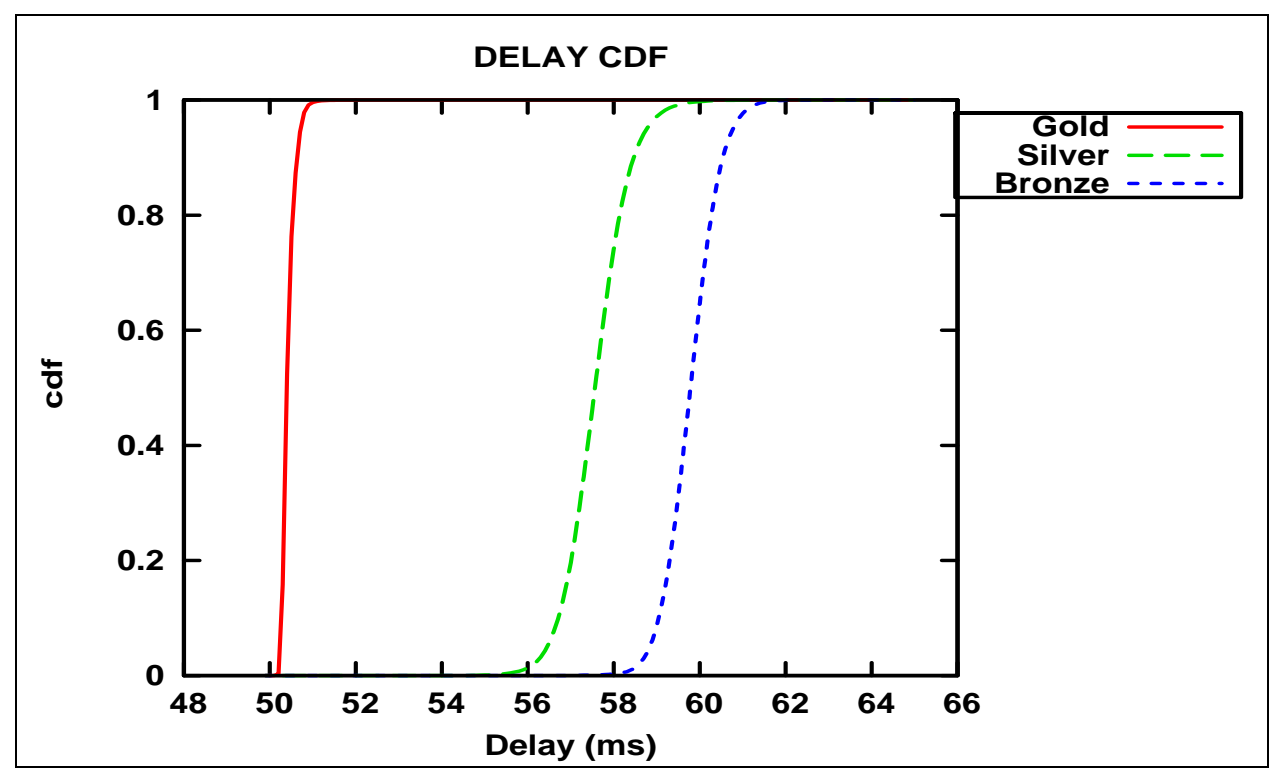

Figure 13 Typical worst case End-to-End delay CDF

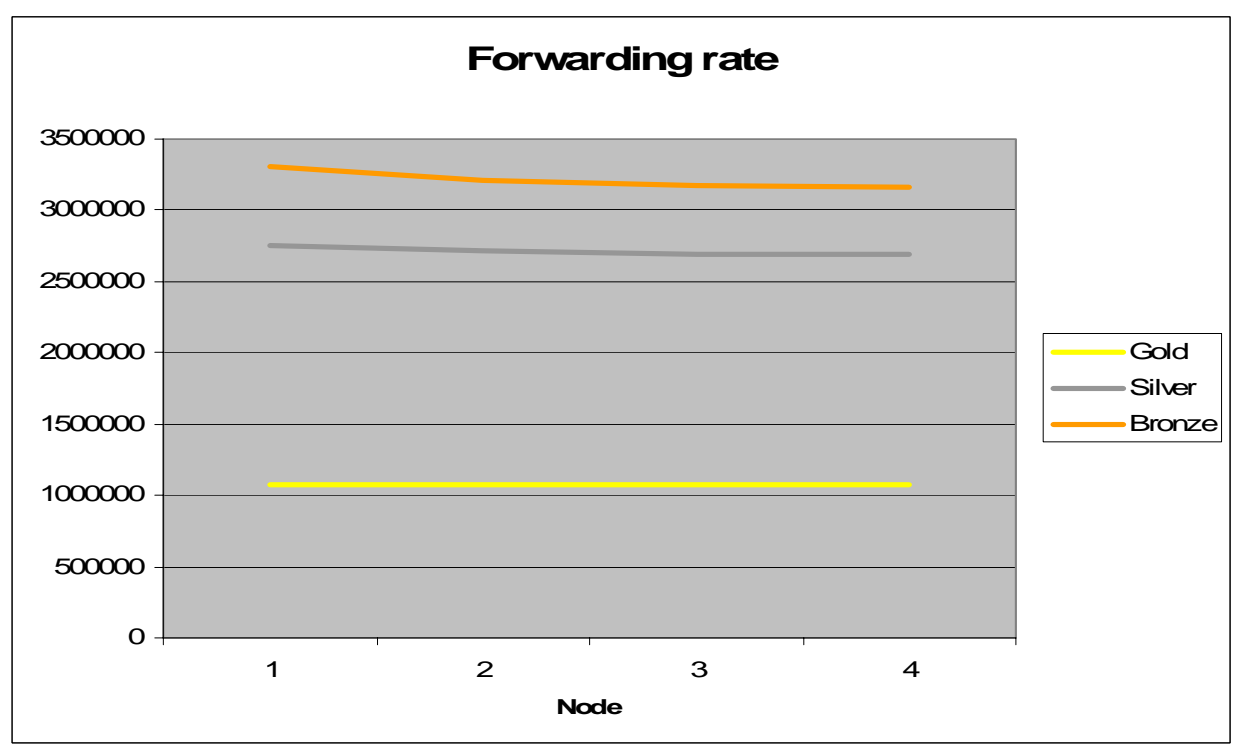

Figure 14 Typical worst case per hop forwarding rate 


\subsubsection{General Information on Results}

\subsubsection{Confidence Intervals}

In the following sections, we use 30 independent observations computed over a steady-state period T, after eliminating transient periods (by letting the model warm up for about $6 \%$ of the sampled time interval) in order to compute $95 \%$ confidence intervals estimated by the method of the batch means [85].

We will show Confidence Intervals in graphs as a vertical bar displayed on the data point collected. Notice that in some graphs confidence intervals are very narrow and as a result are barely visible in the figures.

\subsubsection{Graph Label Notation}

In the following graphs, we will use the generic labeling " $\mathrm{x} / \mathrm{y} / \mathrm{z}$ " to define a certain curve or set of data as shown in Figure 16 in the upper right hand corner of the figure. The first word of the label ( $\mathrm{x}$ in this case) defines the simulation case, for example 'DS' for DiffServ or 'SLAR' for the 3-tier SLA with Reordering of packets (as opposed to 'SLA' which is the 3-tier SLA with no Reordering). The second word of the label defines the client and the traffic class, for example 'Silver1' means Client1's Silver traffic. Finally, the last word of the label defines the metric we are plotting, for example 'Thru' means throughput. So, the label "SLAR/Silver1/Thru" identifies the color and line type of the curve representing the Client1's Silver throughput for the SLAR model. 


\subsection{Simulation Configuration}

\subsubsection{Topology}

We first start by describing the topology used in this scenario, also shown in Figure

15.

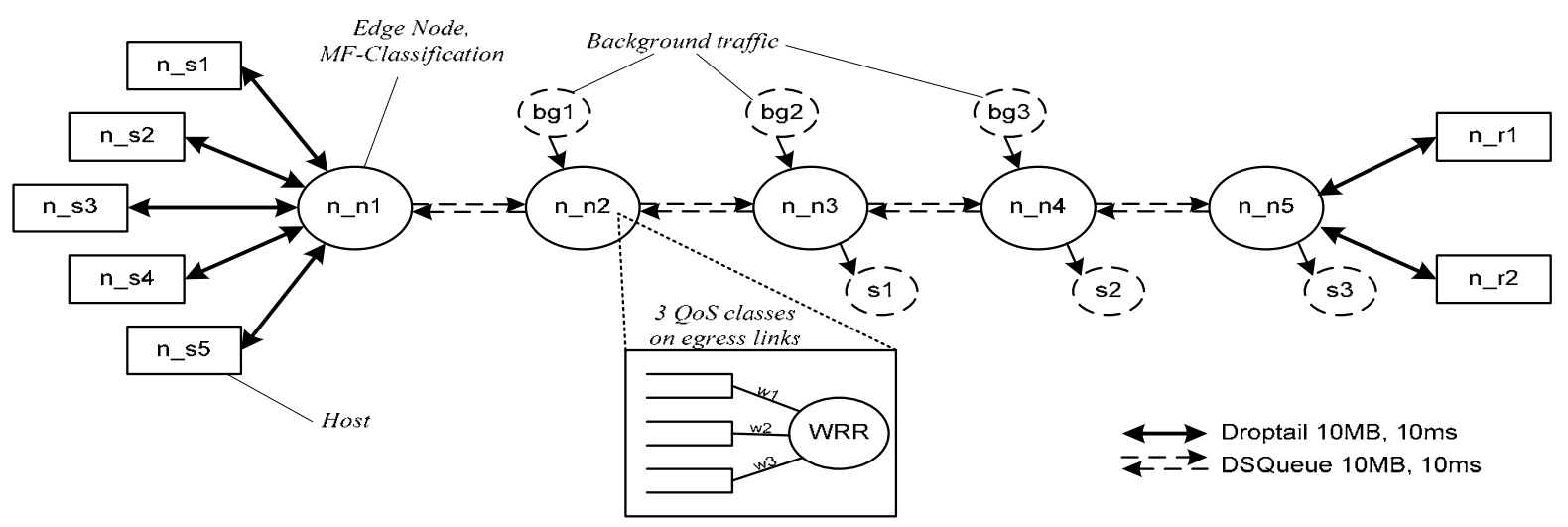

Figure 15 Network topology

The topology consists of 5 source hosts (or 5 clients) labeled n_s1 through n_s5 in the figure, 2 receiving hosts labeled $\mathrm{n} \_\mathrm{r} 1$ and $\mathrm{n} \_\mathrm{r} 2$, and 5 internal nodes. The number of nodes and hosts are chosen as such in order to keep the first scenario simple (i.e. relatively small number of nodes/hosts) and interesting (the higher the number of hops, the higher the effect of per hop queuing - in particular reordering - on QoS). 2 of the 5 clients (client1 and client2) will assume the 3-tier SLA and the other 3 clients (client3-client5) will act as background traffic sources each with a particular traffic class (Gold, Silver and Bronze respectively). The number of receiving hosts is chosen as 2 for traffic separation. 
The inner nodes, labeled n_n1 through n_n5, represent routers providing 3 classes of services. The first node, representing the edge node and labeled $n \_n 1$, classifies customer traffic (using a Multi-Field/MF classifier) and polices the offered traffic according to predefined rates (the customer negotiated rates). The inner nodes provide a BehaviorAggregate-like quality of service based on Gold, Silver and Bronze classes.

Hosts are connected to nodes via full-duplex links that assume a drop tail queuing (i.e. there is only one queue on egress going out on the link from each side), whereas nodes are connected via links with 3 egress queues and a guaranteed-rate scheduler. All links, including inter-node links, have a $10 \mathrm{MBps}$ capacity and a propagation delay of $10 \mathrm{~ms}$.

Inserted background traffic is of 2 types. Client3, Client 4 and Client5 (n_s3, n_s4 and n_s5, respectively) generate end-to-end background traffic (i.e from source to receive hosts), whereas bg1, bg2, and bg3 generate a single hop background traffic destined to s1, s2 and s3 respectively. The reason why bg1, bg2 and bg3 are added is to emulate converging traffic at each node (otherwise if the only background traffic is from source to destination, since the capacities of the links are the same, queuing would be observed in the first node only). Note that there is no need to inject background traffic at $n \_n 5$, being the last egress node of the network.

\subsubsection{Network setup}

In this scenario we assume all links have a capacity of $10 \mathrm{MBps}$ and a propagation delay of $10 \mathrm{~ms}$. The network capacity is partitioned so that the ratios of Gold, Silver and Bronze are 17, 38 and 45 respectively. These ratios were chosen to represent a provider's initial dimensioning of a network given that the highest priced traffic is Gold, then Silver, and then Bronze; thus a smaller ratio of the highest priced traffic to the lowest priced traffic. 
These ratios do not affect the behavior of the network, but they are assumed as hard reservations on each link of the network (schedulers are setup to guarantee these ratios on each link of the network).

Table 6 and Table 7 summarize the various parameters and values used in this scenario. In Table 6, the 'Rate' column describes the client traffic generation rate in Bytes per second, at a packet size defined in column 'Pkt Size'. The 'LB CBS' and 'LB CIR' represent the configured policing Leaky Bucket (LB) Committed Burst Size (CBS) in Bytes and CIR (Committed Informate Rate) in Bytes per second. The CBS and CIR apply to the first stage Meter (M) and second stage Policer (P) shown in Figure 9. Columns 'FS WRR' and 'SS WRR' represent the weights assigned to the Weighted Round Robin (WRR) hierarchy as shown in Figure 9. These weights will guarantee a certain fraction of the link capacity per traffic class per customer. And finally the 'GSR' column represents the Guaranteed Service Rate (GSR) provided by the WRR hierarchy per traffic class per customer. The GSR is calculated based on the WRR rate guarantee property, so for example, client1 gold GSR is:

$$
\begin{aligned}
\text { GSR client1 gold } & =[106 \times 269 \times 10 \mathrm{MBps}] / \\
& {\left[(106+399+495)^{\star}(269+65+100+257+309]\right.} \\
& =298990 \mathrm{Bps}
\end{aligned}
$$

Note that for bg1, bg2 and bg3 it is assumed that the traffic is policed on a different edge node (not shown in Figure 15) and thus the blank columns are not applicable. 
Table 6 Network Parameters, Scenario 1

\begin{tabular}{|l|l|l|l|l|l|l|l|l|l|}
\hline Client & Class & $\begin{array}{l}\text { Traffic- } \\
\text { type }\end{array}$ & $\begin{array}{l}\text { Rate } \\
\text { (Bps) }\end{array}$ & $\begin{array}{l}\text { Pkt Size } \\
\text { (B) }\end{array}$ & $\begin{array}{l}\text { LB CBS } \\
\text { (B) }\end{array}$ & $\begin{array}{l}\text { LB CIR } \\
\text { (Bps) }\end{array}$ & $\begin{array}{l}\text { FS } \\
\text { WRR }\end{array}$ & $\begin{array}{l}\text { SS } \\
\text { WRR }\end{array}$ & GSR \\
\hline Client1 & Gold & UDP CBR & 101457.5 & 500 & 5000 & 202915 & 106 & 269 & 298990 \\
\hline Client1 & Silver & UDP CBR & 814754 & 500 & 5000 & 763554 & 399 & 269 & 1125447 \\
\hline Client1 & Bronze & UDP CBR & 1050954 & 500 & 5000 & 948554 & 495 & 269 & 1396231 \\
\hline & & & & & & & & & \\
\hline Client2 & Gold & TCP FTP & Dynamic & $<1200$ & 5000 & 153600 & 25 & 65 & 227191 \\
\hline Client2 & Silver & TCP FTP & Dynamic & $<1200$ & 5000 & 153600 & 25 & 65 & 227191 \\
\hline Client2 & Bronze & TCP FTP & Dynamic & $<1200$ & 5000 & 153600 & 25 & 65 & 227191 \\
\hline & & & & & & & & & \\
\hline Client3 & Gold & UDP CBR & 713031.63 & 500 & 5000 & 713031 & 100 & 100 & 1048576 \\
\hline & & & & & & & & & \\
\hline Client4 & Silver & UDP CBR & 1834308.4 & 500 & 5000 & 1834308 & 100 & 257 & 2694840 \\
\hline & & & & & & & & & \\
\hline Client5 & Bronze & UDP CBR & 2204308.9 & 500 & 5000 & 2204308 & 100 & 309 & 3240099 \\
\hline & & & & & & & & & \\
\hline bg1 & Gold & UDP CBR & 534773.75 & 500 & & & & & \\
\hline bg1 & Silver & UDP CBR & 1375731.3 & 500 & & & & & \\
\hline bg1 & Bronze & UDP CBR & 1653231.6 & 500 & & & & & \\
\hline & & & & & & & & & \\
\hline bg2 & Gold & UDP CBR & 534773.75 & 500 & & & & & \\
\hline bg2 & Silver & UDP CBR & 1375731.3 & 500 & & & & & \\
\hline bg2 & Bronze & UDP CBR & 1653231.6 & 500 & & & & & \\
\hline & & & & & & & & & \\
\hline bg3 & Gold & UDP CBR & 534773.75 & 500 & & & & & \\
\hline bg3 & Silver & UDP CBR & 1375731.3 & 500 & & & & & \\
\hline bg3 & Bronze & UDP CBR & 1653231.6 & 500 & & & & & \\
\hline
\end{tabular}

Table 7 Nodes configuration, Scenario 1

\begin{tabular}{|l|l|l|l|l|l|l|}
\hline Node & Class & RED A & RED B & RED P & WRR & GSR \\
\hline n2n3 & Gold & inf & inf & 0 & 17 & 1782579 \\
\hline n2n3 & Silver & 10 & 20 & 0.5 & 38 & 3984588 \\
\hline n2n3 & Bronze & 20 & 30 & 0.7 & 45 & 4718592 \\
\hline & & & & & & \\
\hline n3n4 & Gold & inf & inf & 0 & 17 & 1782579 \\
\hline n3n4 & Silver & 10 & 20 & 0.5 & 38 & 3984588 \\
\hline n3n4 & Bronze & 20 & 30 & 0.7 & 45 & 4718592 \\
\hline & & & & & & \\
\hline n4n5 & Gold & inf & inf & 0 & 17 & 1782579 \\
\hline n4n5 & Silver & 10 & 20 & 0.5 & 38 & 3984588 \\
\hline n4n5 & Bronze & 20 & 30 & 0.7 & 45 & 4718592 \\
\hline
\end{tabular}


Table 7 above shows the egress configuration of the buffer management (Random Early Discard - RED) and scheduler on each node in the topology. RED [82] allows packets to pass when the average queue size is less than a configured threshold A, drops packets with probability $\mathrm{P}$ when the average queue size is in the region $(\mathrm{AB})$, and drops all packets when the average queue size is larger than a configured threshold B. 'RED A', 'RED B' and 'RED P' columns represent the RED parameters being the two threshold points A and B and the drop probability P, respectively. The RED buffer management and the queuing/scheduling in the inner nodes provide differentiated services based on traffic class aggregates and respective configuration. Note that since all links are full-duplex links and nodes require reverse direction configuration, the reverse direction node configuration values (WRR weights and RED parameters) are the same as the forward direction values (and thus not shown in the table).

The table also shows that except for client 2, all traffic types are UDP CBR (Constant Bit Rate). As an initial scenario, CBR was chosen to show more easily the concept of automatically upgrading lower class packets to fill up higher class gaps, and to highlight some of the findings and control parameters. In later scenarios we will use different (bursty) traffic sources. TCP is used for client 2, in order to show some of the key points related to TCP.

In the following sections we describe the various aspects of the SLA in comparison with the DiffServ and the SLA with reordering models. 


\subsection{Automatic upgrades}

We begin by showing how the components of Figure 9 automatically upgrade the lower class packets to fill up the gaps in the upper classes for the SLAR (SLA with Reordering).

From Table 6, Client1's policed Gold, Silver and Bronze rates are $200 \mathrm{KBps}$, $\sim 760 \mathrm{KBps}$, and $\sim 950 \mathrm{KBps}$, respectively. In this first experiment, we vary the Gold ingress rate while keeping the Silver and Bronze ingress rates at $\sim 815 \mathrm{KBps}$, and $\sim 1050 \mathrm{KBps}$, respectively. We expect the Silver packets to be upgraded to Gold to fill up any Gold bandwidth gap, and the Bronze packets to be upgraded to Silver to fill up the remaining Silver gap.

Figure 16 shows client1 Silver and Bronze throughput (upgrades included) measured at ingress edge node $n \_n 1$, and Figure 17 shows the respective upgraded rates when varying the Gold traffic rate (i.e. CBR1) from 0 to $243,498 \mathrm{Bps}$ at a 20,291 Bps increments. Notice that confidence intervals values - not observable in this figure - are negligible compared to the rates (less than $1 \%)$. 


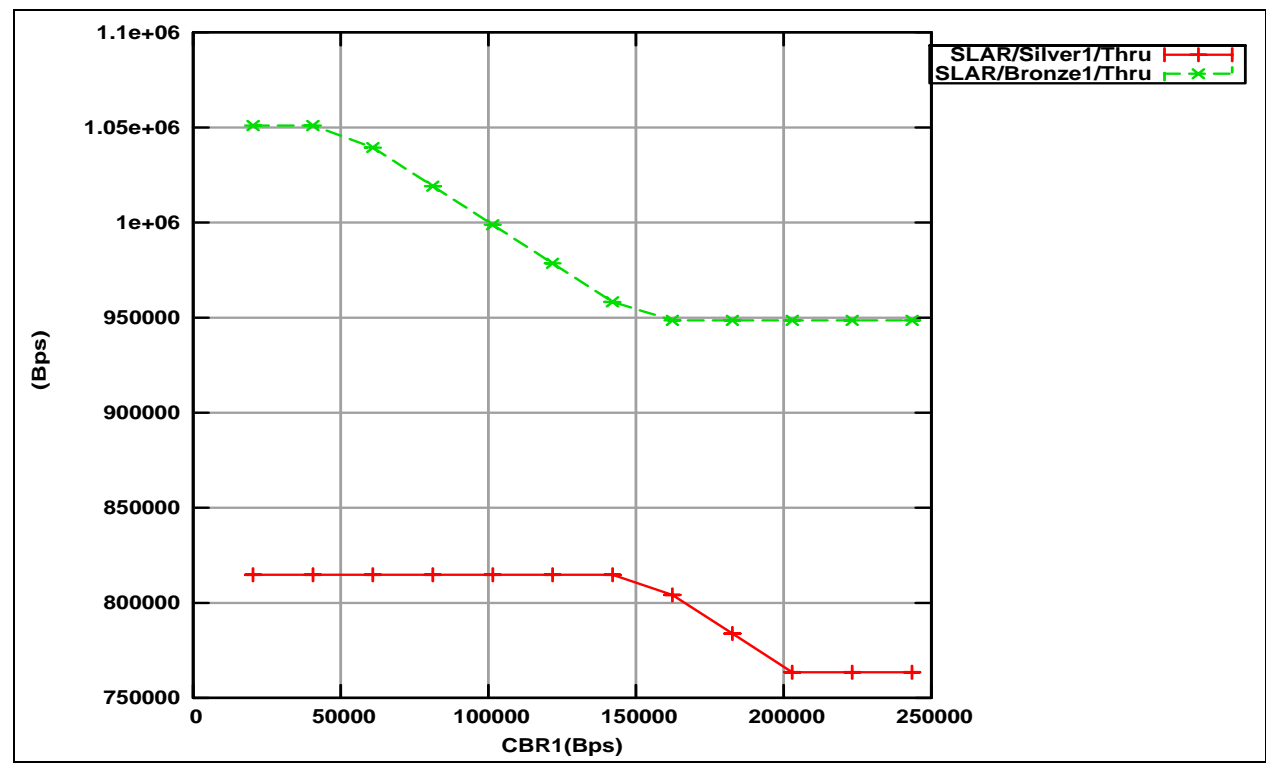

Figure 16 Silver and Bronze Throughput versus Gold rate

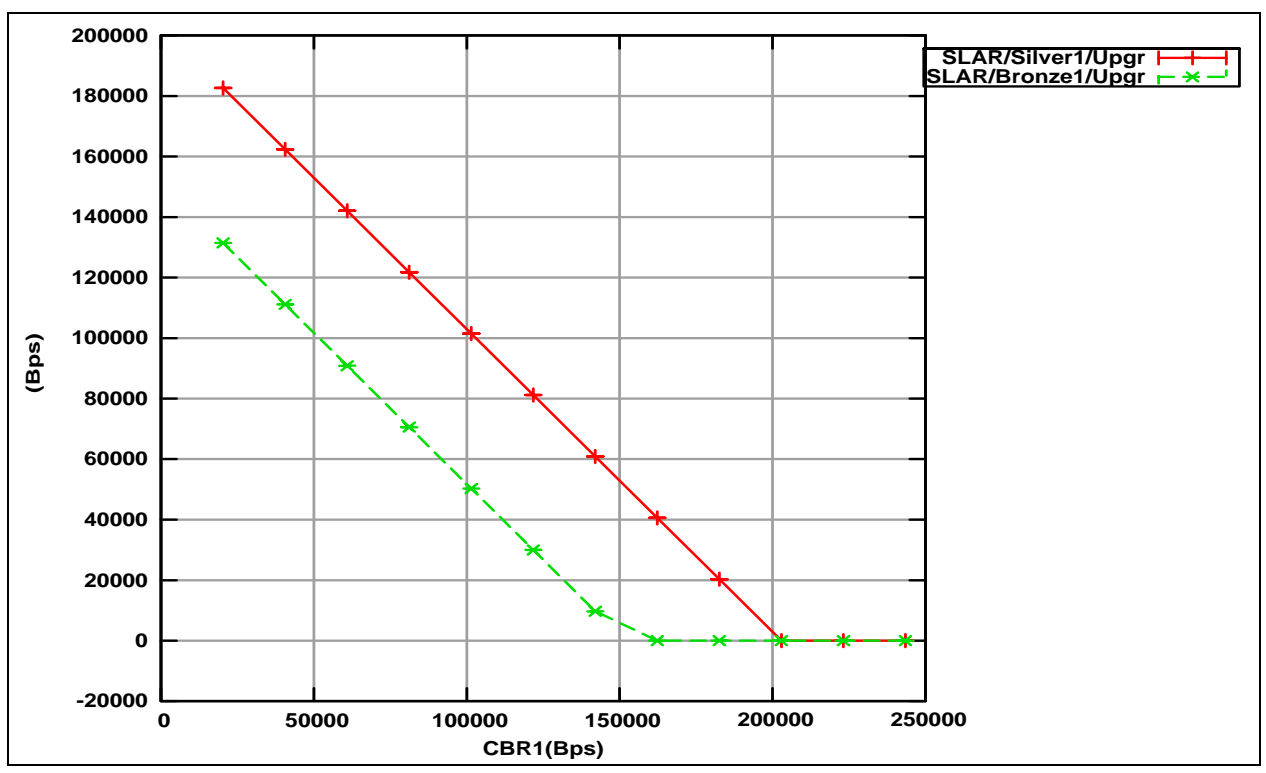

Figure 17 Silver and Bronze upgrade rates versus Gold rate

Referring to Figure 16 and Figure 17, when Client1's Gold traffic is at 20 KBps, $\sim 182 \mathrm{KBps}$ Silver traffic is upgraded to fill the $\sim 182 \mathrm{KBps}(202915-20 \mathrm{~K})$ Gold gap, and the remaining $\sim 632 \mathrm{KBps}(814754-182 \mathrm{~K})$ are found conformant to the policed $763554 \mathrm{CIR}$. The 
$632 \mathrm{KBps}$ Silver traffic create a gap of $\sim 131 \mathrm{KBps}$ (763554-632K) in Silver which the Bronze traffic takes advantage of. The remaining $\sim 919 \mathrm{~K}$ of Bronze traffic are found conformant to the policed 948554 CIR. So the total forwarded rates for Silver and Bronze, when the Gold is at $\sim 20 \mathrm{~K}$, are $\sim 814 \mathrm{~K}(182 \mathrm{~K}$ upgraded $+632 \mathrm{~K})$ and $1050 \mathrm{~K}(131 \mathrm{~K}$ upgraded $+919 \mathrm{~K})$ respectively.

Similarly, Figure 18 shows client1 Gold and Bronze throughput (upgrades included) at ingress (ingress edge node $n \_n 1$ ) and Figure 19 shows the respective upgraded rates when varying the Silver traffic rate (i.e. CBR2) from 0 to 916264 Bps at a 76,355 increments (Client1 Silver traffic is policed at $760 \mathrm{KBps})$.

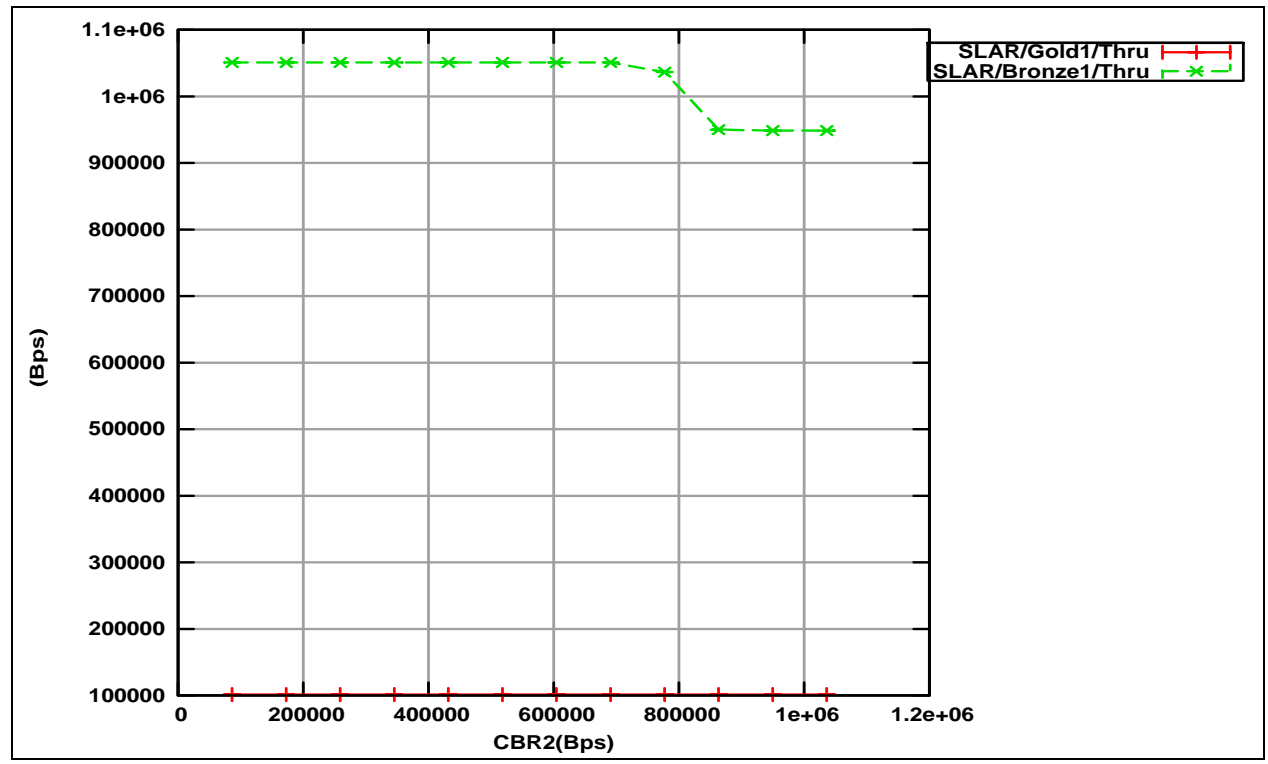

Figure 18 Gold and Bronze Throughput versus Silver rate 


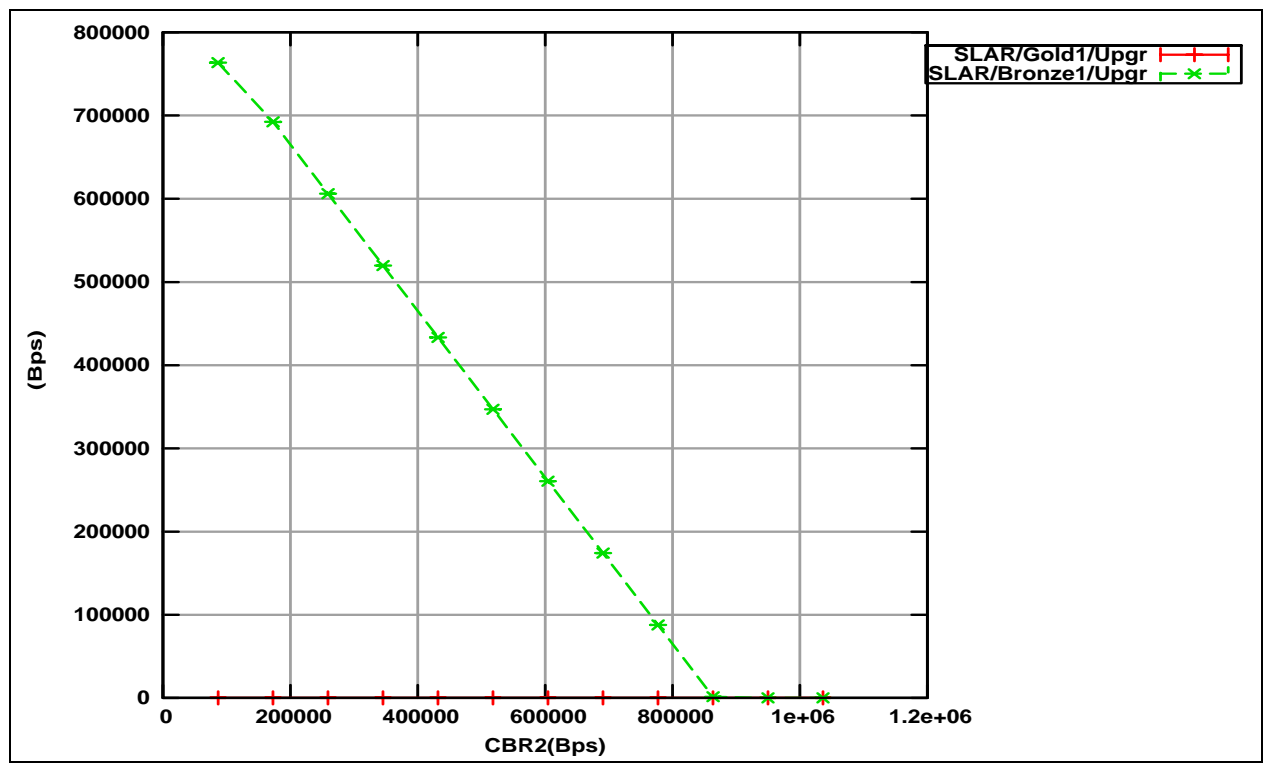

Figure 19 Gold and Bronze upgrade rates versus Silver rate

Notice in the figures above, the Gold rate is not affected by the Silver rate. However, as more Silver rate is added to the network the less the Bronze traffic is upgraded (more Silver traffic means thinner Silver gap). The Bronze throughput starts to get affected when there is not enough of a Silver gap to accommodate the excess Bronze traffic of (1050954 $948554)=\sim 100 \mathrm{KBps}$. When the Silver rate is at $760 \mathrm{KBps}$, and $\sim 100 \mathrm{~K}$ Silver traffic is upgraded to fill the Gold 100KBps gap, a Silver gap of $763554-660 \mathrm{~K}=\sim 100 \mathrm{KBps}$ is created. If more than $760 \mathrm{KBps}$ Silver traffic is sent by the customer, the excess Bronze traffic can no longer be accommodated and will be dropped.

Even though the Silver and Bronze classes were using the gaps in the upper class to achieve higher throughput at the ingress node, the observed goodput at the receiver (node n_r1), shown in Figure 20, does not reflect that they got better service. Notice how the Silver traffic got much less goodput than the expected full rate $750 \mathrm{KBps}$. This is better observed when we plot the throughput versus the goodput. 


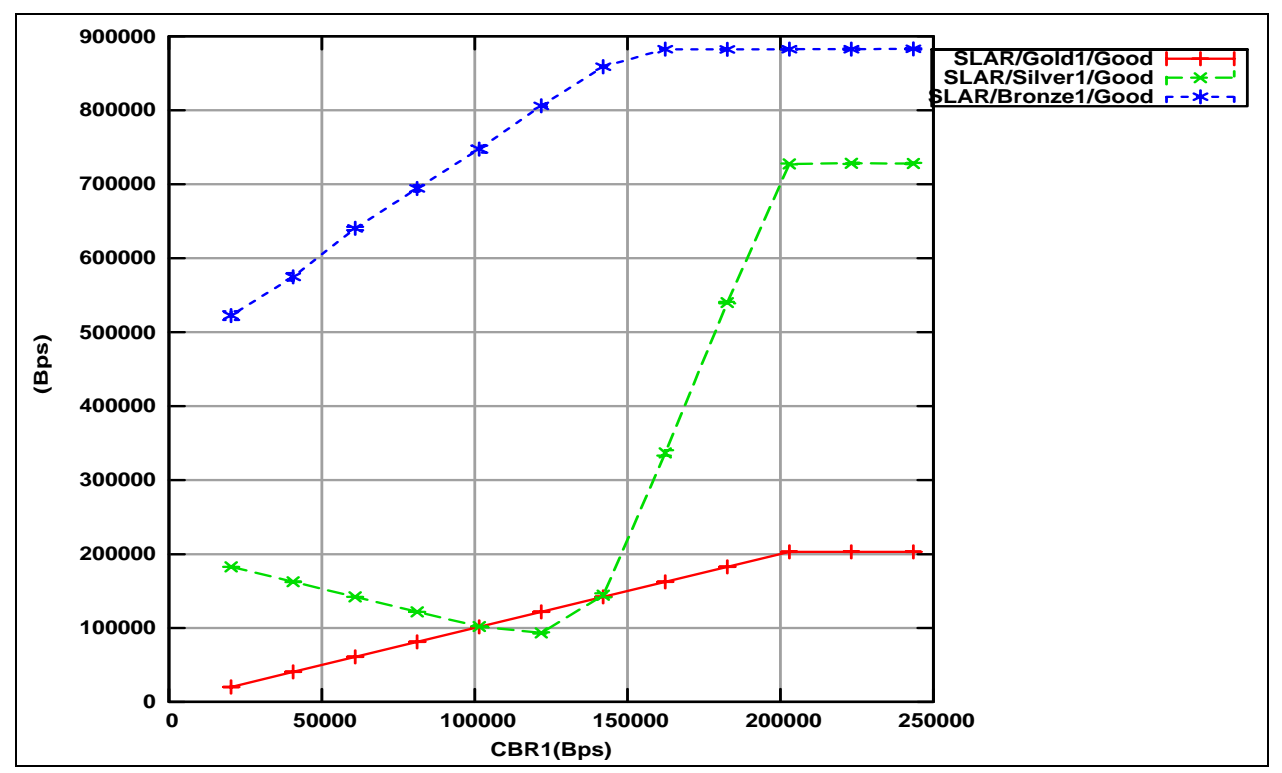

Figure 20 Client1 Goodput Versus Gold rate

Figure 21 shows the Silver and Bronze goodput at the receiver versus the throughput at the ingress node, as CBR1 increases from 0 to 25,000 Bps. The figure clearly shows that there were major losses for the Silver and Bronze classes when the Gold traffic is relatively low.

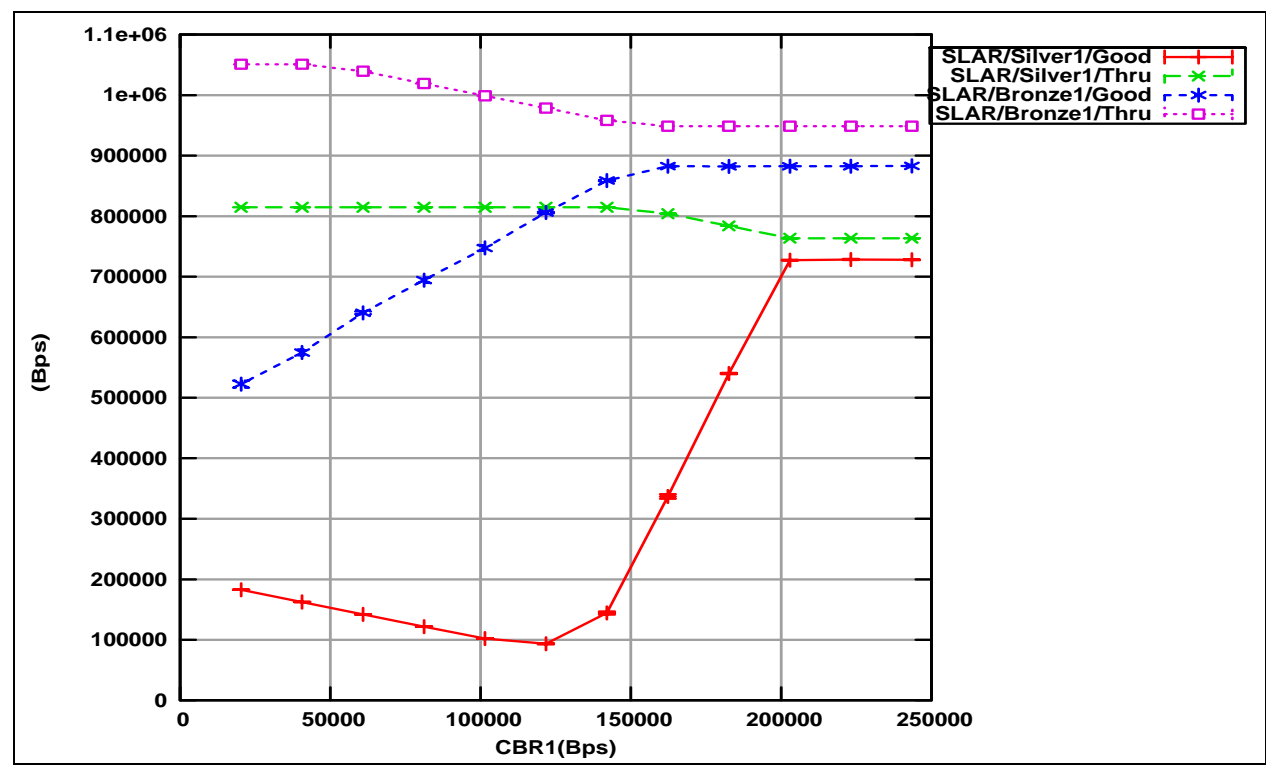

Figure 21 Client1 Goodput and Throughput Versus Gold rate 
This fact is explained by the amount of reordering the Silver and Bronze traffic observe when the Gold traffic is relatively low. Here, the reordered packets at the receiver are treated as losses since the receiver doesn't keep a replay buffer. Since the network Silver and Bronze queues are often non-empty, whenever a Silver or Bronze packet is upgraded it will be serviced from end-to-end as a higher class packet bypassing, with a high probability, previous packets from the same flow (higher class is better service). The higher the nonsequential (non-back-to-back) upgrades are, the higher the probability of reordering. However, when a burst is upgraded, packets within the burst will arrive in sequence, but may arrive out of sequence relative to packets transmitted before the burst (the amount of reordering due to burst upgrade could be lower than random upgrade). In general, the highest amount of upgrades is observed when the Gold traffic is at its minimum; that is why the highest losses occur when the Gold traffic is relatively low. When the Gold traffic rate is relatively high (fills most of the Gold negotiated rate), the excess Silver traffic has less chance of being upgraded and will be found non-conformant to the Silver negotiated rate (thus dropped). Moreover, since the network is relatively loaded, when the Gold rate is high the Silver queues in the network fill up and will be controlled by the RED management algorithm which will drop packets from the Silver aggregate. Figure 22 shows the number of reordered packets as the Gold traffic is increased. The figure shows that after a certain point $(\sim 125000 \mathrm{Bps})$ the amount of Silver reordered packets drastically decreases since the Silver traffic has a smaller gap in the Gold negotiated rate and less packets are being upgraded. Notice how the Silver rate picks up as indicated in Figure 21 at exactly the same point. This is due to the fact that the Silver traffic is mostly being forwarded as Silver (i.e. not upgraded 
or not reordered and thus not dropped): For a particular throughput, lower reordering means higher goodput.

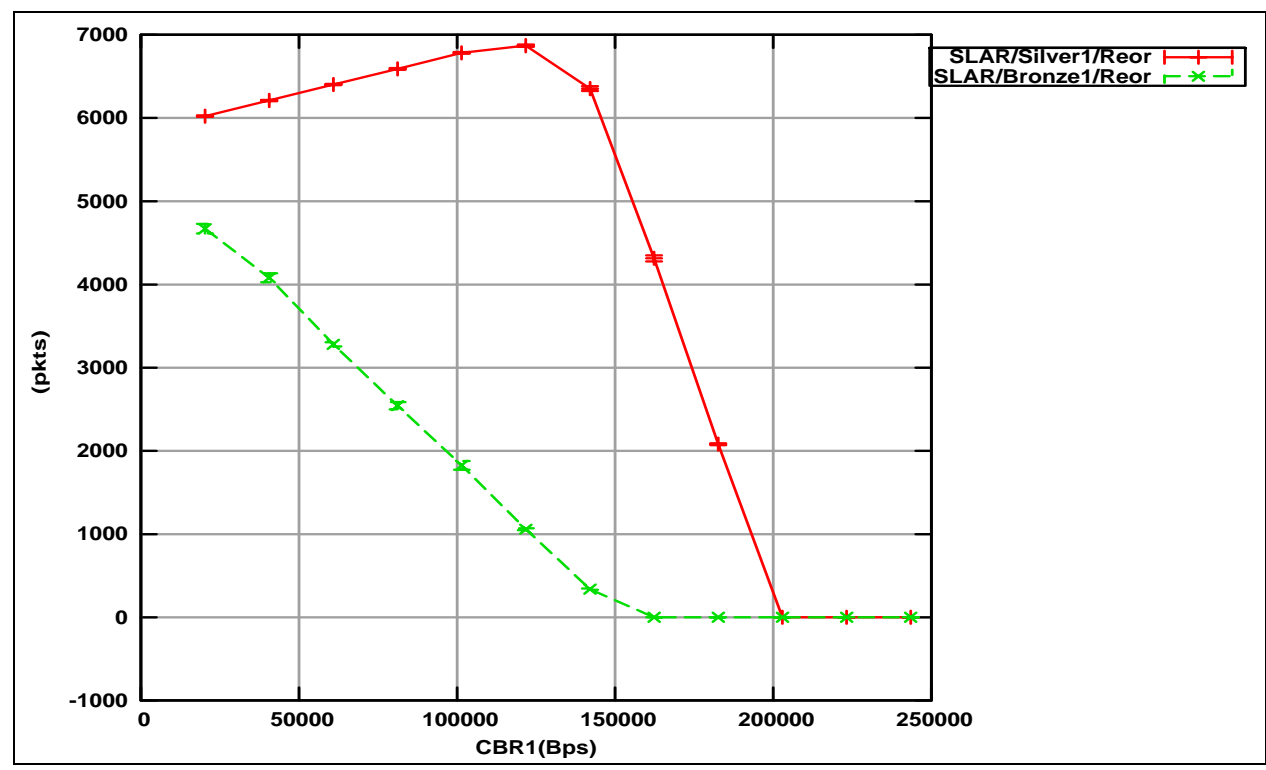

Figure 22 Client1 Reordered packets Versus Gold rate

In Figure 18, we varied Client 1's Silver traffic while keeping the Gold unvaried in order to show how the Bronze traffic (ingressing the network at a rate of 1050MBps) takes advantage of the Silver gap thus passing more traffic at the ingress (better throughput). The Bronze rate maxed out when there was no more room for Bronze upgrades (Bronze is policed at $\sim 950 \mathrm{KBps}$ ). Moreover, since the Gold rate is set at $\sim 100 \mathrm{KBps}$, the Silver rate maxes out at $\sim 850 \mathrm{KBps}$ (policed at $\sim 750 \mathrm{KBps}+100 \mathrm{Kbps}$ upgraded to Gold). However, for the same reasons mentioned above i.e. packet reordering, the Bronze goodput observed at the receiver shows that the Bronze traffic did not get any better service even though it got better throughput (Figure 23). The Silver traffic goodput was at most 100KBps (the upgrade rate) 
when the Silver ingress rate was higher than $500 \mathrm{KBps}$ since all non-upgraded packets got reordered. Notice the amount of reordering for both Silver and Bronze (Figure 24).

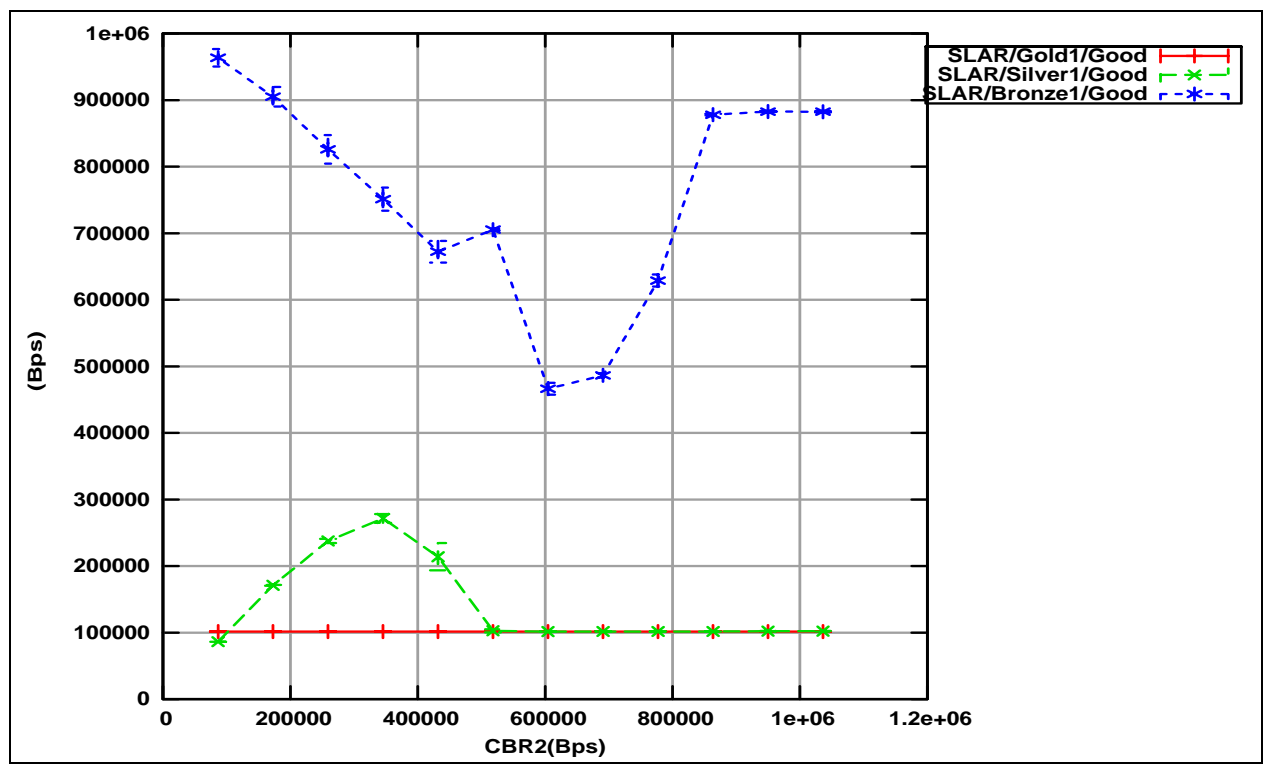

Figure 23 Gold, Silver and Bronze Goodput Versus Silver rate

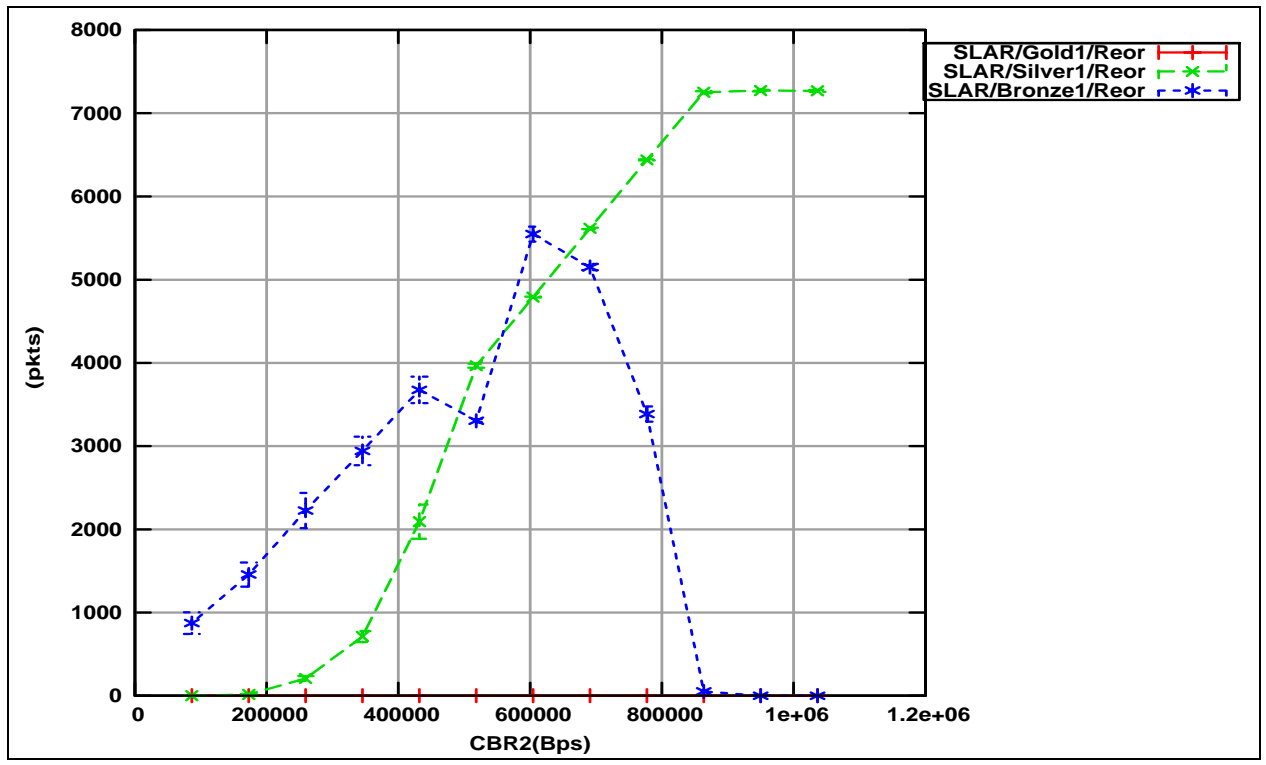

Figure 24 Gold, Silver and Bronze Reordering Versus Silver rate 
To summarize this section's findings, we have shown that the ingress QoS components of Figure 9 automatically upgrade lower class packets to fill up gaps in the upper classes. However, since the ingress components can not take care of the reordering problem that occurs in the network, a reordering solution is needed to achieve higher goodputs, especially for receivers that do not buffer out of order packets.

\subsection{Applying the reordering solution}

In the previous section, we showed that upgrading packets on the ingress improves the throughput of the sources but the goodput at the receiver is much worse than expected. This fact was due to the reordering of packets which proved very harmful. In section 5.4, we talked about a reordering solution that prevents packets from being reordered due to QoS upgrades. Applying this solution and repeating the same experiments of the previous chapter shows that upgrading packets without reordering could be of great benefit for clients. Finally, comparing the 3-tier SLA with the DiffServ behavior in case of no upgrades demonstrates the benefit of such an SLA.

\subsubsection{QoS Performance}

\subsubsection{Throughput and Goodput}

First we show in Figure 25 and Figure 26 the behavior at the ingress by displaying Client1's Silver and Bronze throughput versus the Gold rate of each of the 3 models: Diffserv (DS), SLA without reordering solution (SLAR), and SLA with the reordering solution (SLA). 


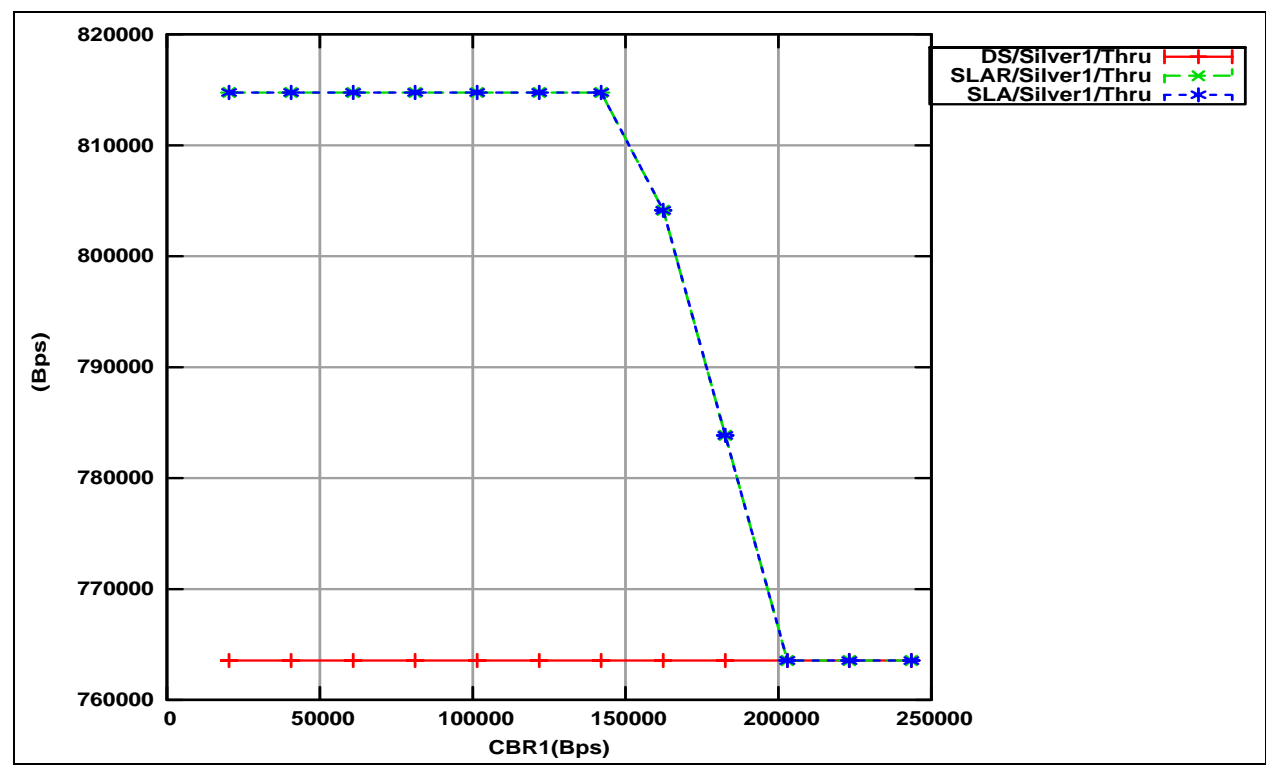

Figure 25 DS, SLAR and SLA Silver Throughput Versus Gold rate

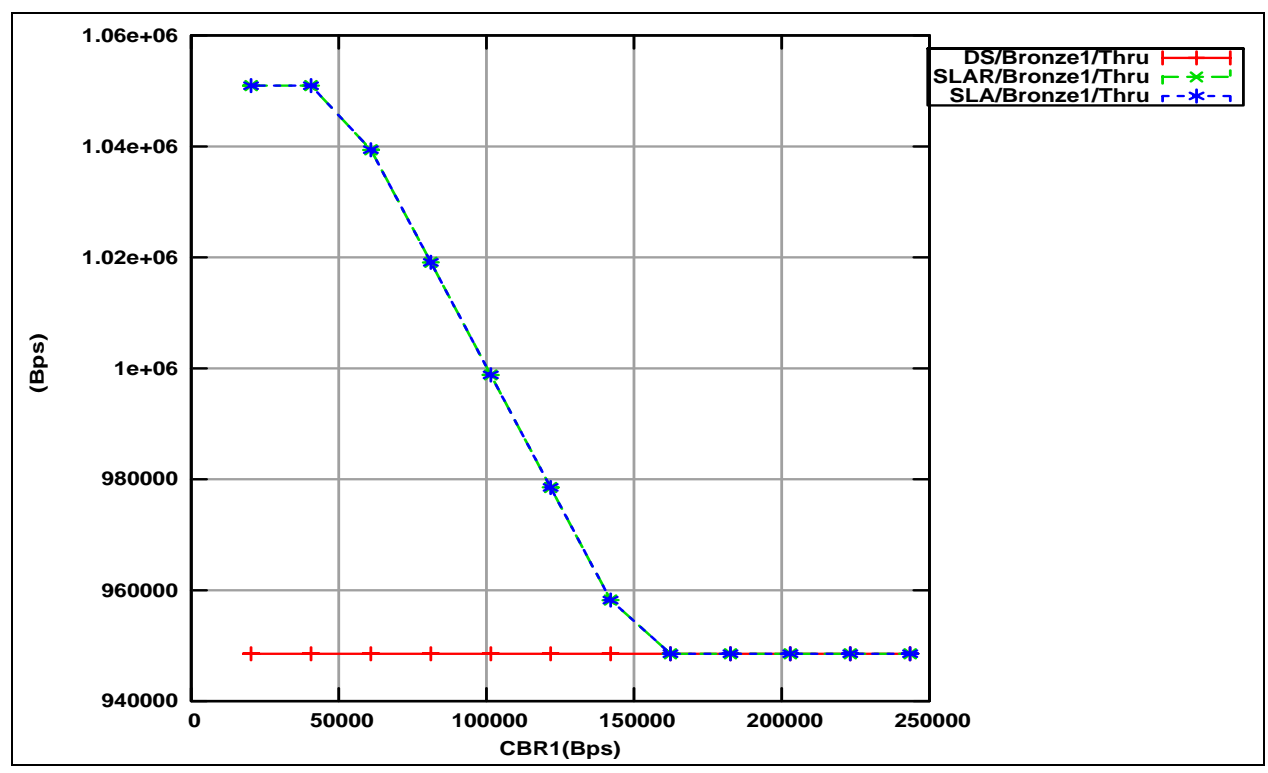

Figure 26 DS, SLAR, SLA Bronze Throughput Versus Gold rate

The figures above show that both the SLAR and the SLA models allowed more Silver and Bronze traffic at the ingress to fill up the Gold and Silver gapes respectively. However, since the DS policy was to drop the excess ingress traffic, only the policed Silver 
( $765 \mathrm{KBps})$ and Bronze ( $\sim 950 \mathrm{KBps})$ rates were allowed into the network, regardless of whether the Gold and Silver reserved bandwidths were fully used or not.

Figure 27 and Figure 28 show the difference in terms of Client1's Silver and Bronze goodput of the DS, SLAR and SLA models when the Gold rate is varied. As explained earlier, the SLAR behavior of lower goodput is due to packet reordering in the network. On the other hand, the DS model shows no significant improvement since it is tightly policed at the ingress of the network i.e. all excess traffic is dropped. Finally, the SLA model shows that the customer benefits not only from a higher throughput, or accepted traffic at the ingress, but also from a higher goodput at the receiver. The SLA gives the client the ability to fully benefit from his higher classes' reserved bandwidths without any reordering in the network.

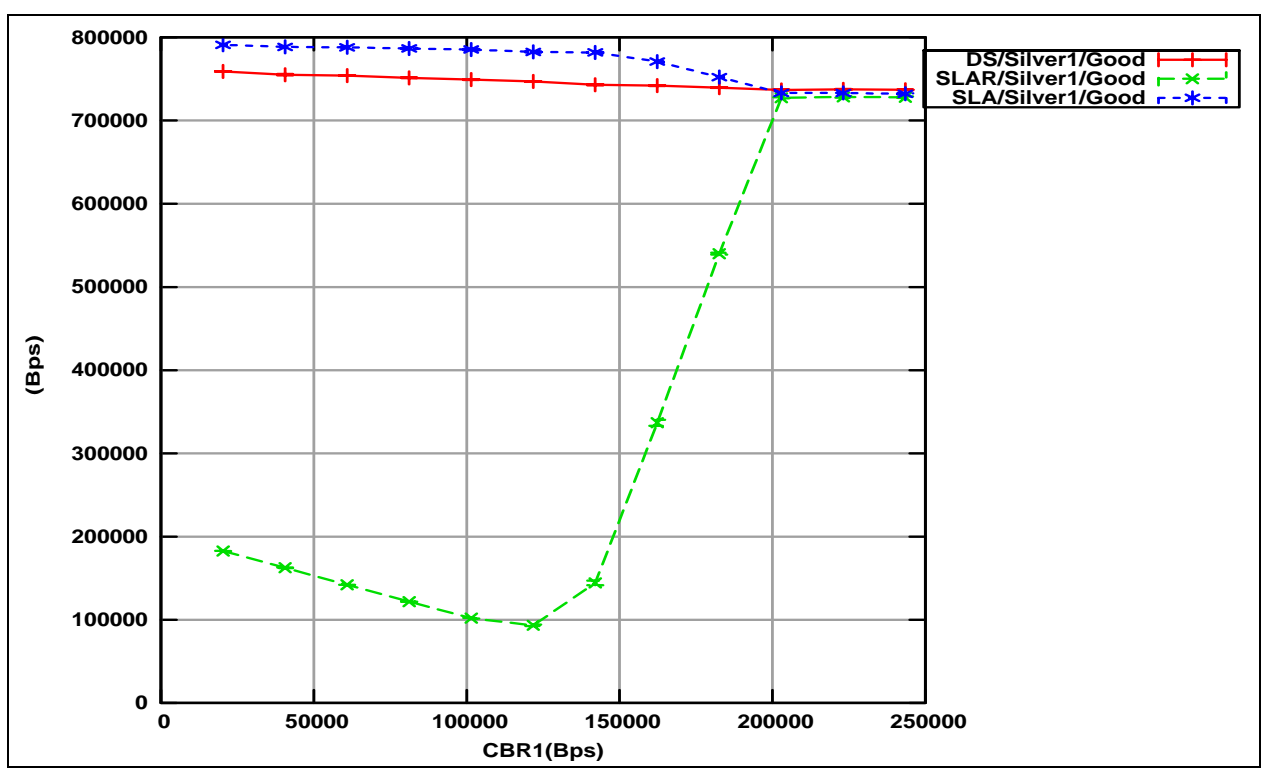

Figure 27 DS, SLAR, SLA Silver goodput Versus Gold rate 


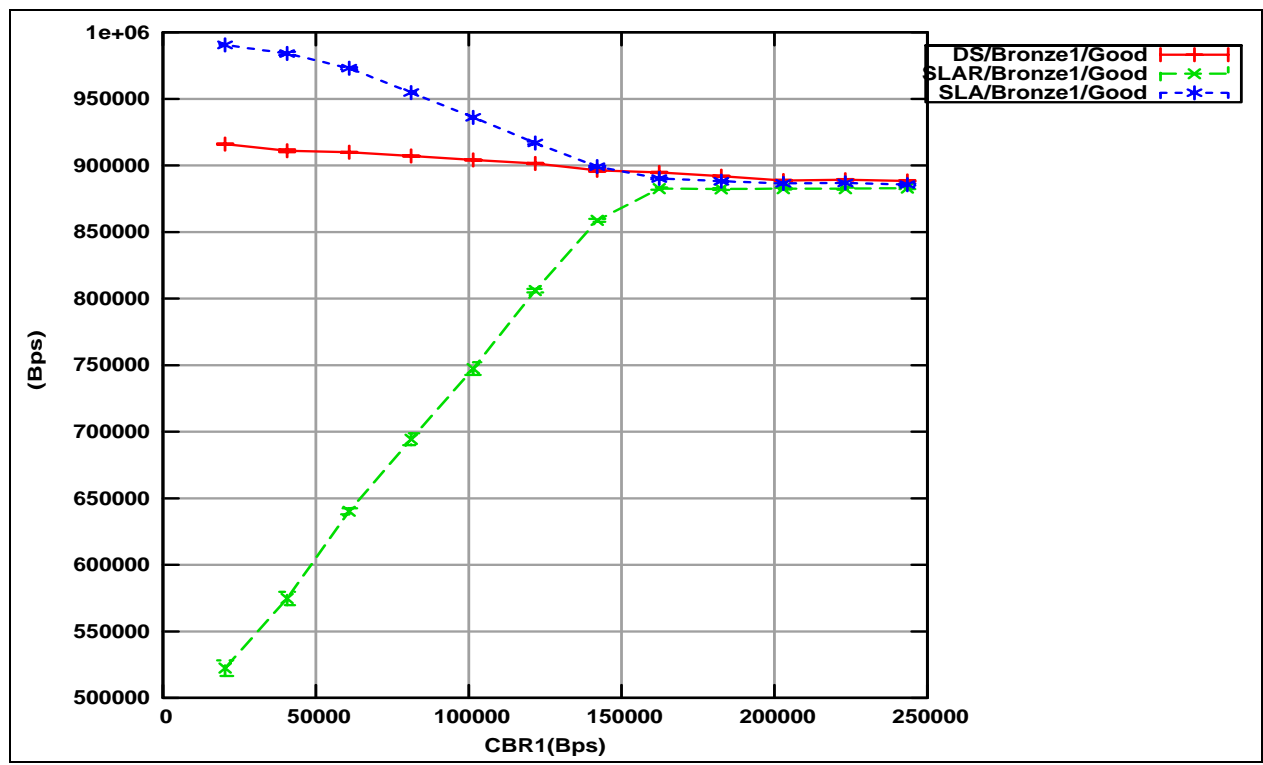

Figure 28 DS, SLAR, SLA Bronze goodput Versus Gold rate

Notice in the above figures that the DS Silver and Bronze goodputs are slightly decreasing. This is explained by the fact that as more Gold traffic is pushed into the network, the lower classes will be impacted. This behavior is typical in diffserv networks were higher classes are differentiated at the expense of lower classes.

In Figure 29 we display the goodput of the 3 models when varying Client1's Silver rate. Notice that the SLA goodput is much better than the DS model due to upgrades, and the SLAR goodput is much worse than the DS model due to reordering. 


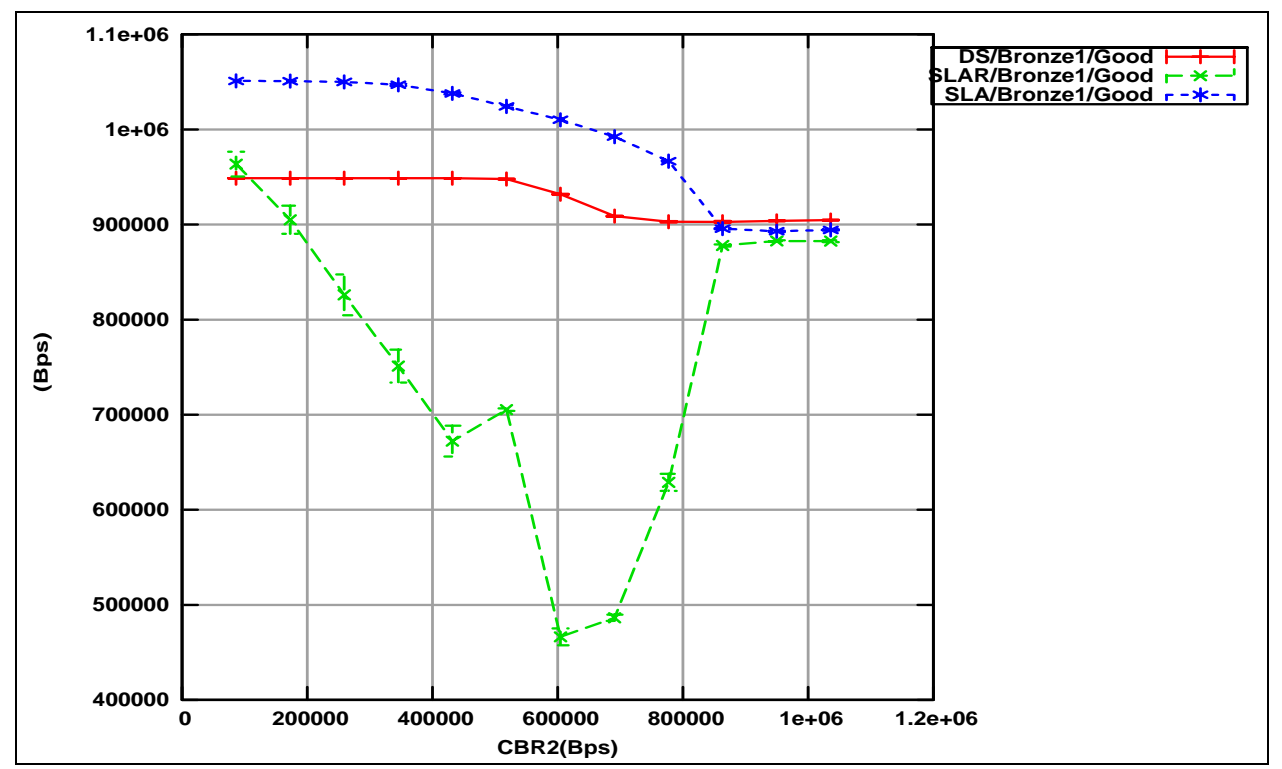

Figure 29 DS, SLAR, SLA Bronze goodput Versus Silver rate

In the following figures, we show the network behavior in terms of Client1's throughput and goodput for each of the models. The DS model, refer to Figure 30, shows that as the Gold rate increases both the Silver and Bronze goodputs are affected, a typical diffserv behavior where higher classes are given preferential treatment at the expense of the lower classes. Notice that the throughput at the ingress of the Silver and Bonze traffic does not change as the Gold rate increases, since the excess Silver and Bronze traffic are dropped. As for the SLAR model, as explained before, even though the Silver and Bronze traffic observe a higher throughput at the ingress, the goodput is highly affected by the reordered packets in the network. Compared to the DS model, it seems that the SLAR is not a good service to offer to customers. 


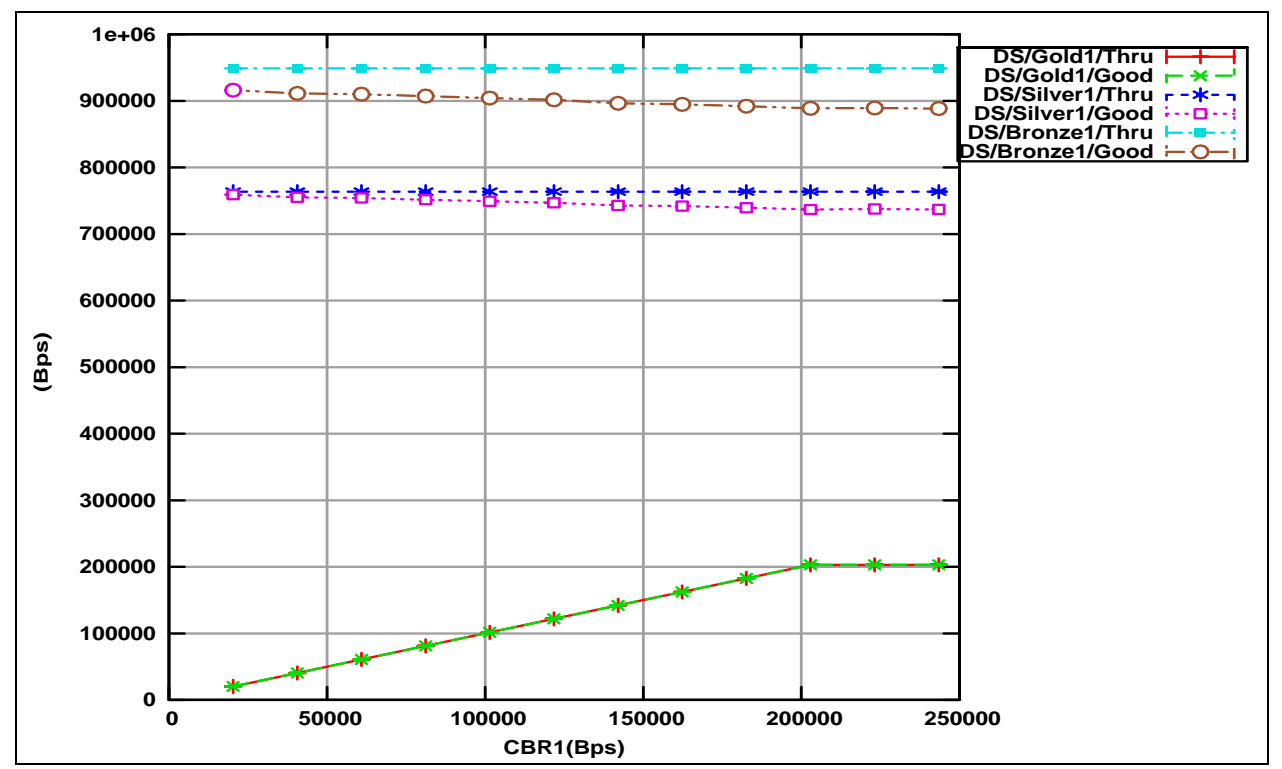

Figure 30 DS Throughput and Goodput Versus Gold rate

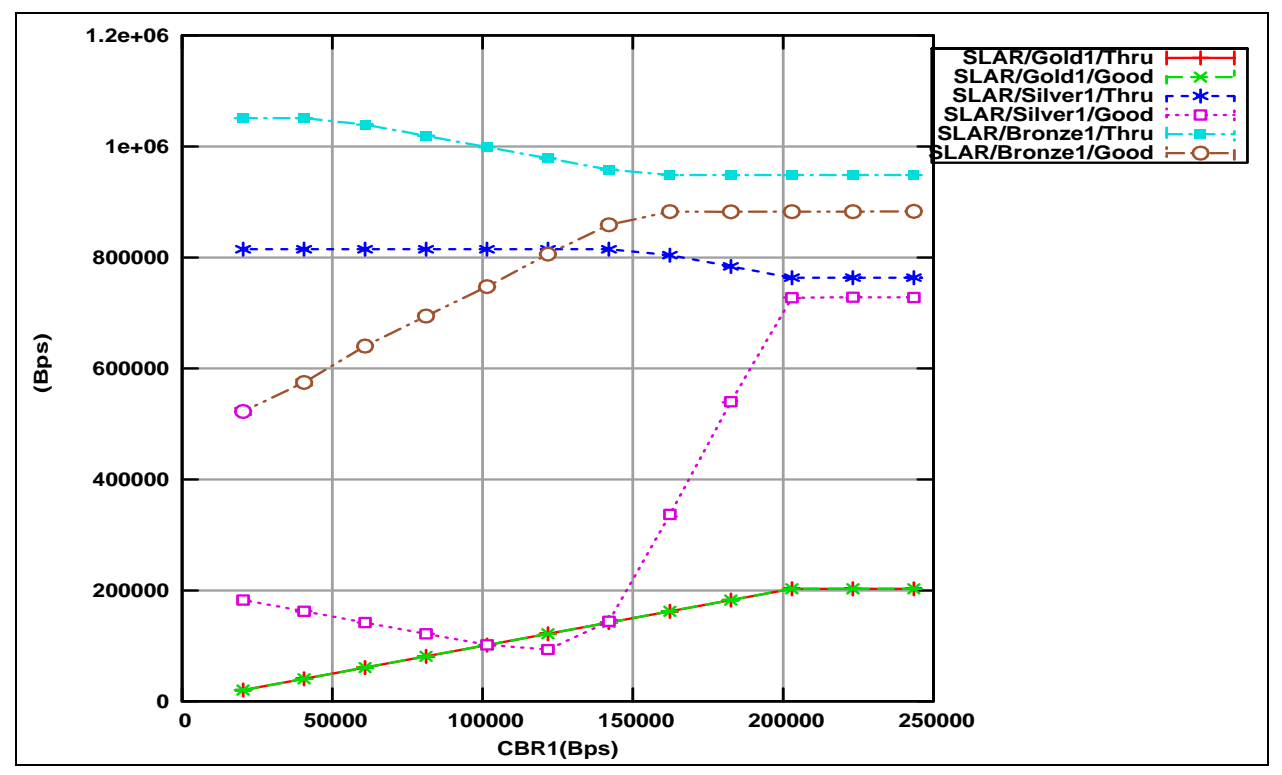

Figure 31 SLAR Throughput and Goodput Versus Gold rate

Finally, the SLA model shows a significant improvement over the other two models since it clearly shows the improved service the customer gets when there is room to upgrade Silver and Bronze packets. The differences in goodput of the three models are highlighted in Figure 27 and Figure 28. 


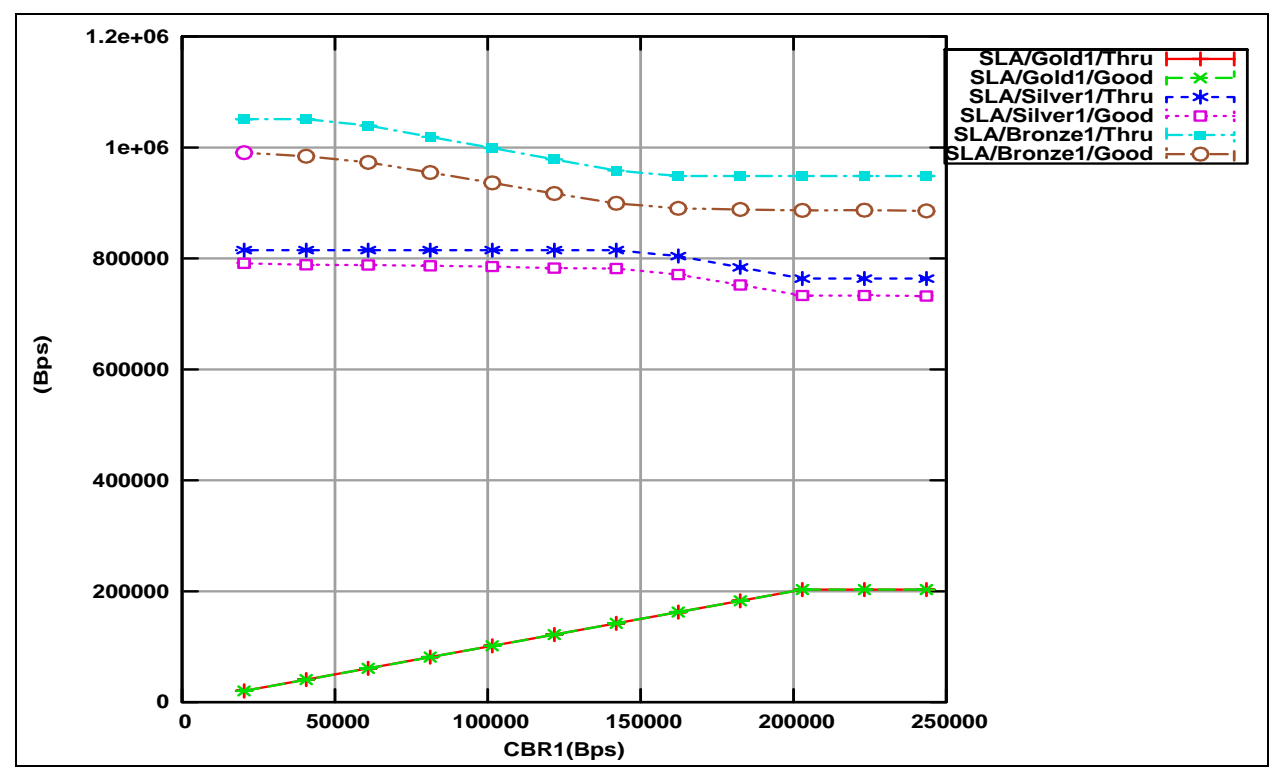

Figure 32 SLA Throughput and Goodput Versus Gold rate

Notice the existence of a gap between the throughput and goodput of the Silver and Bronze flows in Figure 32. This is due partially to aggregate losses due to network congestion, and partially to ingress policing when the Gold rates are high enough not to allow upgrades, as we will see in the next section.

\subsubsection{Packet Loss}

Figure 33 shows the SLA model's total losses (due to ingress policing and network congestion) in packets at the receive side (the losses displayed are absolute i.e. non cumulative). The losses increase as the Gold rate is increased; As the Gold rate narrows the Gold bandwidth gap, the Silver and Bronze packets find less and less room to be upgraded, and the losses are more severe; this is due to ingress policing where excess traffic is no longer upgraded but rather dropped. Notice that there are some Gold losses due to ingress policing, since the Gold traffic is policed at $\sim 200 \mathrm{KBps}$. Figure 34 shows the loss rate due to 
ingress policing only. Again, as the Gold traffic is increased, there is smaller room for upgrades and both the Silver and Bronze flows start observing packet drops.

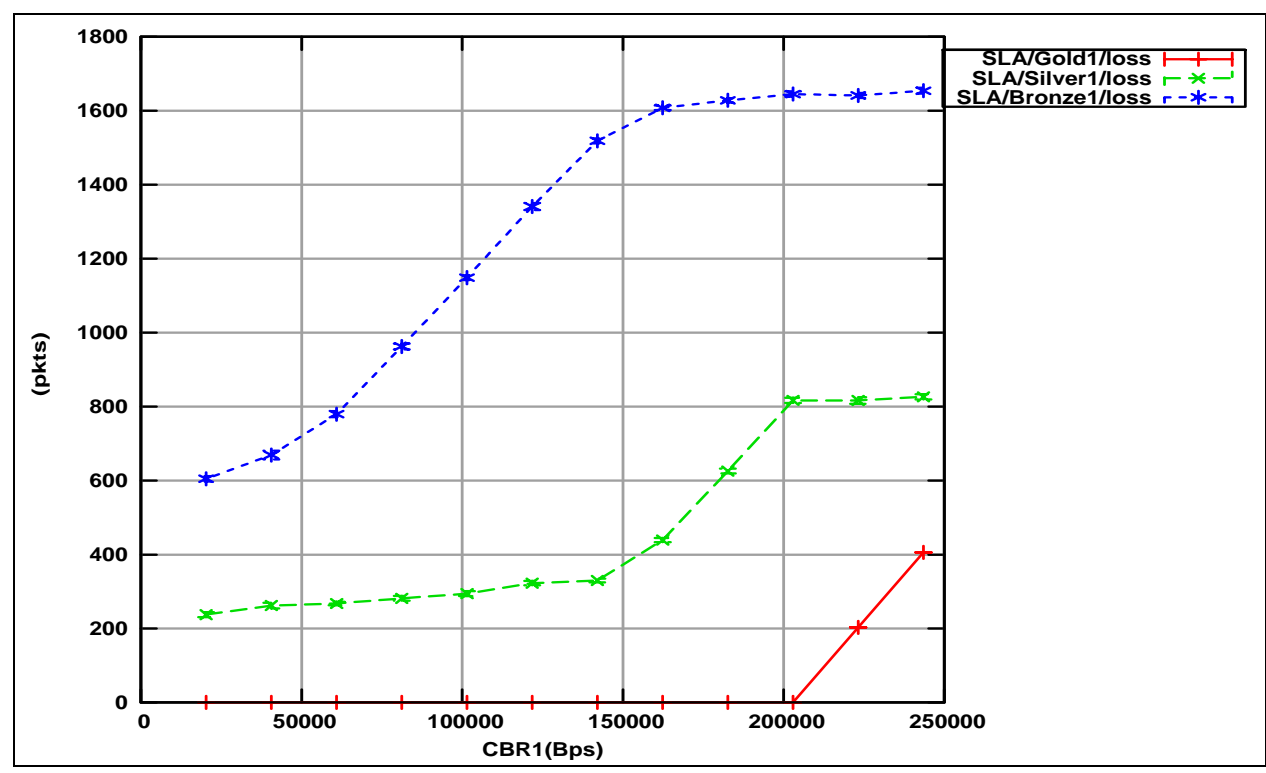

Figure 33 SLA losses Versus Gold rate

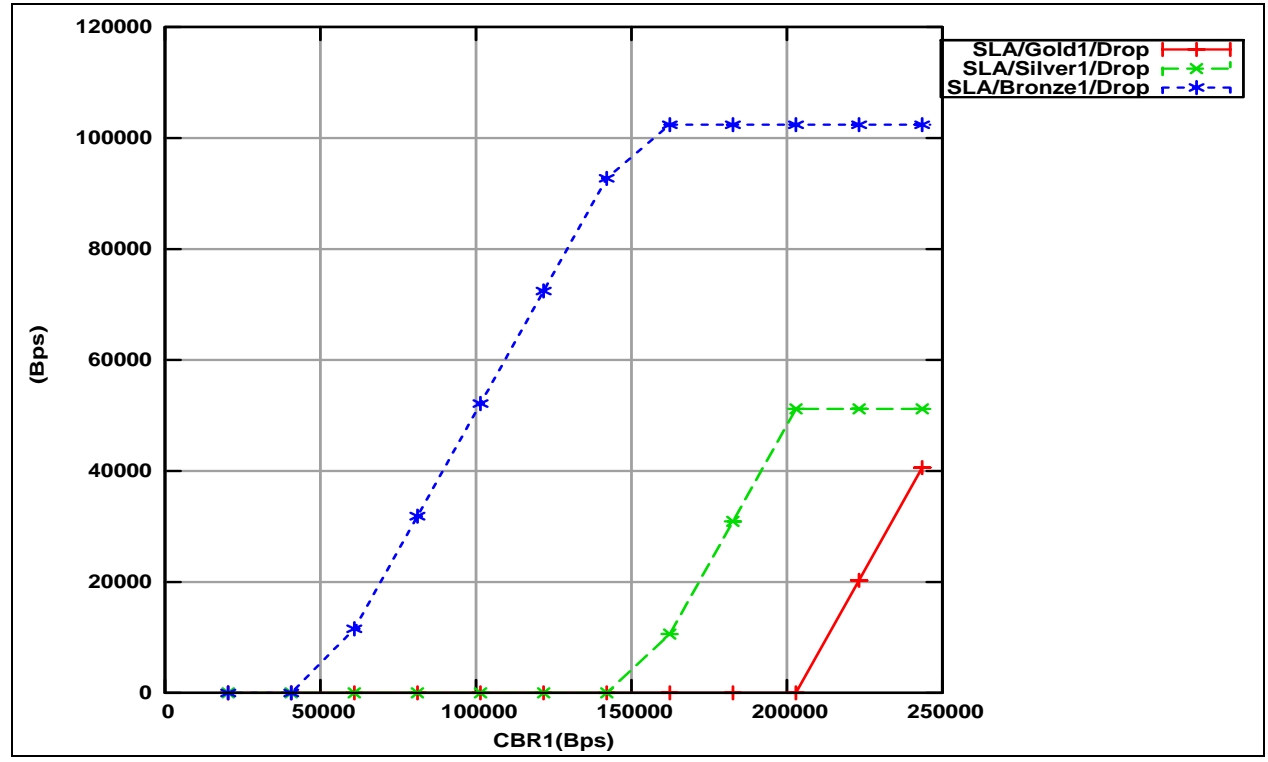

Figure 34 SLA ingress policing drops Versus Gold rate 
Figure 35 shows Client1's Silver traffic packet loss comparison of the 3 models. The DS model shows an almost constant Silver drop due mainly to ingress policing. The Silver losses are aggravated when the Gold rate is increased (higher class differentiation). The SLAR model observed heavy reordering, as shown previously, which are considered losses at the receiver. And finally, the SLA model observed the least amount of reordering due to class upgrades and no reordering in the network. This further demonstrates the advantage of the 3tier SLA model.

Figure 36 shows the ingress drop rates of the various models. Notice that the SLAR and the SLA have similar behavior on the network edge, the difference being in the reordering of packets inside the network.

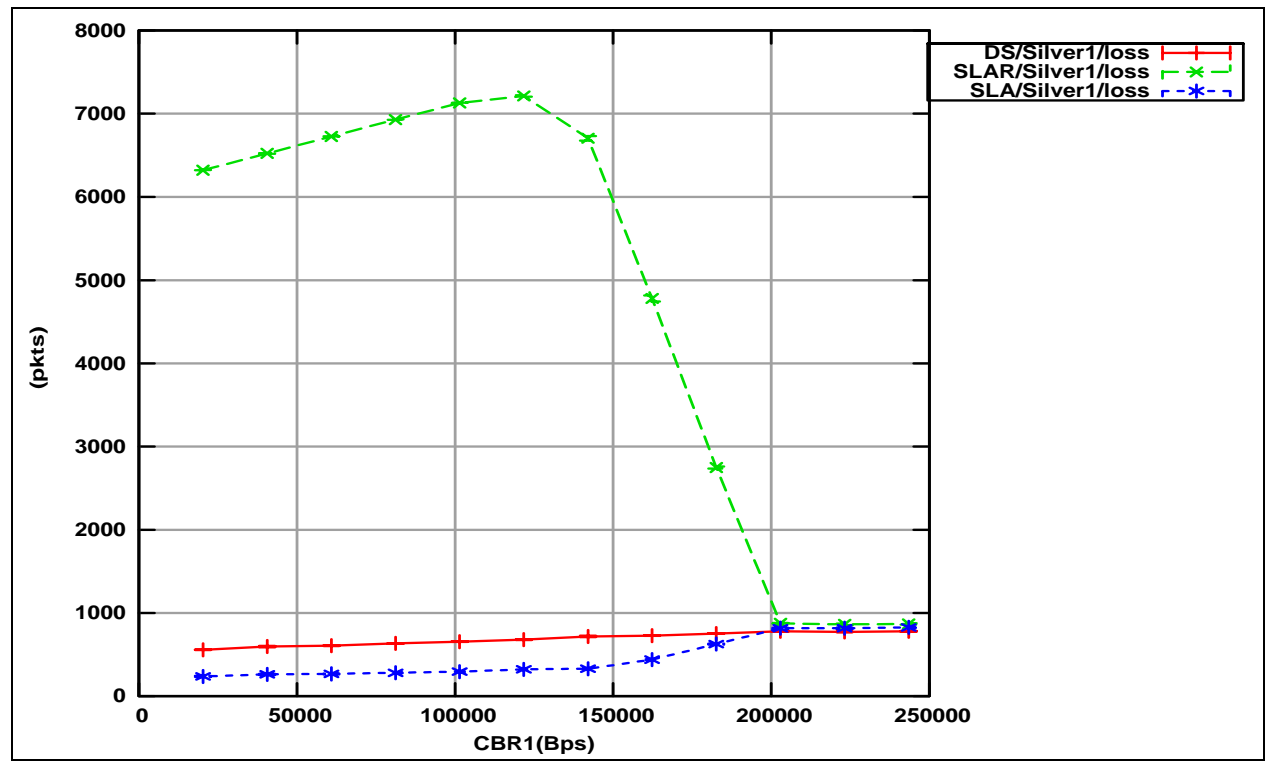

Figure 35 DS, SLAR, SLA Silver packet loss Versus Gold rate 


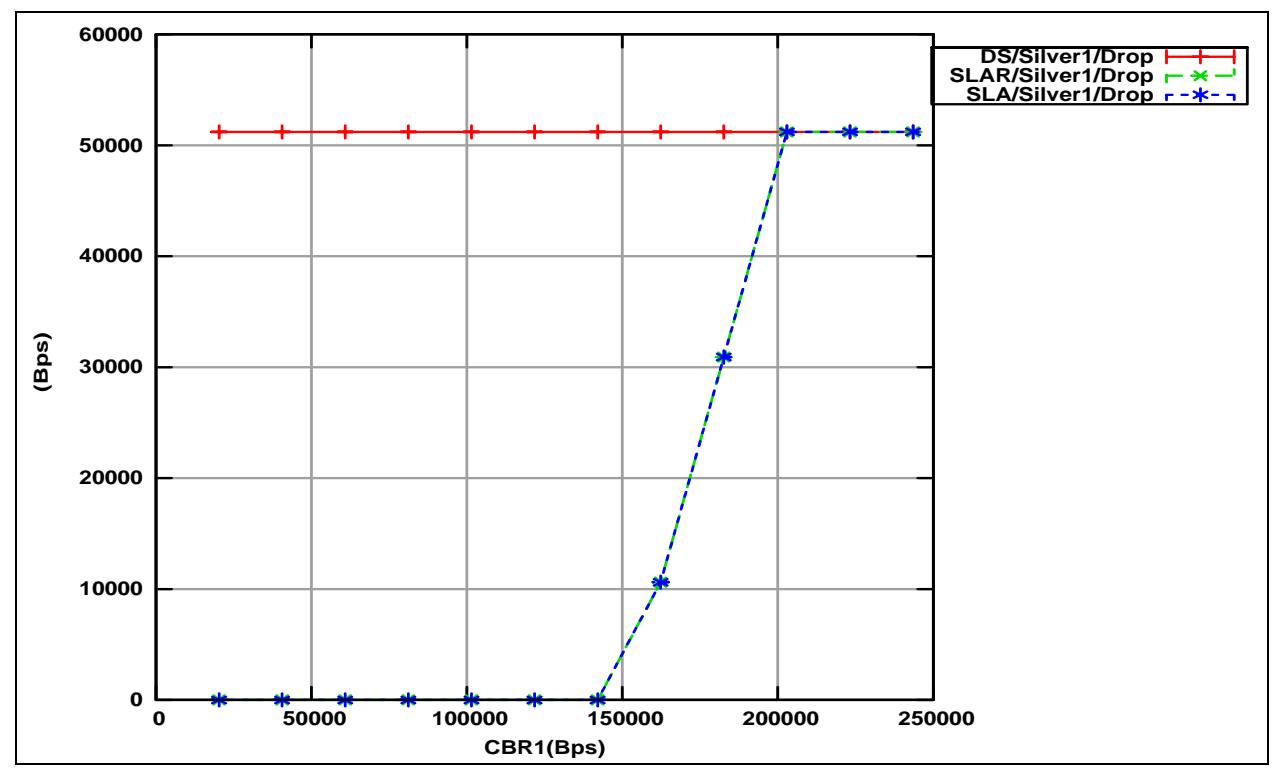

Figure 36 DS, SLAR, SLA Silver ingress drop Versus Gold rate

Figure 37 and Figure 38 show the losses and the drop rates, respectively, for Client1's Bronze rate. The figures show that the 3-tier SLA model has a clear advantage over the other 2 models as shown above for the Silver traffic.

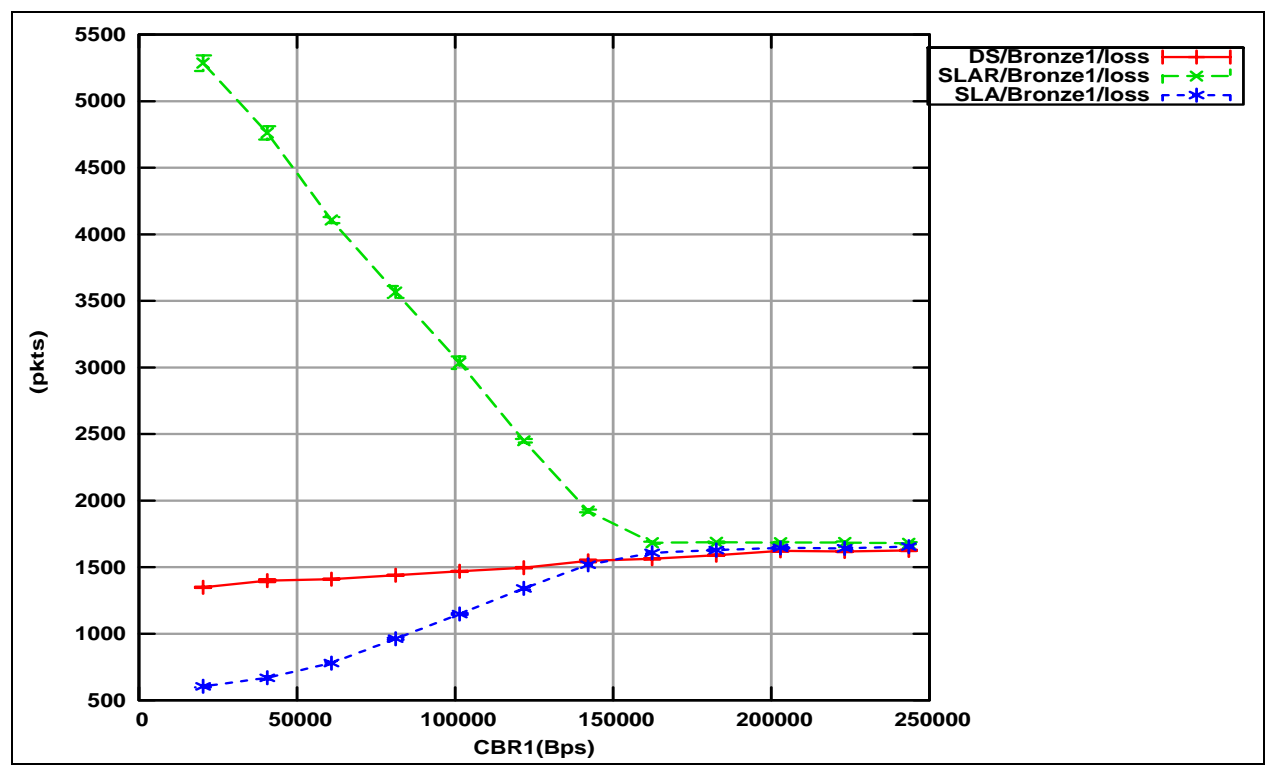

Figure 37 DS, SLAR, SLA Bronze packet loss Versus Gold rate 


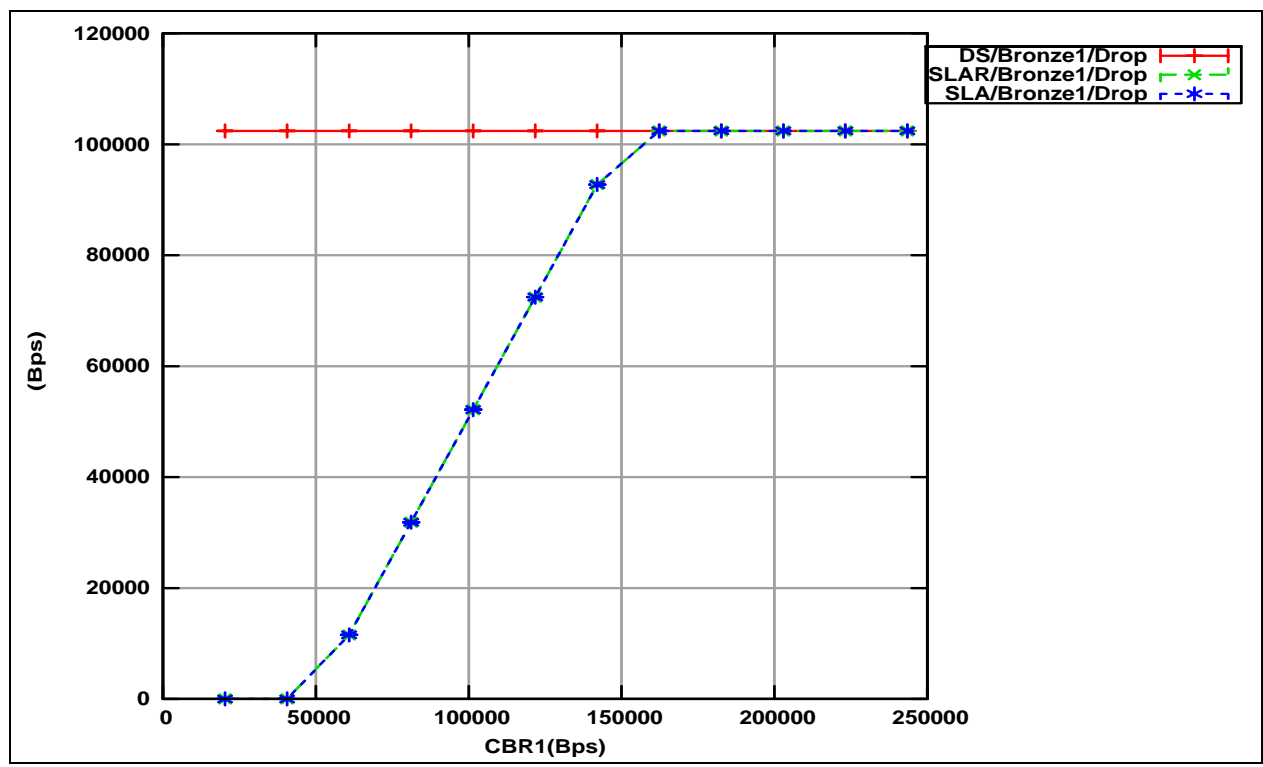

Figure 38 DS, SLAR, SLA Bronze ingress drop Versus Gold rate

Varying the Silver rate and plotting the Bronze packet losses (Figure 39) and the ingress rate drop due to policing (Figure 40), we can see that the SLAR model suffers the most due to reordering as shown in Figure 24. The DS ingress policing trims the Bronze excess traffic regardless of the availability of bandwidth in the upper classes. 


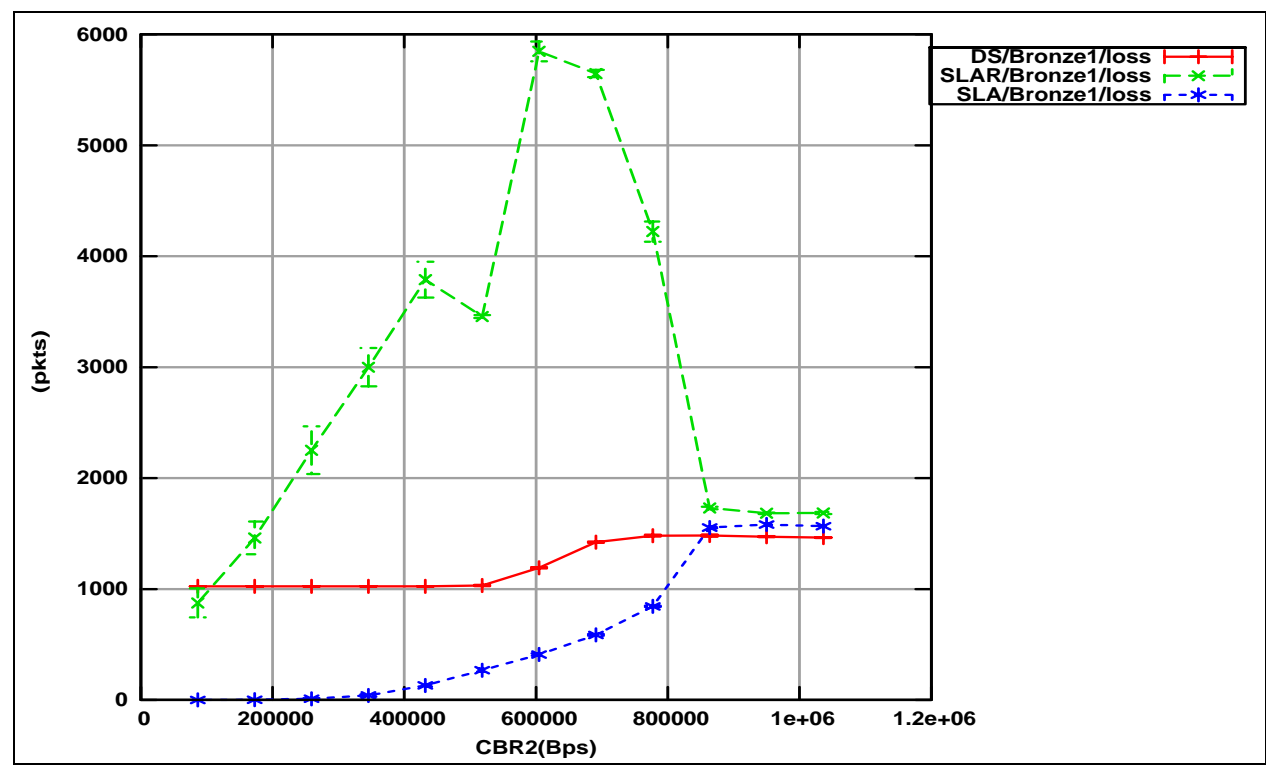

Figure 39 DS, SLAR, SLA Bronze packet loss Versus Silver rate

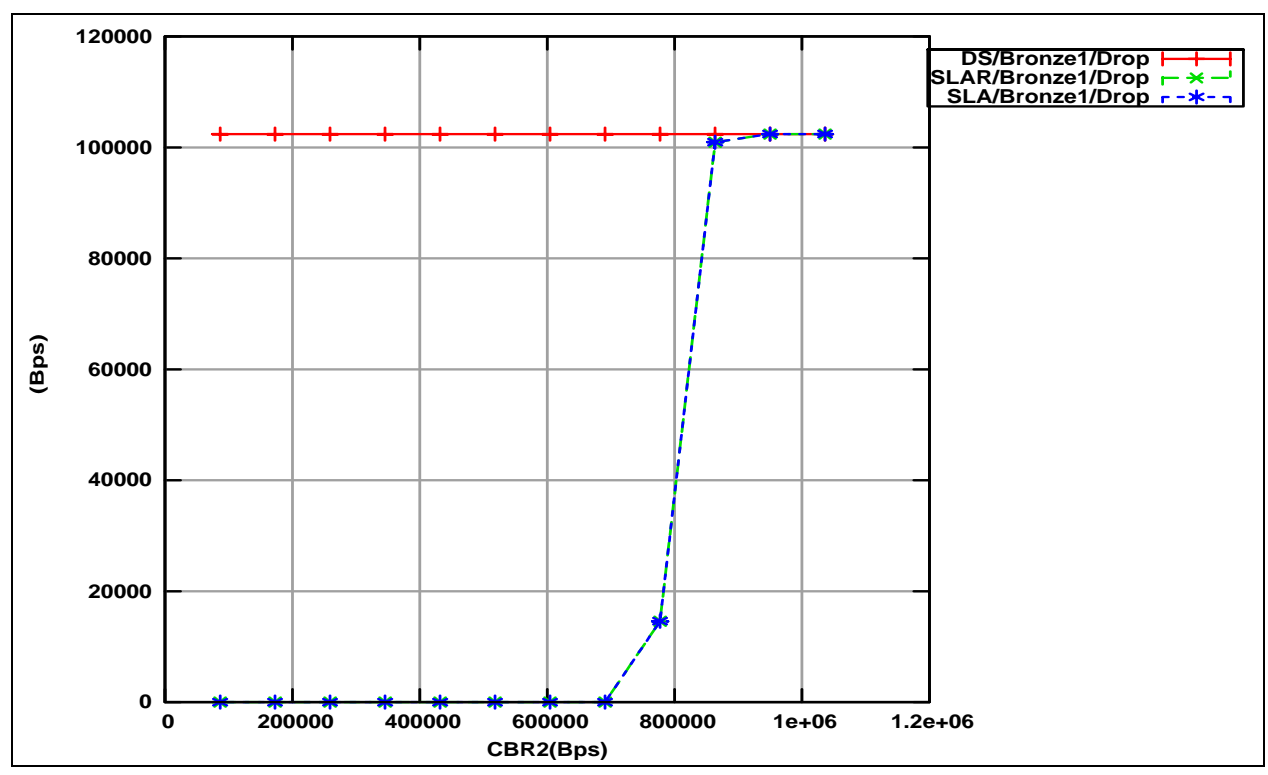

Figure 40 DS, SLAR, SLA Bronze ingress drop Versus Silver rate

\subsubsection{End-to-End Delay}

We have seen so far that the 3-tier SLA outperforms the DS and SLAR models in terms of goodput and packet loss. We will see in this section that the added end-to-end delay 
induced by accepting more packets (the upgraded packets) into the higher classes is still within the class' acceptable range.

In general, when a customer buys a certain bandwidth of a certain class of service, he expects the service provider, depending on the class of service, to guarantee a maximum End-to-End delay of all packets destined for that class, regardless of the bandwidth bought. For example, when a customer buys 10MBps of Gold service, he expects that all Gold packets should reach the destination within a certain pre-contracted time that the provider agreed to in the SLA, regardless of the amount of Gold traffic that the customer pushes as long as it fits within the 10MBps contracted rate.

In the following figures, we will compare the SLAR and the SLA models assuming that the maximum average delay observed in the DS model for a certain class defines the "acceptable" delay for that class. In other words, if any of the SLAR or the SLA models exceed the maximum observed DS delay for a certain class of service by a "significant" amount, then we will declare that the model may induce an additional delay for that class.

Figure 41 shows the End-to-End average Gold delay in ms along with the confidence intervals for the various models, when varying the Gold rate. The maximum observed DS delay is about $60.59 \mathrm{~ms}$. The SLAR model slightly exceeds this delay by about $0.03 \mathrm{~ms}$. Although $0.03 \mathrm{~ms}$ could be an acceptable or negligible added delay compared to the $60 \mathrm{~ms}$, as per our previous paragraph, we could declare that the SLAR model has induced an additional delay that exceeds the DS bounded delay. This delay is caused by upgraded packets on the ingress node, forwarded in the network from end-to-end as higher class packets, which will contribute to the observed delay in that class (in general, delay is composed of processing delay, queuing delay, propagation delay, and transmission delay; A higher ingress rate could 
cause additional queuing). Finally, the SLA model falls well within the DS maximum delay range considering confidence intervals even though there was some delay induced by upgraded packets. The main reason why the SLAR and the SLA differ, is that in the SLAR once packets are upgraded, they are upgraded from end to end. Whereas in the SLA model, packets are upgraded for a single hop, sharing tokens with other flows in the same aggregate which distributes the added delay over all paths in the network, particularly the background traffic flows (short background traffic added at each node), making the end-to-end delay shorter for that flow.

One might argue that since the DS model should guarantee the negotiated SLA per class delays when the network is fully loaded, then the 3-tier SLA maximum delays should fall well within these delay bounds. The 3-tier SLA does not allow, at any time, more traffic than the policed reserved bandwidths; however it does make sure that these bandwidths are fully used, as much as possible, by the customer.

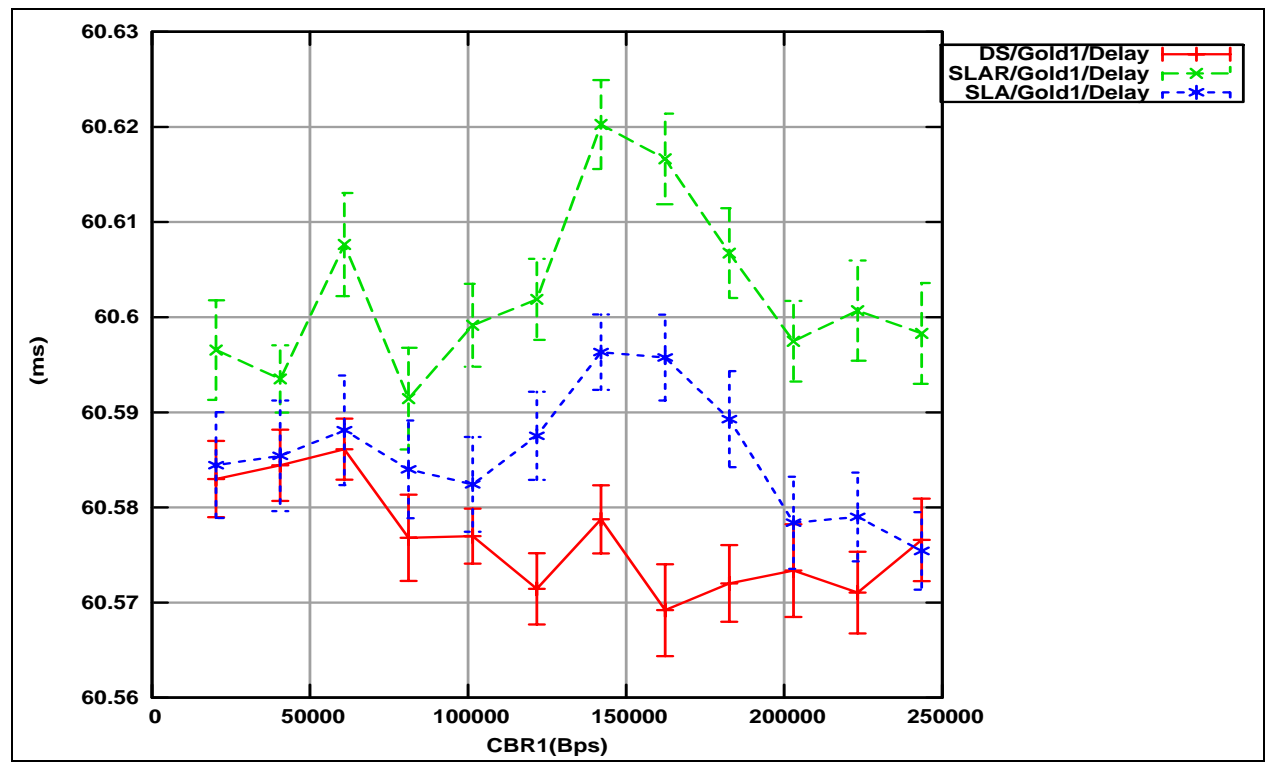

Figure 41 DS, SLAR, SLA End-to-End Gold delay Versus Gold rate 
Similarly, Figure 42 shows the End-to-End delay of the Gold traffic when varying the Silver rate. Again, the SLA model seems to have comparative delays with the DS model.

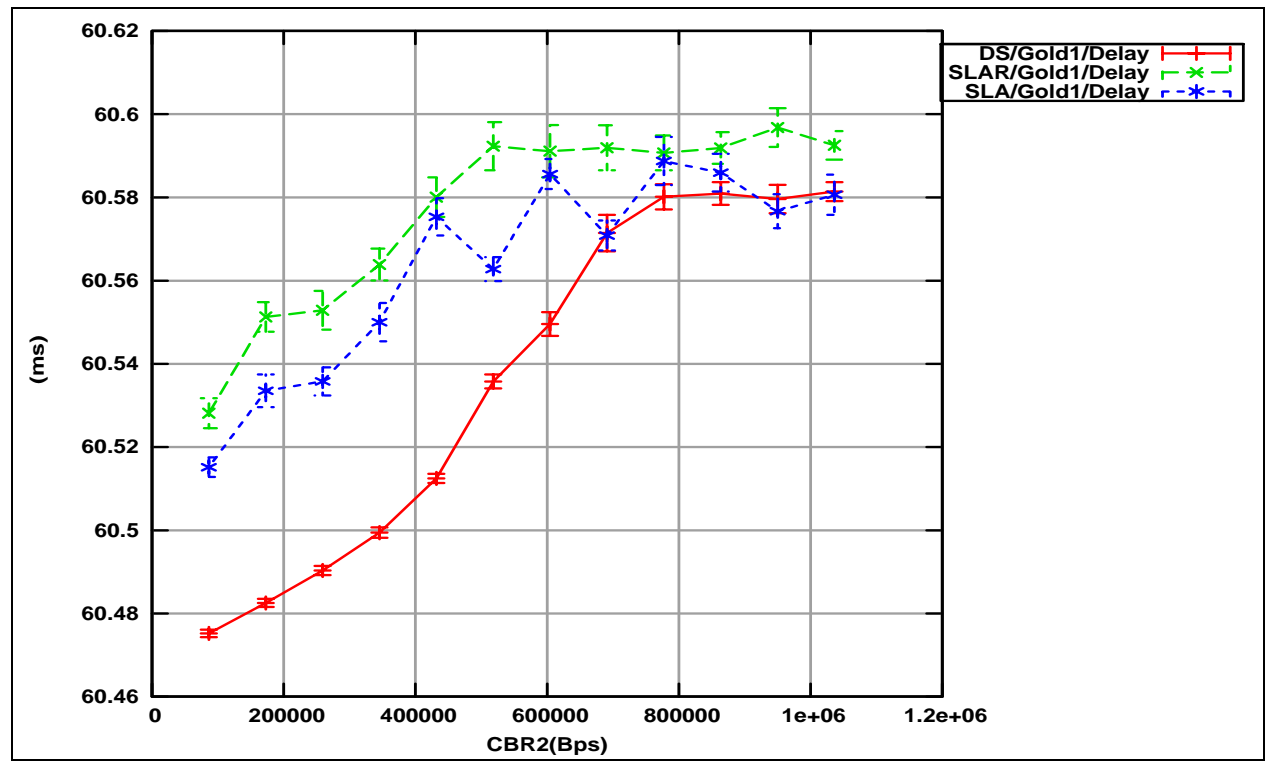

Figure 42 DS, SLAR, SLA End-to-End Gold delay Versus Silver rate

Figure 43 shows the Silver End-to-End delays of the three models. Obviously, the SLA delays fall well within the maximum DS delay. However, notice that the SLAR delays are significantly less than the other 2 models. This does not mean that the SLAR model has better delays; in fact, the measured SLAR delays are only for packets that did make it to the other end. Since the SLAR Silver traffic exhibited a lot of losses (due to reordering), as shown previously, the received packets, mostly upgraded to Gold packets, counted towards this "apparent" lower delay. 


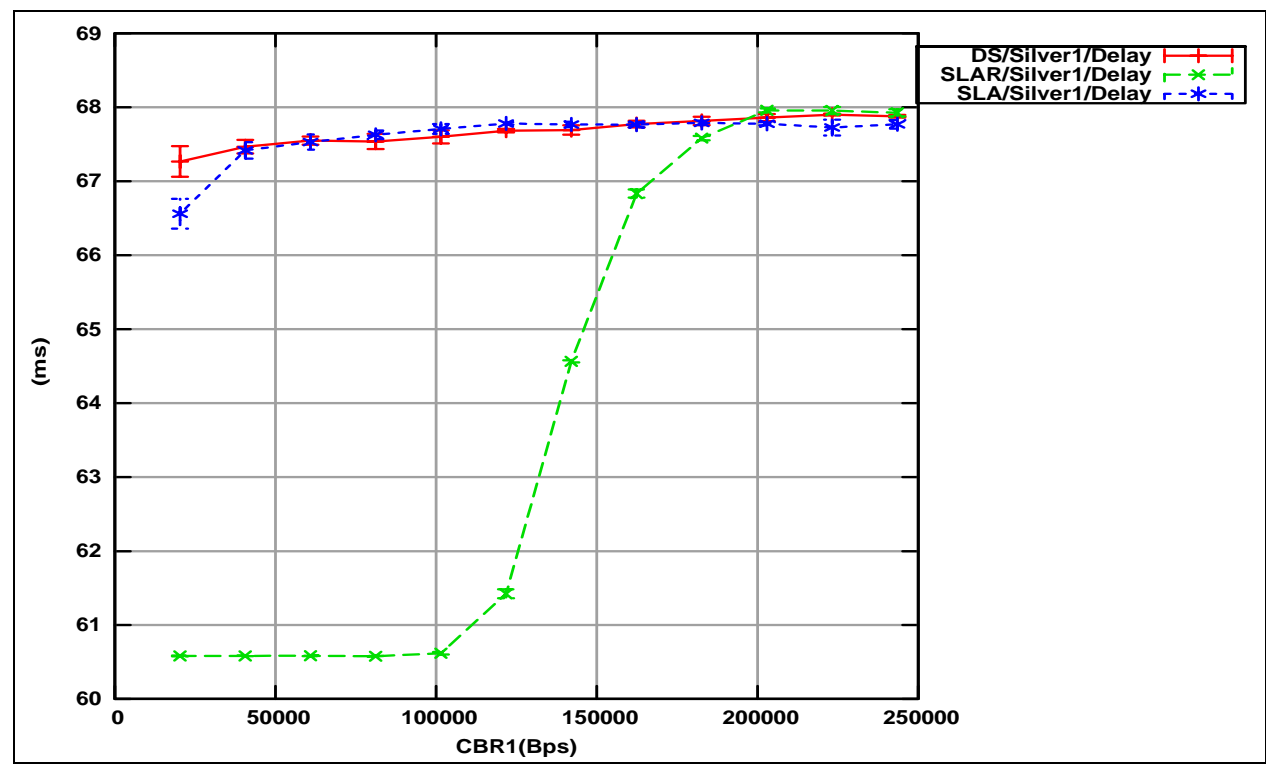

Figure 43 DS, SLAR, SLA End-to-End Silver delay Versus Gold rate

Similarly, Figure 44 shows Client1's End-to-End delay of the Silver traffic when varying the Silver rate.

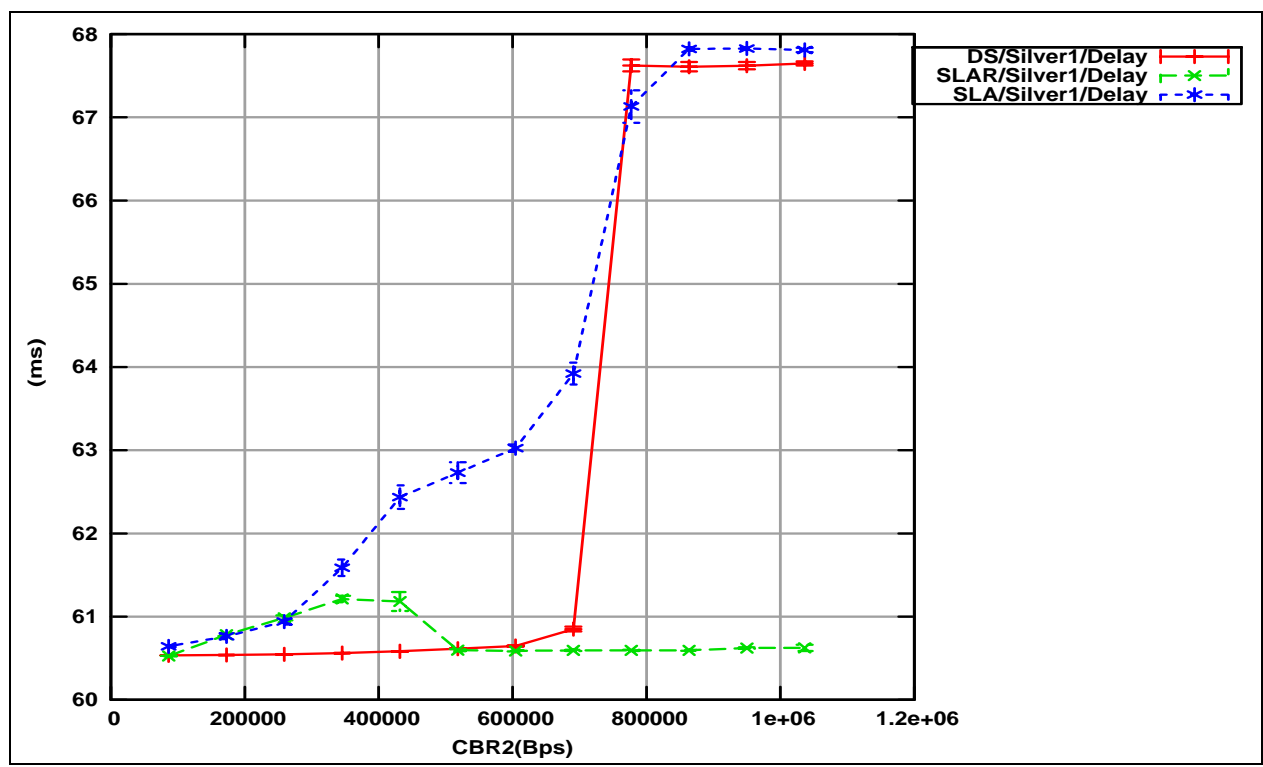

Figure 44 DS, SLAR, SLA End-to-End Silver delay Versus Silver rate

As for the Bronze delay, Figure 45, the losses in the SLAR model obscure the real delay at low Gold rates (notice that at high Gold rates, the SLAR model showed relatively 
low losses due to lower reordering). The higher delays at high Gold rates are due, for the same reasons mentioned above, to upgrading packets from End-to-End and from Client2 TCP retransmissions. As for the SLA model, it shows a bit higher delays (at low cbr1 rates) than the DS model, since it is accepting much more traffic than the DS model (DS model ingress policers prevent any extra Bronze traffic from passing through; in the SLA model, they are actually upgraded to Silver). However, in general, we can claim that the max delay in the SLA model is comparable to the max delay in the DS model.

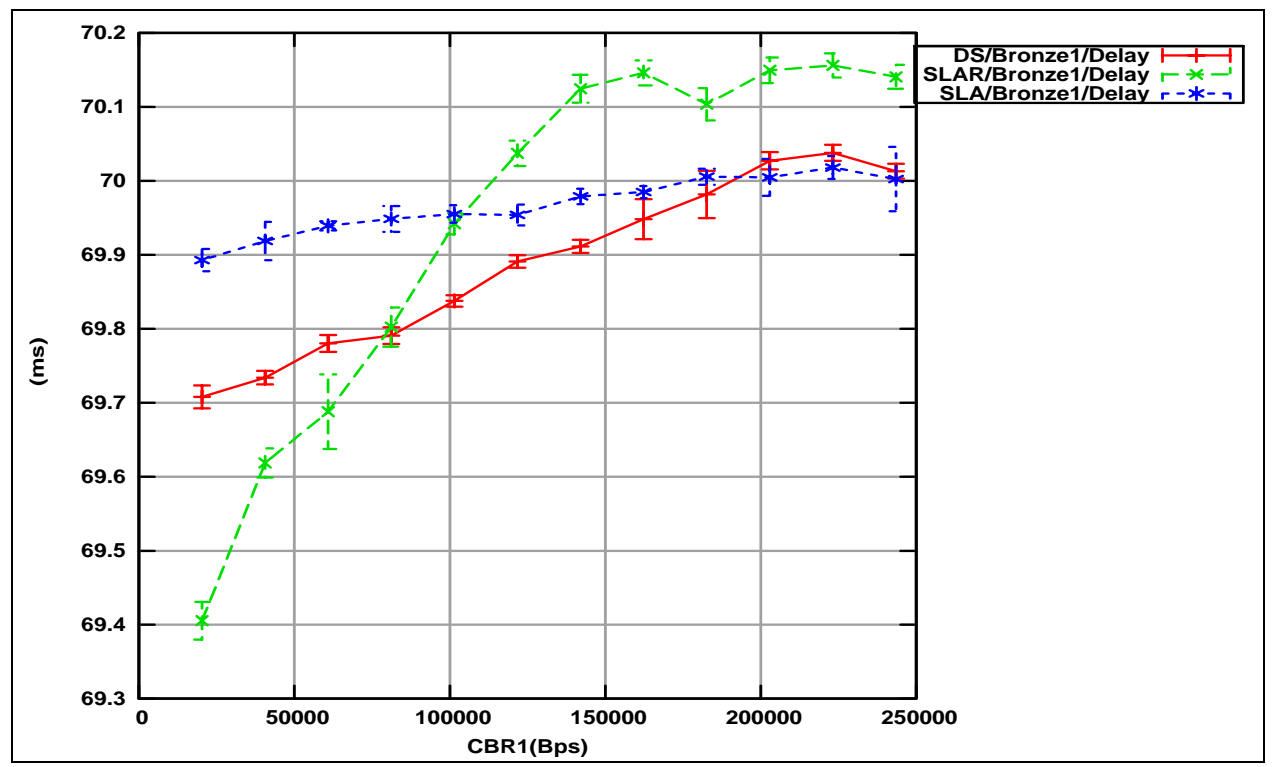

Figure 45 DS, SLAR, SLA End-to-End Bronze delay Versus Gold rate

Similarly, Figure 46 shows Client1's End-to-End delay of the Bronze traffic when varying the Silver rate. 


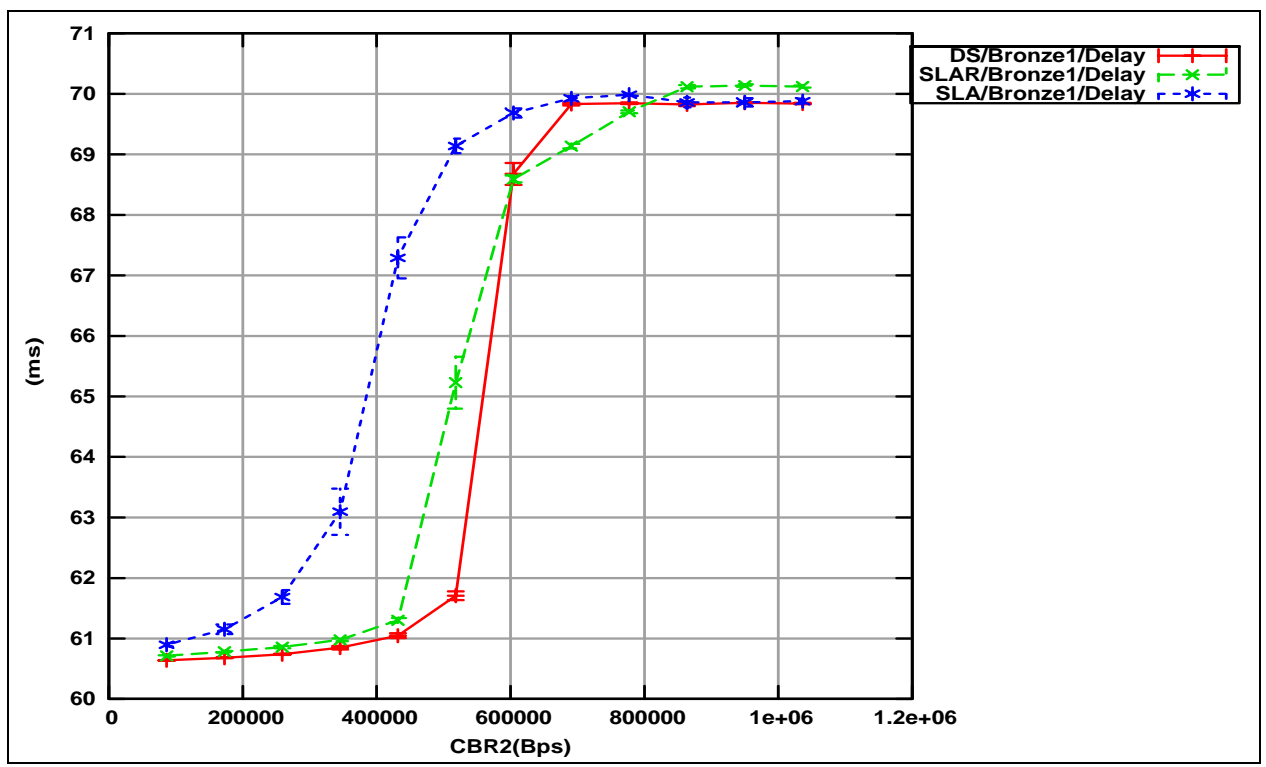

Figure 46 DS, SLAR, SLA End-to-End Bronze delay Versus Silver rate

\subsubsection{Network load}

We have seen so far how the automatic upgrades with the reordering solution benefit customers by trying, depending on the customers' offered load, to fully use their paid-for rates. In this section, we will investigate the effect of the network load on the 3-tier SLA when compared to the other 2 models i.e. DS and SLAR, by varying the End-to-End Gold background traffic or Client3's CBR (CBR7 in the NS-2 tcl code). Since Gold traffic is the highest quality class, reducing the Gold traffic in the network should benefit both the Silver and Bronze classes.

\subsubsection{Effect of Network load on reordering}

Since Client1's ingress rates are not changed, the throughput at the ingress node for all 3 models for all 3 service classes is the same. However, the Silver and Bronze goodput at the receive side are affected by the network load and are shown in Figure 47 and Figure 48 for all 3 models. The Gold goodput is the same for all 3 models and is not shown. 


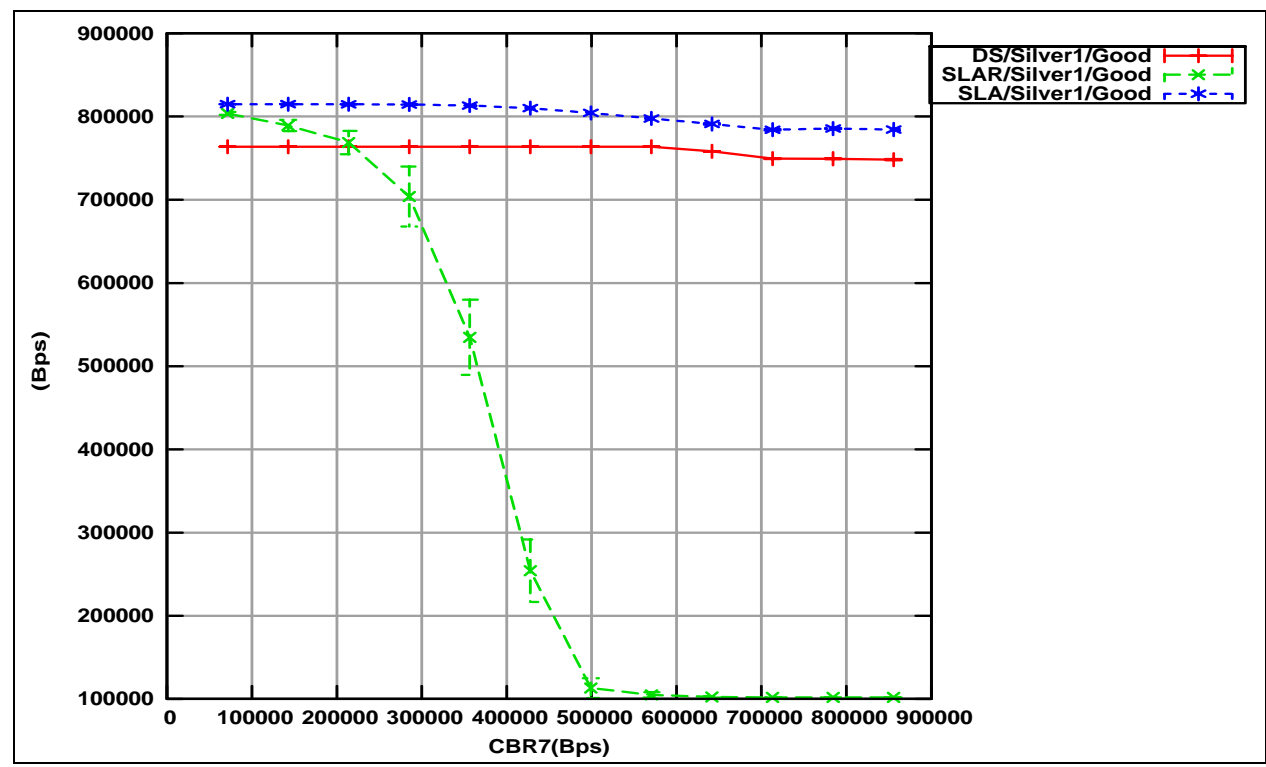

Figure 47 DS, SLAR, SLA Silver goodput Versus Background Gold rate

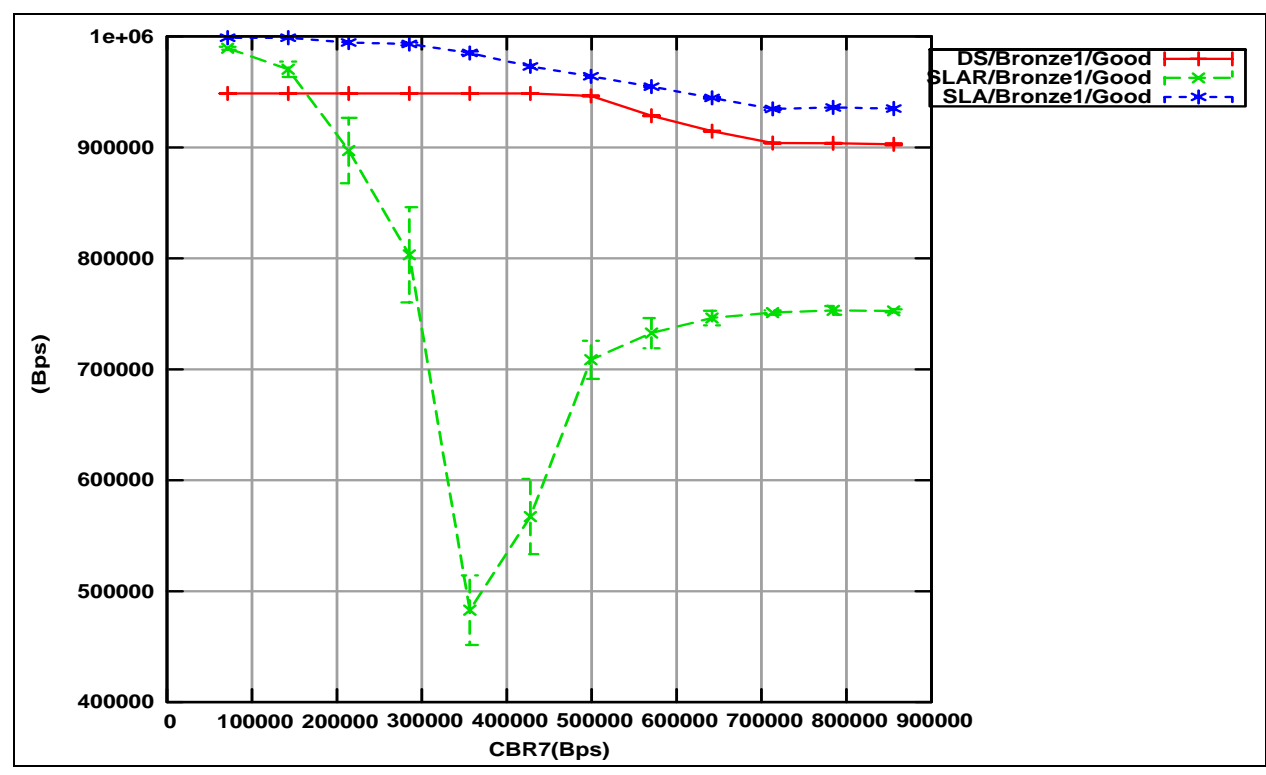

Figure 48 DS, SLAR, SLA Bronze goodput Versus Background Gold rate

The SLA goodput for both Silver and Bronze is better than the other 2 models due to upgrades on ingress and no reordering in the network. The SLAR behavior is explained by the amount of reordering and losses in the network as shown in Figure 49 and Figure 50. For the Silver case, as the background Gold rate increases, the Silver queues in the network build 
up (higher classes get better service at the expense of lower classes). The bigger the queues, the higher is the delay for a Silver packet to get serviced and the higher the probability of dropping the packets due to RED buffer management. The higher the delay, the higher is the reordering since all the upgraded packets will bypass the previous packets waiting in the Silver queue. Notice in Figure 47 that the upgraded Silver packets (about $\sim 100 \mathrm{KBps}$ ) manage to bypass the Silver congestion since they are flowing as Gold packets in the network.

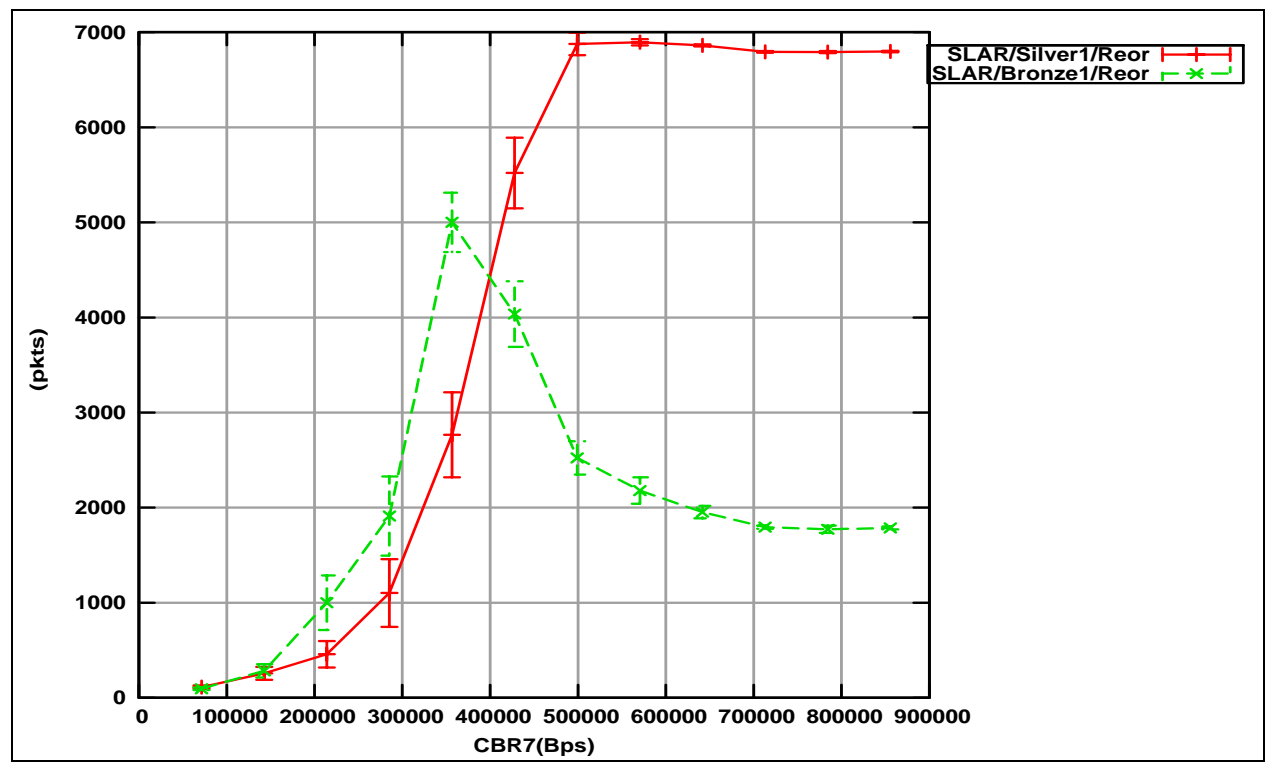

Figure 49 SLAR Silver and Bronze reordering Versus Background Gold rate 


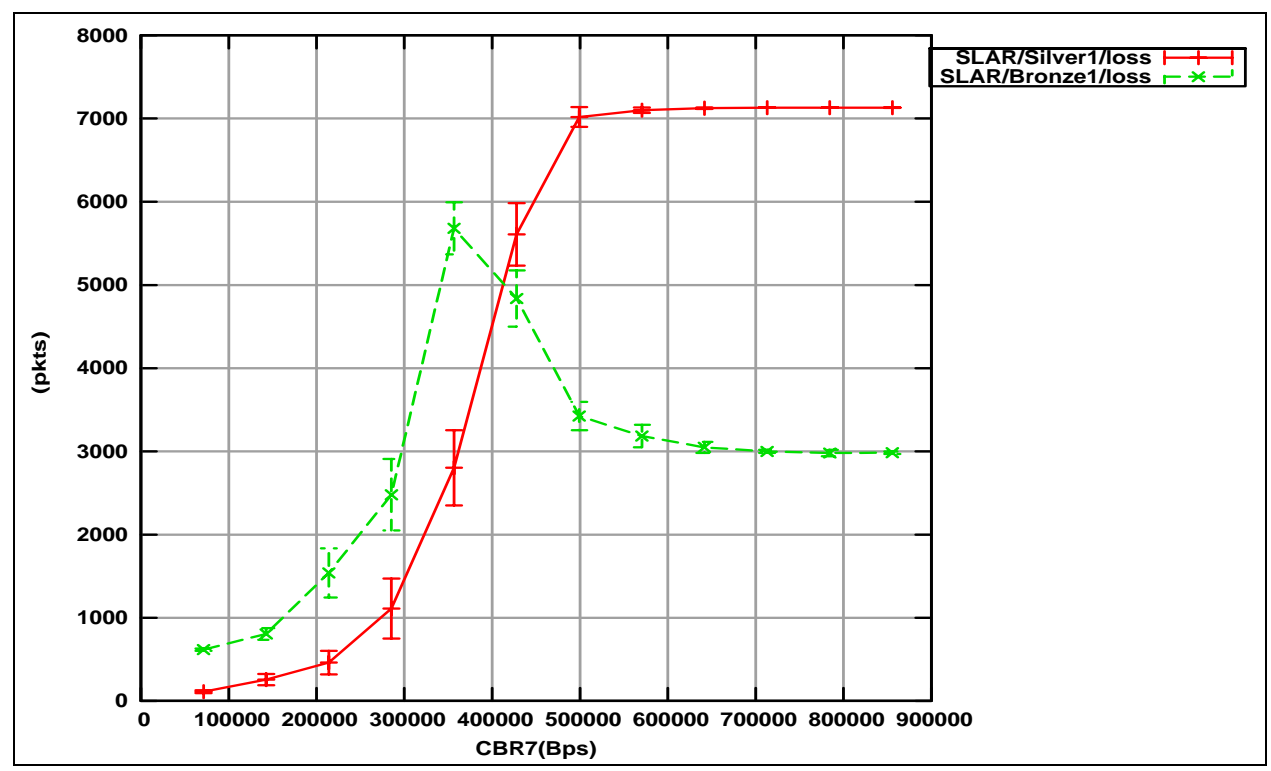

Figure 50 SLAR Silver and Bronze losses Versus Background Gold rate

Figure 51 and Figure 52 show the real time queue sizes and the RED average queue sizes for the cases when CBR7 is 71303Bps and 855636Bps, respectively. Figure 53 and Figure 54 show the corresponding loss rates due to RED buffer management algorithm. Notice from Table 7 that RED is configured to drop with a probability of $100 \%$ when the Silver average queue size exceeds 20 packets (that is why the RED average does not exceed 20 in Figure 52). 


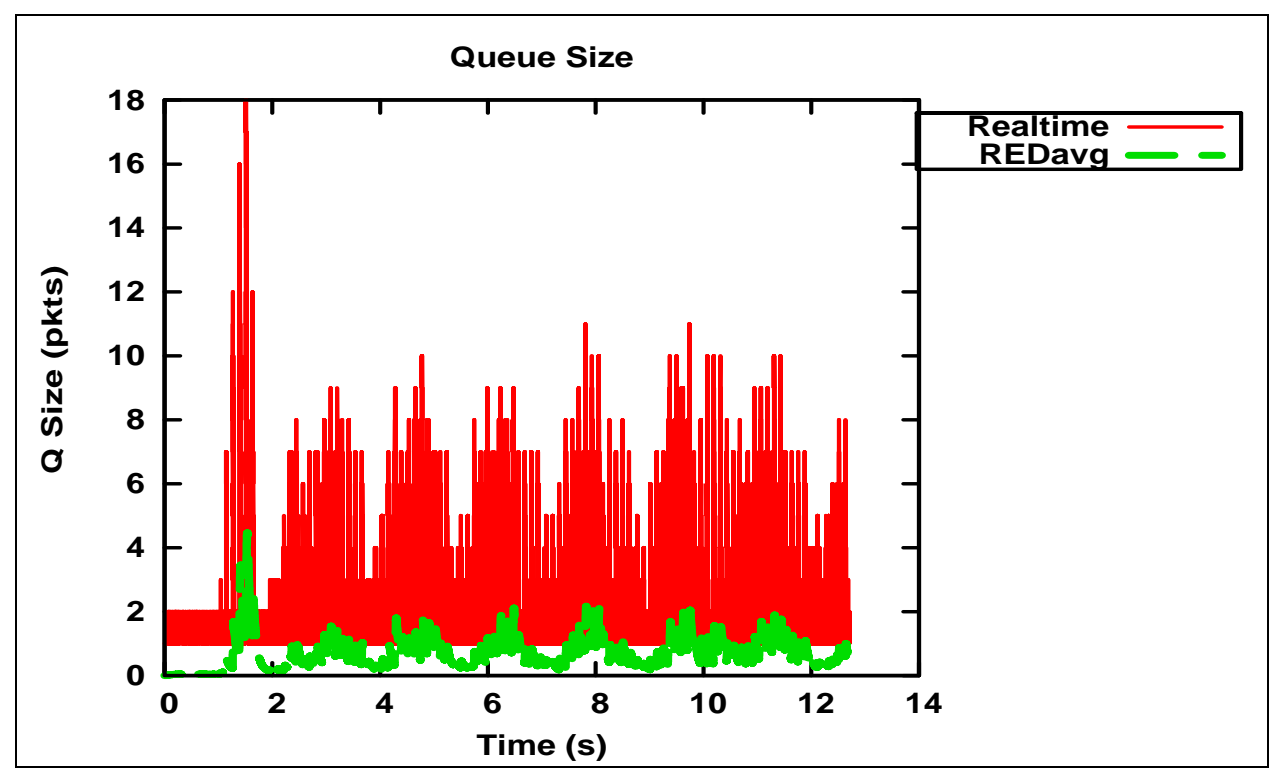

Figure 51 SLAR n_n2 Silver Queue Size for CBR7=71303Bps

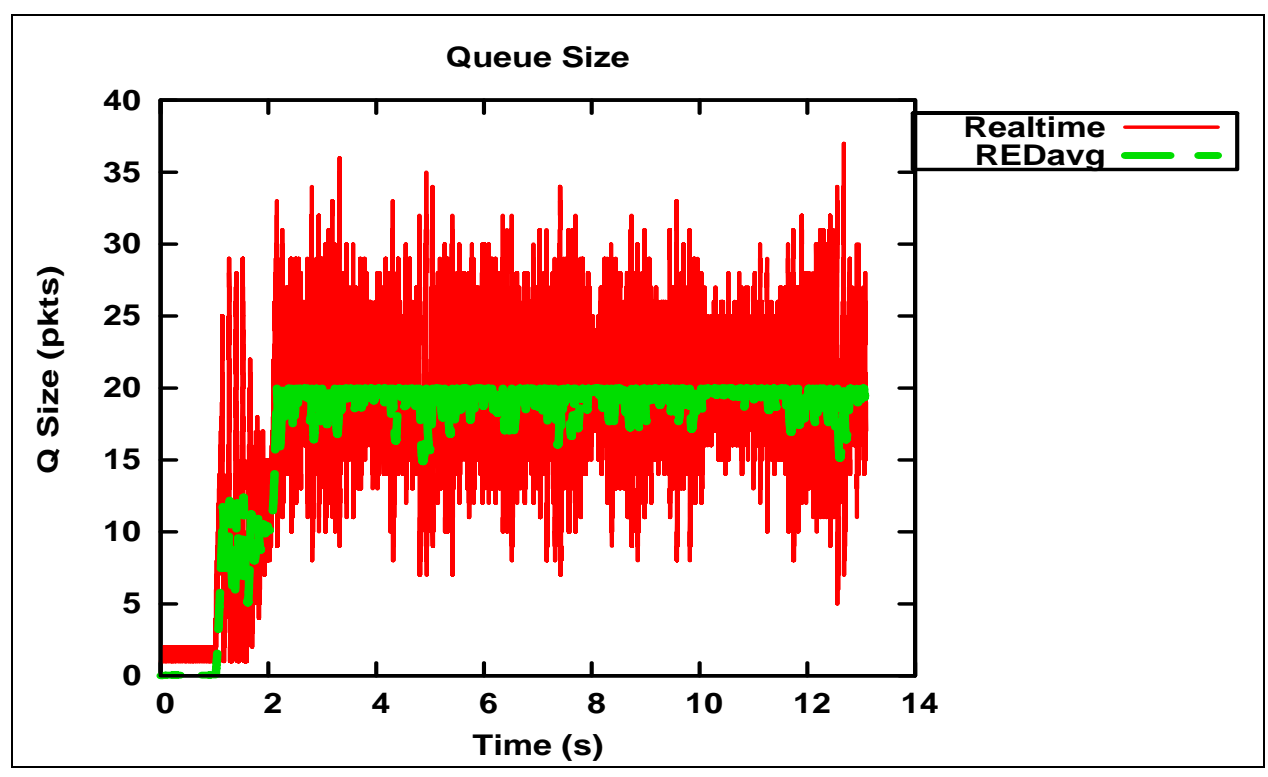

Figure 52 SLAR n_n2 Silver Queue Size for CBR7=855636Bps 


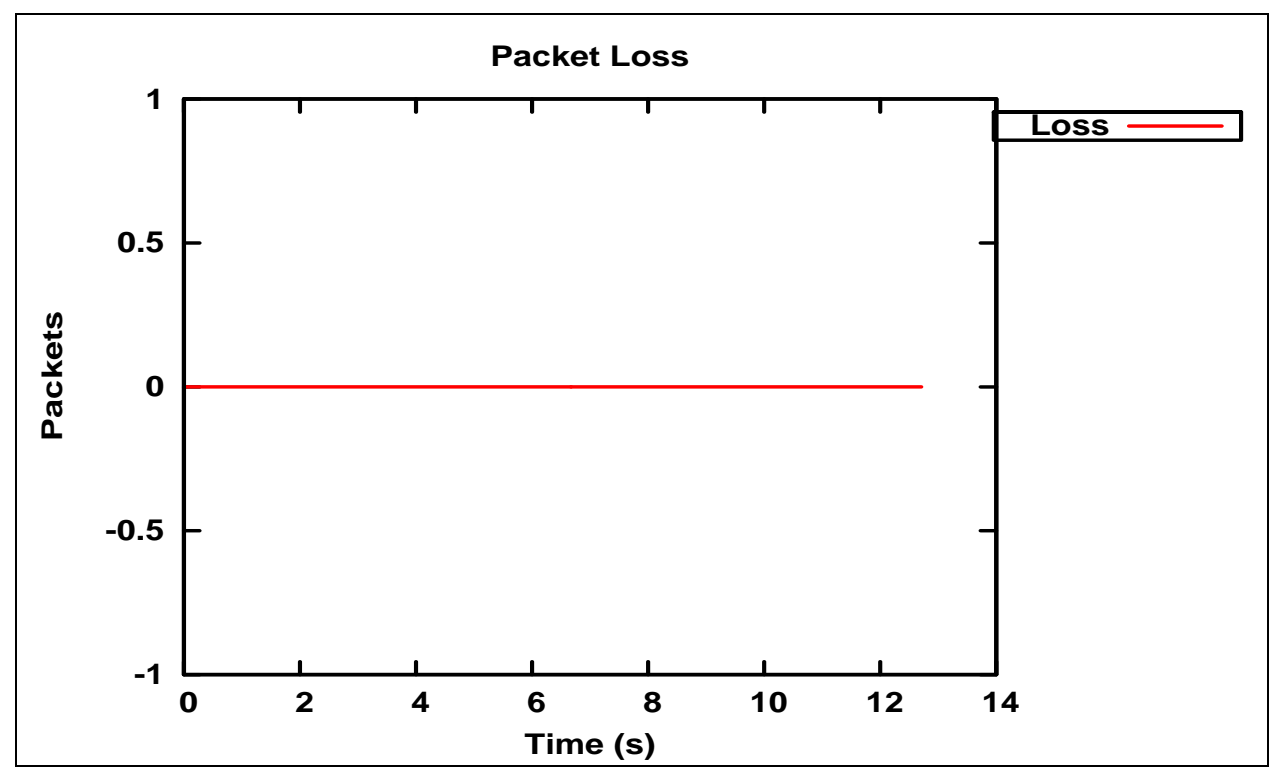

Figure 53 SLAR n_n2 Silver Queue RED losses for CBR7=71303Bps

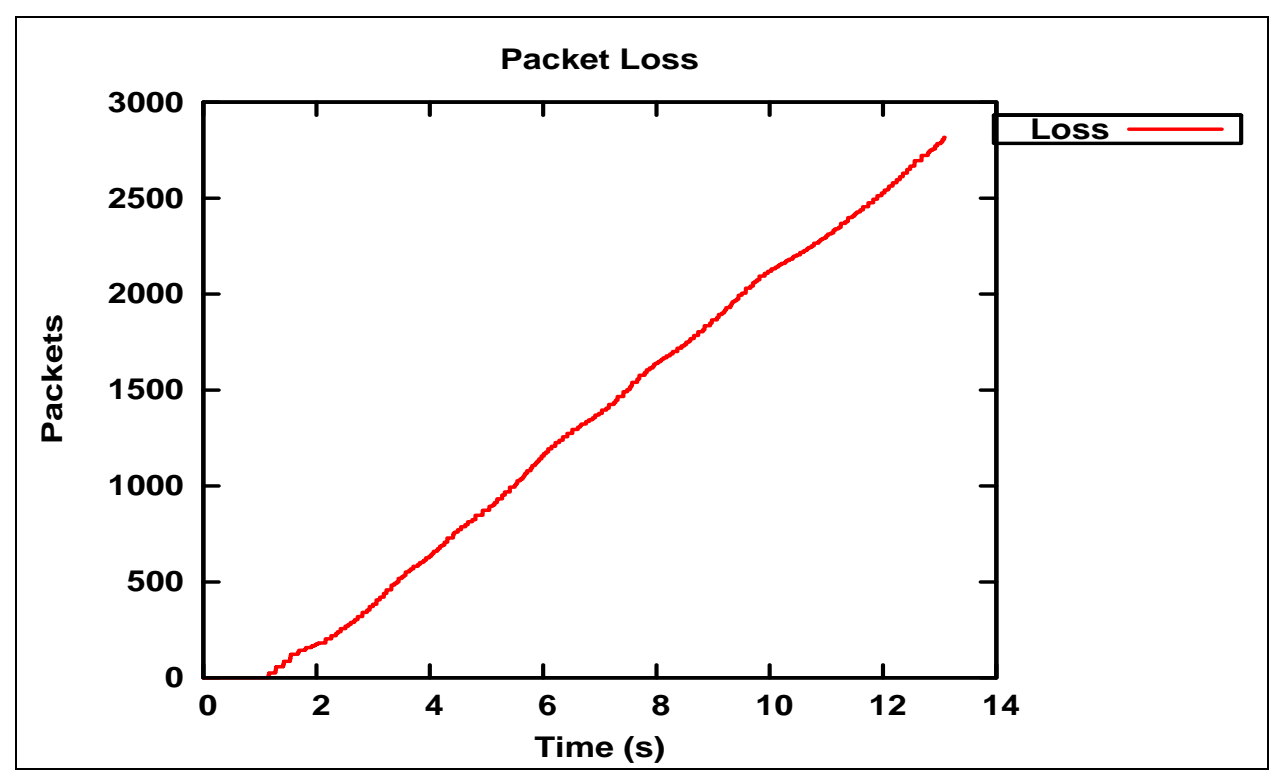

Figure 54 SLAR n_n2 Silver Queue RED losses for CBR7=855636Bps

Figure 49 and Figure 50 show that the Bronze traffic peaks with heavy reordering (or heavy losses) at a certain point. The reason has to do with the Bronze relative queue size with respect to the Silver. When both queue sizes are small, or when both queue sizes are large, we should expect relatively lower reordering than when the Silver is small and the Bronze is 
large. This is due to the fact that for reordering to occur, upgraded packets need to bypass non-upgraded packets in the network. The probability for an upgraded packet to bypass nonupgraded packets is the highest when the upgraded packet observes low queuing in the network and that the non-upgraded packets observe high queuing. So, the highest probability of reordering occurs when the Silver queue size is small compared to the Bonze queue size. Indeed checking the queue sizes as the background Gold traffic increases, both Silver and Bronze start with relatively low queue sizes (Figure 55), with the Bronze getting congested first (Figure 56). Then once the Bronze queue is congested, the Silver queue starts filling up to end up with both queues relatively full (Figure 57). This difference in queue sizes explains the Bronze loss behavior peaking out when the Silver queue size is low and the Bronze queue size is high.
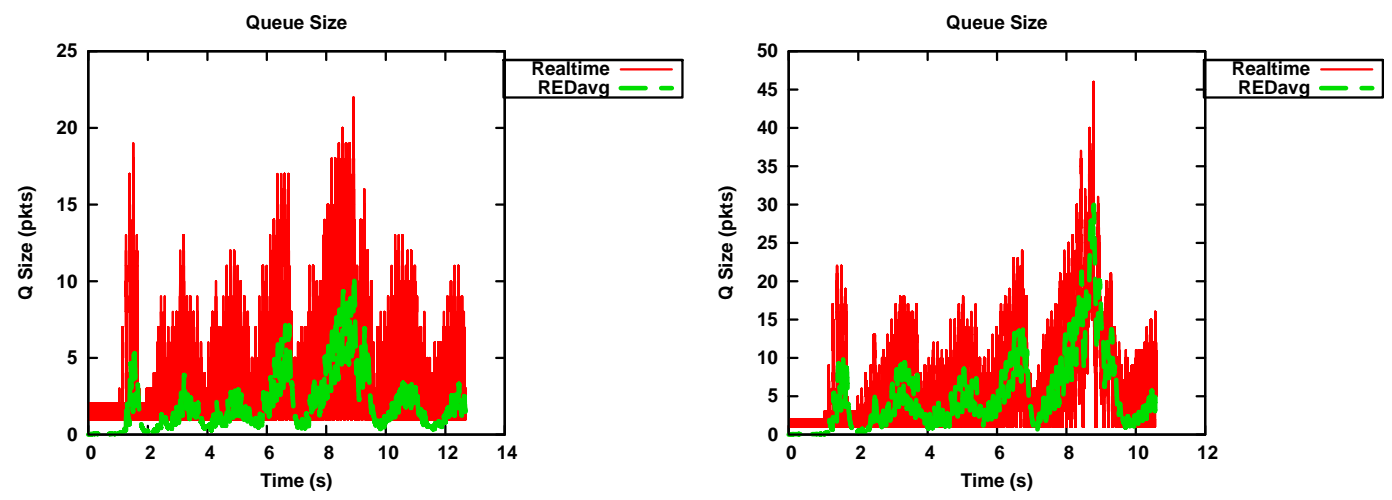

Figure 55 Silver (left) and Bronze (right) n_n2 queue sizes at cbr7=213909 Bps 

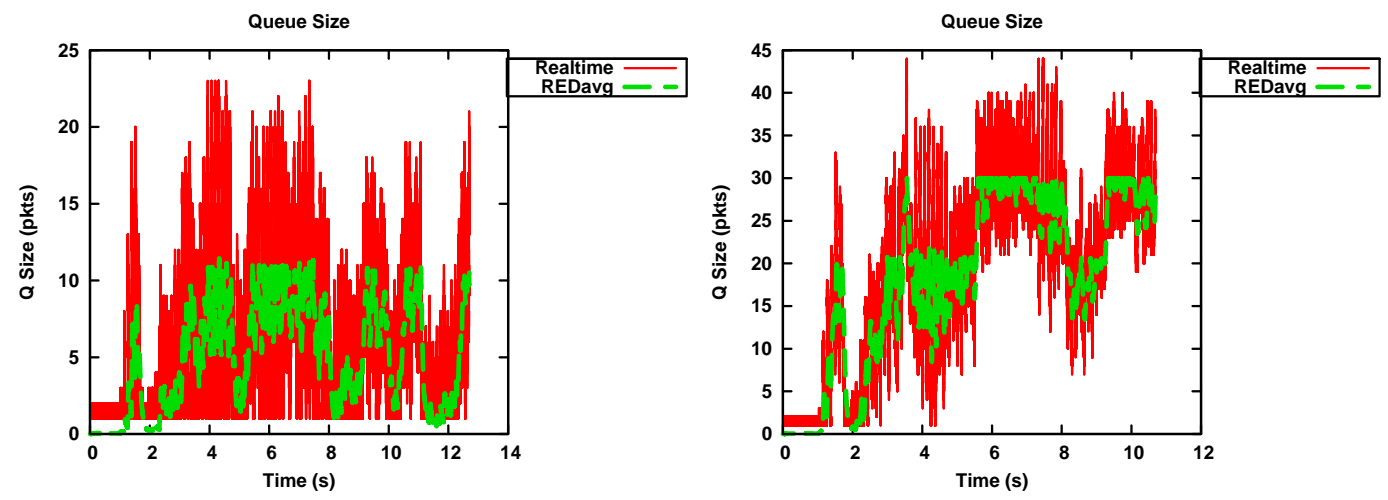

Figure 56 Silver (left) and Bronze (right) n_n2 queue sizes at cbr7=356515 Bps
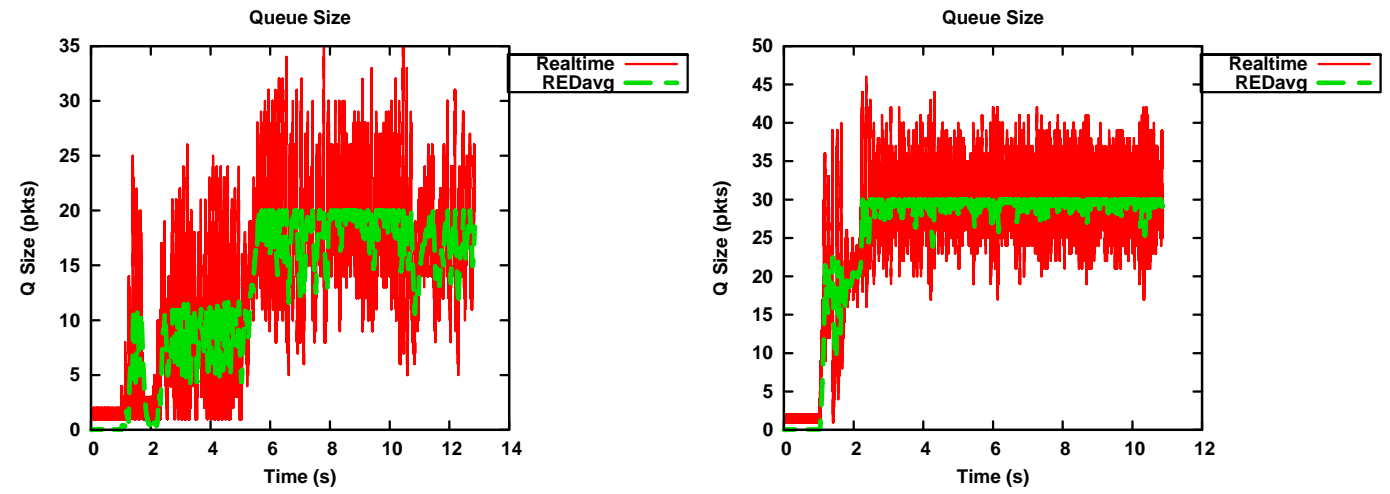

Figure 57 Silver (left) and Bronze (right) n_n2 queue sizes at cbr7=499121 Bps

Figure 58, Figure 59, and Figure 60 show the average Gold, Silver and Bronze delays, respectively, of the 3 models when varying the background Gold traffic rate. The figures show that the DS model has the lowest delays since it accepts the least amount of traffic due to tight ingress policing whereas the other 2 models allow for upgrades and thus more traffic (more traffic means bigger queues, which means larger queuing delays). The Silver SLAR plot shows an "apparent" lower delay due to higher drops in the network. In fact, only a few SLAR Silver packets made it, the ones that were upgraded to Gold on ingress (these upgraded packets contributed to a lower delay since they were treated as Gold from End-toEnd). The other Silver packets were reordered and not accounted for (considered losses) 
when computing the average delays. Finally, the SLA model shows higher delays than the DS model but the maximum delays fits well within the DS maximum delay boundaries.

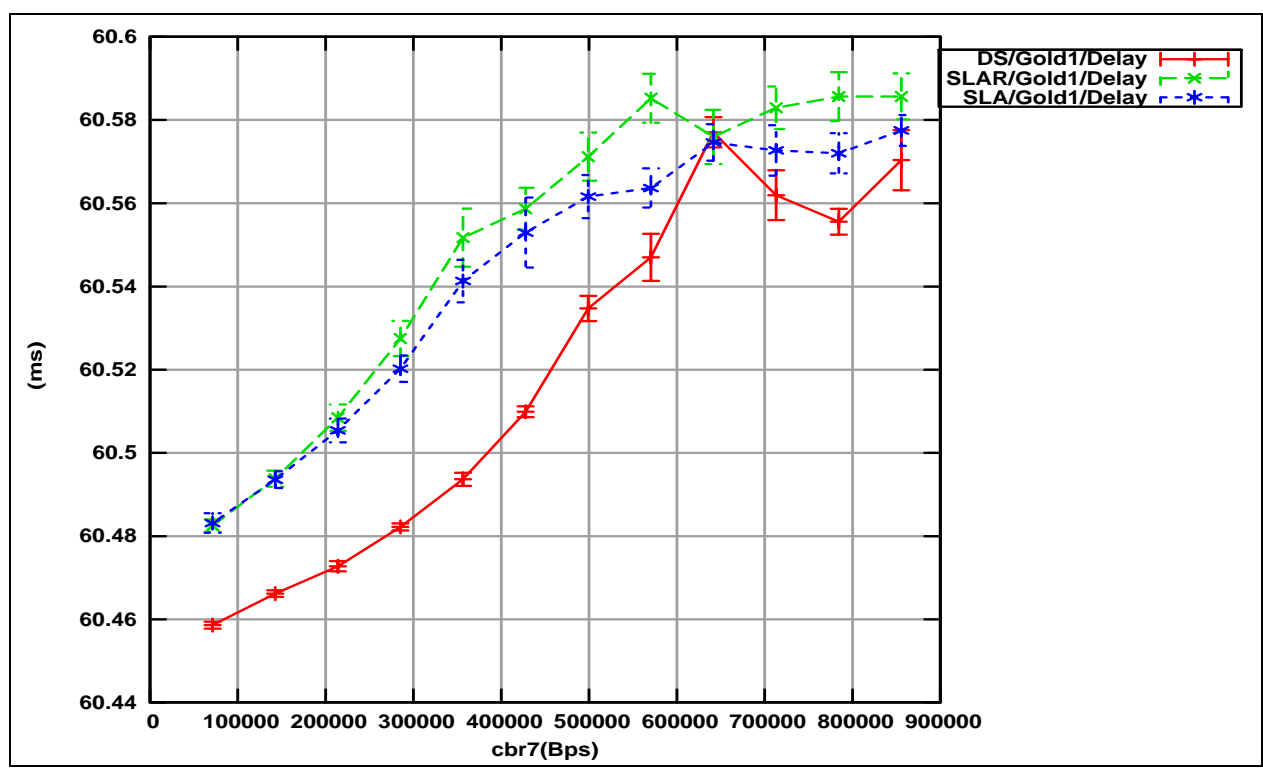

Figure 58 DS, SLAR, SLA Gold average delay Versus Background Gold rate

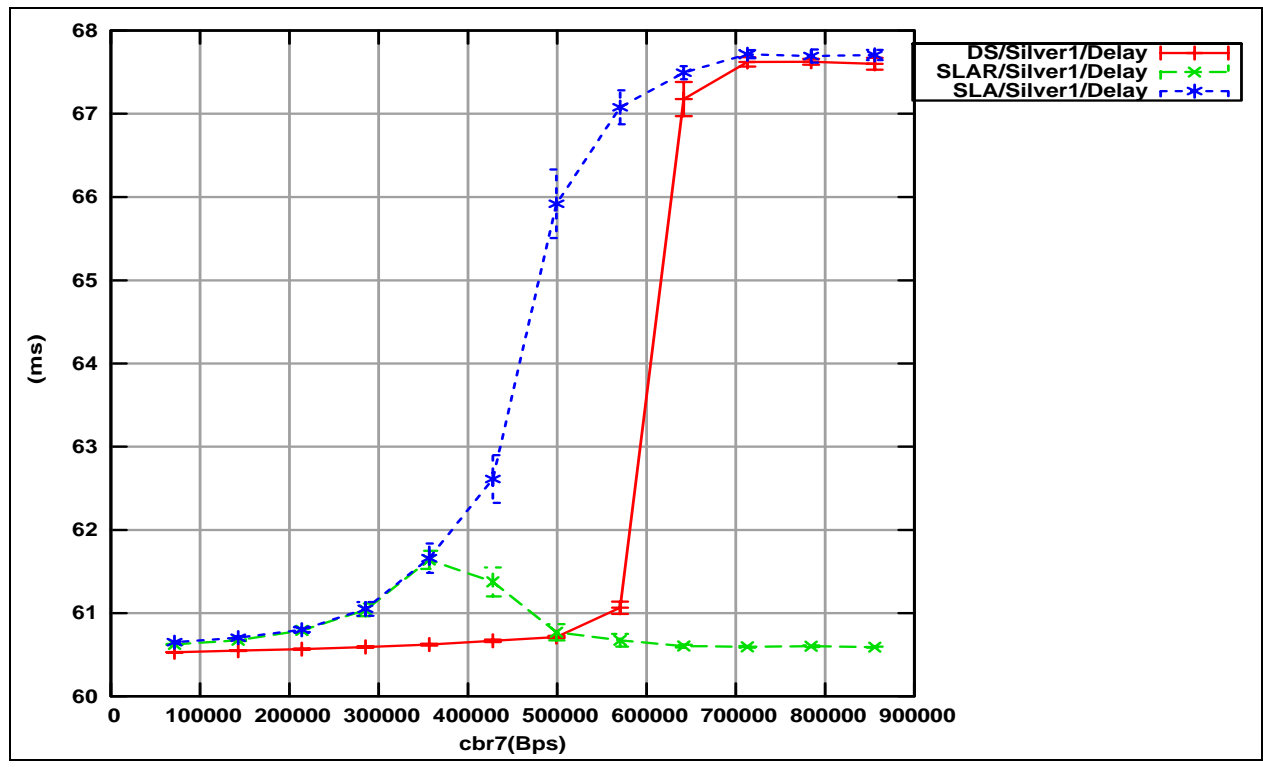

Figure 59 DS, SLAR, SLA Silver average delay Versus Background Gold rate 


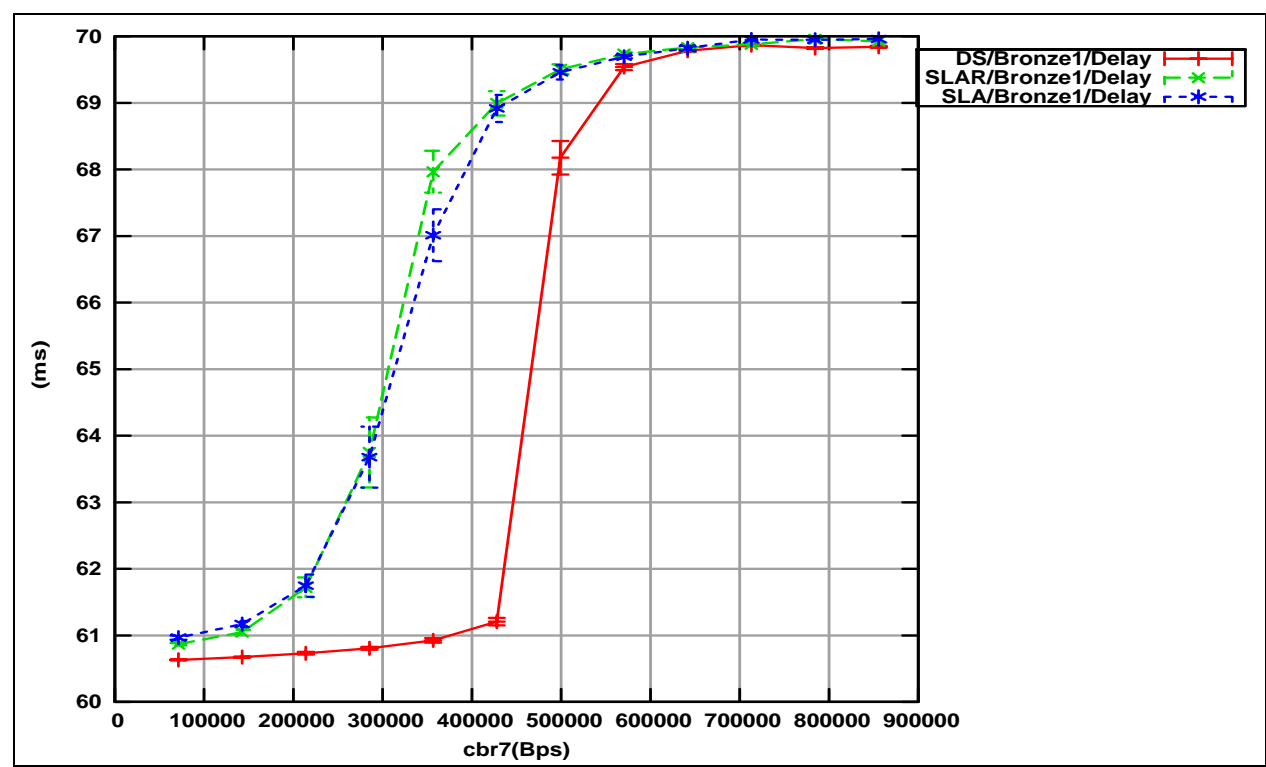

Figure 60 DS, SLAR, SLA Bronze average delay Versus Background Gold rate

\subsubsection{Low Versus High Network load}

We have seen, so far, the behavior of the 3 models under heavy network load (section 6.4.1) and we have concluded that the SLA model provides the most benefit for the customer. We will see in this section, where we re-run the simulation varying Client1's Gold traffic with low network load, that although the SLAR model shows some improvement the SLA model is still the most promising.

In this experiment, we will reduce the Gold End-to-End background traffic (called CBR7 in the NS-2 TCL code) to 71,303Bps which will relax the network load as shown in Figure 51, where the Silver queue is plotted over time (the figure shows that there was not enough traffic in the network to cause congestion).

Figure 61 shows Client1's Silver goodput of the DS, SLAR and SLA models. Compared to Figure 27 on page 93, the Silver goodput is even better than the DS model. This is due to the upgrades on ingress and the relatively lower level of reordering as shown in 
Figure 62, which shows the number of packets reordered under both high network load $(\mathrm{CBR} 7 \mathrm{rate}=713,031 \mathrm{Bps})$ and low network load $(\mathrm{CBR} 7 \mathrm{rate}=71,303 \mathrm{Bps})$. As observed in the figure, the low network load did not completely eliminate reordering, but it reduced it so that it barely affected the goodput on the receive side.

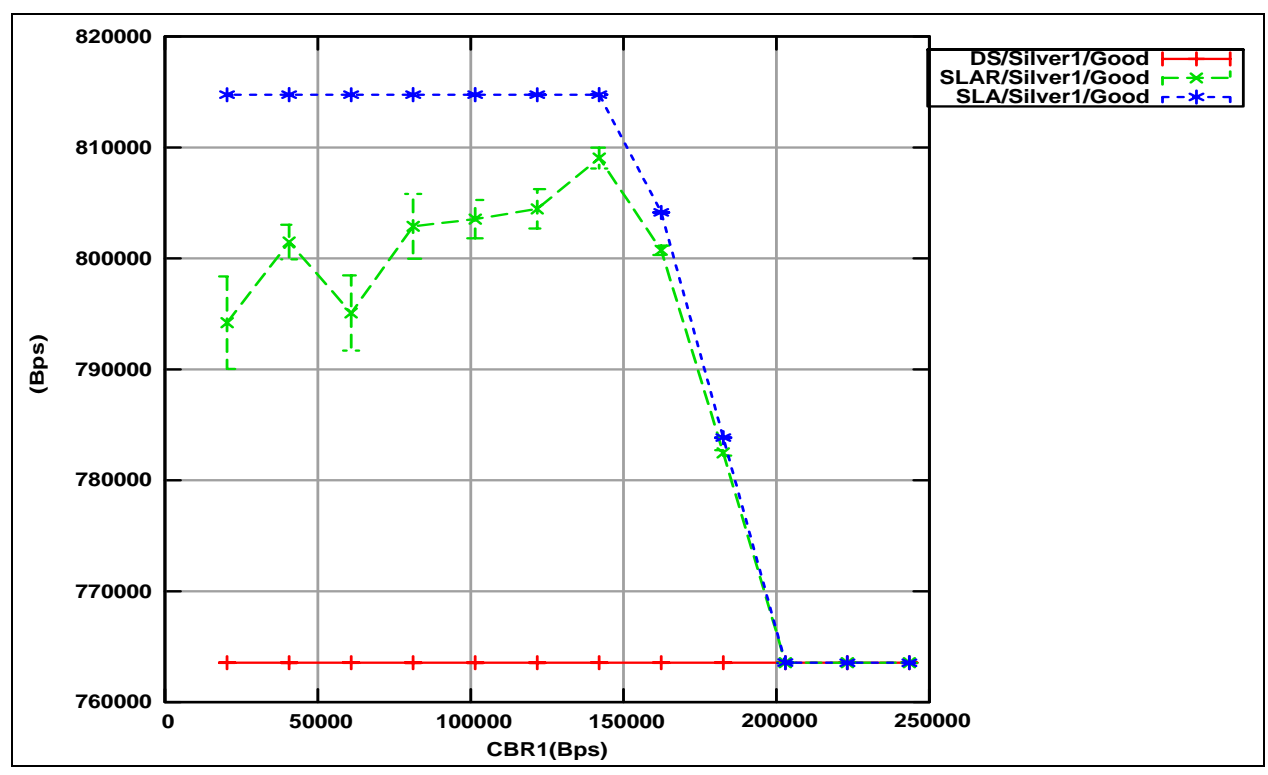

Figure 61 DS, SLAR, SLA Silver Goodput at low network load Versus Gold rate 


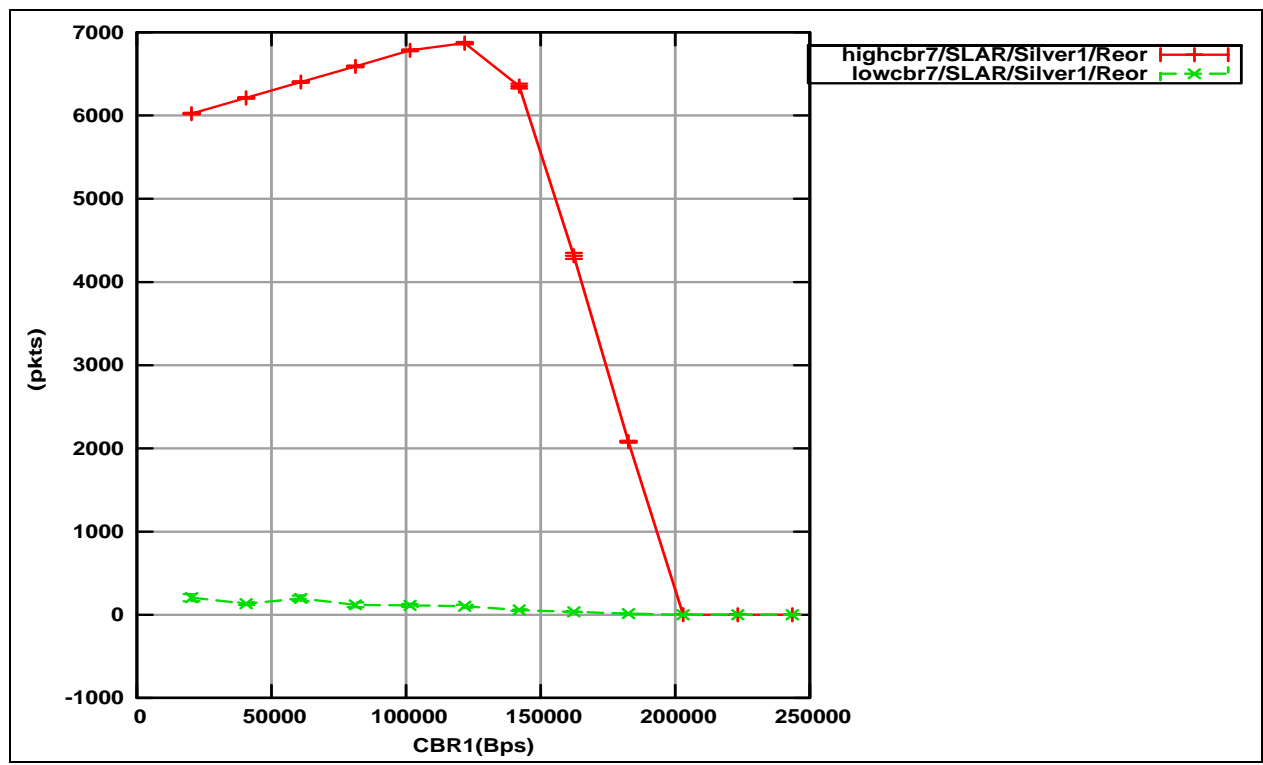

Figure 62 SLAR Reordering with high and low network load Versus Gold rate

Figure 63 shows Client1's Bronze goodput under low network load. Again compared to Figure 28 on page 94, it is obvious that the SLAR model has shown some improvement (due to little to no reordering) and that the SLA model is still better than the other 2 models. 


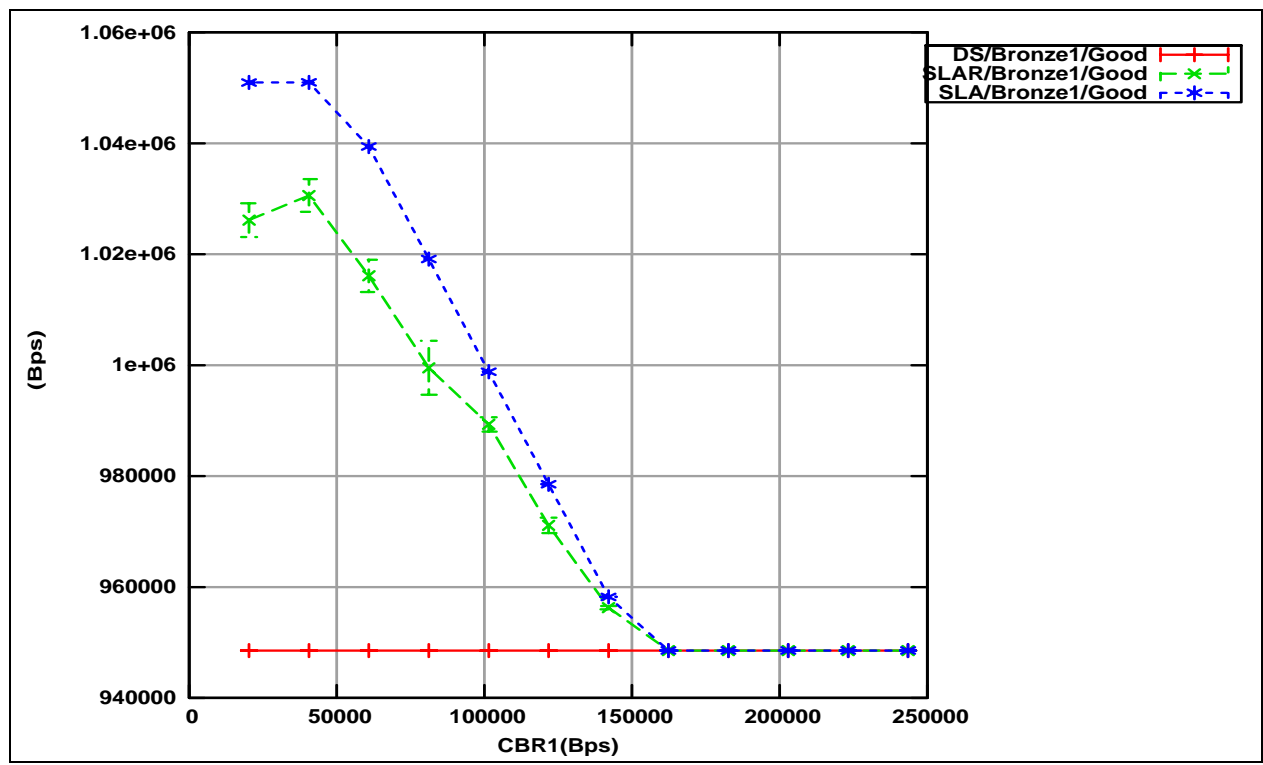

Figure 63 DS, SLAR, SLA Bronze Goodput at low network load Versus Gold rate

\subsubsection{Packet Size}

The packet size has no effect on goodput for the DS and SLA models, but has some effect on the SLAR model as shown in Figure 64 and Figure 65 (the SLA model shows a better goodput than the DS model due to upgrades). The effect on the SLAR model is clearer in Figure 65, which is explained by the various amount of reordering per packet size, as shown in Figure 66. The reordering plot shows that, as the packet size increases, the number of packets reordered decreases exponentially but does not fade away as shown for the Bronze plot (there is always a certain number of reordered packets even when the packet size grows large). Figure 65 shows that it is better to have larger packets for the same throughput, when the network shows partial reordering, since the less packets the less the chances of reordering (it requires a higher number of small packets to maintain the same throughput than large packets). 


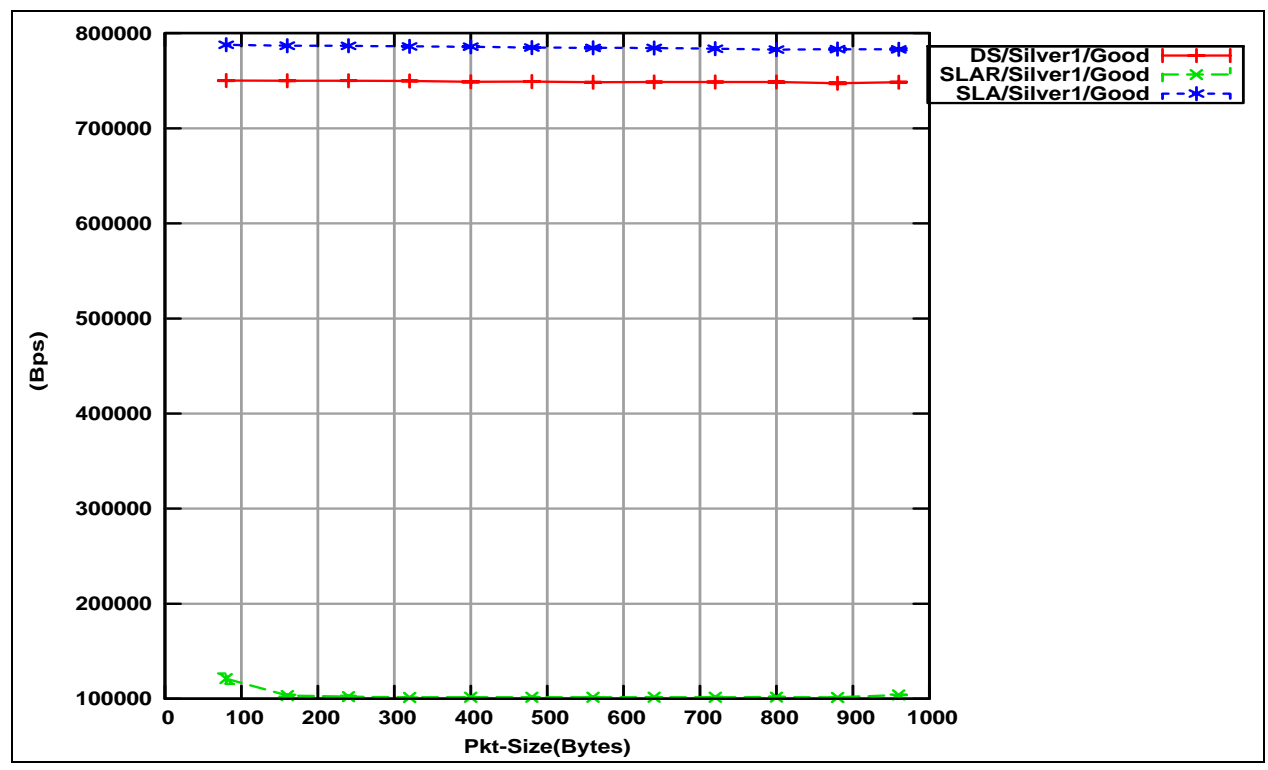

Figure 64 DS, SLAR, SLA Silver Goodput Versus Packet Size

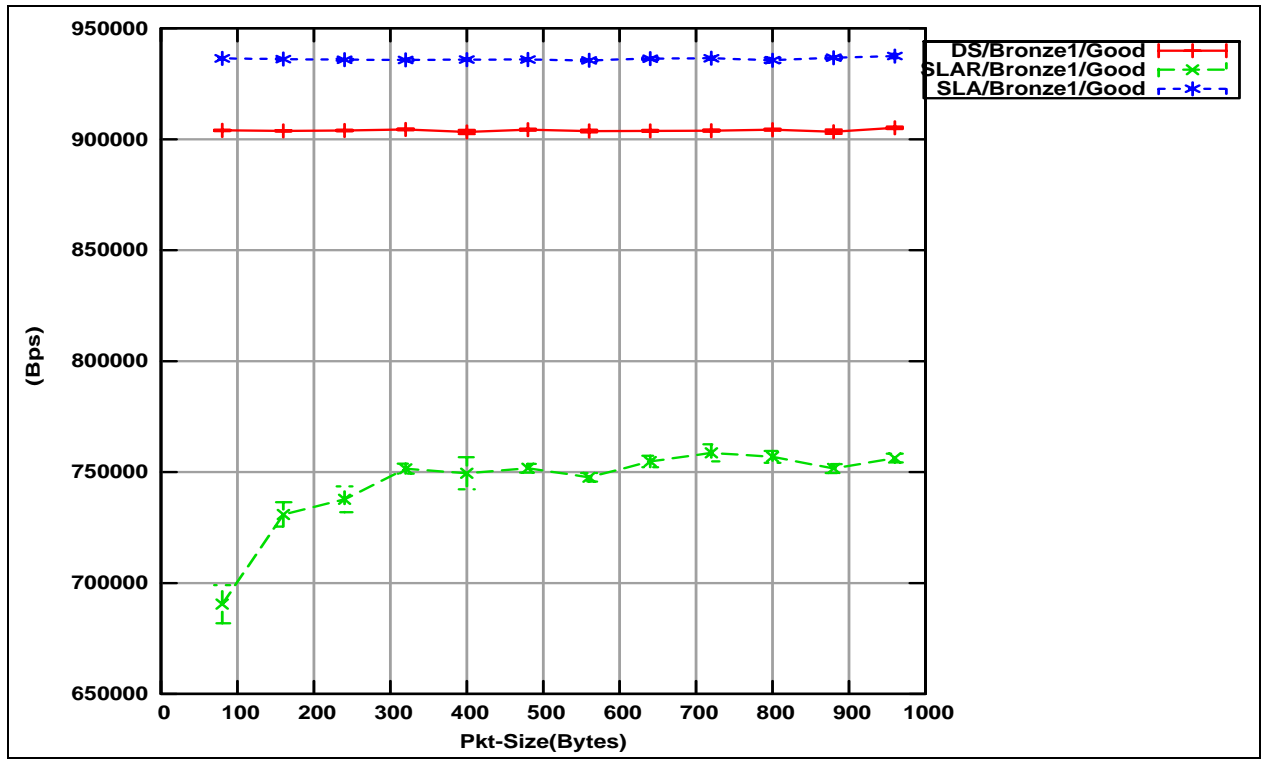

Figure 65 DS, SLAR, SLA Bronze Goodput Versus Packet Size 


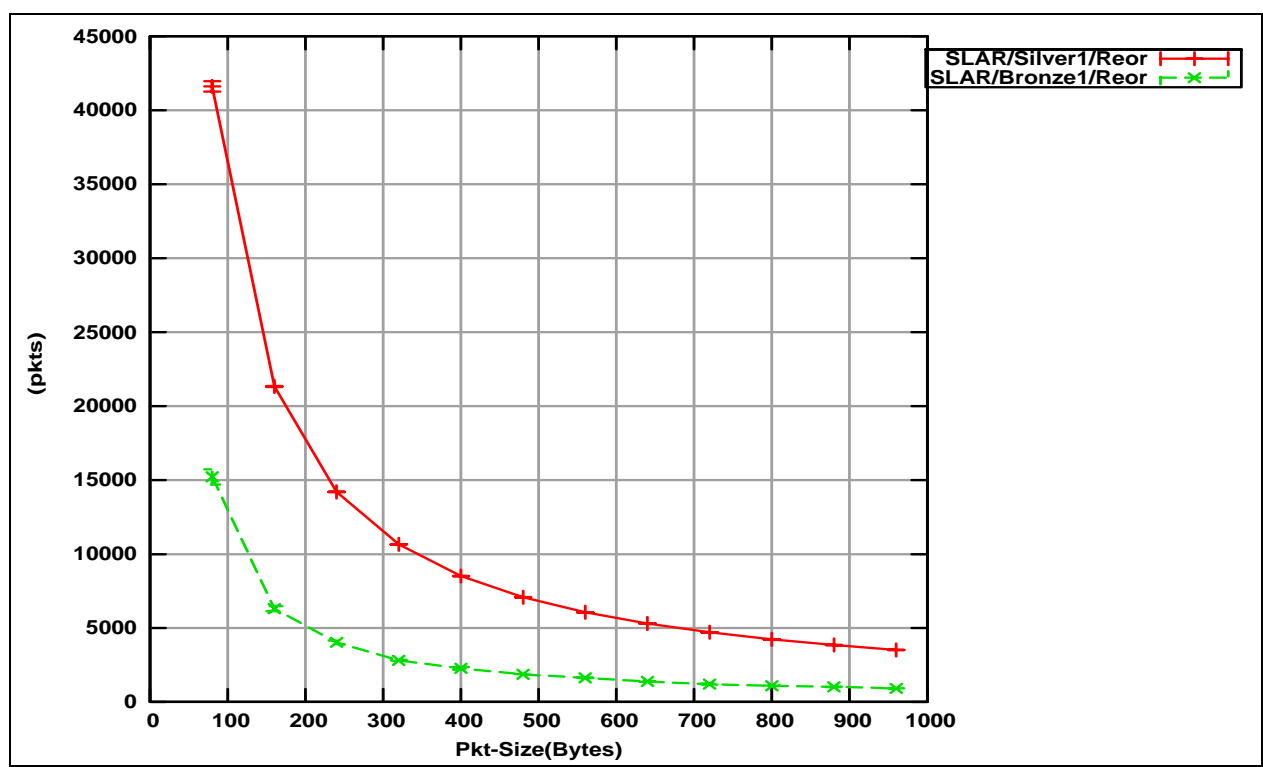

Figure 66 SLAR Reordering Versus Packet Size

Evaluating the effect of the packet size on End-to-End delay showed that the delay increases with the packet size, as shown in Figure 67. This is due to the larger transmission delays associated with larger packets. The SLAR model shows smaller "apparent" delays, first since not all the packets made it to the receiver (due to reordering), and second since the majority of the received packets where upgraded to Gold throughout the network. 


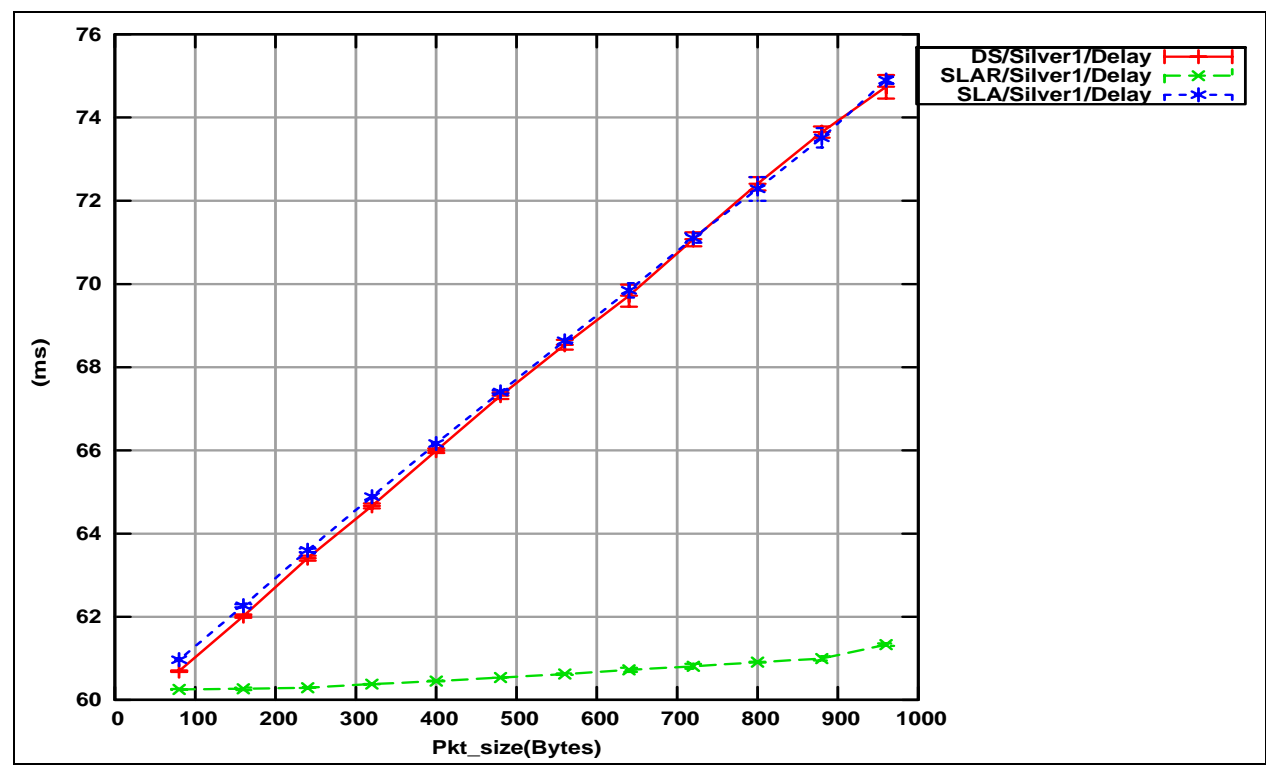

Figure 67 DS, SLAR , SLA End-to-End Silver Delay Versus Packet Size

To summarize this section's findings, we have shown that automatically upgrading packets on ingress and applying the reordering solution of section 5.4, which rectified the "packets out of order problem", achieved the desired higher goodputs at the receiver. We have demonstrated the benefits of the SLA by comparing the SLA QoS performance in terms of goodput, delay and packet losses to the DS and SLAR performances and concluded that the SLA model outperforms the other models. We have investigated the effect of the network load on reordering, and showed that the reordering is maximized when the lower class queues are filled and the upper class queues are empty. We have also established that the packet size does not affect the goodput and loss behavior of the SLA model but having larger packet sizes achieved higher goodputs in the SLAR model due to lower reordering; also, as the packet size increases, the end-to-end delay increased due to higher transmission delays. Finally, we have concluded that the SLA gives the client the ability to fully benefit from his/her higher classes reserved bandwidths without any reordering in the network. 


\subsection{Firmware Implementation}

The 3-tier SLA with automatic upgrades was implemented in firmware on Intel's 28xx family of network processors using Ericsson's Integrated Site Edge Router (ISER). The implementation is the property of Ericsson and uses Ericsson's patent-pending reordering solution. In this section we will briefly talk about various aspects of the implementation without infringing the Ericsson's intellectual property rights.

\subsubsection{Scalability}

As shown in Figure 9 on page 66, the proposed automatic upgrades solution utilizes a set of QoS components to achieve the SLA. In particular, it utilizes a couple of policers per traffic class, and separate scheduler/queues per customer. This could have some scalability issues (depending on the implementation) when the number of customers and/or traffic classes increases, since in a physical implementation resources (packet buffers, policers, etc.) are finite. However, as mentioned earlier this impacts ingress nodes only, and besides from the reordering solution there is no change in the inner nodes of the network.

\subsubsection{Tokens}

Another identified issue was the latency introduced by having the scheduler read recursively from several queues until it finds a non-token packet. For example, the scheduler might have to recursively read and remove tokens from all queues (if a token was found in each of the queues except the last queue corresponding to the best effort or least priority) until it finds a non-token or valid packet. If the number of priority classes increases, this latency can affect the ability to do wire-speed processing. 


\subsubsection{Policers in series}

Finally, one more identified issue was the latency introduced by the number of policers in series when upgrading a packet. In the worst case (this does not depend on the number of priority classes), a packet might have to be policed three times before a decision on whether to forward the packet or drop it if it can not be upgraded. As mentioned earlier, this could affect the ability to do wire-speed processing.

\subsection{Summary Observations}

In this chapter, we focused on the analysis of the 3-tier SLA with automatic upgrades and investigated the 3 models' (DS, SLAR, SLA) differences in terms of QoS performance like throughput, goodput, delay and loss. We showed that the SLAR model can not compete with the other 2 models due to packet reordering which is interpreted as loss on the receiver side, especially when the receiver does not buffer out of order packets. We observed that the SLA model outperformed both the DS and the SLAR models in showing higher throughput, higher goodput, and lower losses by allowing customer lower class packets to fill the upper classes rate gaps. We also argued that the SLA introduced delays due to class upgrades falls well within the DS maximum rate delays and should be within the acceptable promised classes' delays. Then, we showed that the SLA provides better service than the other 2 models for customers regardless of the network load, and is significantly more useful when the network load is low. We also showed that the SLA behavior does not depend on the packet size. Finally, we highlighted possible scalability and latency issues with the proposed implementation of the automatic upgrades SLA based on Intel's 28xx Network Processor firmware implementation using Ericsson's ISER edge router. 


\section{Chapter 7}

\section{Experimental Results - Bursty Traffic}

In Chapter 6, we analyzed the performance of the SLA mainly under constant rate traffic, in order to highlight the benefit of upgrading packets to fill up rate gaps in higher classes. We have shown that the SLA outperforms the DS and the SLAR models in providing clients better goodput and lower loss with "within-the-norm" delays; customers get what they paid for and what they expect, for the leased pipes of various classes of service (fully use the paid for bandwidths).

In this chapter, we will investigate the performance of the proposed SLA under bursty traffic. For that matter, we will use the Interrupted Poisson Process (IPP), as described in $[83,84]$, as an arrival process. The IPP is an ON/OFF process, where both the ON and the OFF periods are exponentially distributed. We will also use a higher-speed network in order to capture the benefit of the SLA on the boundary between two providers where not only the link speeds are expected to be higher but also the SLA rates (we mentioned earlier that the SLA could be a contract between a client and a provider, and also between two providers; we 
also mentioned that the benefit on a providers' boundary should be higher since the SLA rates are expected to be higher).

We first describe in section 7.1 the IPP arrival process in more detail along with the changes in the simulation configuration from the previous chapter, which includes using OC3-speed links with lower propagation delay. In section 7.2 we present the results and analyze the behavior of the SLA in this scenario, and finally we summarize the findings of the analysis in section 7.3.

\subsection{Simulation Configuration}

In this scenario, we target the analysis of a provider-provider boundary where the links are of higher speeds (OC3 or 155.54 Mbps or 19.44 MBps), and we use a total propagation delay equal to the estimated US East-to-West coast propagation delay of $30 \mathrm{~ms}$ (so the propagation delay is $5 \mathrm{~ms}$ per link). The network topology is the same, as shown in Figure 15 on page 78.

We assume that the provider-provider traffic is bursty in nature, and we capture the burstiness using an IPP arrival process. The IPP arrival process is an ON/OFF process, where both the ON and the OFF are exponentially distributed. The squared coefficient of variation, $\mathrm{c}^{2}$, of the packet inter-arrival time characterizes the burstiness of the packet arrival process $[83,84]$. This coefficient is defined as the variance of the packet inter-arrival time divided by the squared mean packet inter-arrival time. The higher the coefficient of variation value, the higher the packet arrival burstiness $\left(c^{2}=19\right.$ emulates a compressed voice conversation where the silence is suppressed). 
Assuming the ON period distribution is exponential with average $1 / \mu_{1}$ and the OFF period distribution is exponential with average $1 / \mu_{2}$, we have:

$$
c^{2}=1+\frac{2 \lambda \mu 1}{(\mu 1+\mu 2)^{2}}
$$

where $\lambda$ is the arrival rate of a packet during the ON period $(1 / \lambda=$ packet size / Link capacity).

The average arrival rate is the average number of bytes that has arrived during an ON/OFF cycle. So assuming that packets arrive back to back within the ON period, the arrival process is characterized by

$$
\text { averagearrival rate }=\frac{(\operatorname{Link} \operatorname{Cap}) \mu 2}{\mu 1+\mu 2}
$$

Thus, knowing the link capacity (Link_Cap), 155.54Mbps in our case, also knowing the desired average arrival rate, and the $c^{2}$ value, we can derive $\mu_{1}$ and $\mu_{2}$. Finally, in our simulations we assume the packet length is exponentially distributed with an average of 500 bytes (the last packet of the ON period may be truncated so that its last bit arrives at the end of the ON period).

Table 8 and Table 9 summarize the various parameters and values used in this scenario (the various parameters' definitions were explained in section 6.2.2). 
Table 8 Network Parameters, Scenario 2

\begin{tabular}{|l|l|l|l|l|l|l|l|l|l|}
\hline Client & Class & Traffic type & $\begin{array}{l}\text { Rate } \\
\text { (Bps) }\end{array}$ & $\begin{array}{l}\text { Pkt Size } \\
\text { (B) }\end{array}$ & $\begin{array}{l}\text { LB CBS } \\
\text { (B) }\end{array}$ & $\begin{array}{l}\text { LB CIR } \\
\text { (Bps) }\end{array}$ & $\begin{array}{l}\text { FS } \\
\text { WRR }\end{array}$ & $\begin{array}{l}\text { SS } \\
\text { WRR }\end{array}$ & GSR \\
\hline Client1 & Gold & UDP IPP & 202915 & 500 & 50000 & 505830 & 106 & 269 & 554312 \\
\hline Client1 & Silver & UDP IPP & 1629508 & 500 & 50000 & 1427108 & 399 & 269 & 2086514 \\
\hline Client1 & Bronze & UDP IPP & 2101908 & 500 & 50000 & 1797108 & 495 & 269 & 2588533 \\
\hline & & & & & & & & & \\
\hline Client2 & Gold & TCP FTP & Dynamic & $<1200$ & 50000 & 307200 & 25 & 65 & 421200 \\
\hline Client2 & Silver & TCP FTP & Dynamic & $<1200$ & 50000 & 307200 & 25 & 65 & 421200 \\
\hline Client2 & Bronze & TCP FTP & Dynamic & $<1200$ & 50000 & 307200 & 25 & 65 & 421200 \\
\hline & & & & & & & & & \\
\hline Client3 & Gold & UDP IPP & 426063 & 500 & 50000 & 1426062 & 100 & 100 & 1944000 \\
\hline & & & & & & & & & \\
\hline Client4 & Silver & UDP IPP & 3668616 & 500 & 100000 & 5502924 & 100 & 257 & 4996080 \\
\hline & & & & & & & & & \\
\hline Client5 & Bronze & UDP IPP & 4408617 & 500 & 100000 & 6612924 & 100 & 309 & 6006960 \\
\hline & & & & & & & & & \\
\hline bg1 & Gold & UDP CBR & 534773 & 500 & & & & & \\
\hline bg1 & Silver & UDP CBR & 2751462 & 500 & & & & & \\
\hline bg1 & Bronze & UDP CBR & 4959694 & 500 & & & & & \\
\hline & & & & & & & & & \\
\hline bg2 & Gold & UDP CBR & 534773 & 500 & & & & & \\
\hline bg2 & Silver & UDP CBR & 2751462 & 500 & & & & & \\
\hline bg2 & Bronze & UDP CBR & 4959694 & 500 & & & & & \\
\hline & & & & & & & & & \\
\hline bg3 & Gold & UDP CBR & 534773 & 500 & & & & & \\
\hline bg3 & Silver & UDP CBR & 2751462 & 500 & & & & & \\
\hline bg3 & Bronze & UDP CBR & 4959694 & 500 & & & & & \\
\hline
\end{tabular}


Table 9 Nodes Configuration, Scenario 2

\begin{tabular}{|l|l|l|l|l|l|l|}
\hline Node & Class & RED A & RED B & RED P & WRR & GSR \\
\hline n2n3 & Gold & inf & inf & 0 & 17 & 3304800 \\
\hline n2n3 & Silver & 25 & 35 & 0.5 & 38 & 7387200 \\
\hline n2n3 & Bronze & 35 & 45 & 0.7 & 45 & 8748000 \\
\hline & & & & & & \\
\hline n3n4 & Gold & inf & inf & 0 & 17 & 3304800 \\
\hline n3n4 & Silver & 25 & 35 & 0.5 & 38 & 7387200 \\
\hline n3n4 & Bronze & 35 & 45 & 0.7 & 45 & 8748000 \\
\hline & & & & & & \\
\hline n4n5 & Gold & inf & inf & 0 & 17 & 3304800 \\
\hline n3n4 & Silver & 25 & 35 & 0.5 & 38 & 7387200 \\
\hline n3n4 & Bronze & 35 & 45 & 0.7 & 45 & 8748000 \\
\hline
\end{tabular}

Notice in this scenario, since we are using bursty sources (IPP), the CBS (Committed Burst Size) values are relatively larger ( 50,000 or 100,000 bytes) than the CBR case of the previous chapter (we used $\mathrm{CBS}=5000$ for $\mathrm{CBR}$ sources). Moreover, since the End-to-End background traffic is also bursty, we use relatively higher background traffic rates to emulate the provider's bandwidth multiplexing to keep the network utilization high (in fact, providers usually overbook their reserved traffic classes with some statistical analysis of the clients' traffic types in order to fully use the network capacity; their revenue depends on the number of clients they can sell service to). Finally, we use higher RED configuration with average queue sizes of $\min =25, \max =35$ for Silver and $\min =35, \max =45$ for Bronze (compared to $\min =20, \max =30$ for Silver and $\min =30, \max =40$ for Bronze in the previous chapter). This is intended to relax the drop ratio in the RED buffer management algorithm in order to accommodate a high variance in the queue sizes within the network due to the usage of a relatively high $\mathrm{c}^{2}$ (the higher the coefficient of variation value, the higher the packet arrival burstiness). 


\subsection{Results Analysis}

In this section we present the simulations results along with the analysis of the behavior of the DS, SLAR and SLA models. We first show the IPP arrivals in section 7.2.1 in order to display the burstiness of the arrival process, then we talk about the various QoS performance metrics starting with throughput and goodput in section 7.2.2, then packet loss in section 7.2.3, and finally the end-to-end delay in section 7.2.4.

\subsubsection{IPP arrivals}

As mentioned in section 7.1, the IPP average arrival rate is in function of both the ON and OFF average periods. We plot, in Figure 68, 150 seconds of the Gold traffic arrival rate with $1 / \mu_{1}=0.000295696,1 / \mu_{2}=0.023655735$ and link_cap $=19.44 \mathrm{Mbps}$, based on a 0.1 second moving window average (each point in the graph is the number of bytes received at the first hop within a 0.1 second interval). Figure 69 shows the same information zooming in on the first 20 seconds. The plots show the high burstiness of the traffic, given a computed average of $\sim 240 \mathrm{KBps}$ (the squared coefficient of variation, $\mathrm{c}^{2}$, of the packet inter-arrival time was set to 20). 


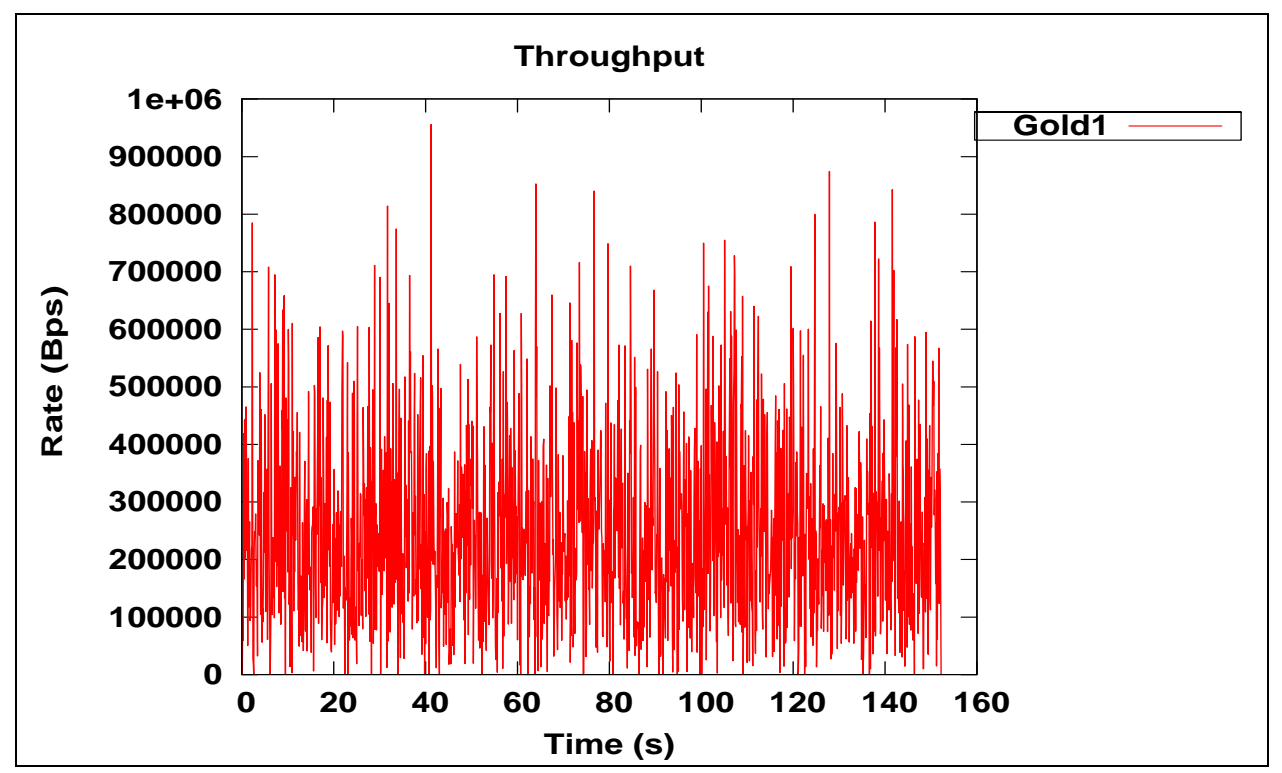

Figure 68 IPP arrival (150 second), $1 / \mu_{1}=0.000295696$

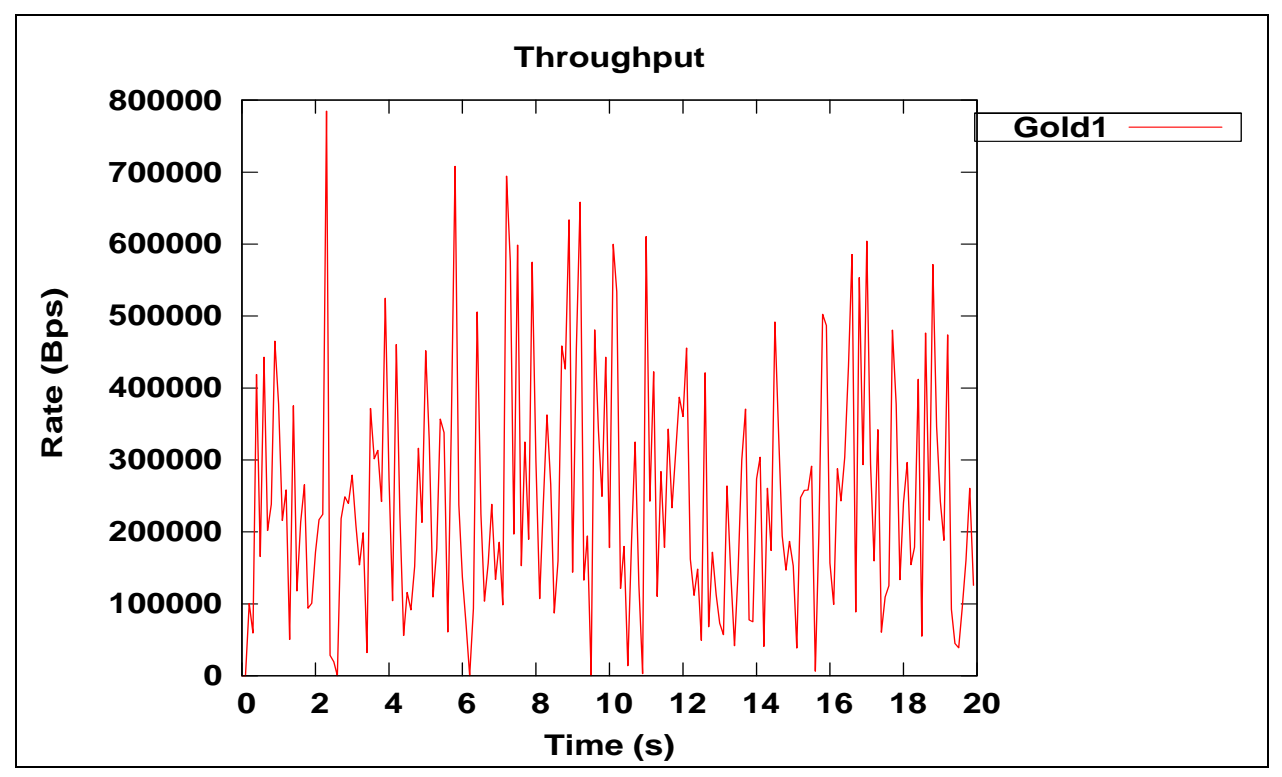

Figure 69 IPP arrival (20 second), $1 / \mu 1=0.000295696$

Figure 70 shows the difference of the Gold IPP traffic when varying $1 / \mu_{1}$, for $1 / \mu_{1}=$ 0.0005988785 (with an average arrival rate of $\sim 480 \mathrm{KBps}$ ) and $1 / \mu_{1}=0.000295696$ (with an average arrival rate of $\sim 240 \mathrm{KBps})$. 


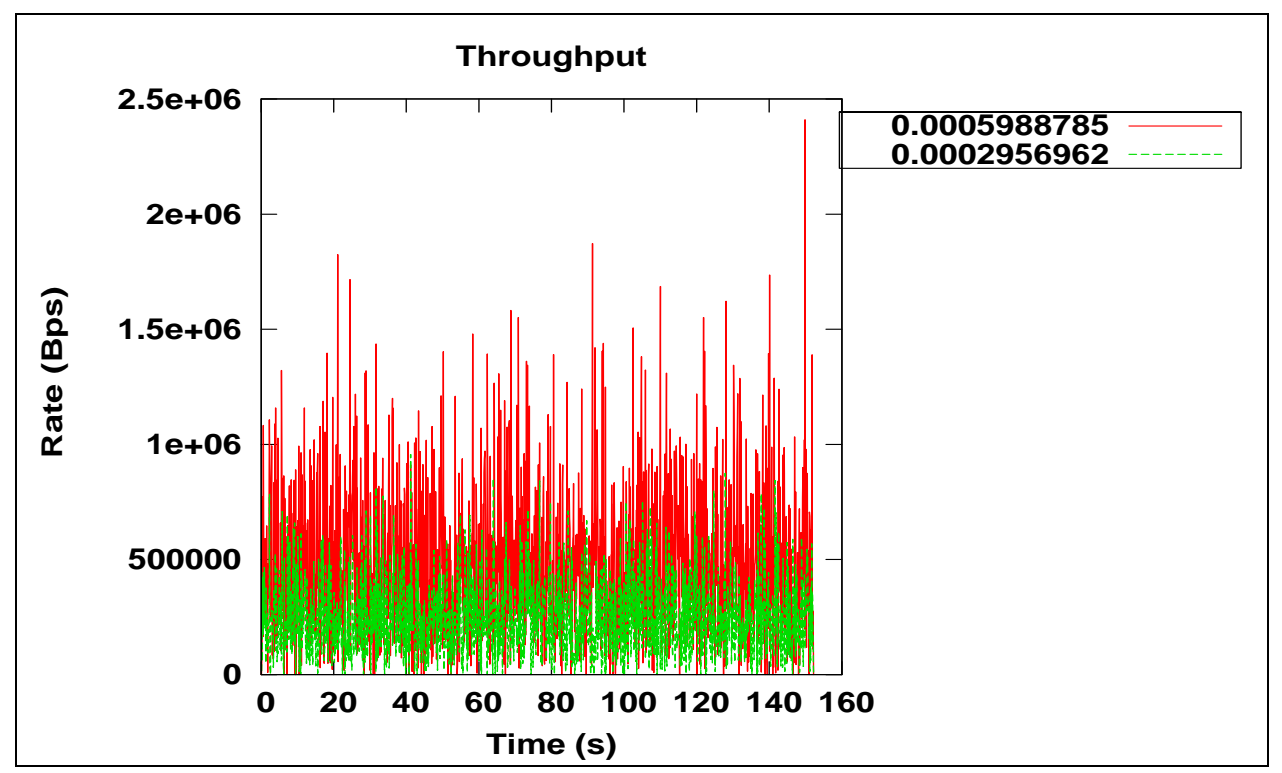

Figure 70 IPP arrivals with different average rates

Figure 71 shows the probability density functions (PDFs) for both the ON and OFF periods for $1 / \mu_{1}=0.000295696,1 / \mu_{2}=0.023655735$. And finally, Figure 72 shows the PDF for the packet size during the ON period (the average packet size is 500 Bytes).

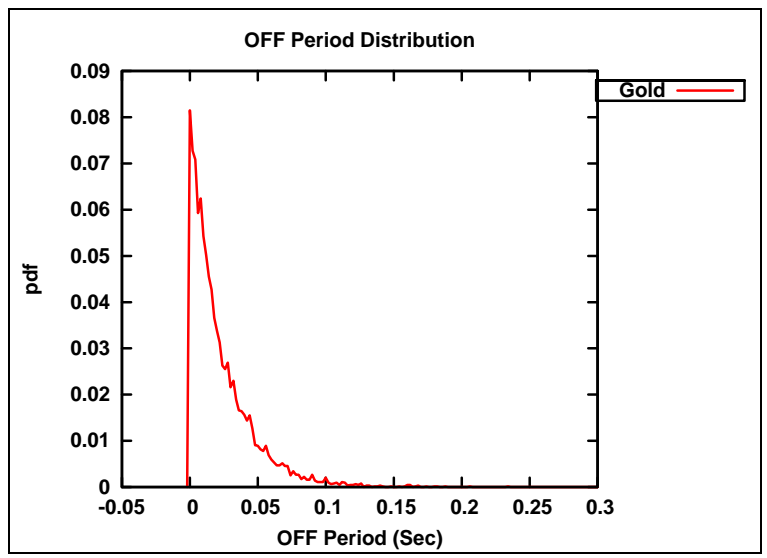

Figure 71 ON and OFF distributions for $1 / \mu_{1}=0.000295696,1 / \mu_{2}=0.023655735$ 


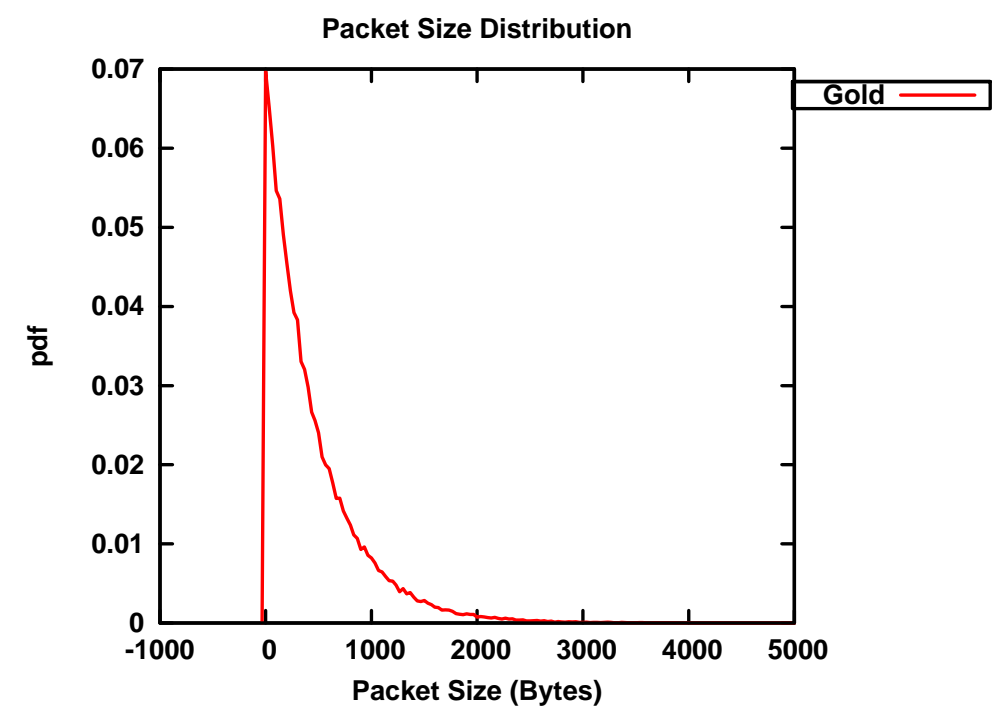

Figure 72 Packet Size distribution during the ON period (average $=500$ Bytes)

\subsubsection{Throughput and goodput}

In the following figures, unless otherwise stated, we will vary Client1's Gold traffic rate by varying the ON period exponential distribution average $1 / \mu_{1}$ and fixing the OFF period exponential distribution average to $1 / \mu_{2}=0.023655735$. Figure 73 shows Client1's average Gold throughput measured at the first ingress node for all 3 models as a function of $1 / \mu_{1}$. Remember from section 7.1 that the average arrival rate is given by:

$$
\text { averagearrival rate }=\frac{(\operatorname{Link} \operatorname{Cap}) \mu 2}{\mu 1+\mu 2}
$$

Thus, for example, for a link capacity of $19.44 \mathrm{MBps}, 1 / \mu_{1}=0.000295696$ and $1 / \mu_{2}=$ 0.023655735 , Client1's Gold average rate is computed to be $\sim 240 \mathrm{KBps}$ (very close to the measured throughput as shown in the figure). The small difference in throughput for the 3 models shown in the figure is due to the randomness of the arrivals (IPP process). 


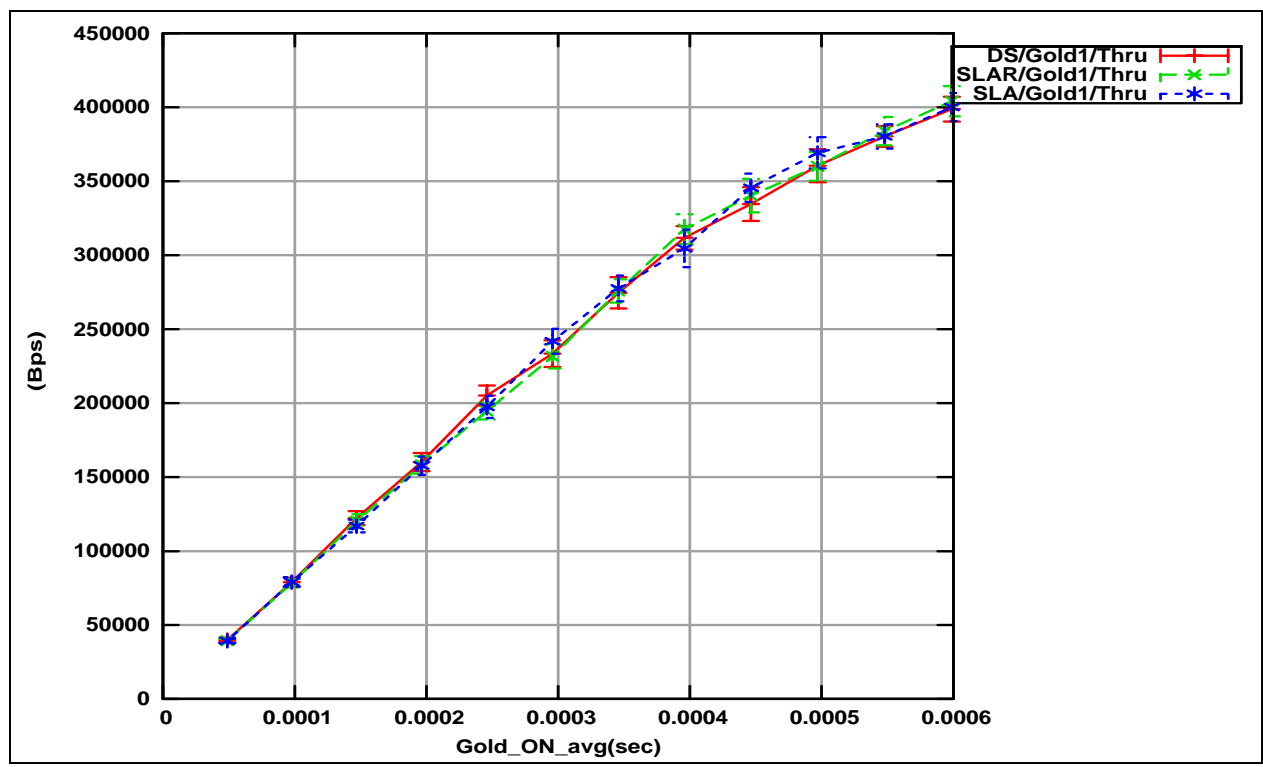

Figure 73 Client1 Gold Throughput Versus Gold's ON period $\left(1 / \mu_{1}\right)$

First we start by displaying in Figure 74 and Figure 75 the throughput measured at the first node (ingress node) of Client1's Silver and Bronze traffic versus the Gold ON period (or rate) of each of the DS, SLAR, and SLA models.

Figure 74 shows that as Client1's Gold rate increases (by increasing the average Gold ON period) the DS Silver throughput is not affected since the DS Silver traffic is policed independently from the Gold traffic, whereas the SLAR and the SLA models allowed extra Silver traffic to fill the gap in the Gold allowed bandwidth (from Table 8 the Gold CIR is 505,830 Bps). Figure 76 shows the rate of Client1's Silver upgraded packets (in Bps) as the Gold traffic rate increases. The figure indicates that the Silver traffic was upgraded to fill the gap created by the Gold rate since the sum of the Silver upgrade rate and the Gold rate is $\sim 500 \mathrm{KBps}$ (the configured Gold policed rate).

Figure 75 shows that as the Gold rate increases (by increasing the ON period), the DS model Silver and Bronze rates are not affected since they are policed independently and the DS model does not upgrade lower class packets to fill upper class gaps. On the other hand, 
for the SLAR and the SLA models, since they do allow upgrades, as the Gold traffic rate increases the less the Silver upgrades and thus the less the Silver throughput. The less the Silver upgrades means that the non-upgraded Silver packets have to find room in the Silver rate otherwise dropped. This forces upgraded Bronze packets to be pushed back to Bronze (i.e. not upgraded to Silver) and thus force non-conformant Bronze packets to be dropped. In other words, as the Gold rate increases, the smaller the Gold gap, the smaller the Silver upgrades and the lower the Silver throughput (due to Silver policing). As the Gold rate continues to increase, the Silver rate is less and less upgraded and the Silver gap becomes smaller, since the Silver traffic has to find Silver bandwidth for it to pass the ingress policing. The lower the Silver gap, the smaller the Bronze upgrades and thus the smaller the Bronze throughput. This is verified in Figure 77 where we can see that the Bronze upgrades continue to drop as the Silver refits itself in Silver bandwidth (the Silver throughput, which is composed of Silver upgrades and Silver non-upgrades, decreases due to Silver policing at 1,427,108 Bps as shown in Table 8).

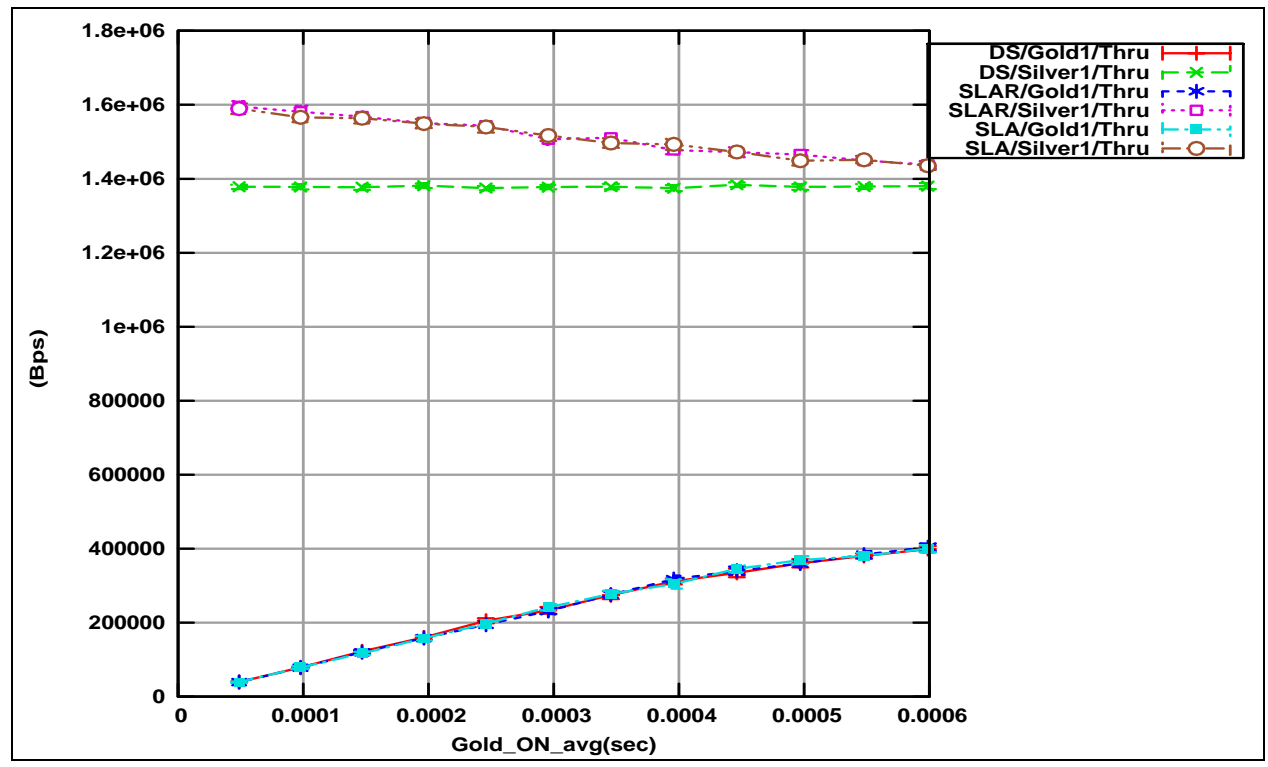

Figure 74 Client1 Gold and Silver Throughput Versus Gold's ON period $\left(1 / \mu_{1}\right)$ 


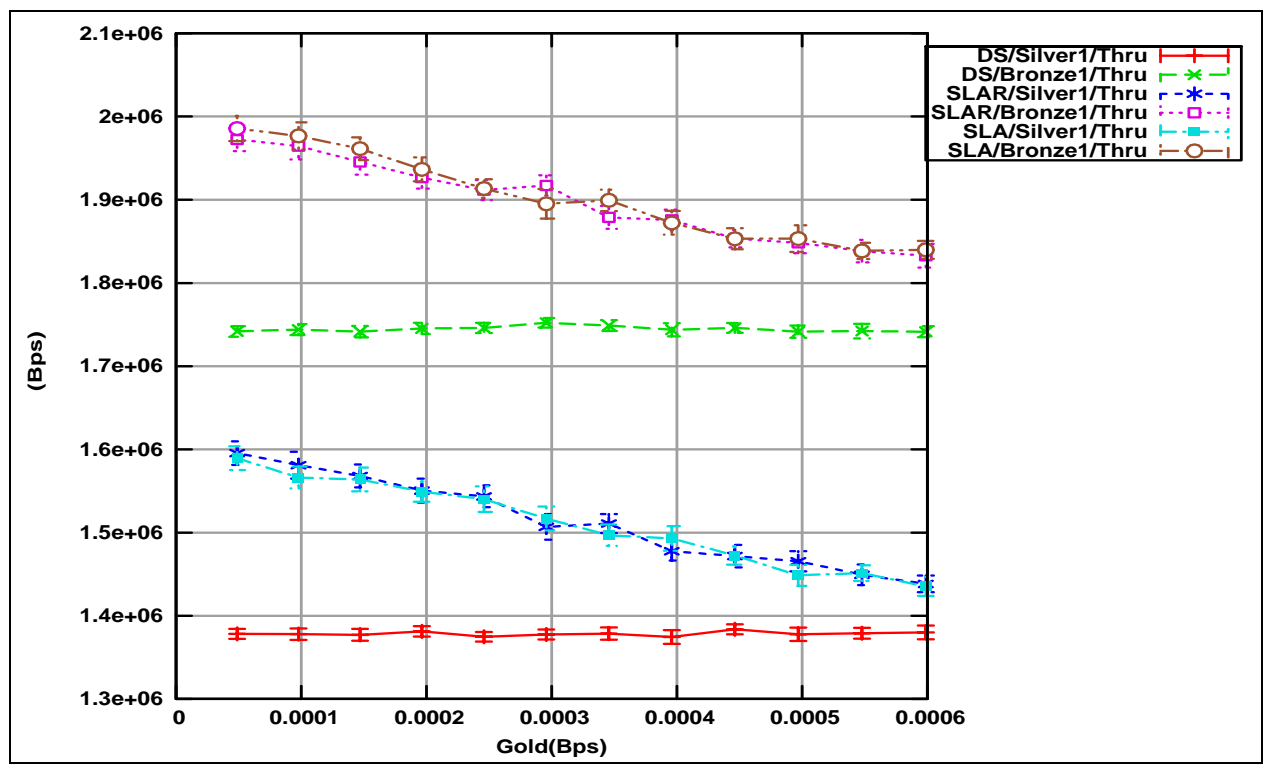

Figure 75 Client1 Silver and Bronze Throughput Versus Gold's ON period $\left(1 / \mu_{1}\right)$

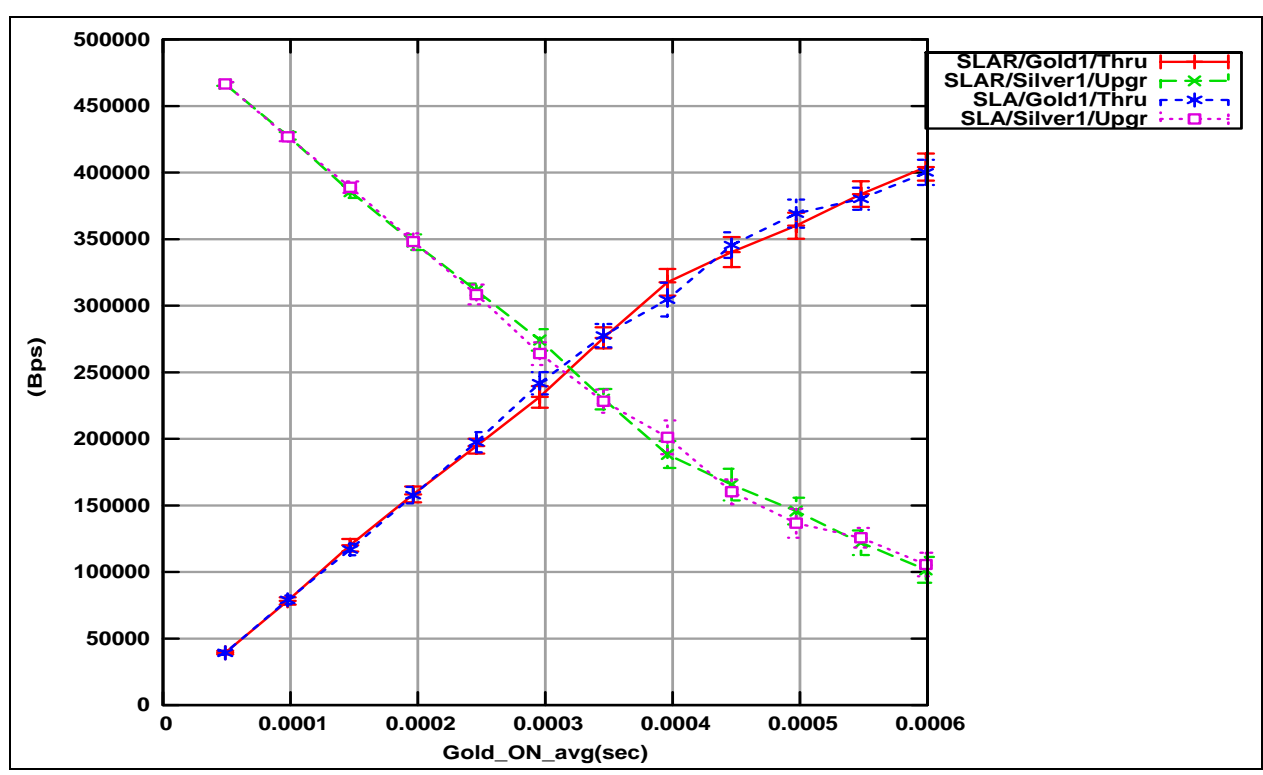

Figure 76 Client1 Gold Throughput and Silver Upgrades Versus Gold's ON period $\left(1 / \mu_{1}\right)$ 


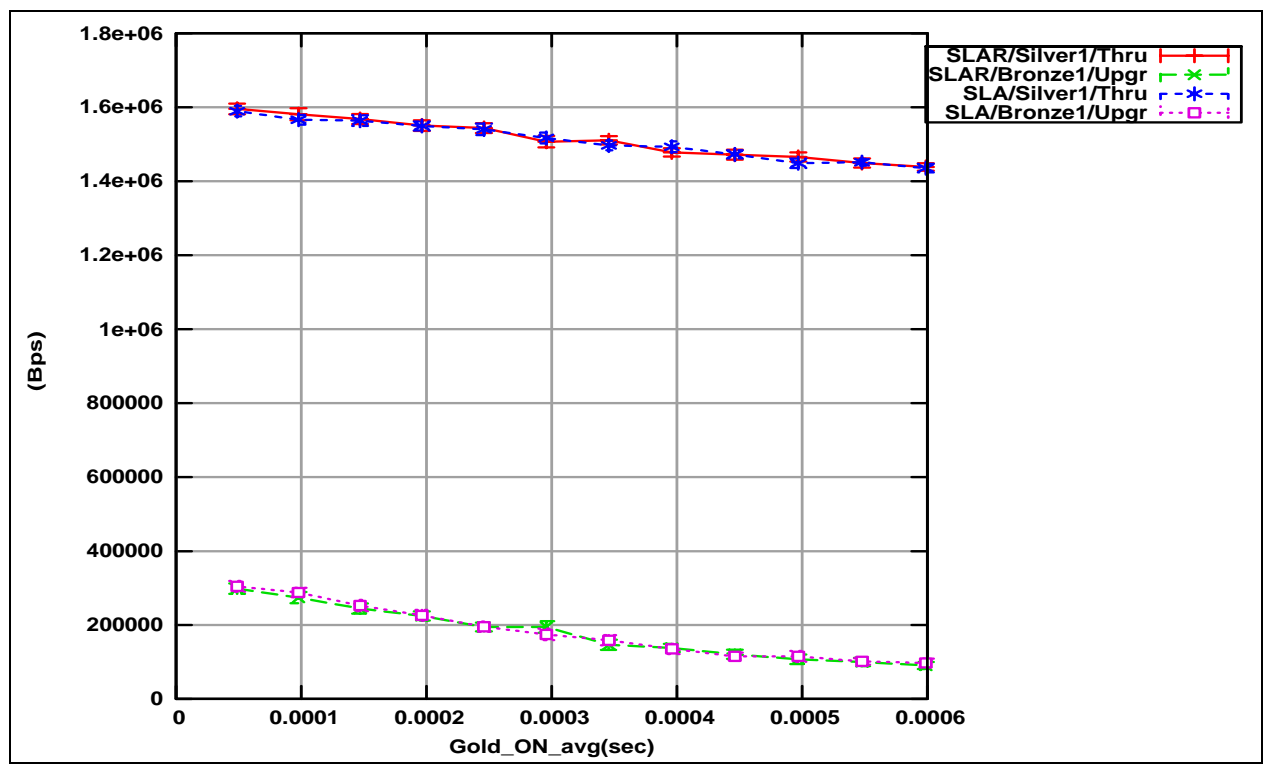

Figure 77 Client1 Silver Throughput and Bronze Upgrades Versus Gold's ON period $\left(1 / \mu_{1}\right)$

Even though Figure 75 shows that the SLAR and the SLA models have an advantage over the DS model by accepting more customer traffic into the network and thus offering to the customer the full rates (especially the expensive ones) that $\mathrm{s} /$ he paid for, plotting the goodput measured at the receiver shows that the SLAR model is not as promising, but the SLA model on the other hand outperformed the other 2 models.

Figure 78 shows Client1's Silver Goodput for the 3 models versus the Gold rate. As can be seen in the figure, the SLAR model showed really low goodput for the highest SLAR throughput (remember from Figure 75 that the Silver SLAR throughput was the highest when the Gold rate was the lowest). This is explained by the amount of reordered Silver packets in the network, which are considered as lost at the receiver (the number of SLAR reordered Silver packets versus Gold rate is shown in Figure 80).

Client1's Bronze goodput for the 3 models is shown in Figure 79. Again, the SLA model outperformed the other 2 models due to packet upgrades and no reordering in the 
network, and the SLAR model showed low goodput due to packet reordering (as shown in Figure 80).

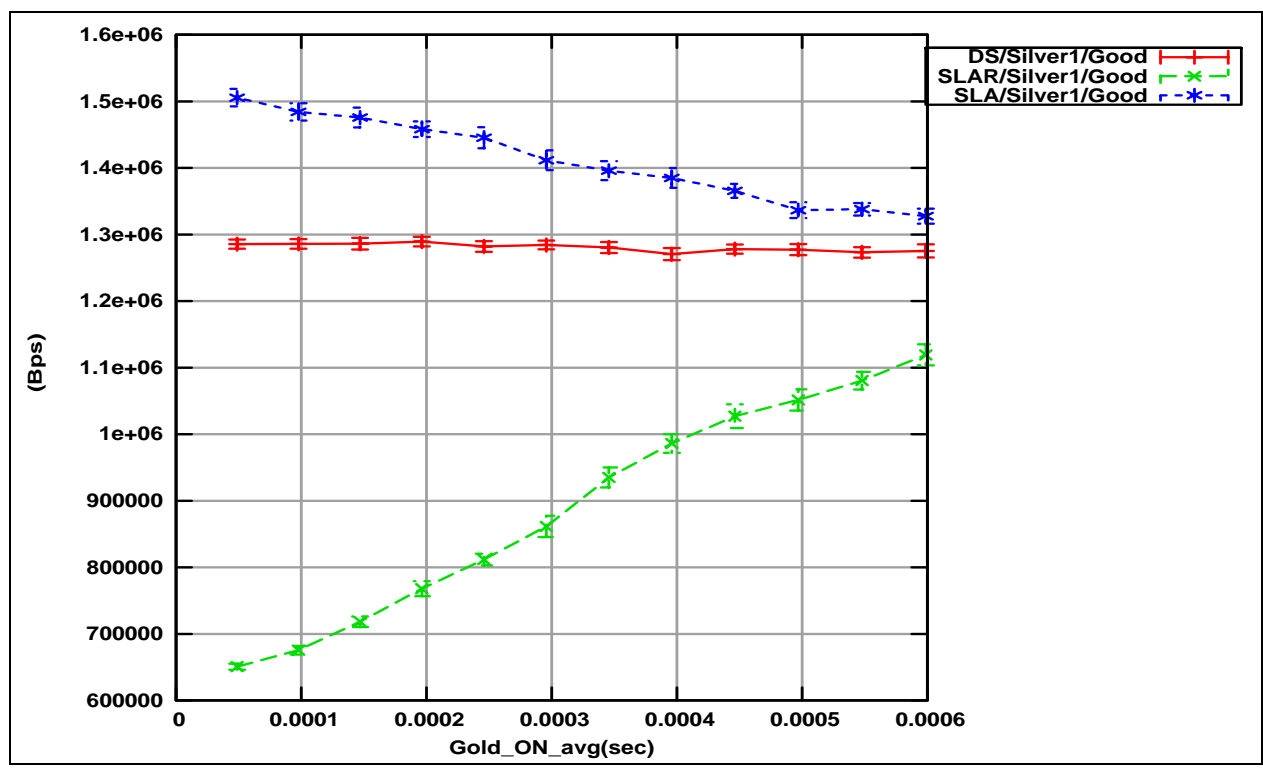

Figure 78 Client1 Silver goodput Versus Gold's ON period $\left(1 / \mu_{1}\right)$

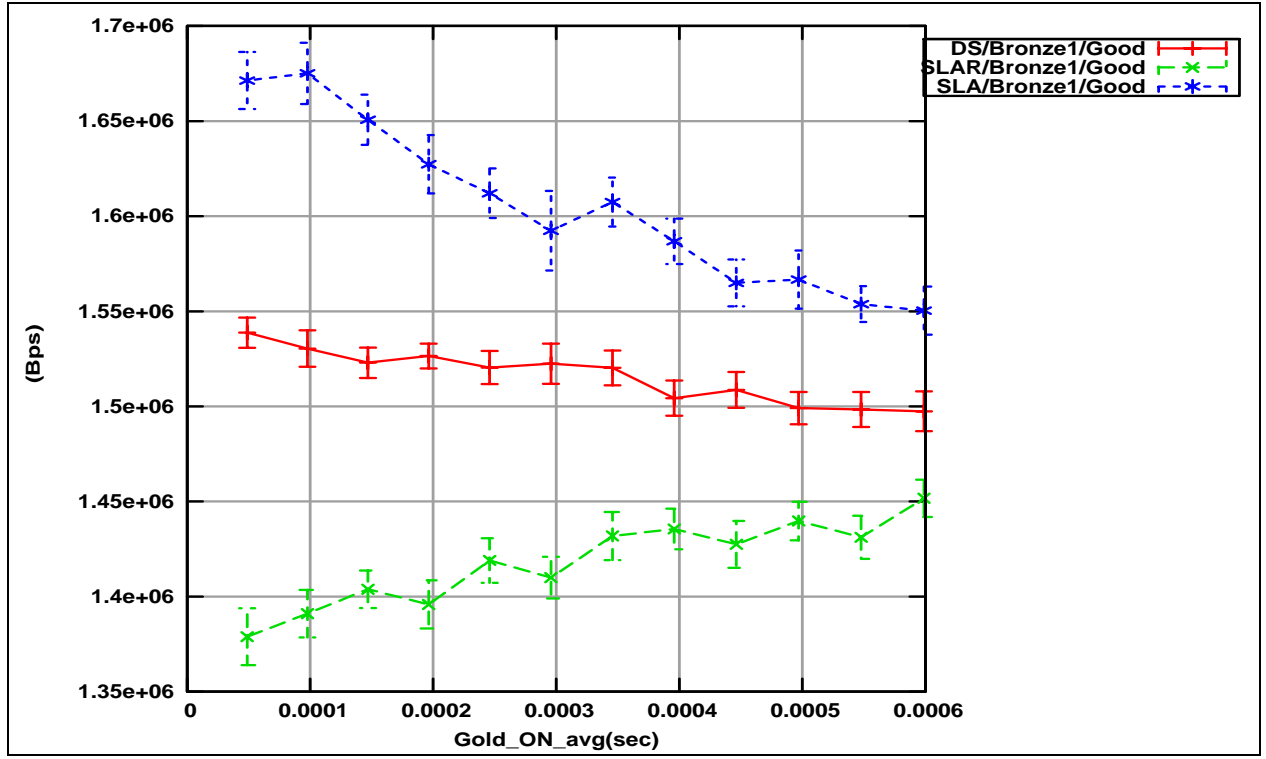

Figure 79 Client1 Bronze goodput Versus Gold's ON period $\left(1 / \mu_{1}\right)$ 


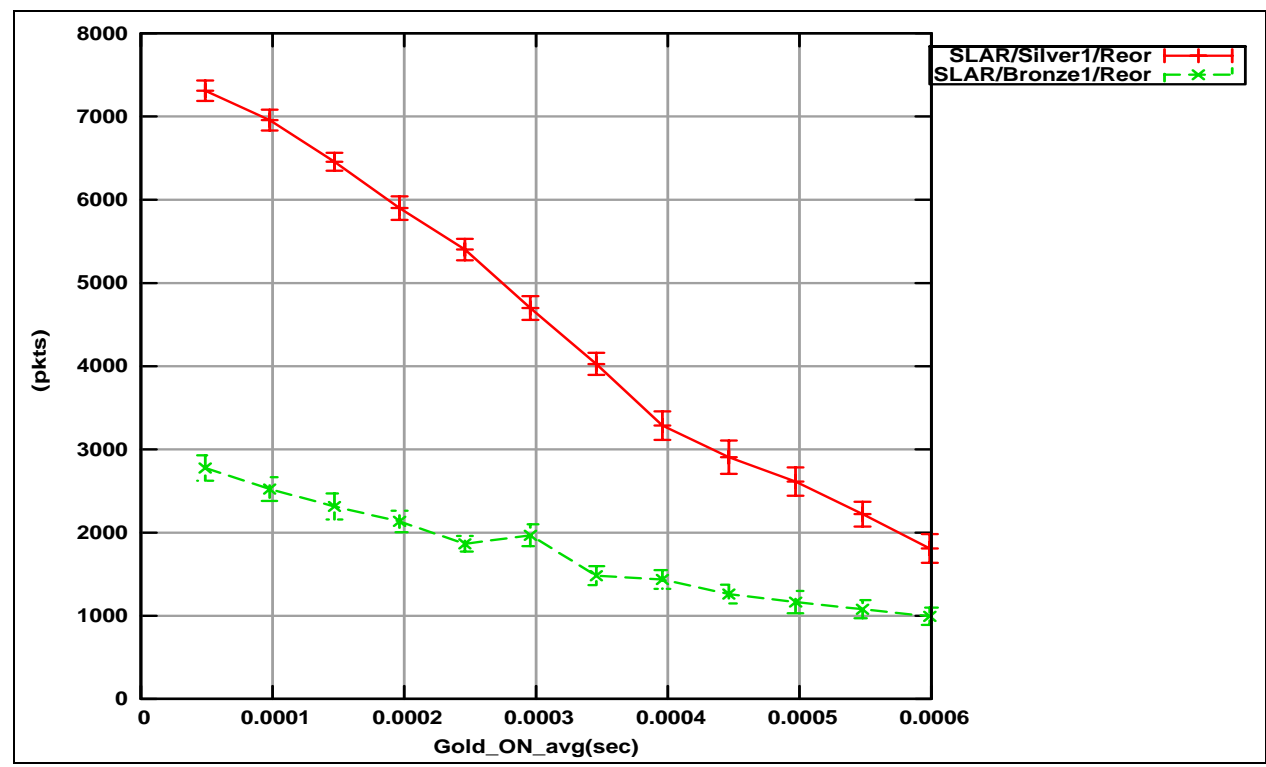

Figure 80 Client1 Silver and Bronze reordering Versus Gold's ON period $\left(1 / \mu_{1}\right)$

The reason for a decreasing amount of reordered packets as the Gold traffic increases was explained in section 6.4.2.1. Briefly, we mentioned in that section that

for reordering to occur, upgraded packets need to bypass non-upgraded packets in the network. The probability for an upgraded packet to bypass non-upgraded packets is the highest when the upgraded packet observes low queuing in the network and that the nonupgraded packets observe high queuing. So, the highest probability of reordering occurs when the Gold queue size is small compared to the Silver queue size. In other words, we expect higher number of packet reordering when the Gold queue has a relatively smaller size than the Silver queue. With IPP arrivals, increasing the Gold rate increases the average Gold queue sizes in the network while keeping the Silver queues sizes relatively the same (since we are not changing the Silver rate). With larger Gold queue sizes, the upgraded Silver packets observe higher queuing delays and thus the non-upgraded Silver packets can catch up with the upgraded ones to force less reordering. 
Finally, we show in Figure 81 and Figure 82 the Silver and Bronze throughput and goodput of both the DS and SLA models to highlight the advantage of the SLA model over the DS model. Clearly, for both the Silver and Bronze traffic, the SLA model achieved much higher goodputs although the policed rates at the ingress are the same for both models. The SLA achieves this advantage by upgrading the lower class traffic to use the upper class gaps. As can be seen from the figures, there exists a discrepancy between the Throughput and the goodput. This discrepancy is due to network losses as investigated in the next section.

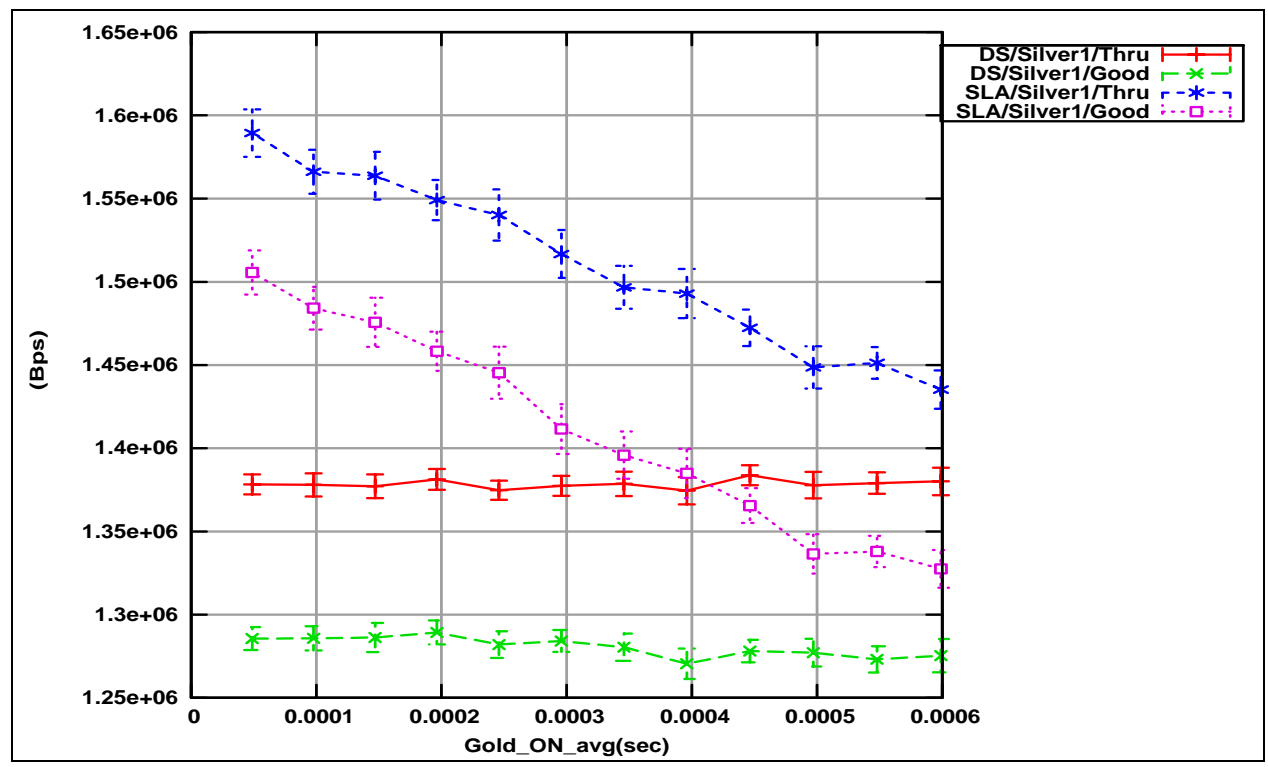

Figure 81 Client1 Silver Throughput and Goodput Versus Gold's ON period $\left(1 / \mu_{1}\right)$ 


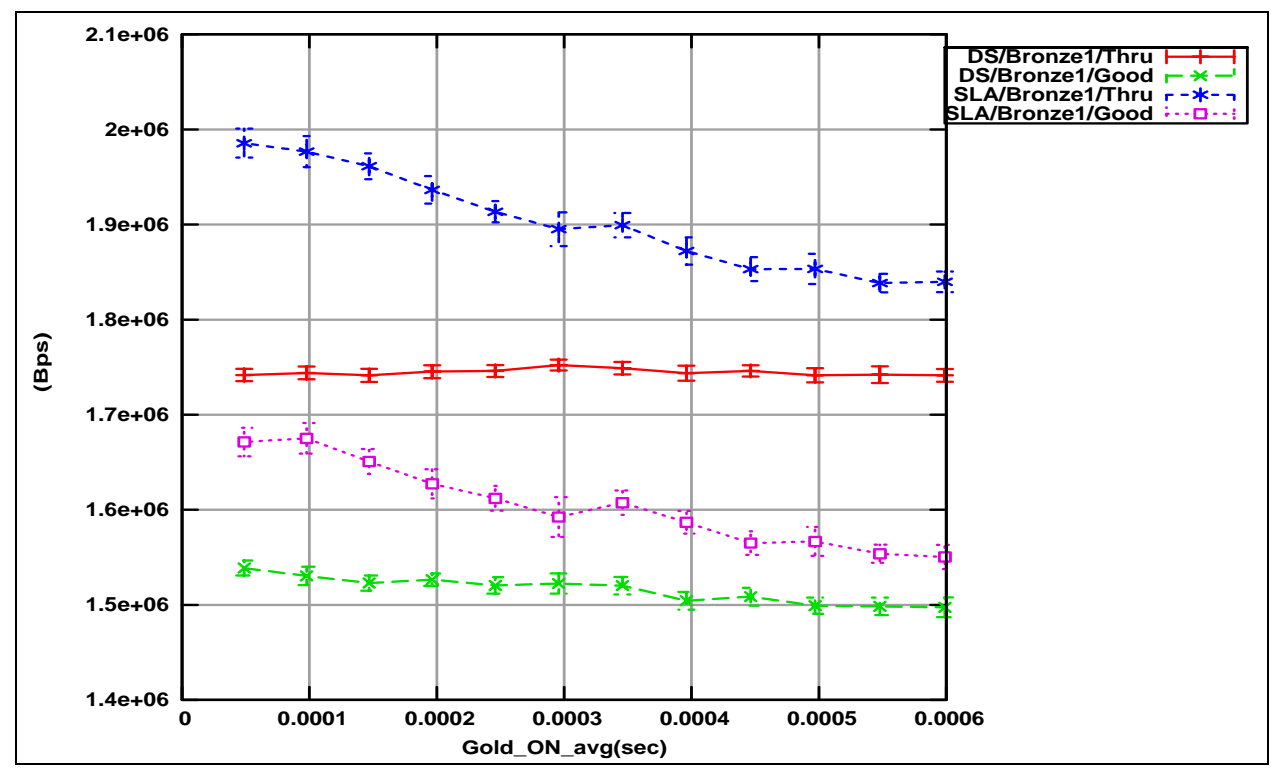

Figure 82 Client1 Bronze Throughput and Goodput Versus Gold's ON period $\left(1 / \mu_{1}\right)$

\subsubsection{Packet loss}

Losses can occur due to ingress policing or due the RED buffer management algorithm inside the network. The ingress policing is based on a leaky bucket algorithm configured with a CBS, CIR pair of parameters. Since our sources are bursty, due to IPP arrivals, we expect some drops due to long bursts when the rates are conformant to CIR.

We will start by showing Client1's ingress drop rates, due to the ingress policing for the various models, as the Gold rate is increased. Figure 83 shows that the Gold ingress drop rates are almost the same for the 3 models, since all models drop the excess Gold rate (as the Gold rate goes higher, bursts are more likely to be trimmed by the leaky bucket policer). Figure 84 shows that the DS Silver ingress drop rate is independent of the Gold rate, but the SLAR and the SLA models Silver ingress drop rates increase as the Gold rate is increased due to smaller gaps in the Gold bandwidth, and thus less upgrades. Finally, Figure 85 shows the Bronze ingress drop rates, and again, as the Gold rate is increased, the less the Gold gap 
which forces the Silver packets to use the Silver bandwidth instead of being upgraded, which in turn tightens the Bronze gap causing excess Bronze traffic to be dropped. However, from the referenced figures, it is clear that the upgrade model (both SLAR and SLA) allows more client traffic into the network, whereas the DS model blocks excess traffic from using higher class gaps.

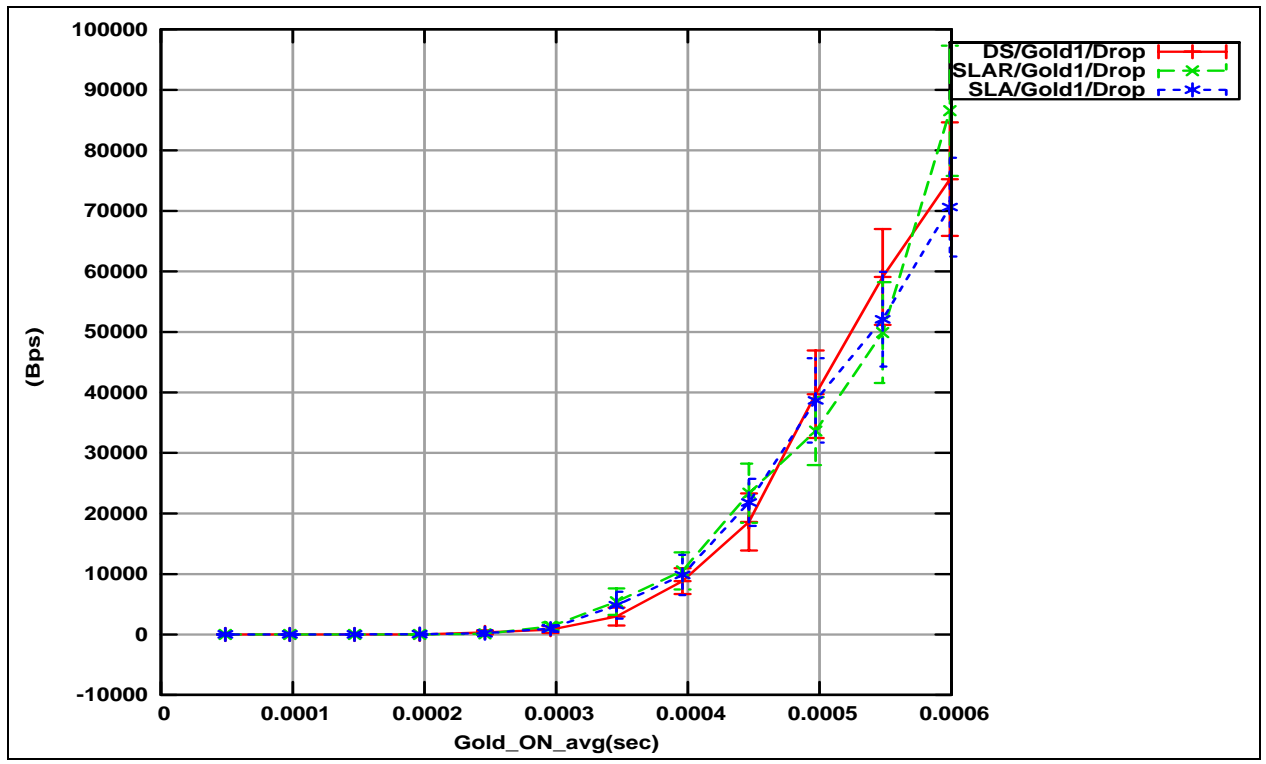

Figure 83 Client1 Gold Drop Versus Gold's ON period $\left(1 / \mu_{1}\right)$ 


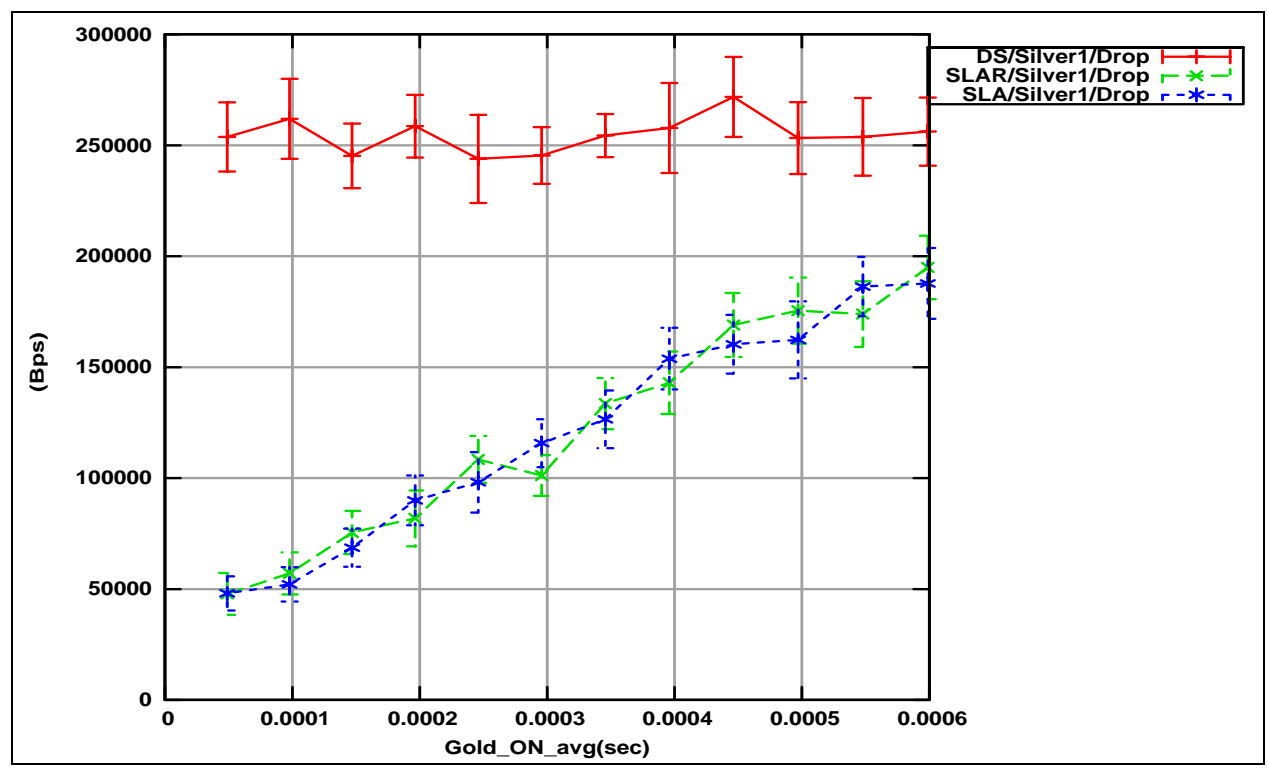

Figure 84 Client1 Silver Drop Versus Gold's ON period $\left(1 / \mu_{1}\right)$

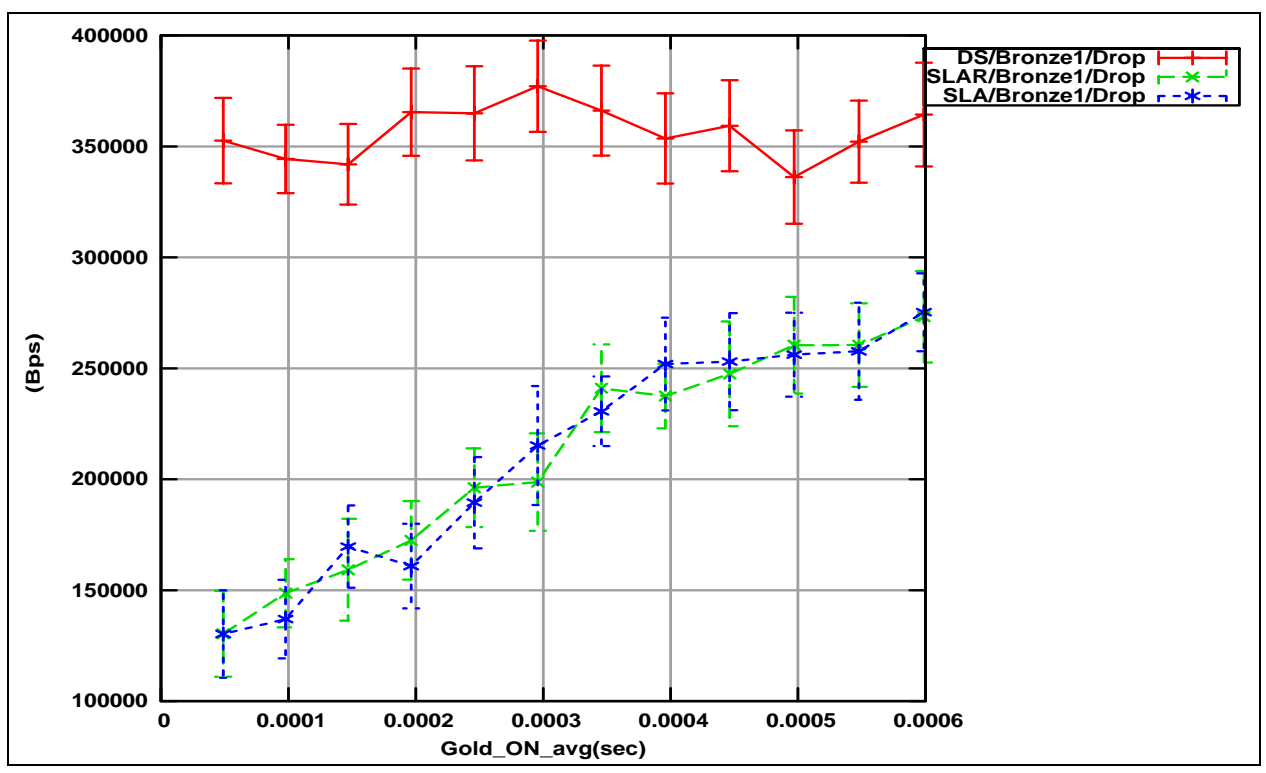

Figure 85 Client1 Bronze Drop Versus Gold's ON period $\left(1 / \mu_{1}\right)$

After looking at the drop rates due to ingress policing, let us investigate the total number of packets lost due to both ingress policing and the RED buffer management (note here that packets that were reordered in the network will be considered lost and thus accounted for in the total losses). Figure 86 shows Client1's total number of Gold packets 
lost. As can be observed from the figure, all models have similar losses for the "best" traffic class. However, when investigating the total number of Silver and Bronze packets lost, as shown in Figure 87 and Figure 88 respectively, we observe that the SLAR model experienced heavy losses (due to reordering), and the SLA model experienced the least losses. The SLA model clearly outperforms the other 2 models due to upgrades and no reordering in the network.

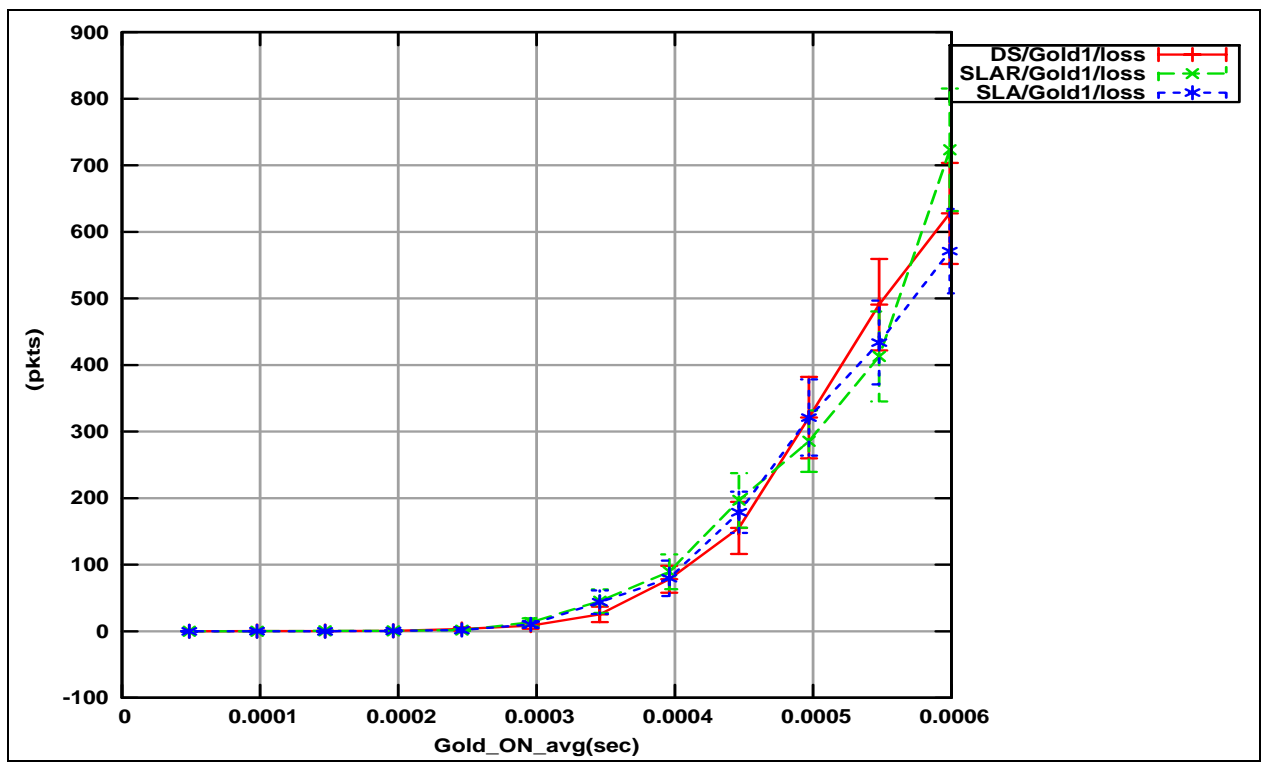

Figure 86 Client1 Gold Losses Versus Gold's ON period $\left(1 / \mu_{1}\right)$ 


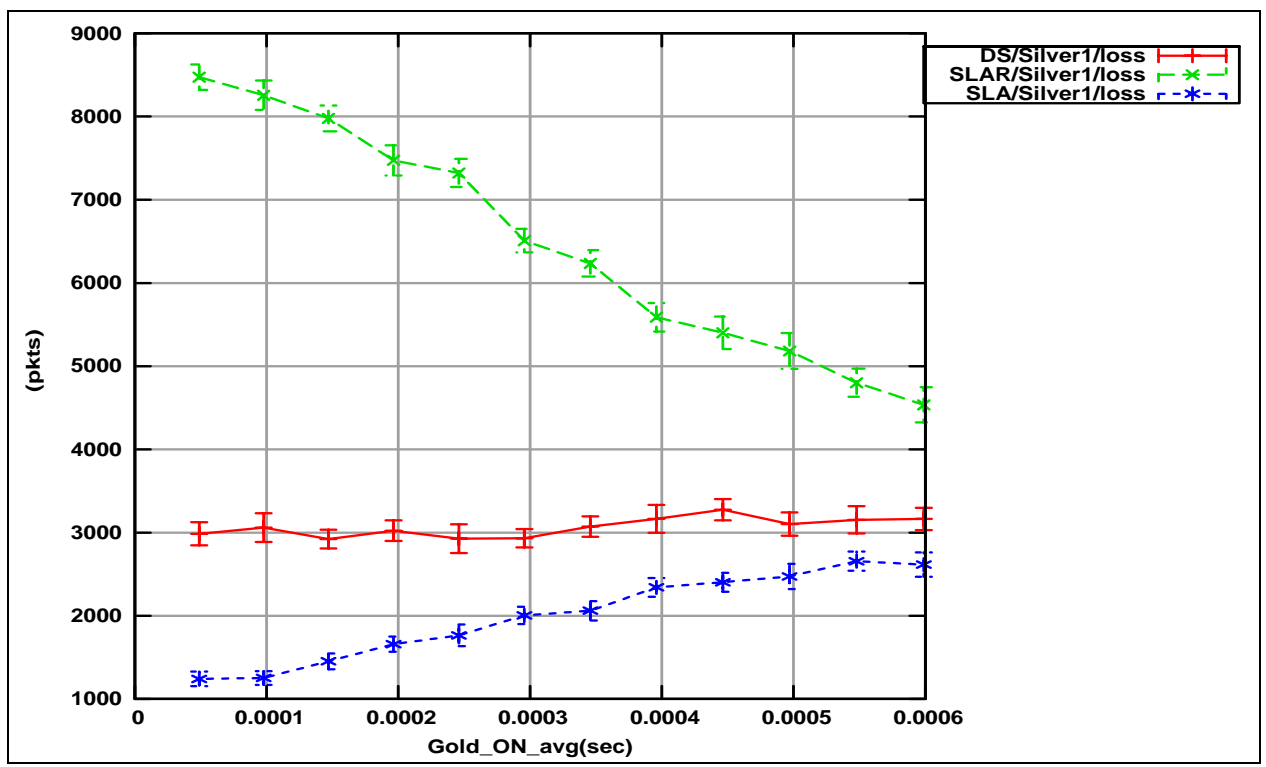

Figure 87 Client1 Silver Losses Versus Gold's ON period $\left(1 / \mu_{1}\right)$

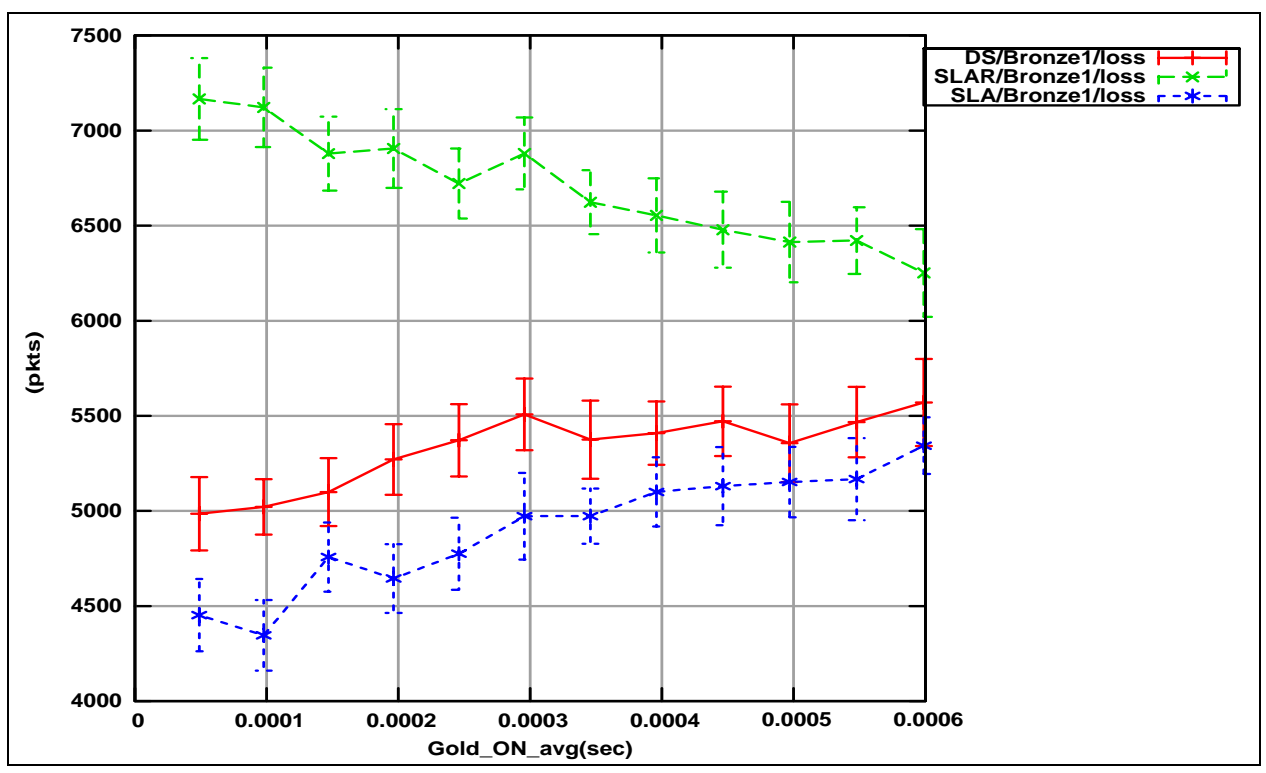

Figure 88 Client1 Bronze Losses Versus Gold's ON period $\left(1 / \mu_{1}\right)$

\subsubsection{End-to-End Delay}

We have seen previously that the SLA model outperforms the other 2 models in terms of goodput and losses. We will see in this section that upgrading packets to fill up the higher class bandwidth does add some queuing delay when compared to the DS model, however, we 
will argue that the added delay should fall well within the maximum acceptable delay for that class.

Let's look first at Client1's Gold delay versus the Gold rate as shown in Figure 89. The Gold rate is increased by increasing the ON period, which is a sequence of back to back (or burst of) packets that will have to be policed on ingress and then queued in each hop of the network. As the Gold rate increases, the burst size increases. This explains why the Gold packets experienced higher delays with increasing Gold rate. Notice that the delay is almost the same for all 3 models, with the SLAR and the SLA being slightly higher within the same Gold rate. This is due to the extra upgraded Silver packets that will be logically queued as Gold packets (tokens) and thus take Gold bandwidth. However, the SLAR or the SLA model does not add to the maximum Gold delay which is measured at maximum Gold rate (full CIR). Notice that the maximum Gold rate is the same for all models, and thus, if the maximum Gold delay for the DS model is acceptable for the customer for the Gold class, it should also be acceptable for all packets even if the maximum rate is not fully used. From this reasoning we can conclude that, since the SLAR and the SLA models will never allow rates to go beyond the configured or policed rates for a certain class, the SLAR and the SLA maximum delay can not exceed the DS maximum delay corresponding to the maximum DS policed rates. This reasoning is not class-specific, and thus applies to all classes. 


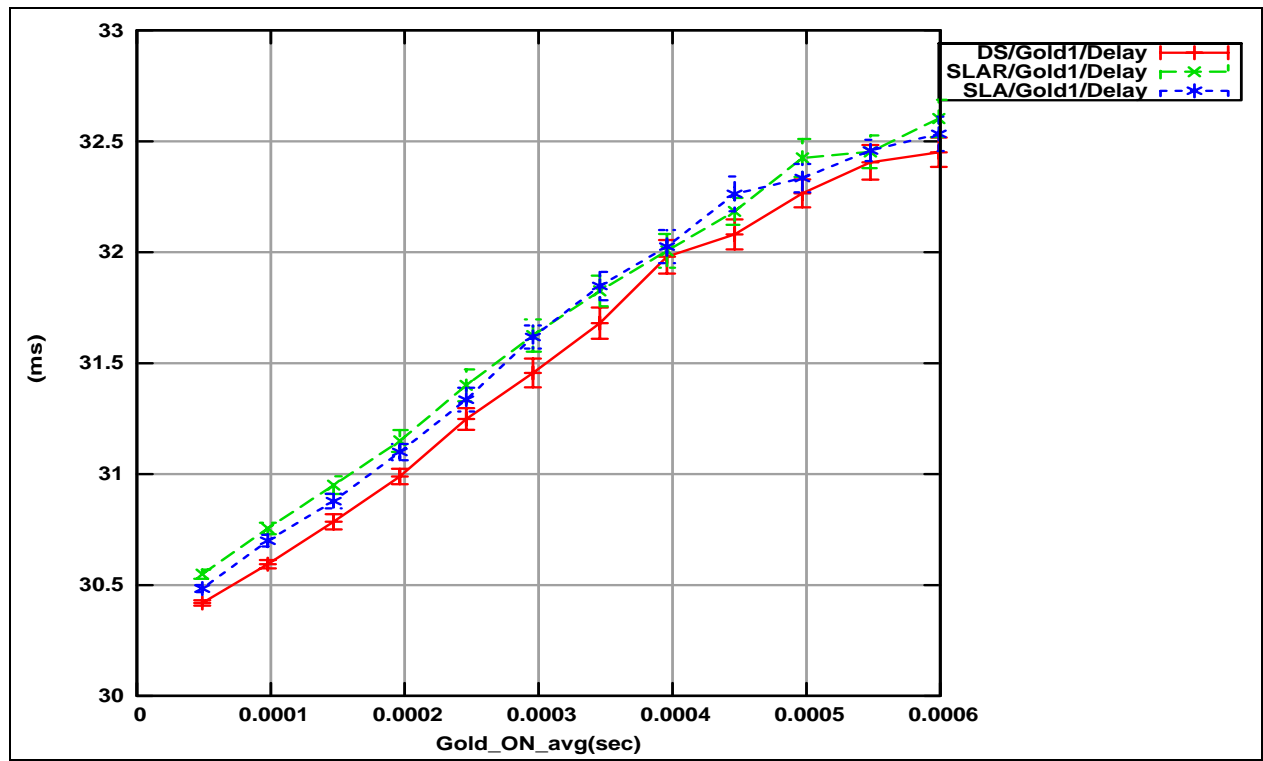

Figure 89 Client1 Gold Delay Versus Gold's ON period $\left(1 / \mu_{1}\right)$

Figure 90 and Figure 91 compare Client1s Silver and Bronze delays for the 3 models.

Notice that all 3 models tend to converge, as the Gold rate increases, towards the maximum rate delay (as argued above). The SLAR model "appears" to have a lower Silver delay than the other 2 models since the delay was computed using the non-reordered received packets (reordered packets were assumed lost and not accounted for in the delay computation). Also, the received SLAR upgraded packets took the faster Gold path in the network and thus further contributed to reducing the delay. 


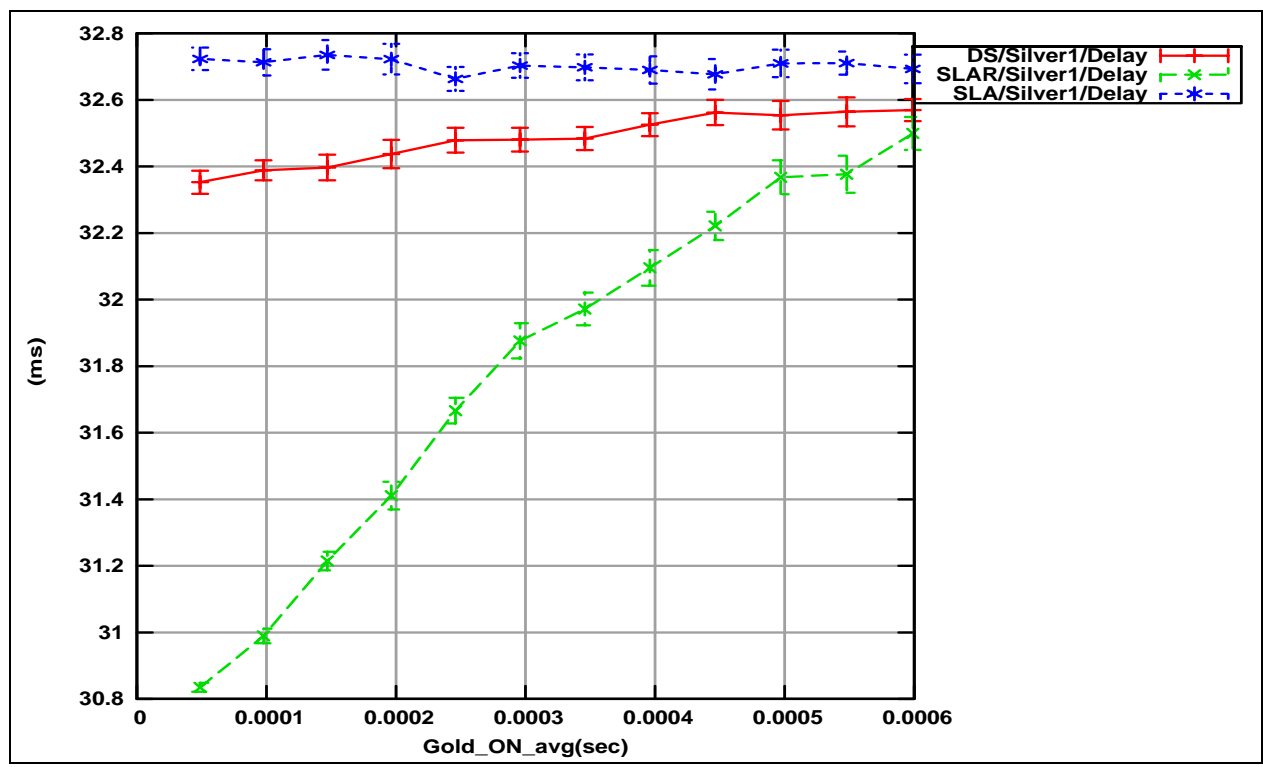

Figure 90 Client1 Silver Delay Versus Gold's ON period $\left(1 / \mu_{1}\right)$

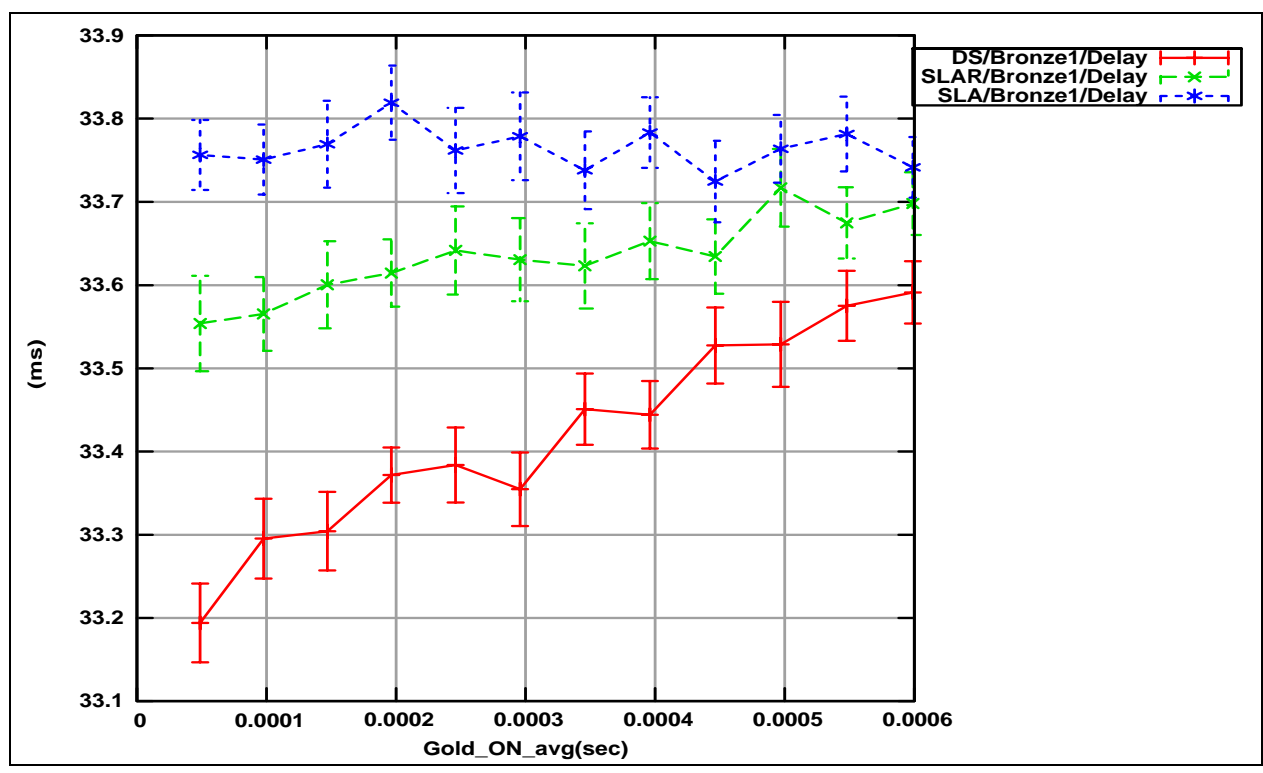

Figure 91 Client1 Bronze Delay Versus Gold's ON period $\left(1 / \mu_{1}\right)$

Finally, we show in Figure 92 the various classes' delays for the SLA model when varying the Gold rate. Since we are using WRR scheduling in the network, the Gold rate does not affect the Silver and Bronze servicing and thus does not affect their end-to-end delay. 


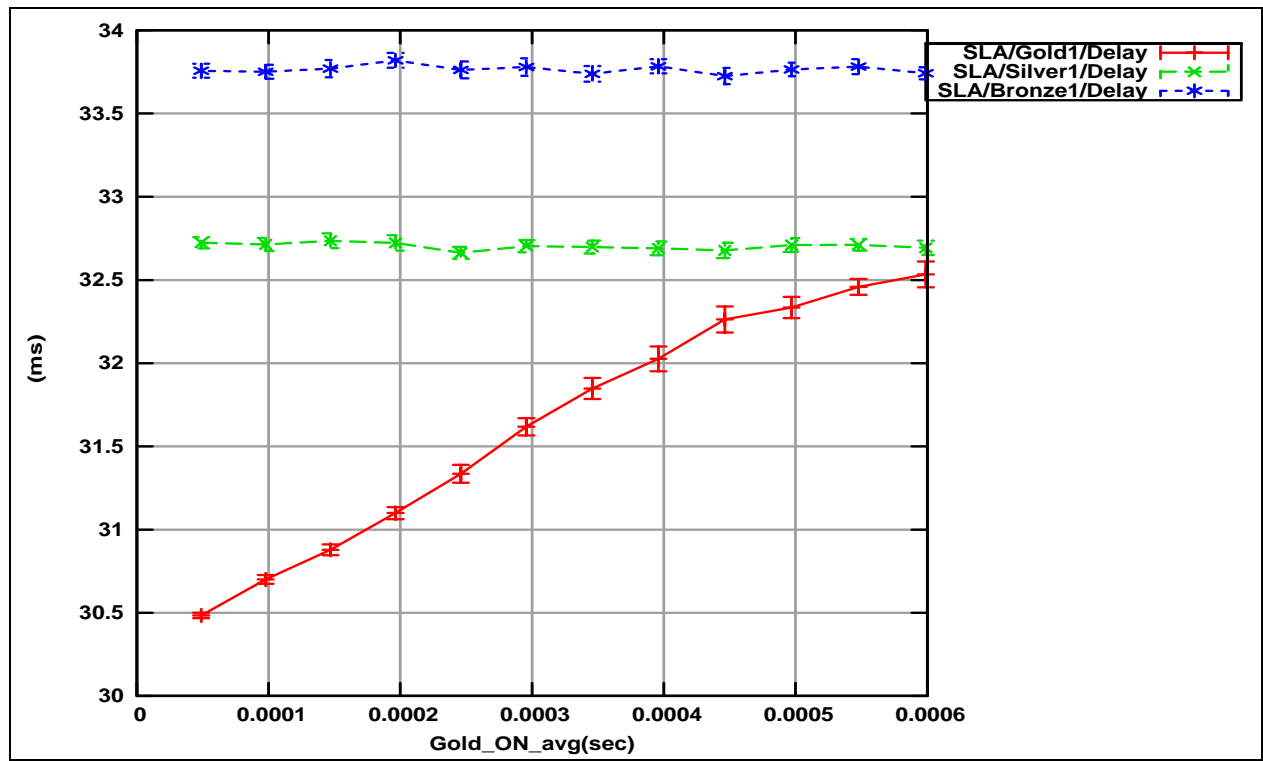

Figure 92 Client1 SLA Delays Versus Gold's ON period $\left(1 / \mu_{1}\right)$

\subsection{Summary and Observations}

In this chapter, we analyzed the behavior of the SLA under bursty traffic over higherspeed links, emulating provider-provider boundary, with lower propagation delay, emulating US East-to-West coast propagation delay.

We used IPP arrivals with high coefficient of variation to generate bursty traffic and showed that the SLA behavior did not depend on the traffic type and that the SLA model outperforms the DS and SLAR models.

We demonstrated that the benefit of the SLA is that lower classes use the gaps in higher classes, which is paid-for but unused bandwidth, to get the needed "extra" bandwidth. The benefit is directly proportional to the gap size; the bigger the unused bandwidth in the higher classes, the bigger the advantage of the SLA over the DS model for example. In particular, over a provider-provider boundary, it is expected that the negotiated rates should be relatively much higher than on a customer-provider boundary. For that, we have shown 
that over OC3 links speeds (considered relatively low provider-provider link speeds) the "client" provider can get a fairly better service when compared to the DS model with no upgrades. 


\section{Chapter 8}

\section{Experimental Results - TCP Highlights}

In Chapter 6 and Chapter 7 we analyzed the behavior of the SLA for UDP traffic, comparing it with the behavior of the DS and SLAR models. In this chapter, we focus on evaluating the TCP behavior, highlighting some TCP specific issues.

We first present in section 8.1 some TCP background information, such as algorithms used for congestion avoidance and the impact of packet reordering. Then in section 8.2 we describe the simulation configuration used in this chapter. In Section 8.3, we display our first experiment using a tight ingress policing scenario to highlight several TCP related issues. In section 8.4 , we repeat the experiment with a looser policing on ingress to allow for full TCP rates. In section 8.5, we create a gap in the Gold class and show how the Silver TCP could use this gap to get better rates, however bounded by the TCP maximum rate. In section 8.6 , we increase the RED thresholds in order to investigate whether the thresholds (or the average queue sizes) aggravate reordering. In section 8.7, we show the effect of the ingress policer's initial bucket size. In section 8.8 , we highlight the importance of the upgrade model when the RTT can not be estimated a priori to SLA setup. In section 8.9, we investigate whether the 
TCP flavor (Reno, Tahoe and SACK) affects the SLA behavior. Finally, in section 8.10, we summarize our findings in this chapter.

\subsection{TCP background}

TCP is a reliable, connection oriented, transport layer protocol designed to reliably transfer data from one end of the network to the other. It entails mechanisms to recover from data that is lost, duplicated, or delivered out of order by the internet communication system. This is achieved by assigning a sequence number to each octet transmitted, and requiring a positive acknowledgment (ACK) from the receiving TCP. If the ACK is not received within a timeout interval, the data is retransmitted. At the receiver, the sequence numbers are used to correctly order segments that may be received out of order and to eliminate duplicates.

TCP provides a means for the receiver to govern the amount of data sent by the sender. This is achieved by returning a "window" with every ACK indicating a range of acceptable sequence numbers beyond the last segment successfully received. The window indicates an allowed number of octets that the sender may transmit before receiving further permission.

TCP uses two main flow control mechanisms during data transfer: the receiver's flow control and the sender's flow control. The receiver's flow control is based on the receiver's advertised window size; with each ACK it sends to the sender, the receiver includes its available buffer size. A variable called the congestion window (cwnd) defines the maximum number of segments the sender can send without getting an ACK. Several algorithms control the size of the congestion window, two of them (slow start and congestion avoidance) come into effect during every data transfer. Two others, fast retransmit and fast recovery, come 
into effect during packet dropping or packet reordering. All four algorithms are defined in [88]. Here's a brief description of each of those algorithms:

- Slow start defines the way in which cwnd is initially set and accumulates its value. Initially, when a TCP connection is established, the congestion window is set to a default value ( 2 segments). The sender increases its congestion window by one segment with each ACK. At some point a limit is reached: cwnd may grow larger than the receiver's advertised window, or a limit along the path may be reached beyond which the network cannot send the amount of data being transferred (packet losses might occur due to congestion). At this point, the congestion avoidance algorithm takes control of cwnd.

- The transition to congestion avoidance algorithm is controlled by a variable called slow start threshold (ssthl). When cwnd is less than or equal to ssthl, slow start is in effect; otherwise congestion avoidance is in effect. Under congestion avoidance, the cwnd growth slows down to a rate of $1 /$ cwnd per each arriving ACK resulting in a linear growth rate of one segment for each RTT. Once congestion is encountered, as indicated by a dropped packet or timeout, the value of ssthl is set to half the current window, while cwnd is set to one causing the sender to go back to slow start.

- The fast retransmit algorithm is a method to detect a packet loss without waiting for a timer expiry. Once the sender detects three duplicate ACKs, it will deduce that a packet is lost and consequently a retransmit of the missing packet is triggered.

- Once retransmit takes place, the sender enters fast recovery. Fast recovery differs in two ways from congestion avoidance during the period between the retransmit of the missing packet and the time a new segment is ACKed. First, instead of closing the 
window and setting cwnd to one, the window's size is set to ssthl plus the number of duplicate ACKs (ssthl is assumed halved in the same way as during congestion avoidance). Second, as the sender continues to receive duplicate ACKs, it opens the cwnd by one segment for each duplicate ACK. This behavior is continued until the retransmitted segment is ACKed where the sender exits fast recovery and the cwnd value is set to equal ssthl, congestion avoidance is initiated, and cwnd returns to linear growth.

As mentioned before, TCP uses two basic mechanisms to detect segment loss. First, if an ACK for a given segment is not received in a certain amount of time, the retransmission timer expires and the presumed lost data segment is retransmitted. Alternatively, TCP can use the fast retransmit algorithm which uses 3 duplicate ACKs to infer that a particular data segment was dropped by the network. Notice that a packet reordering of three or more positions within their flow will trigger fast retransmit and fast recovery, and actually affect the throughput.

Packet reordering not just causes the sender to drop its rate (since reordering could be detected as a lost packet), but also has other negative effects on TCP performance as shown in $[70,73,80]$. Here's a summary of how reordering could negatively affect TCP:

- As just mentioned, a network that reorders data segments such that 3 or more duplicate ACKs received at the receiver will be treated as a segment loss and the TCP throughput is reduced.

- Segment reordering causes interruptions to TCP's ACK clock thereby causing its transmission to be more bursty. TCP's standard congestion control algorithm does not allow TCP to send segments in response to duplicate ACKs (while the window size 
increases upon reception of each ACK, duplicate or not). So for example, if segments $\mathrm{S} 1, \mathrm{~S} 2$, and $\mathrm{S} 3$ are transmitted, and $\mathrm{S} 1$ is reordered to arrive later than $\mathrm{S} 2$ and $\mathrm{S} 3$, the receiver would receive ACK1, ACK1, and ACK4 respectively. Upon receiving ACK4 the transmitter now can send 3 more packets in a burst versus transmitting one new packet upon receiving each of ACK2, ACK3 and ACK4.

- Reordering of ACKs can also cause bursty TCP behavior.

- Segment reordering can prohibit TCP from sampling the Round-Trip-Time (RTT) as frequently as in an ordered stream. RTT is used to estimate the Retransmission Timeout (RTO) used by TCP to achieve reliable delivery.

Several TCP implementations are publicly found, each with enhancements on the congestion avoidance and flow control handling. From these, two well-known implementations are TCP Tahoe and TCP Reno [89]. The main difference between the two is that TCP Tahoe drops its window size to 1 upon detecting packet loss (timeout or 3 duplicate ACKs), whereas TCP Reno drops the window size to 1 upon timeout and to half its window size upon 3 duplicate ACKs. For a more detailed performance comparison of TCP Tahoe and TCP Reno refer to [90].

\subsection{Simulation Configuration}

In this scenario, we use the same topology of Figure 15 (Chapter 6 page 78); however, we focus on the analysis of the TCP performance (client2 or $\mathrm{n}_{-} \mathrm{s} 2$ in the figure). As mentioned in Chapter 6, client 2 assumes a 3-tier SLA with some guaranteed rates for the Gold, Silver and Bronze traffic classes from the network. 
The inner nodes have the same capacity of $10 \mathrm{MBps}$ and propagation delay of $10 \mathrm{~ms}$. The network parameters and values used are the same as the values used in Table 6 and Table 7 of section 6.2.2, except that we use a higher CIR $(2,634,308$ Bps instead of $1,834,308 \mathrm{Bps}$ ) for the Silver background traffic (client4). The reason why we use a higher Silver Background CIR will become apparent in the next section.

Note that, unless otherwise stated, we will use the TCP-Reno, as provided by the NS2 simulator, as our TCP sender. The TCP senders will assume an FTP application with infinite file sizes (the TCP sources will continuously have data to send to their respective receivers).

\subsection{Tight Ingress Policing with upgrades}

In this scenario, we study the effect of the background Silver rate on Client2's TCP performance. As the background Silver rate increases, we expect to congest the Silver pipe in the network. As the Silver pipe is congested, we expect the TCP Silver rate to react by decreasing its transmission rate due to losses and/or higher RTT due to higher queuing delays. In particular, for the DS case, since there are no upgrades on the network ingress, we expect a decreasing performance as the Silver pipe gets more and more congested. But how do the upgraded packets help towards improving the TCP throughput in both the SLAR and the SLA cases when compared to the DS model? We will attempt to answer this question shortly hereafter.

Figure 93 shows the DS, SLAR and SLA goodput at the TCP receiver as the Silver background traffic is increased from $\sim 180 \mathrm{KBps}$ to $\sim 2,200 \mathrm{KBps}$. Note that Table 6 on page 
81 shows that Client2's Gold, Silver, and Bronze classes each being policed at 153,600Bps with a CBS value of 5,000 Bytes.

We first note the DS TCP Silver behavior where the goodput is unaffected by the background traffic, but is well below the policed rate of $\sim 153 \mathrm{KBps}$. Then at CBR $8=\sim 1,700 \mathrm{KBps}$, it starts dropping even further.

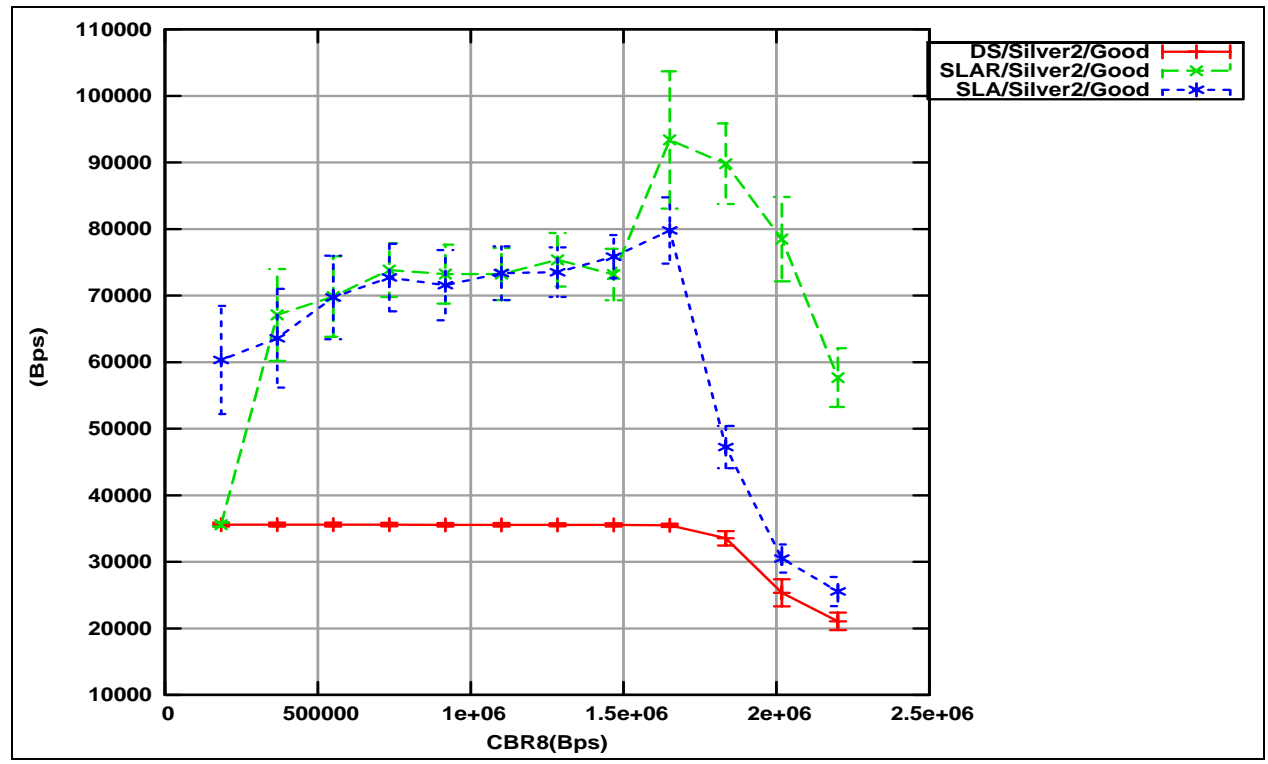

Figure 93 Silver TCP Goodput Versus Silver Background rate

Before we explain the DS Silver behavior, let's describe one of the TCP properties. As mentioned in section 8.1, the TCP throughput is in function of the congestion window (cwnd) and the Round Trip Time (RTT). One could approximate the maximum TCP rate as the maximum number of bytes sent per Round Trip Time (full TCP rate assuming a maximum window size of cwnd and an average RTT). In NS-2, the cwnd is in unit of packets and not bytes, so the maximum TCP rate is approximated as:

$$
\text { MAX_TCP_Rate }=\frac{\text { annd } * \text { Pkt_Size }}{\text { RIT }}
$$


where Pkt_Size is the average packet size used by TCP. Knowing the maximum configured cwnd, the average packet size, and the average RTT we can compute the approximated Maximum TCP rate; the NS-2 configured maximum cwnd was 20, the average packet size was 1,500 Bytes, and the RTT is a function of the network delay.

From the equation above, we can approximate the max_tcp_rate by knowing the average RTT. The average Round Trip time consists of the average forward (from sender to receiver) end-to-end delay plus the average reverse (from receiver to sender) path delay (assuming instantaneous packet ACK at the receiver). Figure 94 shows Client2's Silver average forward end-to-end delay. The figure shows that for low values of Silver Background rates, the DS average forward delay is about $61 \mathrm{~ms}$. As for the reverse path, since only TCP ACKs are flowing in the reverse direction, we assume the end-to-end delay in the reverse direction to be the sum of the propagation delays of the links, i.e. $60 \mathrm{~ms}$ (we ignore the queuing since the ACK rate is very small compared to the link capacities and we ignore the transmission times since ACKs are small 40-byte packets). So, the average RTT time is approximated at $121 \mathrm{~ms}$, which should yield a max_tcp_rate of $\sim 248 \mathrm{KBps}$ (compared to the observed 35KBps). This implies that Client2 Silver TCP must have observed heavy losses, even for small Silver Background traffic rates (i.e. even when there is no congestion in the Silver pipe). 


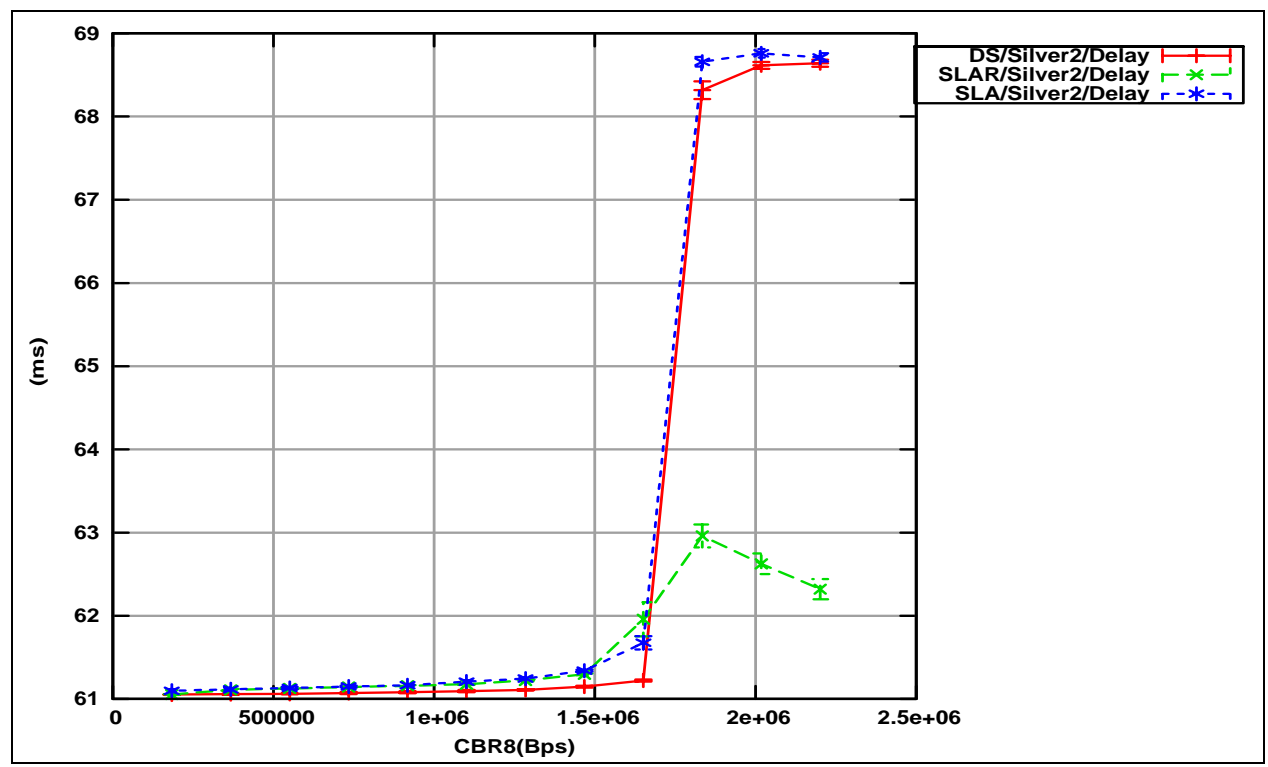

Figure 94 Silver TCP average forward delay Versus Silver Background rate

Remember that we are ingress policing at $\sim 153 \mathrm{KBps}$ with a CBS value of 5,000 Bytes. And remember also that a TCP packet loss could cause fast retransmit/recovery (halving the window size) in case of 3 duplicate ACKs or even slow start in case of a timeout (the fast retransmit/recovery depends on the TCP implementation; TCP Reno uses fast retransmit/recovery). Indeed, when we look at the ingress drop rates, as shown in Figure 95, we observe packet drops due to ingress policing $(3,000 \mathrm{Bps}=\sim 2$ packets per second), enough drops to cause TCP to adapt to a slower throughput.

One would expect that TCP would adapt to the policing rate (at $\sim 153 \mathrm{KBps}$ ), or something close to it. However, since the policer is a token bucket policer, the CBS parameter has a major impact on policing bursty sources. Since TCP relies on a cwnd window size to be transmitted, more or less in a burst, the CBS parameter needs to allow such a burst to pass. Notice that in our ingress policing we are using a CBS of 5,000 bytes but the NS-2 maximum TCP burst is $20 \times 1,500=30,000$ Bytes (cwnd x packet size). We will study the effect of CBS on TCP in more detail in section 8.7. For now, we know that we are 
tightly policing client2 TCP sources and, in order to allow for normal TCP flow control and congestion avoidance, we need to loosen the policing on ingress without messing up the provider CAC or reservation in the network.

Note that the Silver DS TCP flow starts dropping even further at CBR $8=\sim 1,700 \mathrm{KBps}$ (Figure 93). This is due to further drops inside the network (versus edge dropping) due to Silver pipe congestion as we increase the Silver background rate (notice also the average forward delay jump, Figure 94 , from $61 \mathrm{~ms}$ to $68 \mathrm{~ms}$ at $\mathrm{CBR} 8=\sim 1,700 \mathrm{KBps})$. The congestion is also indicated in Figure 95, where the congestion-losses cause TCP to drop its throughput well below the policed rate.

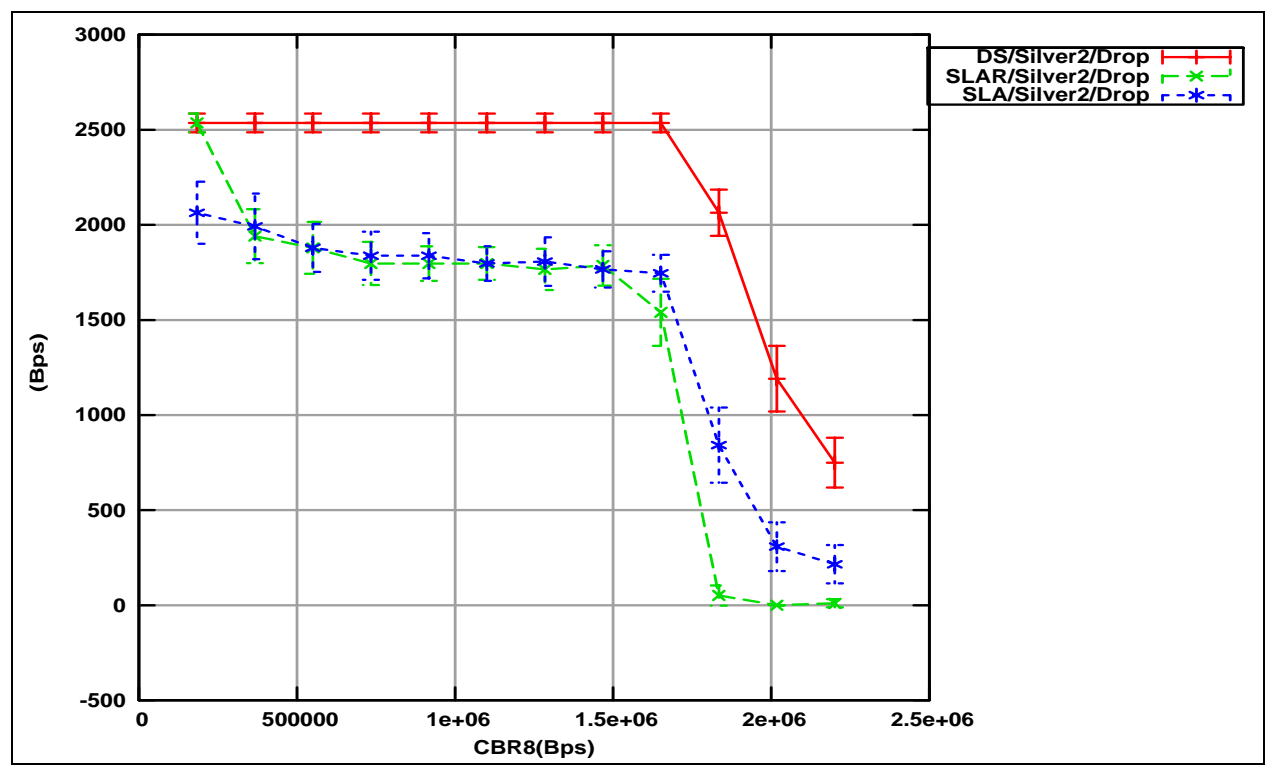

Figure 95 Silver TCP ingress drop rate Versus Silver Background rate

Now that we have described the DS behavior, we can analyze the SLAR and the SLA behavior. The SLAR and SLA models can use the upper Gold class to get extra bandwidth when available, and thus, due to upgrades, both the SLAR and the SLA models outperform the DS model. However, we know that the TCP rates are being tightly policed at ingress, and 
we also know that even if the Silver TCP can fully use the Gold rate (i.e. with full upgrades) it is still not enough to get near the maximum rate (due to CBS drops). But how does the SLAR model compare to the SLA model?

From Figure 93, we can observe that the SLAR, upon congestion, succeeded to have more throughput than the SLA model, a counter-intuitive result given the fact that the SLAR model may introduce reordering and that for TCP reordering, in some cases, means packet loss and thus rate drop and congestion avoidance. Two things to investigate here: first, why does the SLAR model out-perform the SLA model under congestion? And second, did we observe enough reordering to cause the SLAR model to under-perform?

To answer the first question, we need to consider differences in packet treatment between the two models. One main difference between the SLA and the SLAR, besides the reordering solution, is the fact that for SLAR, once a packet is upgraded to Gold for example, it flows in the network as a Gold packet until it reaches its destination. For the SLA case, the packet is inserted back into its original queue and a token is inserted in the upgraded class. Thus for the SLA, the packet flows in the network as its original class of service with some upgraded treatment once the token reaches the head of the upgraded queue. So, for the SLA, even though packets are upgraded, packets are still queued in the original class queue. So, if the background Silver traffic is congesting the Silver pipe, Silver queues along the pipe will be large. Moreover, since we are using RED for buffer management, if the average queue size reaches a certain minimum RED threshold, a new packet arrival will get dropped with a certain probability (the probability is $100 \%$ when the average queue size reaches the maximum RED threshold). Note that the Gold queue is not monitored by RED since we 
expect that the Gold queue sizes in the network to have small sizes (Gold service is targeted for VoIP like services which require little to no delay).

To recapitulate, queuing packets in the Silver queue versus the Gold queue, exposes the packet to a certain drop probability depending on the average queue size (RED). When the average queue size is larger than the RED minimum threshold, the probability of dropping is bigger than zero. Thus, in a network where the Silver service is congested, we expect losses. For TCP, packet losses mean drop the transmission rate and retransmit the lost packets.

On the other hand, since for the SLAR upgraded packets travel on the Gold pipe, the SLAR can possibly get better service than the SLA model (upon Silver congestion). When the upgrade rate is high enough, the SLAR is expected to perform noticeably better than the SLA given that there are little to no packet reordering.

As for the question on whether reordering caused any performance degradation, remember that the TCP receiver does not discard out of order packets, and it does send an ACK with the last received in-order segment of data. So for example, assume that three segments are transmitted into the network, S1, S2 and S3, and that S2 and S3 are reordered. When segment S1 arrives, the receiver will transmit an ACK for S1. The next segment to arrive is $\mathrm{S} 3$, which is out of order. In this case the TCP receiver keeps $\mathrm{S} 3$ and ACKs the last in order segment received, S1 in our example. When Segment S2 arrives, the last in-order segment that has been received becomes S3 and the receiver ACKs S3. The reordering of S2 and S3 did not affect the transmitter throughput. Moreover, the receiver did not discard S3, so there was no packet loss. 
Although a single packet reorder does not affect the TCP throughput, a packet that is surpassed by three or more other packets will cause a TCP rate drop. For example, if packet S2 is surpassed by packets S3, S4 and S5 then the receiver will ACK the last in-order segment that has been received ( $\mathrm{S} 1$ in this case) for each of S3, S4 and S5. When the transmitter receives the third duplicate $\mathrm{ACK}$ for $\mathrm{S} 1$ it will be forced into the fast retransmit algorithm, thus halving the rate.

In general, assume a TCP receiver is expecting sequence number $\mathrm{SN}$, for reordering to cause a triple-duplicate ACK the following equation is satisfied:

$$
\left(\mathrm{SN}<\mathrm{SN}_{\mathrm{i}}\right) \text { and }\left(\mathrm{SN}<\mathrm{SN}_{\mathrm{i}+1}\right) \text { and }\left(\mathrm{SN}<\mathrm{SN}_{\mathrm{i}+2}\right)
$$

where $\mathrm{SNj}$ is the sequence number of the jth arrival.

In our simulations, we measured at the receiver the number of times the receiver transmitted 3 or more back-to-back duplicate ACKs as shown in Figure 96. Note that this measure for DS and SLA models indicates the number of packet losses detected by the receiver, whereas for the SLAR model it indicates both losses and reordering (since for DS and SLA, there is no reordering). Keep in mind that not all packet losses are detected by the TCP receiver. For example, if the last $n$ segments of an $m$ segment burst were lost $(n<m)$, the TCP receiver has no way to know that the transmitter has sent $\mathrm{m}$ segments. The TCP transmitter relies on timeouts to detect such losses. Note that the transmitter enters slow start when a loss due to timeout is detected. 


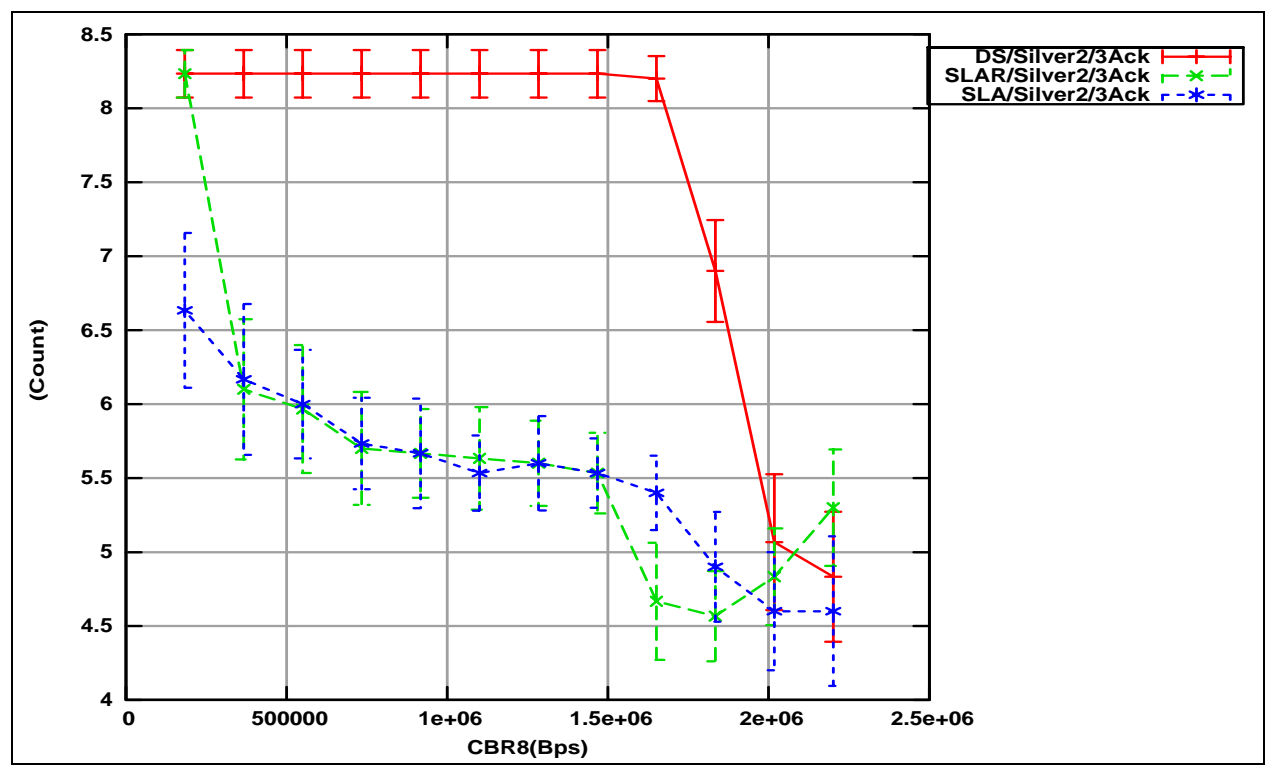

Figure 96 Silver TCP 3+ duplicate ACK count Versus Silver Background rate

Figure 96 shows that the DS model TCP receiver detected 8 losses within the time interval used to compute confidence intervals and sent on average around 8 times 3 or more duplicate ACKs back to the transmitter (we mentioned above that the DS heavy losses are due to ingress policing and no upgrades). As for SLAR case, as we reach the congestion period (i.e. $\mathrm{CBR} 8>1,700 \mathrm{KBps}$ ), the TCP receiver detected around 4 to 5 packets lost and/or reordered versus 4 to 5 packets lost for the SLA model. Referring back to Figure 94, we can see that the delay is much less for the SLAR case than the SLA case (due to packets flowing on the Gold pipe) which explains why the goodput in Figure 93 is better than the SLA case. In other words, for almost the same amount of 3 duplicate ACKs between the SLA and the SLAR model, the SLAR model achieves higher throughput since packets are flowing on the Gold pipe bypassing the Silver congestion. However, as indicated in Figure 96, the amount of reordering that causes the transmitter to enter fast retransmit is much less than expected. 
This is explained by the ingress policers' upgrade mechanism and the fact that TCP is a bursty traffic source; within a burst of $\mathrm{m}$ packets after a round trip period of no transmission (i.e. the buckets' tokens are replenished), the upgraded packets are most likely to be the first few packets within the burst consuming the upper class tokens and the tail few packets will be flowing as non-upgraded packets (since the previous packets within the burst should have consumed the upper class tokens). In this case, within a burst, since the first few packets get upgraded and the rest are not, the reordering probability is reduced (upper class pipes are considered faster). Reordering could happen though if within the next burst, the upgraded packets made it to the receiver before the tail packets of the previous burst. As an example, assume the Gold and Silver token sizes are 5000 bytes and that a burst of 6 TCP Silver segments (assume segment size is 1500 bytes) are being transmitted. In this case, the first 3 segments will be upgraded to Gold and would flow in the SLAR network as Gold packets, and the last 3 segments within the burst will flow as Silver packets in the network. After a round trip time when ACKs are received the window size is increased and another burst of packets is transmitted. For reordering to happen, the newly transmitted and upgraded packets must bypass the previous Silver packets. Keep in mind that at least 3 duplicate ACKs need to be generated by the receiver for the transmitter to drop its rate. As shown in the graphs above, for the network parameters chosen, this was a relatively low-frequency event.

To summarize this section's findings, we observed that:

- TCP maximum rate is approximated as cwnd/RTT.

- The CBS parameter of the token bucket policer impacts the TCP throughput (more details in section 8.7). 
- In order to allow for TCP flow control and congestion avoidance built-in mechanisms we need to loosen the tight ingress policing.

- $\quad$ The SLAR model has an advantage over the other 2 models upon lower class congestion; this is due to the fact that upgraded packets flow in the network on the upper class pipes bypassing the lower class congestion.

- The reordering characteristic of the SLAR model is dampened by the receiver buffering of out of order packets; the transmitter rate is affected only when 3 or more duplicate ACKs are received, a phenomenon that occurs when 3 reordered packets bypass a previous sequence packet (relatively rare).

- The mechanism to upgrade packets on ingress, favors upgrading the first few packets of a burst instead of the few tail packets; this property along with the bursty property of TCP (a burst every RTT time) helps dampening the effect of triple-ACKreordering.

\subsection{Loose Ingress Policing with upgrades}

In the previous section, we established that the low performance of TCP was due to the ingress edge policing. In this section, we will increase both the CBS and CIR policer parameters for Gold, Silver and Bronze classes to 32,000 bytes and 330,600 Bps, respectively, in order to compare the TCP performance of the DS, SLAR and SLA models (previous CBS was 5,000 bytes, and previous CIR was 153,600 Bps). Note that we previously approximated the maximum TCP rate to be around $(20 \mathrm{x} 1500) / 121 \mathrm{~ms}=250 \mathrm{KBps}$ for a max cwnd $=20$ packets, average packet size of 1500 bytes and an average round trip time of $121 \mathrm{~ms}$. 
Figure 97 shows the DS, SLAR and SLA Client2's Silver TCP goodput versus the background Silver rate. The figure shows that when there is no congestion in the Silver pipe (i.e. CBR $8<1,400 \mathrm{KBps}$ ), all three models have the same performance. As we mentioned earlier, all three models are bounded by the maximum TCP rate for a round trip time of $121 \mathrm{~ms}$ which was calculated to be $250 \mathrm{KBps}$, regardless of upgrades (the upgrades rates is shown in Figure 98). The forward delay is shown in Figure 99 (the backward delay is assumed to be $60 \mathrm{~ms})$. Notice in Figure 99 that the delay for the non-congestion zone is the same for all 3 models and is equal to $61 \mathrm{~ms}$.

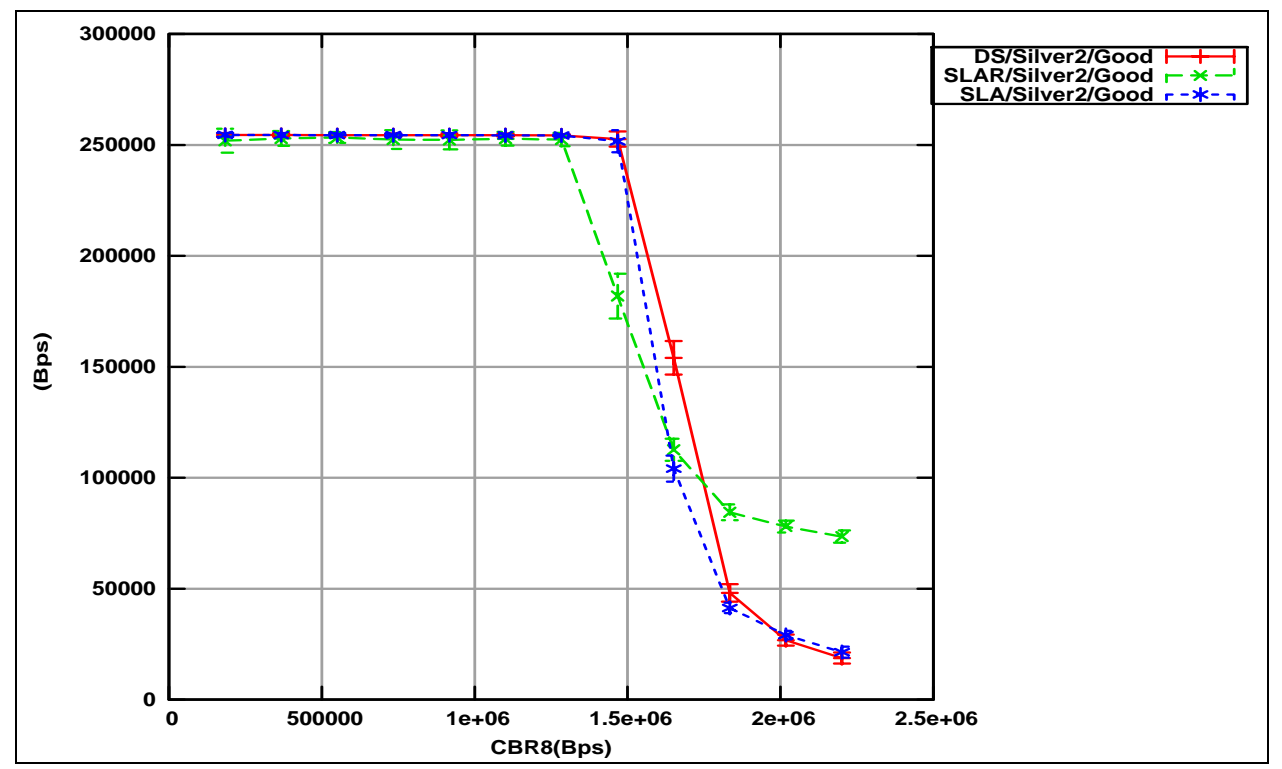

Figure 97 Silver TCP Goodput Versus Silver Background rate 


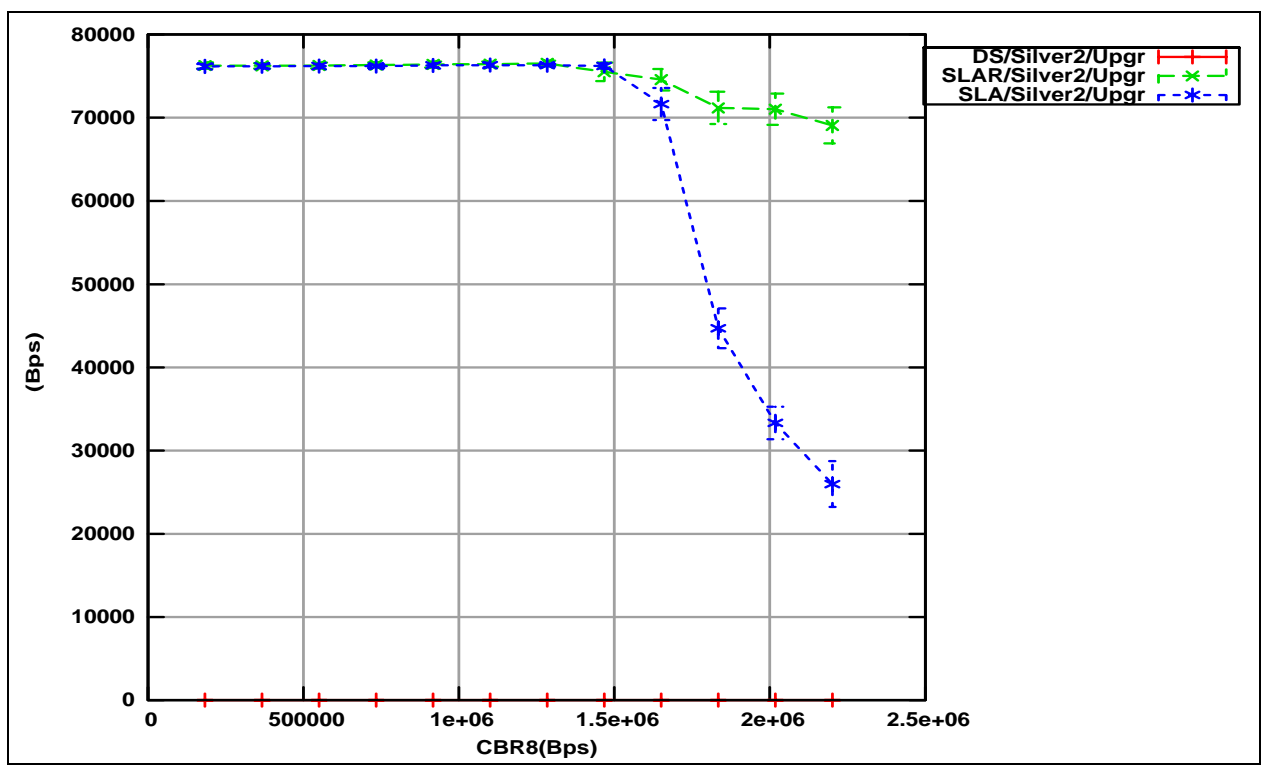

Figure 98 Silver TCP Upgrades Versus Silver Background rate

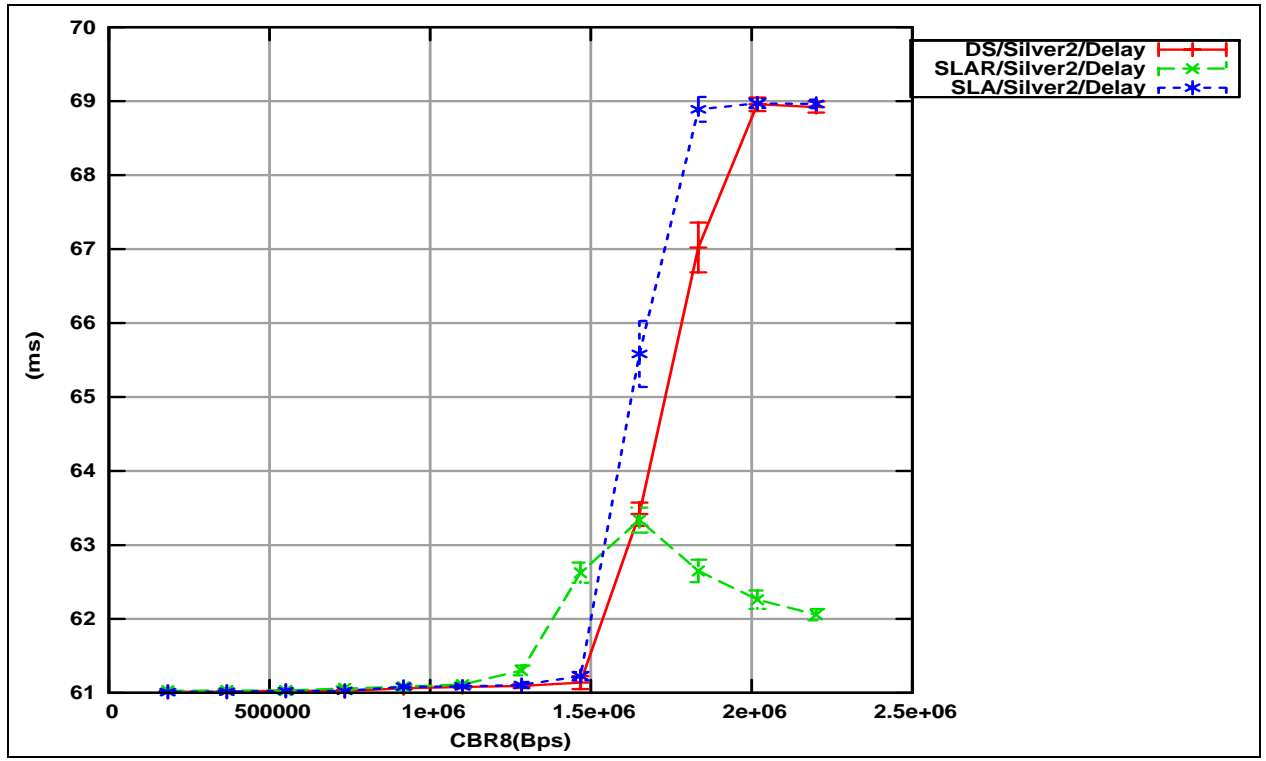

Figure 99 Silver TCP average forward delay Versus Silver Background rate

Notice in Figure 97 that the SLAR model has lower goodput at CBR8 $=1,450 \mathrm{KBps}$ than the other 2 models and then for CBR $8>1,750 \mathrm{KBps}$ the SLAR model gets a better goodput than the other 2 models. At CBR $8=1,450 \mathrm{KBps}$, both the DS and the SLA models did not observe any significant losses, so there is no reason why the SLAR model would 
observe losses, especially that the SLAR model uses the Gold pipe for upgrades bypassing Silver congestion. Looking at the 3 or more duplicate ACK count in Figure 100, we see that the SLAR model has a peculiarly higher count than the other 2 models. Remember that for the SLAR model, this count is both lost packets and reordered packets that could cause a transmitter rate reduction (fast retransmit). This is surely an indication of the latter (reordered packets) since neither the DS and the SLA models have observed losses at the same rate. This surely explains why the SLAR model performs poorly at CBR8 $=1,450 \mathrm{KBps}$ (a difference of $70 \mathrm{KBps}$ with the other 2 models).

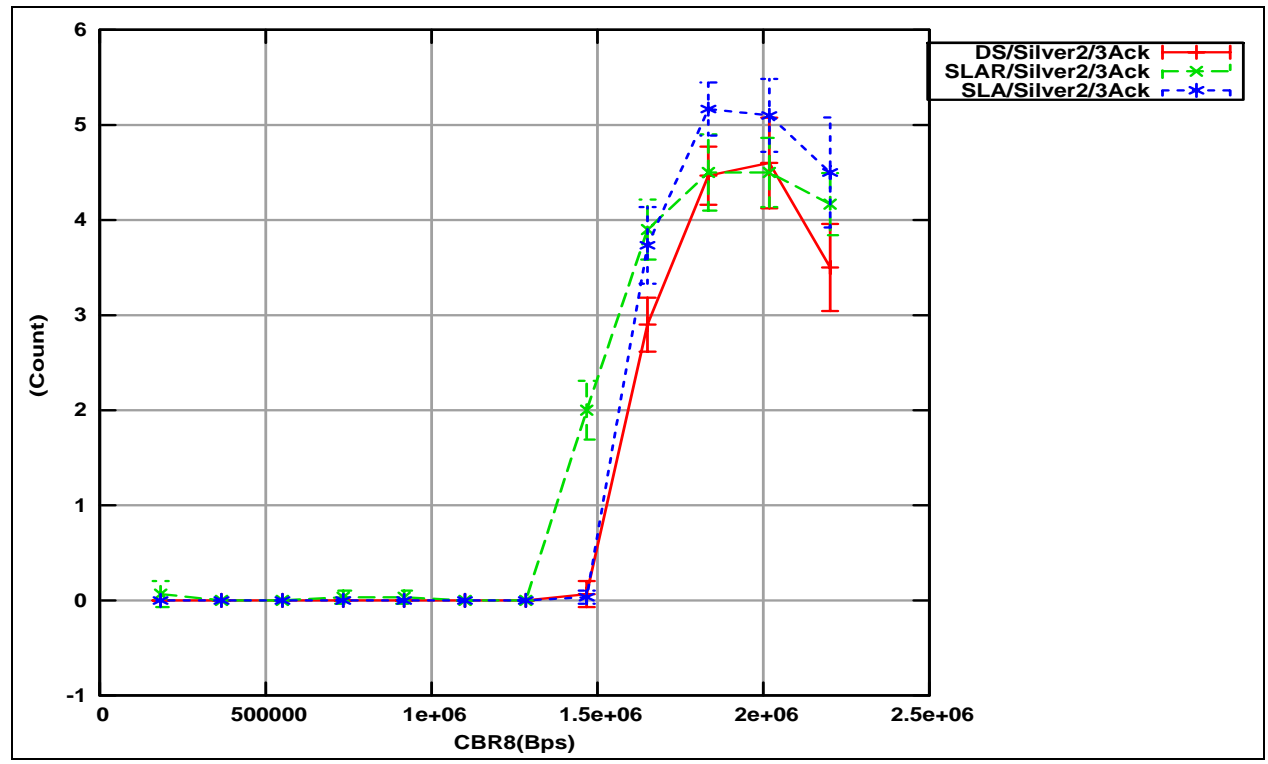

Figure 100 Silver TCP 3+ duplicate ACK count Versus Silver Background rate

The reason why the SLAR model outperforms both the DS and the SLA models during congestion periods (CBR8 $>1,750 \mathrm{KBps})$, is due to the fact that the SLAR model uses the Gold pipe to bypass the Silver congestion as explained in section 8.3. For relatively the same amount of 3 or more duplicate ACKs (as indicated in Figure 100) and relatively lower Round Trip Times (as indicated in Figure 99) the SLAR model has a better goodput. 
To summarize this section's findings, we showed that when we don't tightly police the TCP rates on ingress, all three models, DS, SLA and SLAR, have the same performance when there is little congestion in the network, even if we allow ingress upgrades. The reason for this was that the TCP rate is bounded by a maximum rate approximated by cwnd/RTT and would not take advantage of upper class upgrades beyond this limit. We also reinforced the findings of the previous section, in particular that the SLAR model has an advantage when a certain class (e.g. Silver) is congested, since upgraded packets flow on the upper class (e.g. Gold) bypassing the congested (i.e. Silver) class. Finally, we showed that the SLAR reordering characteristic affected the TCP throughput under certain conditions where the network is on the verge of congestion where the probability of upgraded packets reordering is maximized (Silver packets are delayed so that subsequent Gold packets bypass them). However, the TCP receiver dampened the reordering impact due to buffering out of order packets; only when reordering causes 3 duplicate ACKs that the TCP transmitter rate is affected.

\subsection{Using Gold gaps to upgrade the tightly policed Silver}

In the previous section we showed that TCP could not take advantage of upper class gaps, when loosely policed, due to the cwnd/RTT approximate maximum TCP rate. In this section, we slightly change the previous experiment by tightly policing the Silver and Bronze class while keeping a Gold gap in order to analyze the behavior of the three models and the advantage of upgrades. For that, we set the Gold policer parameters to [CBS $=32,000$, $\mathrm{CIR}=330,600]$, the Silver policer parameters to $[\mathrm{CBS}=32,000, \mathrm{CIR}=230,600]$, and the 
Bronze policer parameters to $[\mathrm{CBS}=32,000, \mathrm{CIR}=230,600]$ (all other parameters unchanged). Remember that the maximum TCP rate was approximated at 250KBps.

Figure 101 shows Client2's Silver TCP goodput versus the Silver background rate. The figure shows that the DS model was: a) tightly policed and was not allowed to use the gap in the Gold class, and b) that even though it was policed at 230KBps the goodput is around $150 \mathrm{KBps}$ for low congestion periods (i.e. CBR $8<1,500 \mathrm{KBps}$ ). The first observation is explained by the fact that the DS model does not automatically upgrade lower class packets to use bandwidth gaps in the upper classes. And the second observation is explained by the fact that packets dropped due to ingress policing force TCP to assume that the network is congested and cause it to drop its rate (either due to timeouts or due to 3 duplicate ACKs) below a certain point where the ingress policing allows packets to pass without drops.

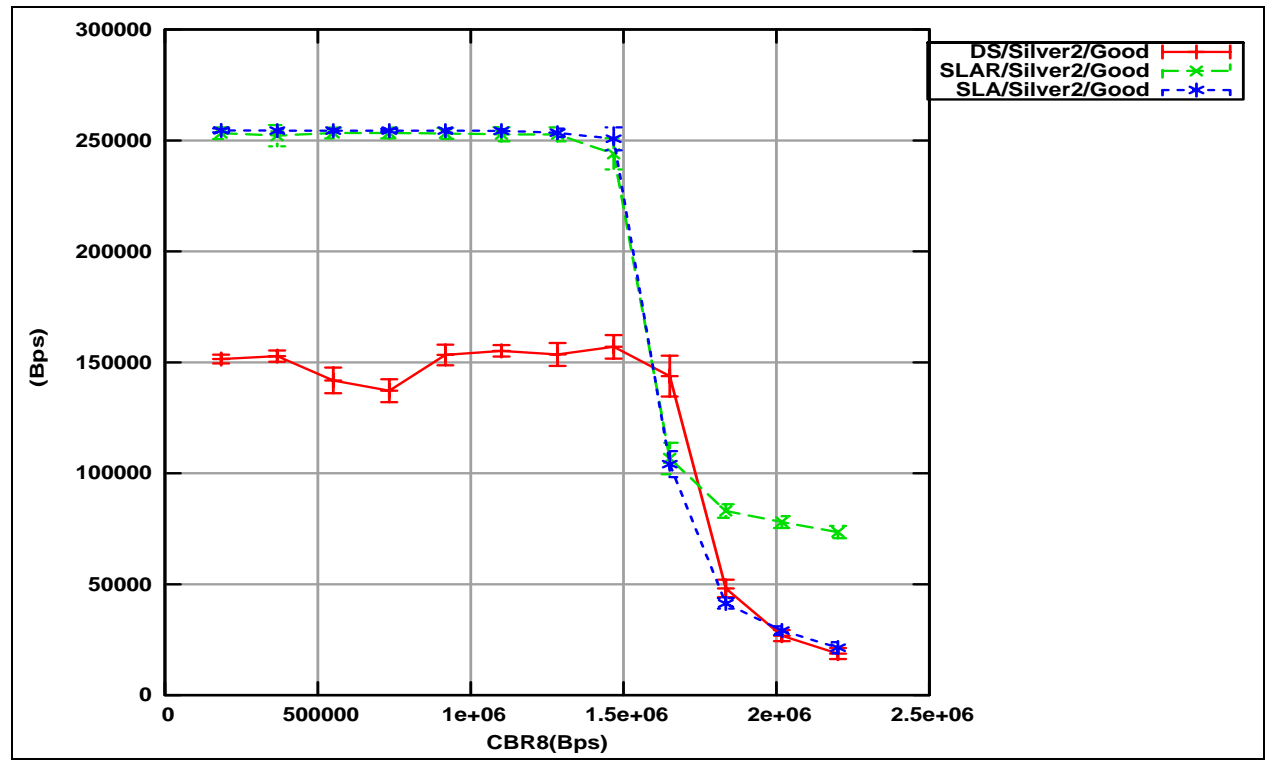

Figure 101 Silver TCP Goodput Versus Silver Background rate

Note on the other hand how the SLAR and the SLA models use the 330KBps $250 \mathrm{KBps}=80 \mathrm{KBps}$ Gold gap to get the full Silver rate (policed at 230KBps, the Silver TCP needs $20 \mathrm{KBps}$ to reach the approximated maximum rate of 250KBps). From Figure 102, we 
see that the SLAR model did not observe any reordering that caused 3 or more duplicate ACKs until CBR8 $=1,500 \mathrm{KBps}$, where it observed some (notice that at CBR8 $=1,500 \mathrm{KBps}$, there was no SLA model losses and thus the SLAR model 3 or more duplicate ACKs are due to reordering). As we mentioned earlier, reordering is aggravated when the Silver pipe is congested and the Gold pipe is not. However, when the Silver pipe starts observing some losses the loss effect starts affecting TCP rates. That is why for CBR $8>1,500 \mathrm{Bps}$, all 3 models goodput is negatively impacted. Finally, when the Silver pipe is almost clogged, the SLAR model uses the 80KBps Gold gap to get some goodput.

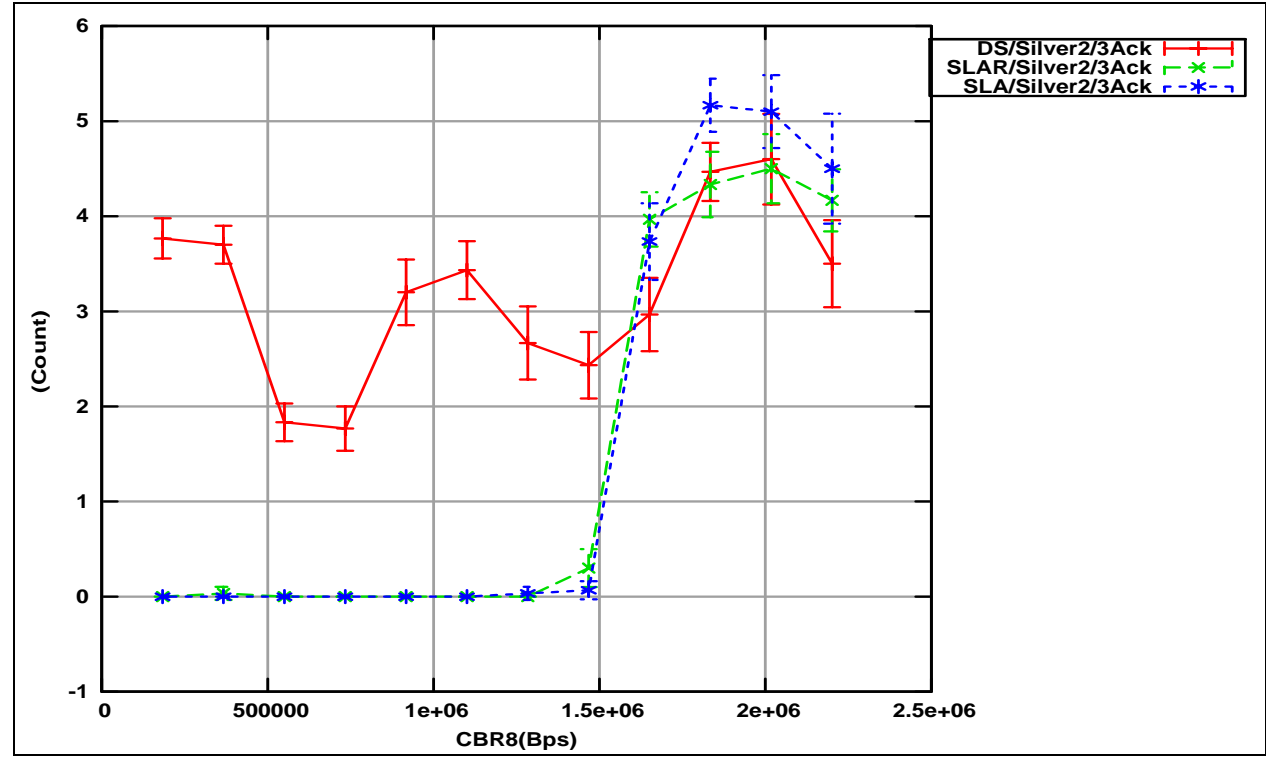

Figure 102 Silver TCP 3+ duplicate ACK count Versus Silver Background rate

Figure 103 shows the Silver TCP average forward delay versus the Silver Background rate. The DS model shows a slightly bigger forward delay than the other 2 models when there is no congestion, since the other 2 models are upgrading packets to use better service (using Gold time slots). For example, for a work-conserving scheduler, for the same arrival/service rates of Gold, Silver and Bronze queues, if we treat some of the Silver packets as Gold, the Silver packets should observe less average delay then if we don't treat 
some of the Silver packets as Gold (i.e. the Silver packets should observe higher service rates when upgraded). The SLAR model shows a lower forward delay during congestion since the upgraded packets are using the Gold pipe to bypass the congestion and the non-upgraded packets are mostly dropped due to congestion (the majority of the received packets are upgraded packets which contribute to a lower forward delay).

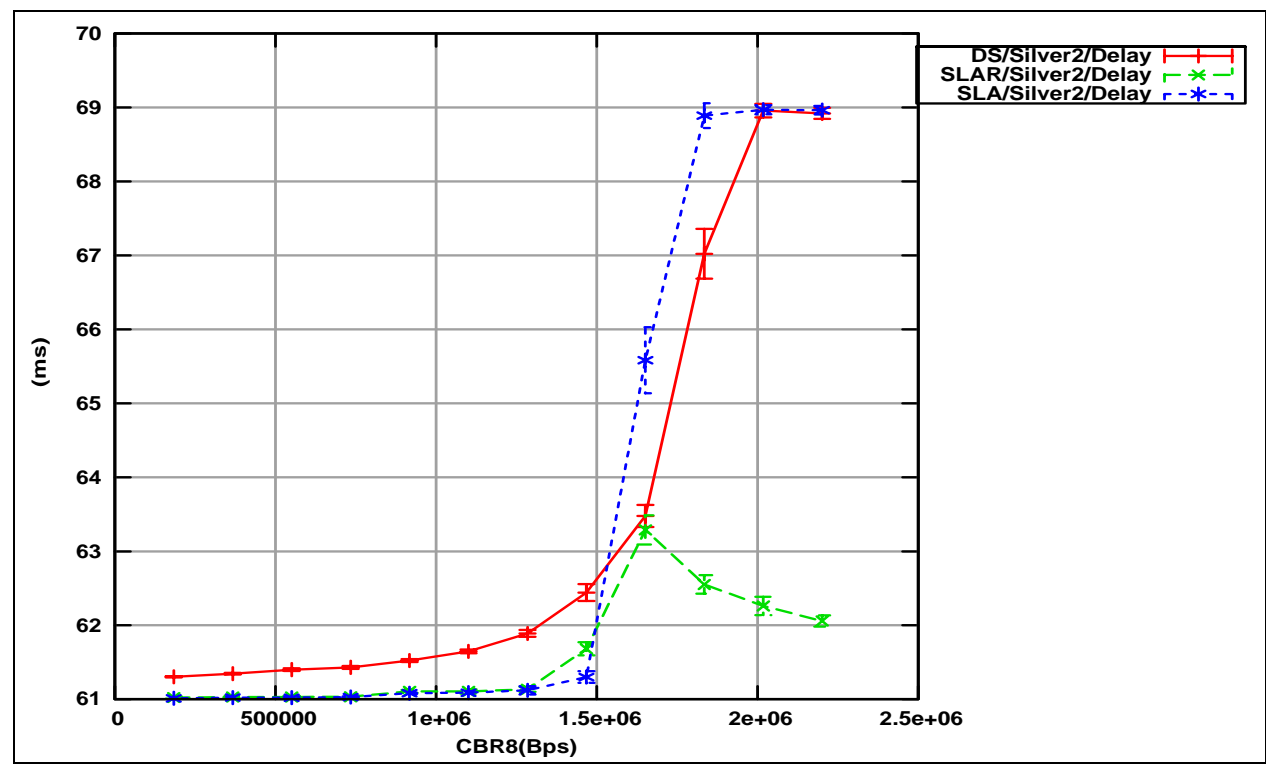

Figure 103 Silver TCP average forward delay Versus Silver Background rate

Figure 104 shows the Gold, Silver and Bronze SLA model TCP goodput. The figure shows that as the Silver background rate, the Bronze goodput is affected first due to Bronze congestion. In fact, when there is not enough Silver traffic in the network, the workconserving per hop WRR schedulers distribute the excess bandwidth to the other classes according to the assigned weights. So as the Silver rate is increased, the Bronze pipe looses the extra bandwidth to eventually converge to the guaranteed Bronze service rate as per the WRR scheduler; apparently, there is more Bronze traffic than the guaranteed Bronze rates in the network. Similarly, as the Silver background continues to increase, the Silver traffic saturates the guaranteed Silver service rates in the network, whereas the Gold rate is 
unaffected since the network total ingress Gold rate is less than the guaranteed Gold service rate (we mentioned before that the Gold service was designed for low queuing delay and thus the Gold ingress rate was chosen not to exceed the guaranteed Gold service rates).

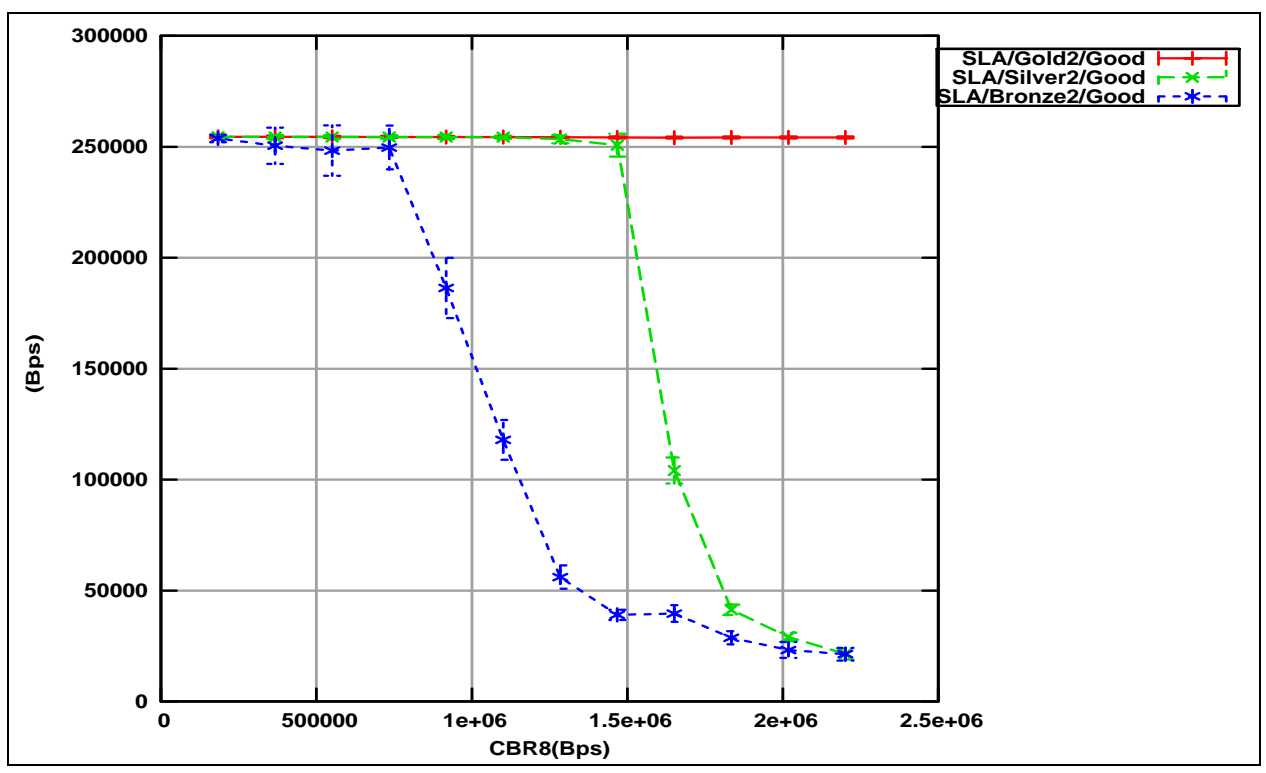

Figure 104 Gold, Silver and Bronze SLA TCP goodput Versus Silver Background rate

Figure 105 shows Client2's Bronze TCP goodput versus the Silver background rate. Again the figure shows that the SLAR and SLA models have the advantage of upgrades over the DS model when there is no congestion. However, the figure shows that there is a period of time just before we hit the Silver pipe congestion that the DS Bronze goodput was actually better than the other 2 models. This is explained by the fact that the SLAR and the SLA models upgrade Silver packets to use any Gold gaps. These accepted packets contribute to the total network ingress traffic rate. Thus, during the period of low congestion, for the same background Silver rates, the SLAR and SLA models have $80 \mathrm{KBps}$ more Silver traffic than the DS model. That is why the SLAR and the SLA models show Bronze congestion well before the DS model in Figure 105. As we keep on increasing the Silver background rate, the Bronze pipe starts converging to the guaranteed service Bronze rates. The SLAR and the 
SLA models have $80 \mathrm{KBps}$ extra Silver traffic and $80 \mathrm{KBps}$ extra Bronze traffic due to upgrades for $\mathrm{CBR} 8<750 \mathrm{KBps}$. For $\mathrm{CBR} 8=1,250 \mathrm{KBps}$, the SLAR and the SLA models have an extra $80 \mathrm{KBps}$ Silver and approximately $80 \mathrm{KBps}$ less Bronze than the DS model. Eventually, all three models converge when both the Silver and the Bronze pipes are completely congested (CBR8 $>1,750 \mathrm{KBps})$. Notice that the SLAR model does not get better service than the SLA model since the upgraded packets will be congested in the Silver pipe.

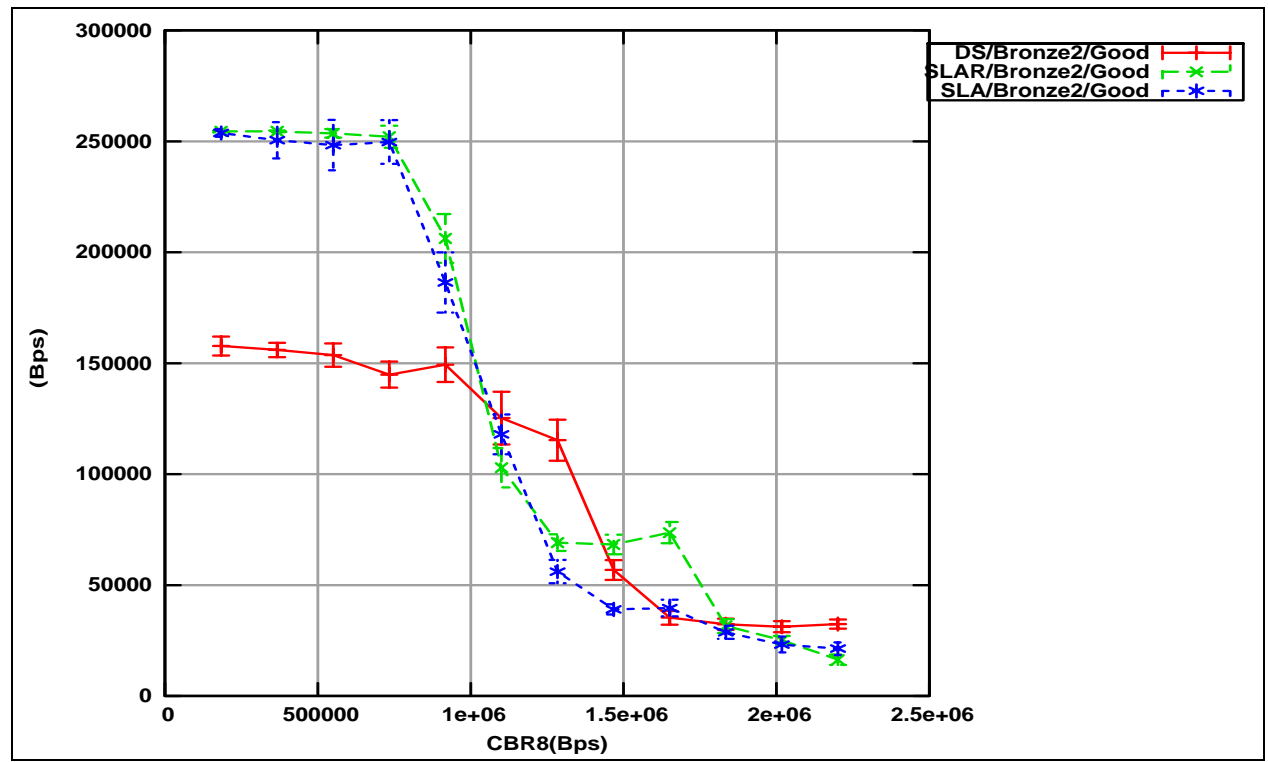

Figure 105 Bronze TCP Goodput Versus Silver Background rate

Figure 106 shows the number of 3 or more duplicate ACKs as the Silver background rate is increased. The figure confirms the congestion detection as the number of losses increase for both the SLA and the SLAR models. 


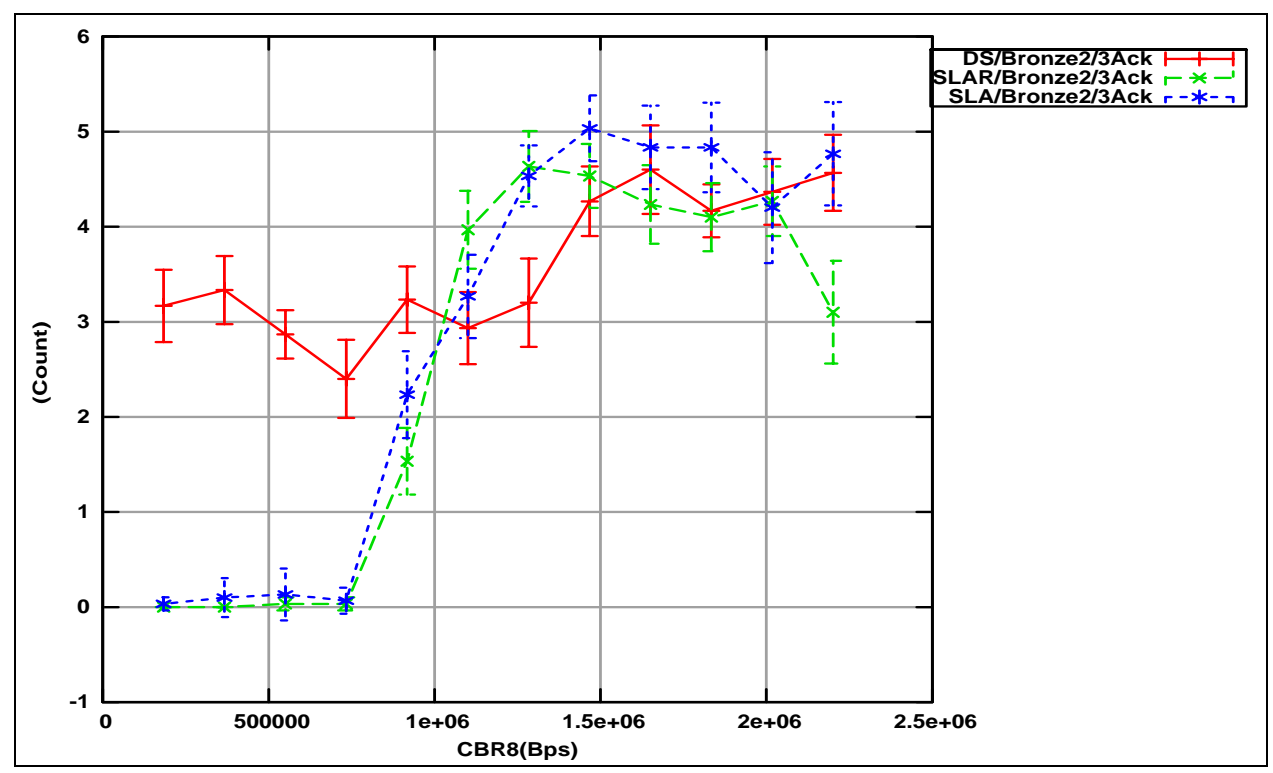

Figure 106 Bronze TCP 3+ duplicate ACK count Versus Silver Background rate

Finally, Figure 107 shows that as the Bronze pipe is congested, the SLA model observes the highest delays (again the SLA model's congestion occurs at a lower value of CBR8 due to upgraded Silver packets when compared to the DS model), since the SLAR model upgraded packets are using the still not congested Silver pipe. As the Silver pipe gets congested, all three models observe similar forward delays. 


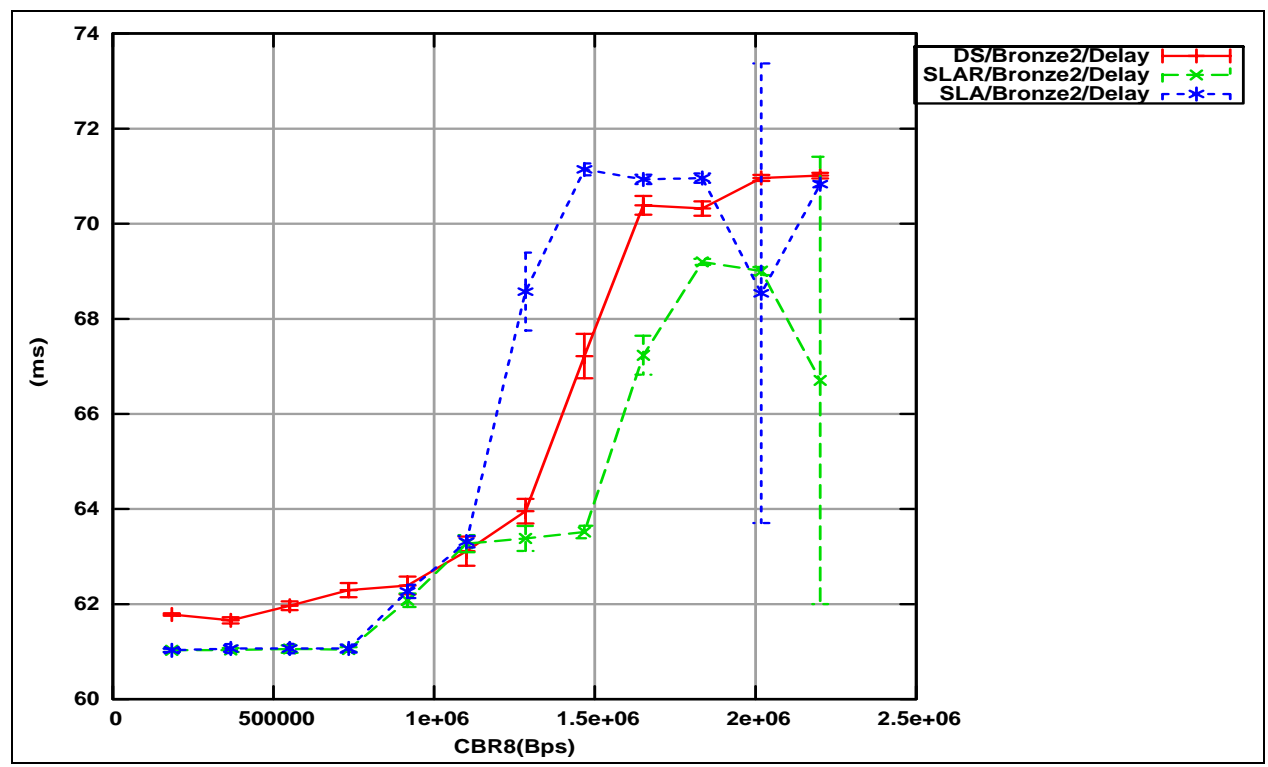

Figure 107 Bronze TCP average forward delay Versus Silver Background rate

To summarize this section's findings, we showed that lower-class TCP sources can benefit from gaps in upper classes when automatically upgrading lower-class packets to achieve higher rates; however, these rates are bounded by the maximum TCP rate approximated by cwnd/RTT. This means that, although upgrades are beneficial, TCP sources can not fully utilize the upper class gaps (since the TCP rate is bounded by the approximated maximum of cwnd/RTT) contrary to the UDP behavior shown in Chapter 6 and Chapter 7 . We also showed that the SLAR model's reordering characteristic is dampened by the TCP buffer at the receiver, and that it behaves better than the SLA model when there is congestion in the lower class but no congestion in the upper class.

\subsection{Effect of RED thresholds}

In the previous sections, we showed how the TCP Silver rate can benefit from upgrades, and argued that the SLAR reordering characteristic is dampened by the TCP receiver buffer. We also mentioned that the reordering is aggravated when the lower class 
pipe is almost saturated and the upper class is empty. But is this reordering of the type that causes TCP to drop its rate (i.e. is it "bad" reordering which causes 3 or more duplicate ACKs)?

In this section, we will vary the Silver RED thresholds along the Silver pipe in the network in order to cause larger Silver queue sizes (aggravate the reordering). Remember that the RED thresholds control the queue sizes, such that the lower the thresholds the lower the average queue sizes. We use the same parameters as in section 8.5 except that we set the Silver background rate value at 1,450 KBps which we observed earlier as a high enough value to cause "bad" reordering in the SLAR model without congesting the network.

Figure 108 shows Client2's Silver TCP goodput versus the RED min threshold (all the RED min and max thresholds in all nodes in the network were changed simultaneously). The maximum threshold, throughout this section, is equal to min threshold +5 (the drop probability between the two thresholds was set to 0.5 ).

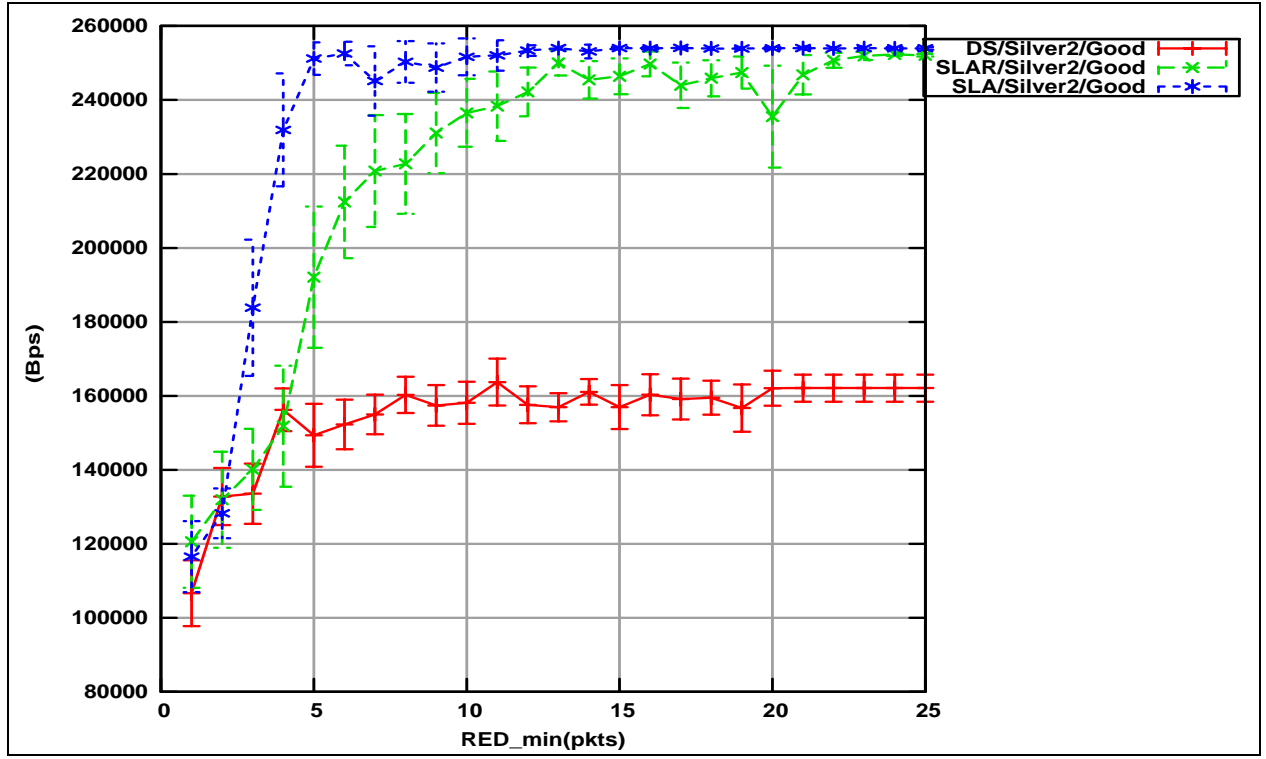

Figure 108 Silver TCP Goodput Versus RED min threshold 
The figure shows that as the RED thresholds increase, the DS goodput increases (up to RED_min=8) to stabilize for RED_min $>$ 8. When the RED thresholds are small, the Silver pipe average queue sizes are forced to be small by dropping packets, reducing the TCP thoughput. The DS throughput then stabilizes at $160 \mathrm{KBps}$ due to ingress policing rather than RED drops.

In order to understand the SLAR behavior, we need to look first at Figure 109 where we show the Background Silver traffic loss rate. The figure shows that the SLAR Silver background traffic has observed the most losses. This is due to the fact that in the SLAR model, upgrades flow on the upper pipe; so the Silver upgrades flow in the Gold pipe and the Bronze upgrades flow in the Silver pipe. Figure 110 shows the Silver and Bronze upgrades for both the SLAR and the SLA models. The figure shows that there was a lot more Bronze SLAR upgrades than the SLA case. The reason why we have more Bronze upgrades is that the amount of Bronze upgrades depends on the Silver rate measured at ingress. Since at low RED thresholds the Silver TCP backs-off due to network losses (due to low RED thresholds) as indicated by Figure 110, the Bronze rate gets more upgrades (the Bronze traffic observes less losses since the majority of the packets is not upgraded and thus flow in the Bronze pipe). As the thresholds are increased, the Silver TCP increases its rate and thus lowers the Bronze upgrade rate. When there is enough Silver buffer in the pipe, the Silver throughput reaches its maximum (notice from Figure 109 that the Silver pipe experiences losses up until RED_min $=23$ ). 


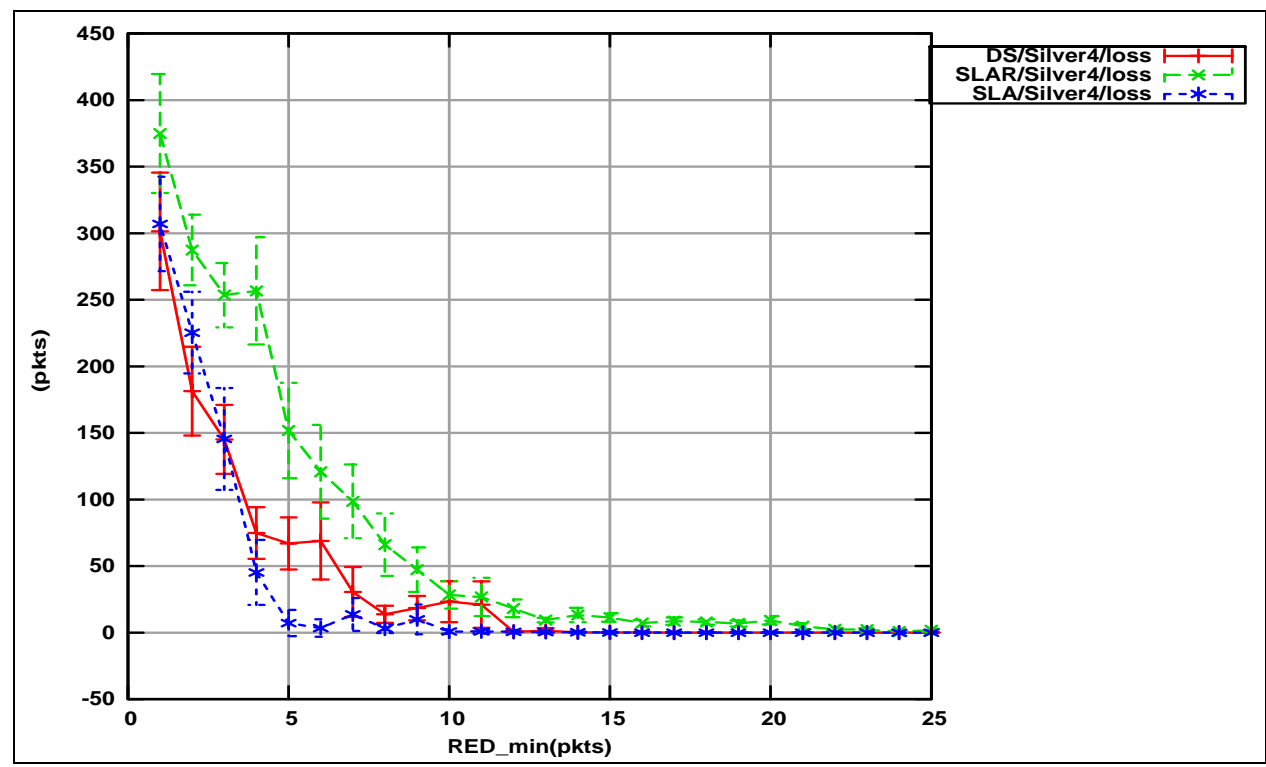

Figure 109 Silver Background loss rate Versus RED min threshold

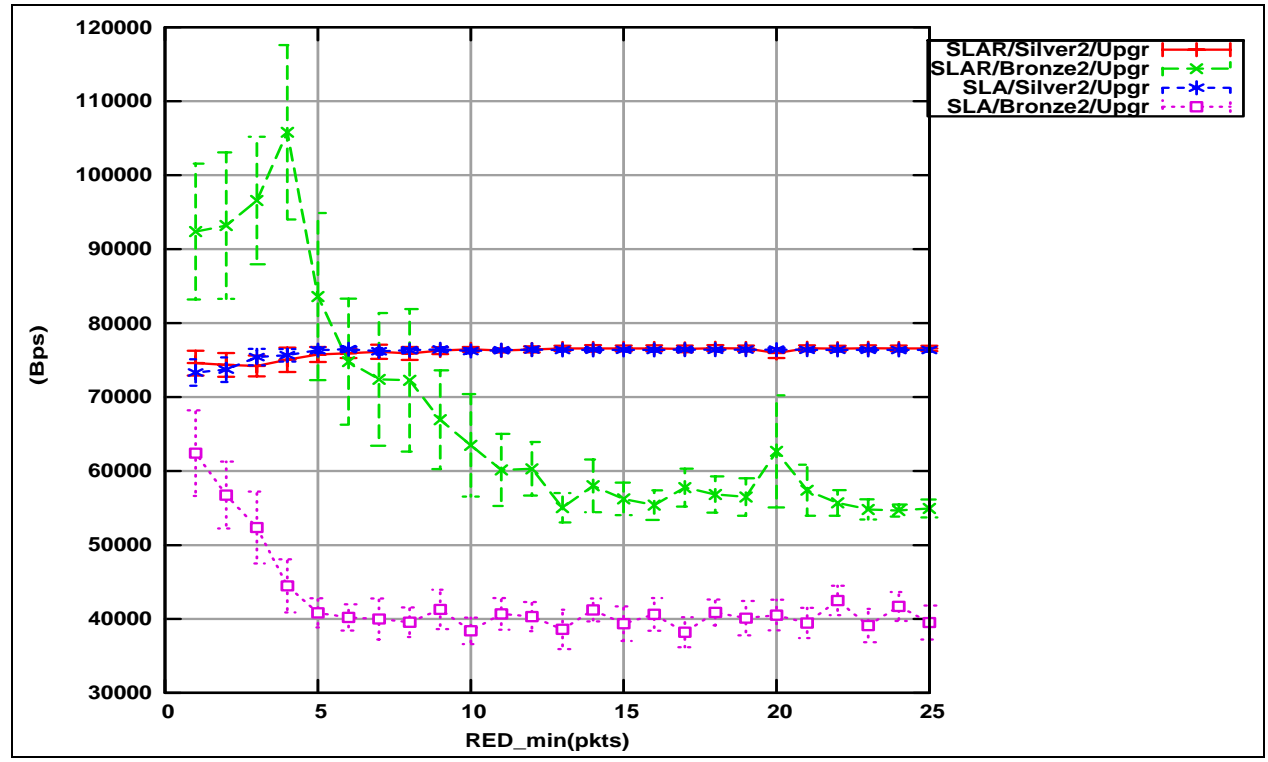

Figure 110 Silver and Bronze upgrade rates Versus RED min threshold

Plotting the number of times the sender sent back to the receiver 3 or more duplicate ACKs (shown in Figure 111), we can see that the SLAR model experienced more losses and/or reordering. Since we know for sure Silver losses occurred up until RED_min=23, it 
seems that reordering did happen but not as much as predicted (since the goodput in Figure 108 seems to be driven by losses more than "bad" reordering).

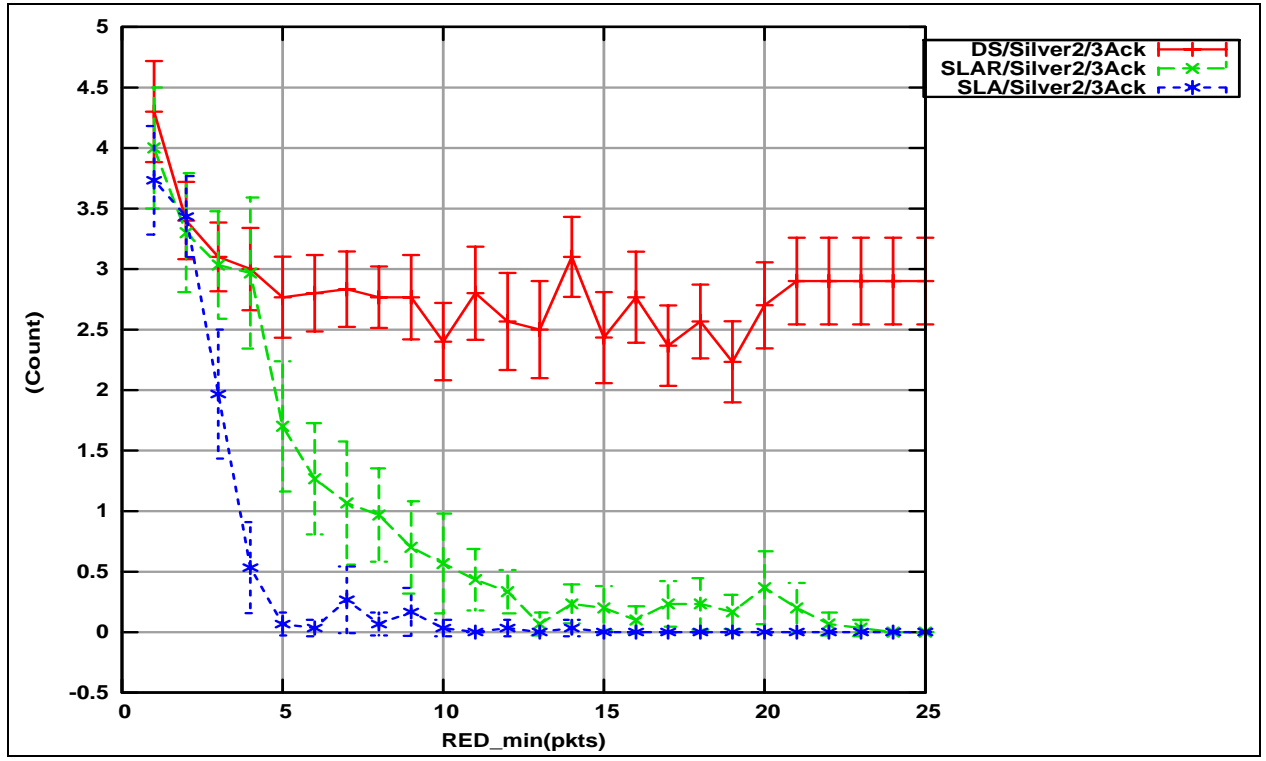

Figure 111 3+ duplicate ACKs Versus RED min threshold

To summarize this section's findings, we showed that the "bad" reordering is not aggravated as much as we expected as the lower class average queue size increase compared to the upper class average queue size. However, since the SLAR upgraded packets flow on the upper class pipe, small queue sizes in the upper class could result in deteriorated quality due to increased losses.

\subsection{Effect of leaky bucket initial size}

In sections 8.3 and 8.4 , we showed the effect of using a token bucket policer on TCP sources. TCP is a bursty protocol in nature, and the main problem of using such policer types is that they impose bursty losses (and thus poor performance) on TCP if the TCP burst does not fit in the configured CBS parameter. There are several papers that discuss the impact of 
token bucket policers on TCP and some even propose more TCP-friendly policers [87 (along with its referenced papers)].

In this section, we will not analyze the effect of CBS on TCP due to prior art as mentioned above, however we will briefly show the effect of the Gold CBS parameter for the various models. We will fix the CBR8 (Silver Background rate) value at 1,450 KBps (which we observed earlier as a high enough value to cause reordering in the SLAR model without congesting the network) while varying the Gold CBS. All other parameters are the same as in section 8.5 i.e. the Gold policer parameters set to [CBS=variable, $C I R=330,600]$, the Silver policer parameters set to $[\mathrm{CBS}=32,000, \mathrm{CIR}=230,600]$, and the Bronze policer parameters set to $[\mathrm{CBS}=32,000, \mathrm{CIR}=230,600]$.

Figure 112 and Figure 113 show Client2's Gold goodput versus the Gold CBS for TCP packet sizes of 1,000 Bytes and 1,500 Bytes, respectively. The corresponding maximum approximated TCP rates are 20x1,000/120 $=166 \mathrm{KBps}$, and 20x1,500/120 $=250 \mathrm{KBps}$ for the mentioned packet sizes.

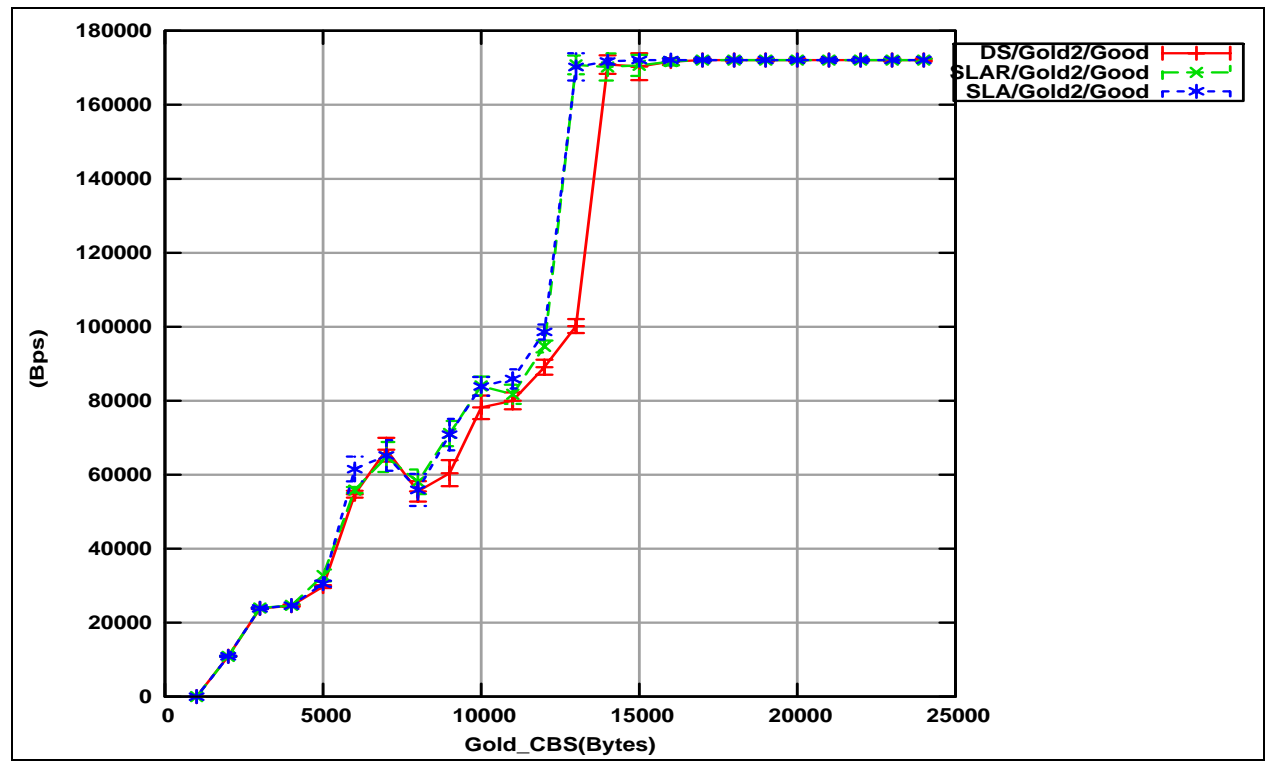

Figure 112 Gold TCP goodput Versus the Gold CBS (Packet size $=1,000 B$ ) 


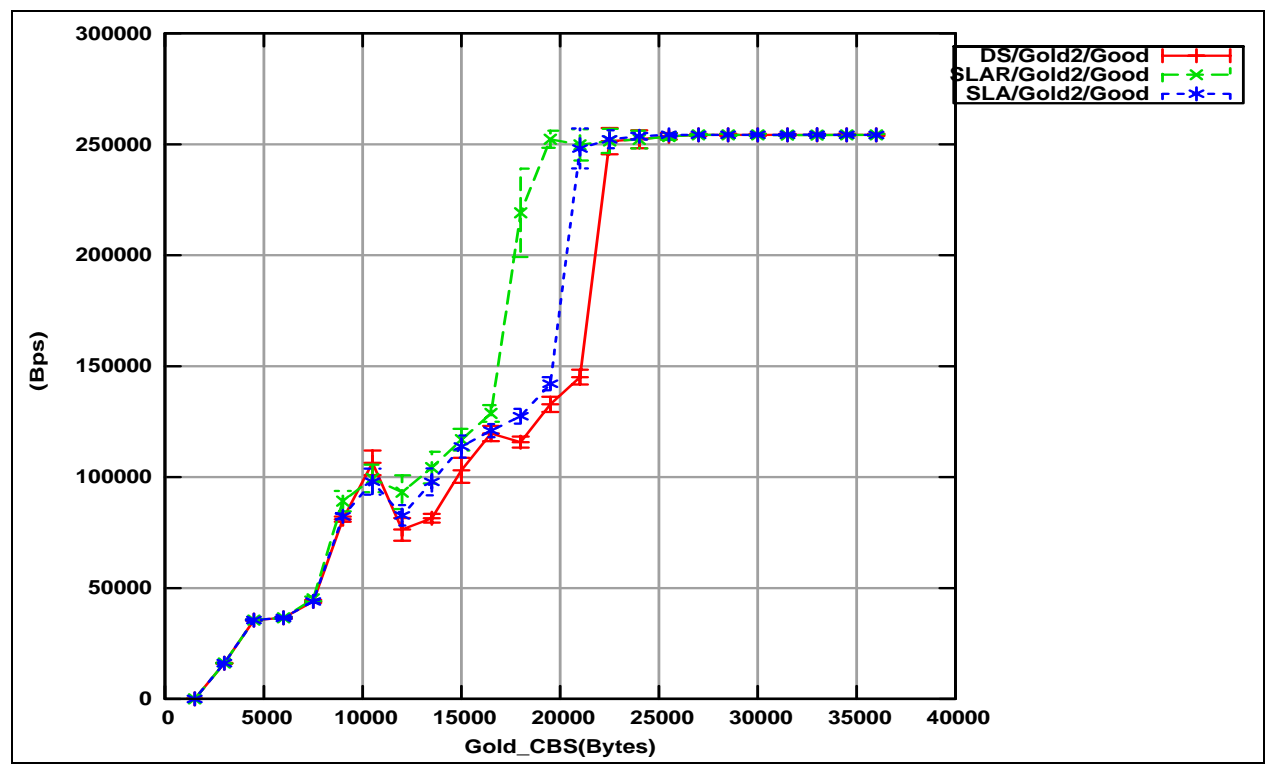

Figure 113 Gold TCP goodput Versus the Gold CBS (Packet size $=1,500 B$ )

Both figures show that the CBS parameter severely affects the TCP goodput as predicted (due to TCP burstiness). What is noticeable about the above figures is the similarity in the shape of the curves where the goodput increases, then we observe a small bump, then another increase, a jump and then a flat line. What could cause such bumps (at 7,000 Bytes for 1,000 Bytes packets and at 10,500 Bytes for 1,500 Bytes packets) and early saturations (flat line at 15,000Bytes for 1,000 Bytes packets and 22,500Bytes for 1,500 Bytes packets), given that the cwnd is equal to 20 packets (in NS-2, the cwnd is in units of packets) and that the full burst should have been 20x1,000=20,000Bytes for 1,000 Bytes packets and $20 \times 1,500=30,000$ Bytes for 1,500 Bytes packets?

Remember that TCP slow start is actually an exponential curve, where the window size doubles every RTT (first packet is sent, upon ACK reception 2 packets are sent, upon both ACK reception, 4 packets are sent and so on). It seems that the bumps coincide with $\mathrm{CBS}=7$ packet-sizes $(7 \times 1,000=7,000$ and $7 \times 1,500=10,500)$ and that the early saturations coincide with $\mathrm{CBS}=15$ packet-sizes $(15 \times 1,000=15,000$ and $15 \times 1,500=22,500)$. In fact, 7 is 
the sum of 1,2, and 4, and 15 is the sum of 1,2, 4 and 8 . Notice also in the graphs that there is a small bump at $\mathrm{CBS}=3,000$ Bytes in Figure 112 and at $\mathrm{CBS}=4,500$ Bytes in Figure 113, both corresponding to the sum of 1 plus 2 ( $=3$ packet-sizes). So it seems that as CBS increases we have some preferred values corresponding to the sum of the discrete exponential series starting at 1 ; these values being $3(1+2), 7(1+2+4)$, and $15(1+2+4+8)$. As explained earlier, the slow start property of TCP triggered upon timeout detection of losses causes this exponential increase in window size.

Figure 114 shows Client2's Silver TCP goodput versus the Gold CBS. The figure shows that the Silver TCP goodput takes advantage of upgrades for the SLAR and SLA cases as soon as there is at least 2 packet sizes worth of tokens $(2 \times 1,500=3,000$ Bytes $)$. The Gold token refresh rate being at 330KBps, the Gold bandwidth gap of 330KBps minus the Goldrate as per Figure 113 for a particular Gold CBS value was enough for the Silver to get the upgrade needed to reach the approximated maximum TCP rate of $250 \mathrm{KBps}$.

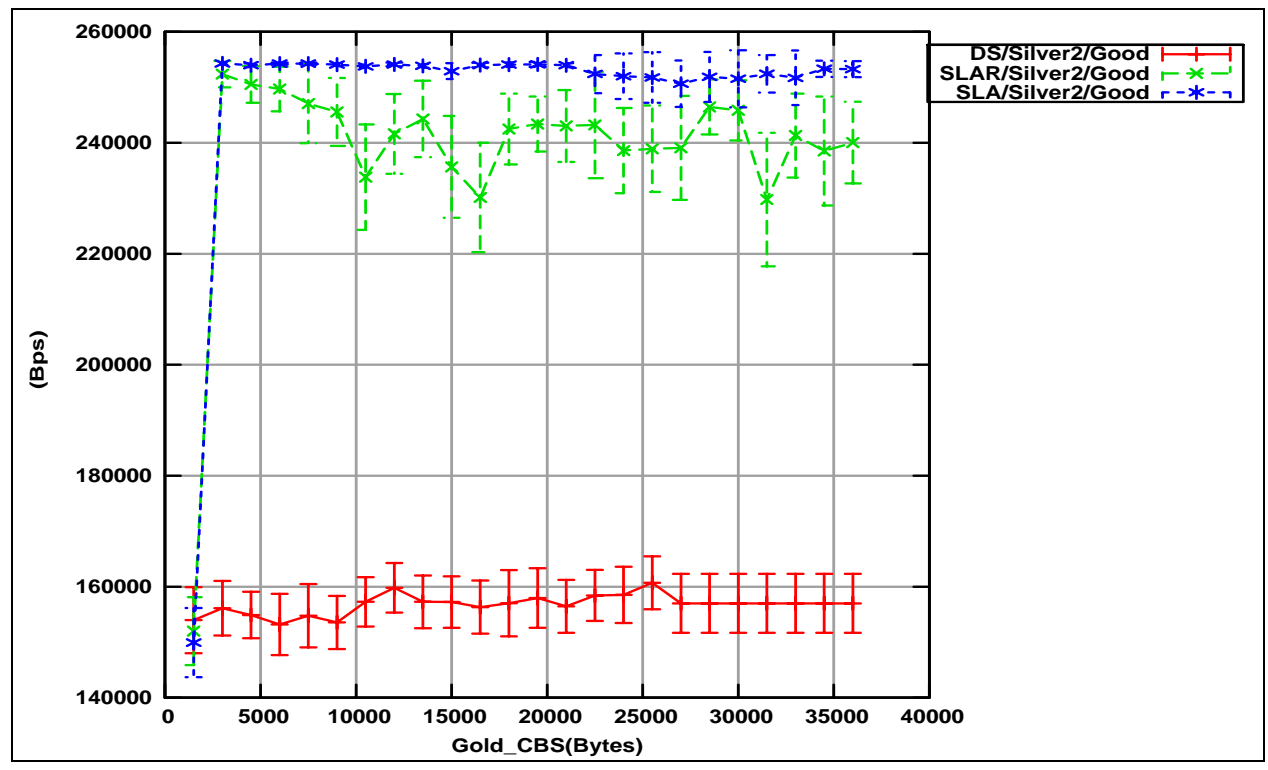

Figure 114 Silver TCP goodput Versus the Gold CBS (Packet size $=1,500 B$ ) 
To summarize this section's findings, we showed that the CBS value severely impacts TCP performance, as observed in several prior art, due to limiting the TCP burst size. We also showed that TCP prefers some CBS values, corresponding to the sum of the discrete exponential series $\left(\operatorname{Sum}\left(2^{\mathrm{n}}\right)\right.$, e.g. $\left.3,7,15\right)$, due to the exponential slow start behavior upon timeout detection of packet loss. Finally, we observed that it doesn't require a large upper class CBS value for the lower class to benefit from upgrades (an upper CBS value of 2 packets was enough).

\subsection{Effect of Round Trip Time}

In the previous sections, we used a propagation delay of $10 \mathrm{~ms}$ over all links in the network. We also said that the maximum TCP throughput is approximated at cwnd/RTT (or $20 \times 1500 /(2 \times 6 \times 10 \mathrm{~ms})=250 \mathrm{KBps}$. In this section, we show the Round Trip Time (RTT) effect on the TCP throughput for the various models by varying the per-link propagation delay.

Figure 115 shows Client2's Gold TCP goodput versus the per-link propagation delay. The minimum RTT is estimated at $2 \times 6 \times 10 \mathrm{~ms}=120 \mathrm{~ms}$ (ignoring transmission, queuing and processing delays); this is the time it takes the packet to traverse 6 links in the forward and backward direction. The figure shows that when the propagation delay is small, the TCP goodput is lower than expected, then it peaks out at $8 \mathrm{~ms}$ to drop back down as the propagation delay is further increased. Remember that we are policing Client2's Gold traffic at 330KBps, and thus even though with a smaller RTT the TCP throughput should be higher, the policer keeps the TCP throughput below the CIR. 


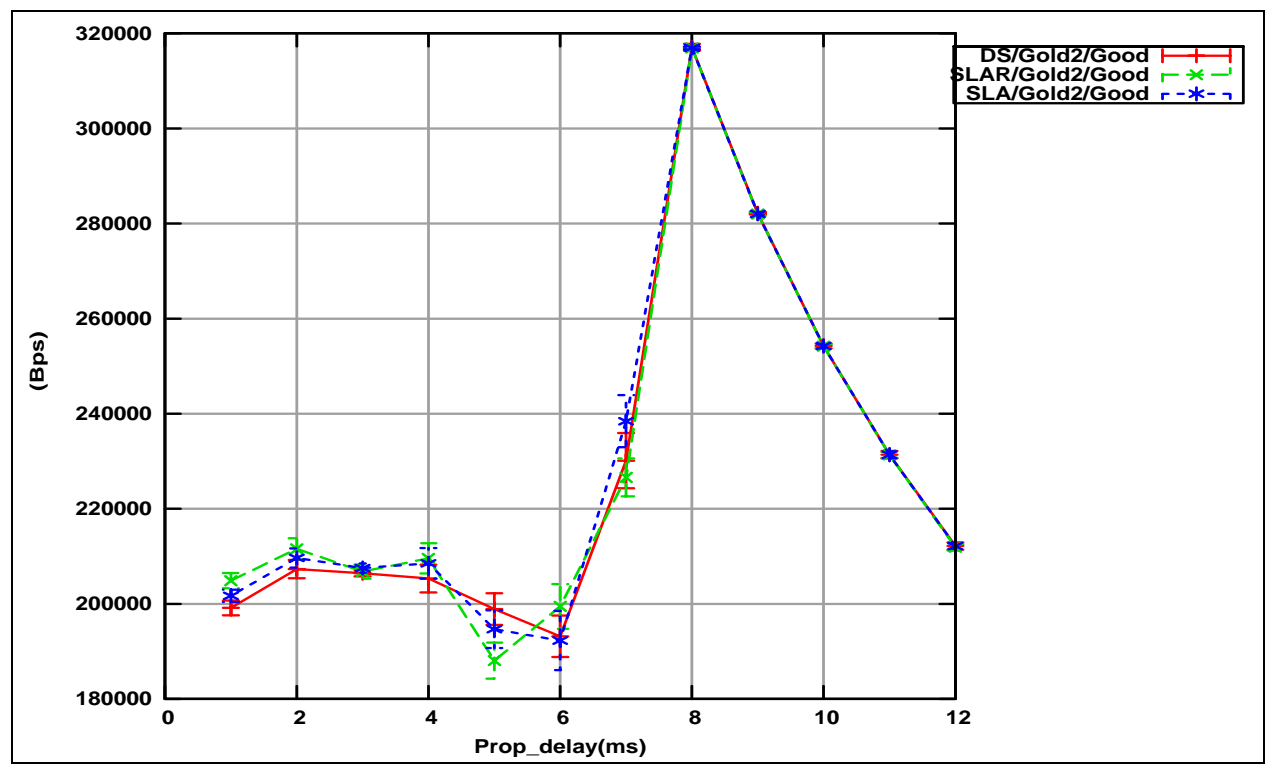

Figure 115 Gold TCP Goodput Versus the per link propagation delay

Figure 116 shows the same data as Figure 115, except that we add the theoretical approximated maximum TCP throughput for RTT $=2 \times 6 \times$ Prop_delay labeled min_RTT, the estimated RTT with an extra 10ms delay (due to queuing/transmission for example) labeled RTT_10 and the policing rate at ingress labeled CIR. The min_RTT and the RTT_10 curves show the approximated TCP throughput had we not policed on ingress for 2 different values of RTT. From this figure, we can see that when the per-link propagation delay is smaller than $8 \mathrm{~ms}$, the RTT is small enough to result in a TCP throughput higher than the policed rate. Which means that, for propagation delay values of less than $8 \mathrm{~ms}$, the TCP Gold traffic has observed drops, due to policing, and that these drops forced TCP into congestion avoidance (thus lowering its rate). As the RTT is increased, the TCP throughput is decreased resulting in lower packet drops due to policing (Figure 117 shows the drops due to policing). For a per-link propagation delay of $8 \mathrm{~ms}$ or more, the TCP maximum throughput becomes smaller than the policed rate and all three models throughput match the approximated theoretical 
curve (labeled min_RTT in the graph). This explains the behavior in Figure 115 where the goodput peaks out at $8 \mathrm{~ms}$ and then drops down.

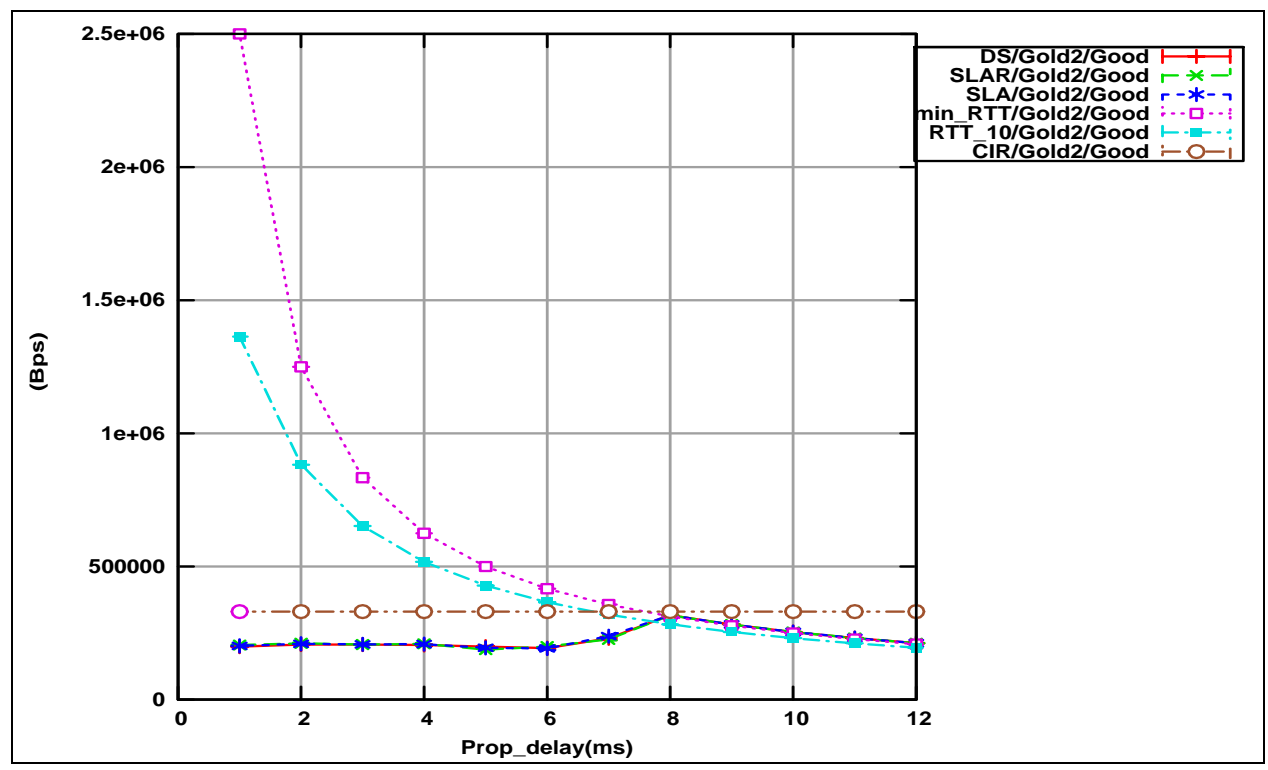

Figure 116 Max Theoretical TCP goodput Versus the per link propagation delay

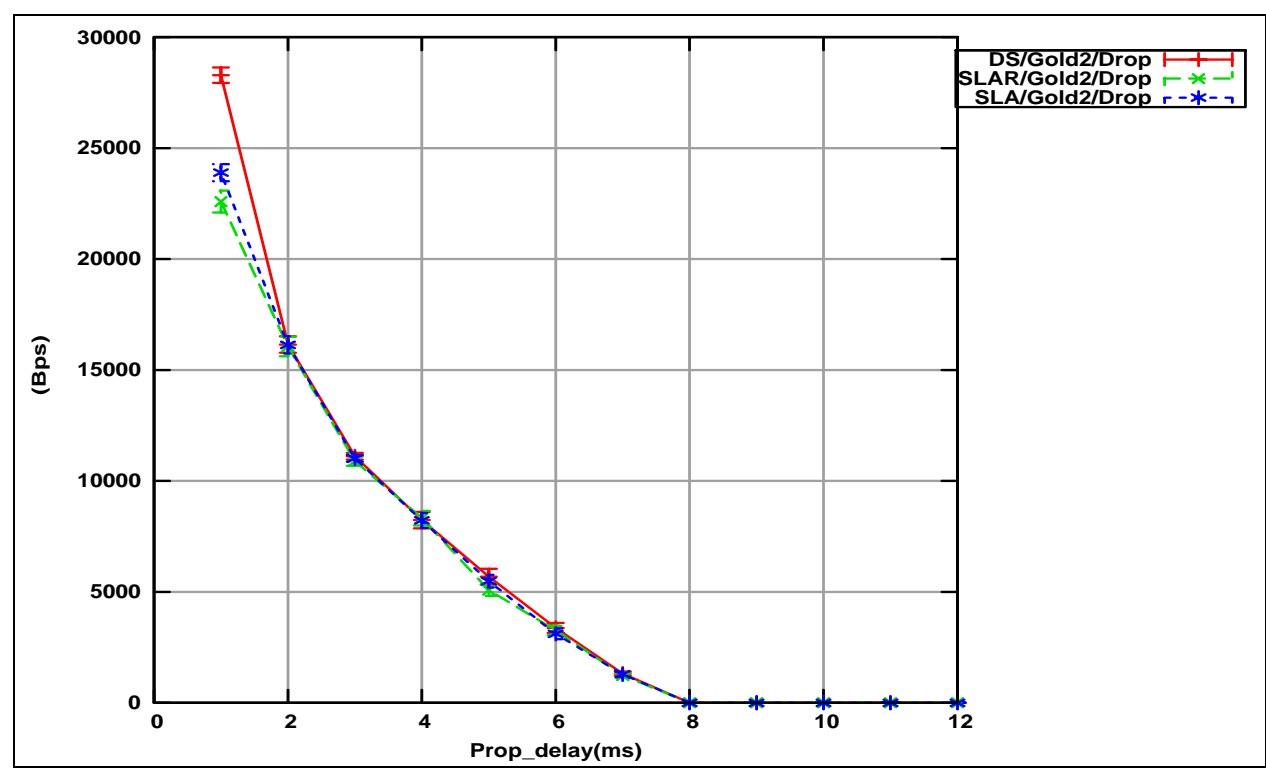

Figure 117 Gold ingress drop rate due to policing Versus the per link propagation delay

Figure 115 shows that if the RTT is over- or under-estimated, then the customer would be wasting some of his/her paid-for reserved bandwidth. For the customer to fully use 
his/her paid-for reserved bandwidth, s/he needs to accurately estimate the RTT. Or in other words, if the customer does not know the bandwidth he needs to purchase from a provider, the most efficient bandwidth value (efficient in terms of utilization) would be dictated by an accurate estimation of the RTT. In general, the TCP RTT can not be estimated a priori unless the end-points within the network are predefined; Even if the end-points are defined, the network queuing, routing, etc. behavior is not always deterministic. So, could the automatic upgrades SLA benefit from such a TCP characteristic?

Figure 118 shows Client2's Silver TCP goodput versus the per link propagation delay (we also left the DS Gold goodput for convenience, the SLAR and SLA Gold goodput being the same as per Figure 115 - we don't upgrade the Gold traffic). Note that both the SLAR and SLA models show that they indeed benefited from holes created by a "bad" estimate of RTT when compared to the DS model. Remember that we are policing the Gold at 330KBps, and that for a per-link propagation delay of less than $8 \mathrm{~ms}$, the Gold TCP could not reach the CIR rate due to ingress dropping. However, the Gold gap generated by this TCP characteristic was used by the Silver traffic to get higher throughput (the Silver traffic was policed at 230KBps; for the DS case, for a small RTT, the Silver traffic was dropped due to ingress policing resulting in a TCP rate much less than $230 \mathrm{KBps})$. At $8 \mathrm{~ms}$, the Gold gap is at its minimum since the Gold traffic is almost completely using the reserved Gold bandwidth and thus both the SLAR and SLA dip to minimum upgrades (as also shown in Figure 119). For a propagation delay higher than $8 \mathrm{~ms}$, the RTT becomes large enough to make the maximum TCP rate less than the CIR as already mentioned, and thus the SLAR and SLA models use this gap to upgrade Silver packets. 


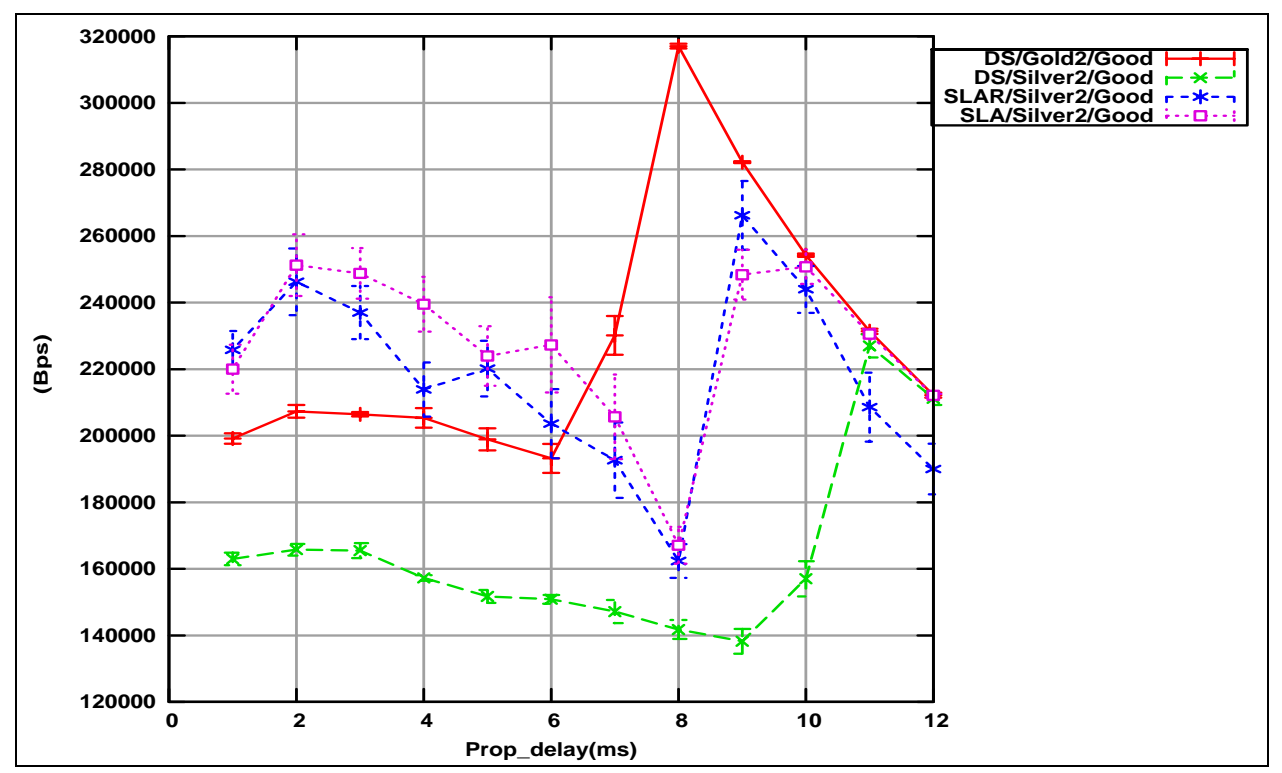

Figure 118 Silver TCP Goodput Versus the per link propagation delay

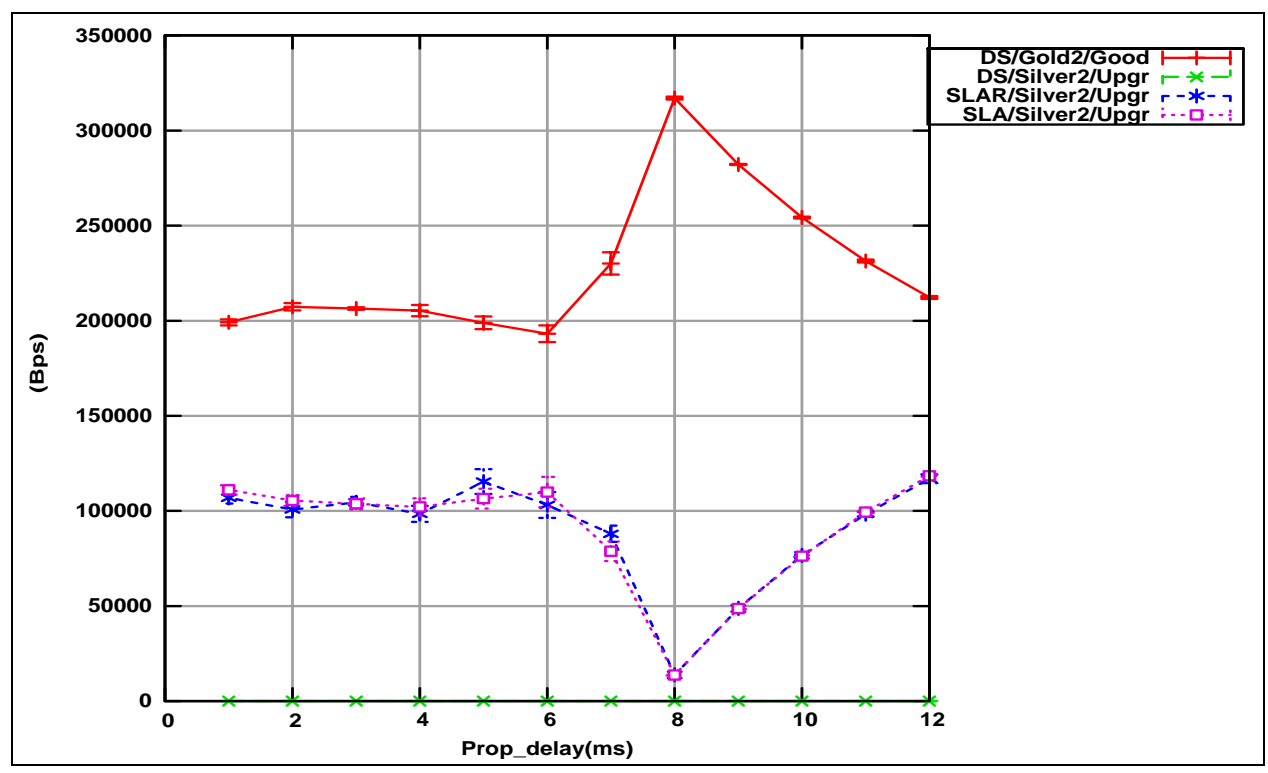

Figure 119 Silver TCP Upgrade rate Versus the per link propagation delay

As a summary to this section, we showed that in the DS model, for the client bought bandwidth to be fully utilized, it needs to be derived from an accurate estimation of the RTT. Since in a network a client is not necessarily communicating with the same endpoint, and since the RTT depends on the state of the network for a specific endpoint, the bandwidth 
bought can be severely underutilized. We also showed that the SLAR and the SLA models can use the gap generated from such a TCP behavior to upgrade lower-class packets to fill that gap, resulting in a much better utilization of the paid-for reserved bandwidth.

\subsection{Effect of TCP type}

So far, we have been using TCP Reno in our simulations. In this section, we investigate whether the TCP flavor has any impact on the SLA. For this matter, we compare the TCP Reno, Tahoe and SACK behaviors using the NS-2 provided implementations.

The NS-2 "Tahoe" TCP agent, Agent/TCP, performs congestion control and roundtrip-time estimation in a way similar to the version of TCP released with the 4.3BSD "Tahoe" UNIX system release from UC Berkeley. The congestion window is increased by one packet per new ACK received during slow-start and is increased by $1 / \mathrm{w}$ for each new ACK received during congestion avoidance. The NS-2 Reno TCP agent is very similar to the Tahoe TCP agent, except it also includes fast recovery, where the current congestion window is "inflated" by the number of duplicate ACKs the TCP sender has received before receiving a new ACK. A "new ACK" refers to any ACK with a value higher than the highest seen so far. In addition, the Reno TCP agent does not return to slow-start during a fast retransmit. Rather, it sets the congestion window to half the current window. Finally, the NS-2 SACK TCP agent (RFC2018) implements selective repeat based on selective ACKs provided by the receiver.

Figure 120, Figure 121, and Figure 122 show Client2's Gold, Silver and Bronze TCP goodput, respectively, for the various TCP flavors under the SLA model. As shown, the SLA model is agnostic to the TCP flavor, except when there are some losses (Bronze case) where 
both the Reno and SACK perform a bit better due to fast retransmit or SACK (note that when a timeout occurs, Reno behaves similarly to Tahoe). Note that the confidence intervals appear to be wide in Figure 120, however when looking at the y-axis scale they are actually very narrow.

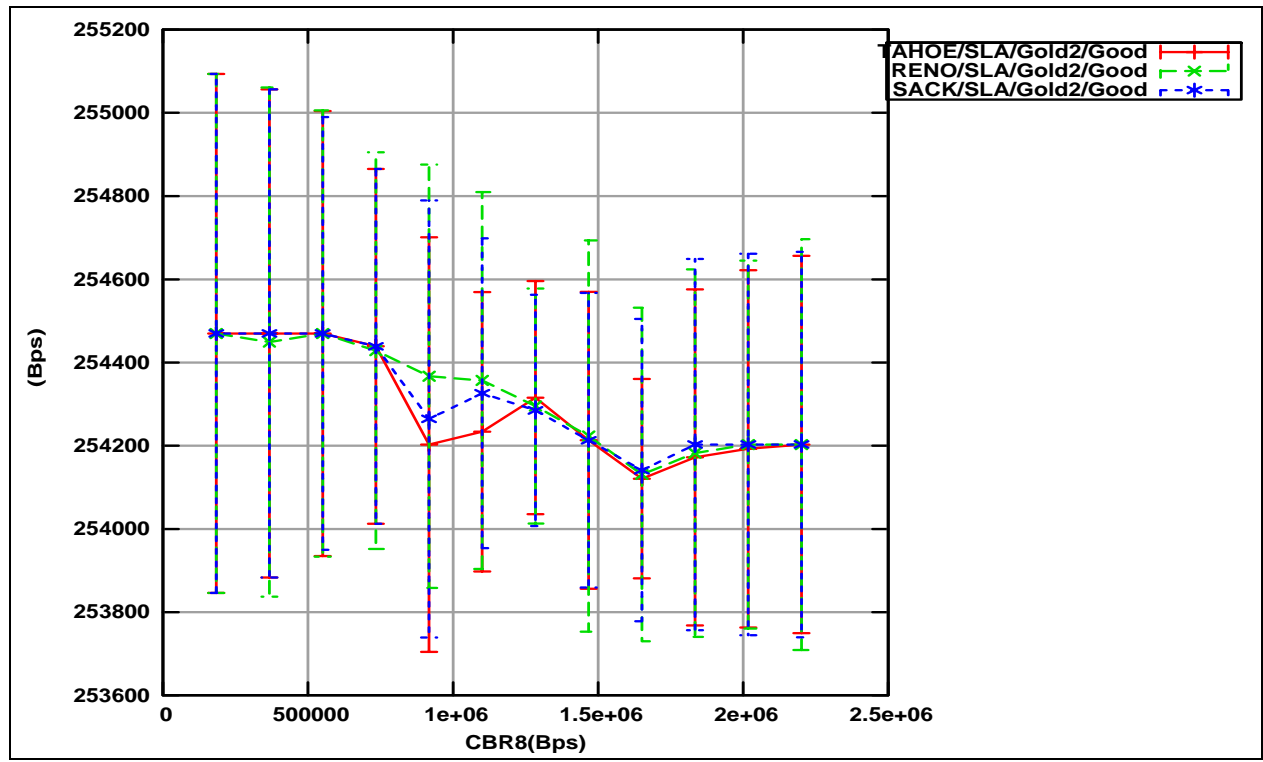

Figure 120 SLA Gold TCP goodput Versus Silver Background rate

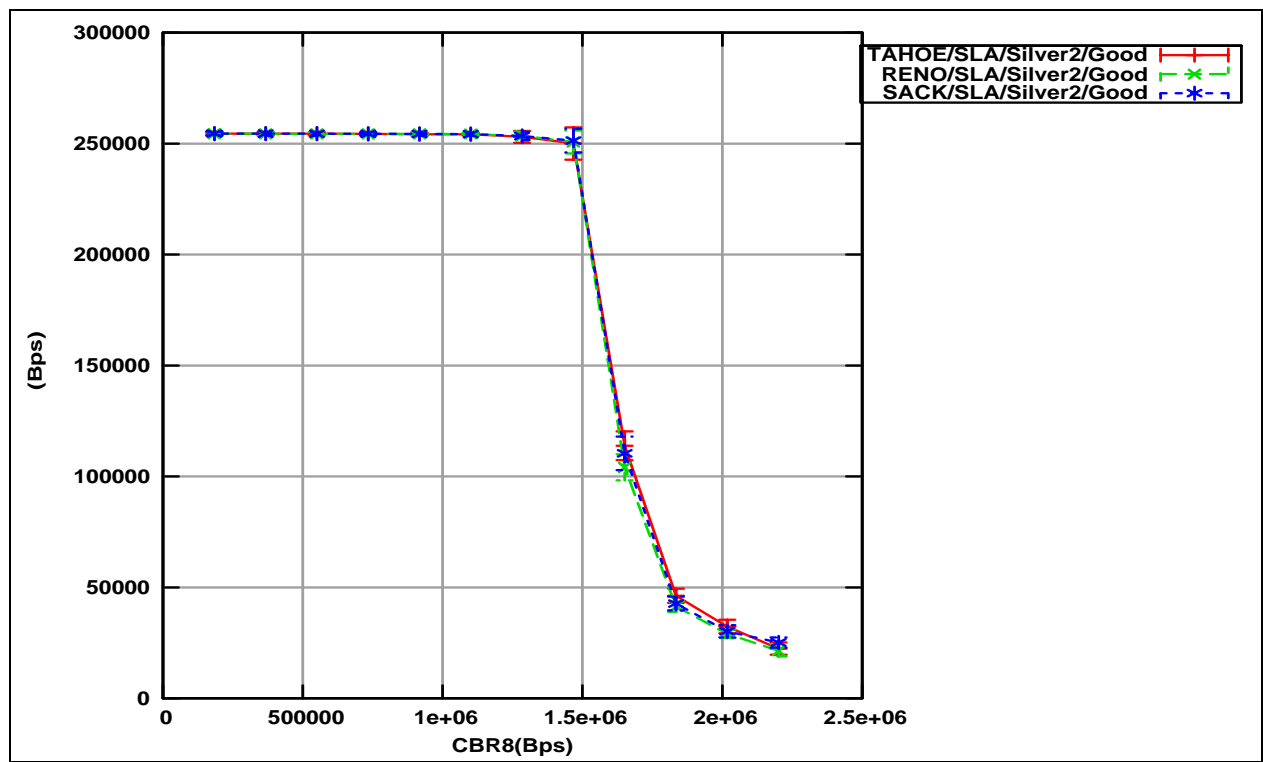

Figure 121 SLA Silver TCP goodput Versus Silver Background rate 


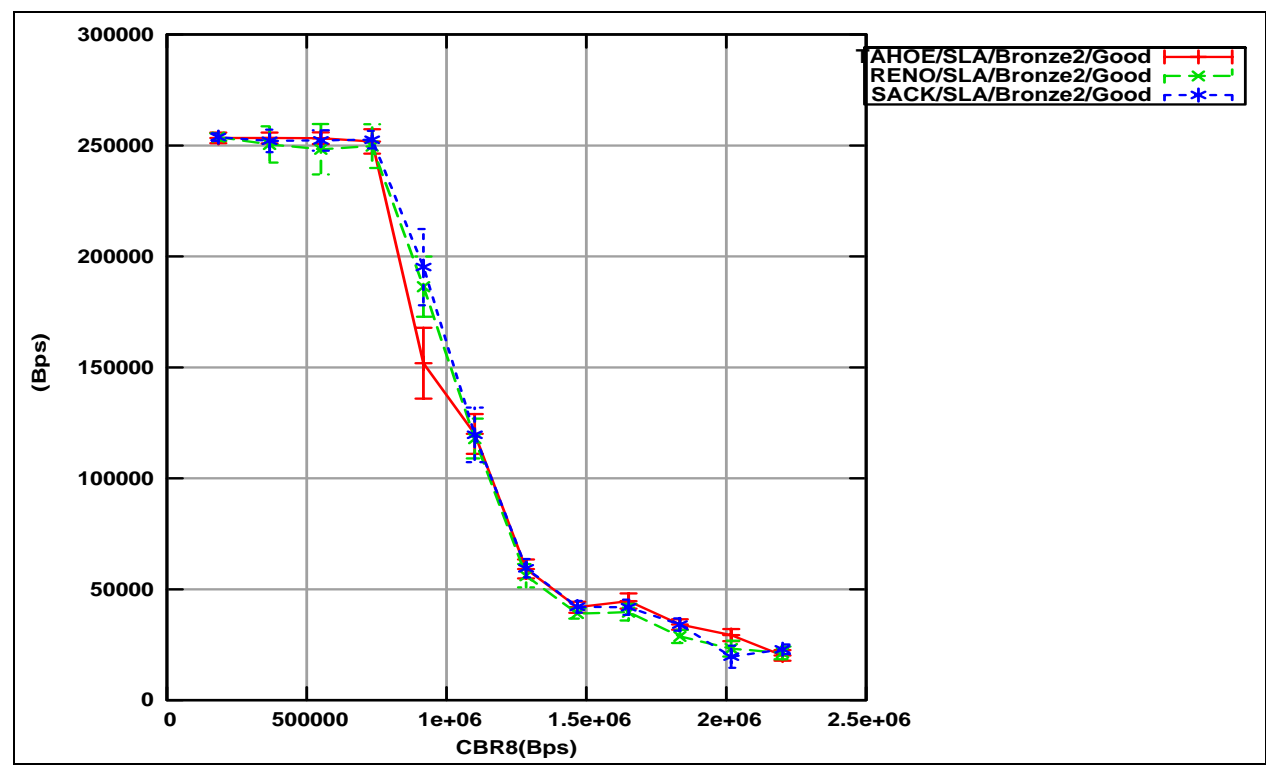

Figure 122 SLA Bronze TCP goodput Versus Silver Background rate

Figure 123 and Figure 124 show the Silver and Bronze goodput for the SLAR model. The figures show similar behavior for all TCP flavors except in the case of congestion where SACK has the best goodput.

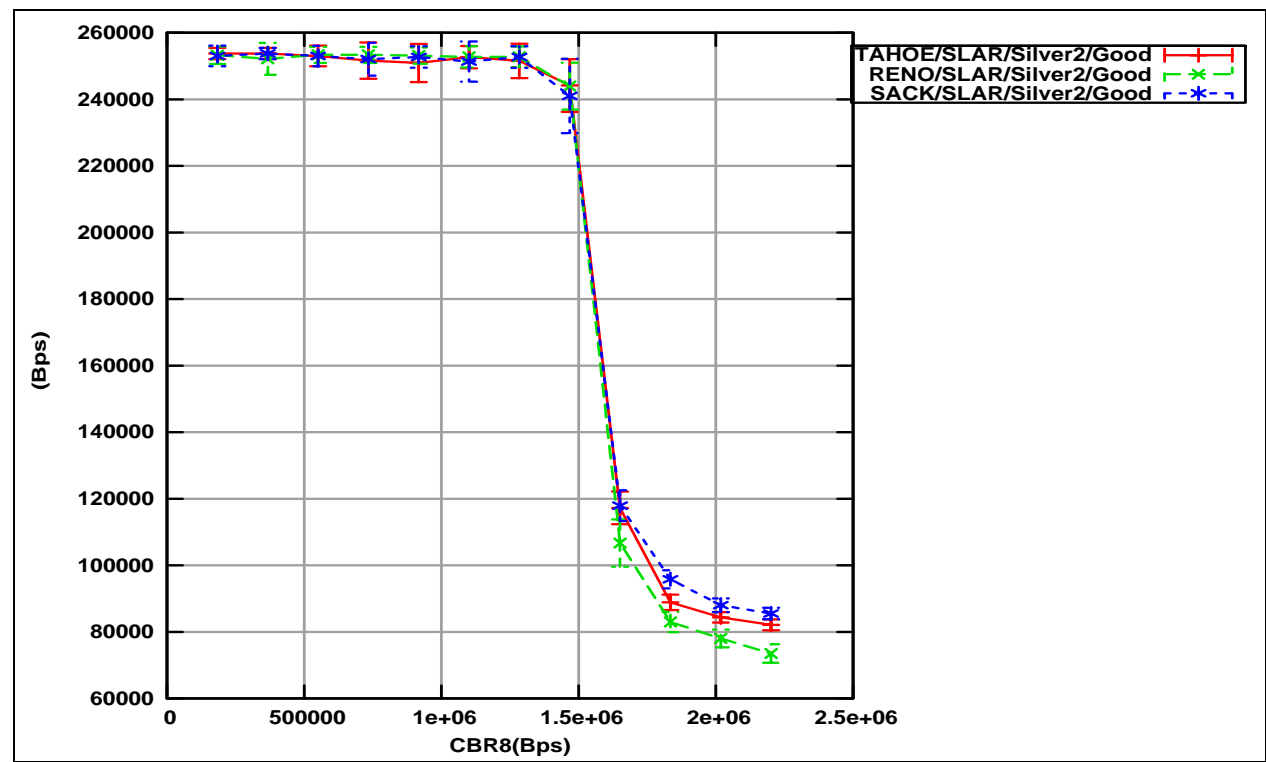

Figure 123 SLAR Silver TCP goodput Versus Silver Background rate 


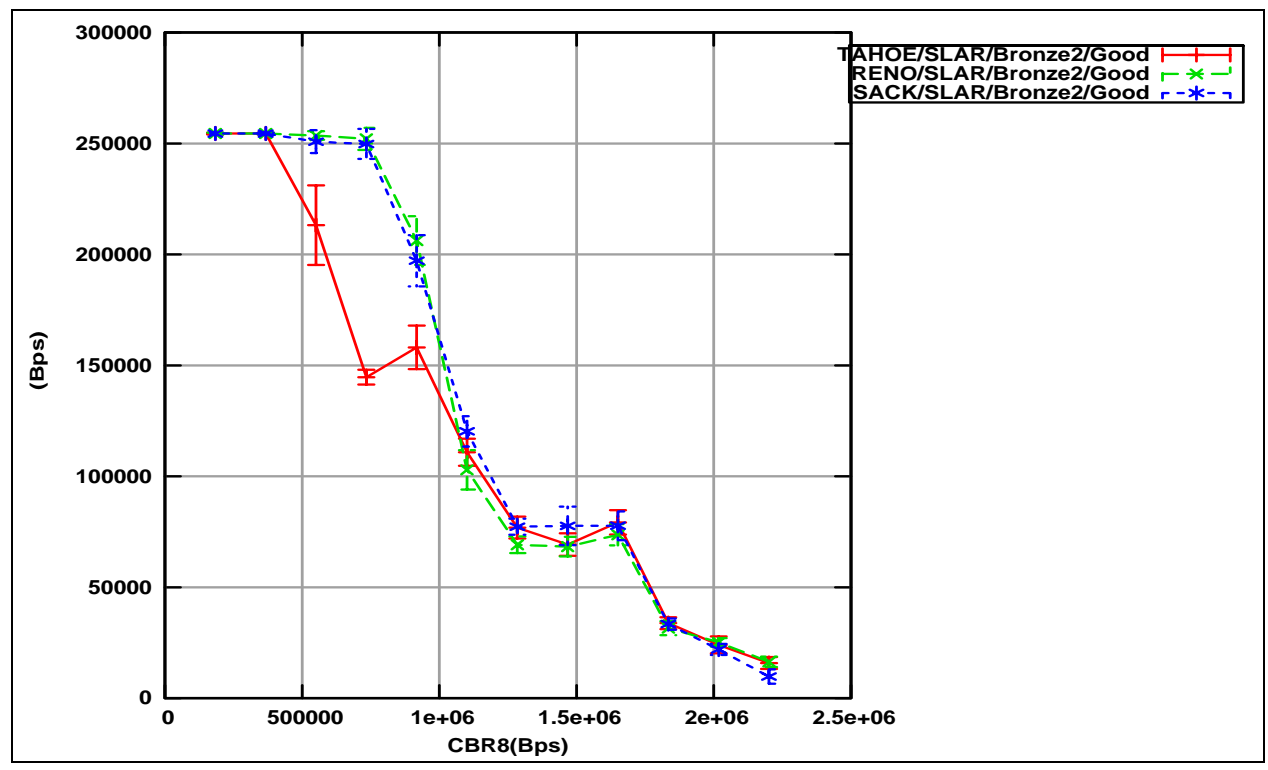

Figure 124 SLAR Bronze TCP goodput Versus Silver Background rate

As a summary to this section, we showed that the TCP flavor has little to no effect on the TCP performance in the SLA model, whereas in the SLAR model, TCP SACK showed a little improvement upon congestion.

\subsection{Summary and Observations}

In this chapter, we analyzed the TCP behavior for the SLA model comparing it with the DS and SLAR models. We highlighted TCP QoS related issues such as the effect of tight ingress policing which causes lower TCP rates than the expected policed rates, and the effect of the Committed Burst Size (CBS) which limits the TCP rates by limiting the TCP burstiness. We highlighted how lower-class TCP sources benefits from the automatic upgrades (SLAR and SLA) to achieve higher throughput when compared to the non-upgrades model (DS), and that the reordering effect caused by the SLAR model is dampened by the TCP receive buffers. We observed that the SLAR model behaves better than the SLA model under heavy congestion of the lower class pipe, since the upgraded packets flow on the upper 
pipe bypassing the congestion in the lower pipe versus the SLA model where upgraded packets flow on the lower class pipe to maintain the order. We showed that the TCP throughput is bounded by a maximum rate approximated by cwnd/RTT and that TCP can not make use of upper-class gaps larger than this maximum rate. We also showed that in the DS model, for a client to fully utilize his/her paid-for bandwidth, s/he needs to accurately estimate the RTT in order to avoid tight policing (RTT over-estimated) and over-booking (RTT under-estimated), whereas the SLAR and SLA models provide full usage of the paidfor bandwidth by upgrading lower class traffic to use the gap in the upper class. Finally, we concluded that the TCP flavor has little to no impact on the upgrade (SLAR or SLA) benefits. 


\section{Chapter 9}

\section{Experimental Results -}

\section{Generalizing into N-Class SLA}

In the previous chapters, we have proposed a 3-tier SLA with automatic class upgrades incorporating a reordering solution to prevent packet reordering due to upgrades. We showed how lower classes can benefit from upper-class gaps to get better throughput without affecting the promised QoS characteristics of the upper classes. In this section, we will show that the proposed solution does not depend on the number of priority classes, and that the number of priority classes can be generalized for $\mathrm{N}$ traffic classes, so that when there is a gap in priority $\mathrm{x}$ class, priority y classes where $\mathrm{y}>\mathrm{x}$ could benefit from that gap, by showing simulation results for $\mathrm{N}=8$.

We start by describing the simulation configuration in section 9.1 . We then show in sections 9.2 and 9.3 how upgrades are handled in the 8 traffic classes' case for both UDP and TCP traffic types, and finally, we summarize this chapter's findings in section 9.4. 


\subsection{Simulation Configuration}

\subsubsection{Topology}

We first start by describing the topology used in this scenario, also shown in Figure

125.

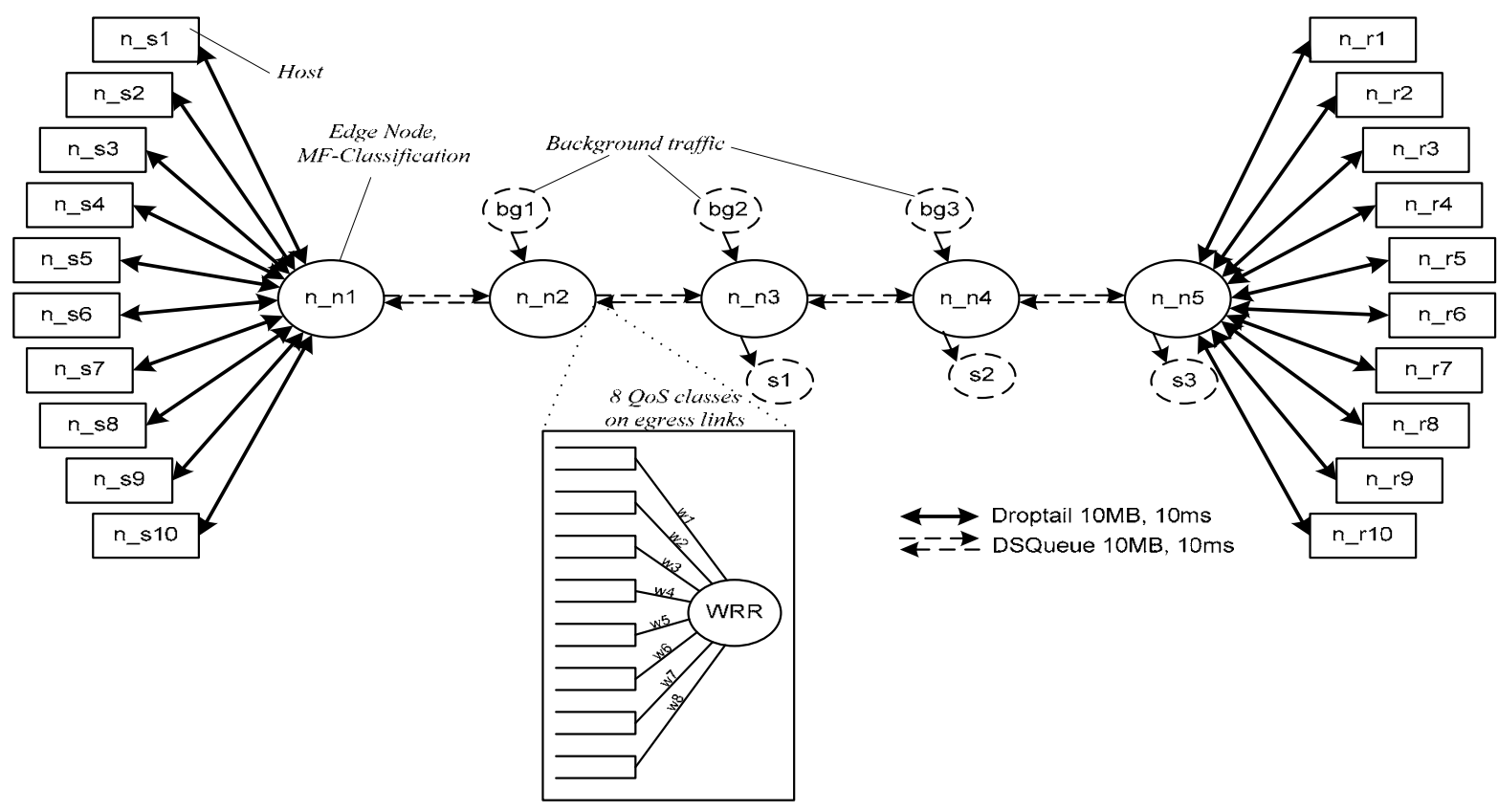

Figure 125 Network topology

The topology consists of 10 source hosts (or clients) labeled n_s1 through n_s10, 10 corresponding receiving hosts labeled n_r1 through $\mathrm{n} \_r 10$, and 5 internal nodes labeled n_n1 through n_n5. 2 of the 10 clients (client1 and client2) will assume the 8-tier SLA and the other 8 clients (n_s3 through n_s10) will act as background traffic sources each with a particular traffic class ( 1 through 8 , respectively). The number of receiving hosts matches the number of sources, so each host sinks its corresponding client's traffic.

RED will be configured on each of the traffic classes inside the network, except for class 1 which is over-provisioned and designed for real-time traffic. 


\subsubsection{Network setup}

In this scenario we assume all links have a capacity of $10 \mathrm{MBps}$ and a propagation delay of $10 \mathrm{~ms}$. The network capacity is partitioned so that each of the traffic classes' ratio is 112 except for the highest priority class which has a ratio of 216 to allow for overprovisioning. These ratios were chosen to represent a provider's initial dimensioning of the network.

Table 10 and Table 11 summarize the various parameters and values used in this scenario. Notice that all background traffic classes rates were set to $162,500 \mathrm{Bps}$ (omitted for brevity). Notice also in Table 11 that the per node RED parameters values were omitted since they are the same $(\mathrm{N}(\mathrm{i})-\mathrm{N}(\mathrm{j})$ is for $\mathrm{j}=\mathrm{i}+1, \mathrm{i}=2,3,4)$. 
Table 10 Network Parameters, 8-classes Scenario

\begin{tabular}{|c|c|c|c|c|c|c|c|c|c|}
\hline Client & Class & \begin{tabular}{|l|} 
Traffic \\
type
\end{tabular} & $\begin{array}{l}\text { Rate } \\
\text { (Bps) }\end{array}$ & $\begin{array}{l}\text { Pkt Sz } \\
\text { (B) }\end{array}$ & $\begin{array}{l}\text { LB CBS } \\
\text { (B) }\end{array}$ & $\begin{array}{l}\text { LB CIR } \\
\text { (Bps) }\end{array}$ & \begin{tabular}{|l|} 
FS \\
WRR \\
\end{tabular} & \begin{tabular}{|l|} 
SS \\
WRR \\
\end{tabular} & GSR \\
\hline Client1 & 1 & UDP CBR & 20000 & 500 & 5000 & 100000 & 100 & \begin{tabular}{|l|}
80 \\
\end{tabular} & 100000 \\
\hline Client1 & 2 & UDP CBR & 140000 & 500 & 5000 & 100000 & 100 & 80 & 100000 \\
\hline Client1 & 3 & UDP CBR & 140000 & 500 & 5000 & 100000 & 100 & 80 & 100000 \\
\hline Client1 & 4 & UDP CBR & 140000 & 500 & 5000 & 100000 & 100 & 80 & 100000 \\
\hline Client1 & 5 & UDP CBR & 20000 & 500 & 5000 & 100000 & 100 & 80 & 100000 \\
\hline Client1 & 6 & UDP CBR & 140000 & 500 & 5000 & 100000 & 100 & 80 & 100000 \\
\hline Client1 & 7 & UDP CBR & 140000 & 500 & 5000 & 100000 & 100 & 80 & 100000 \\
\hline Client1 & 8 & UDP CBR & 140000 & 500 & 5000 & 100000 & 100 & 80 & 100000 \\
\hline Client2 & 1 & TCP FTP & Dynamic & $<1500$ & 32000 & 330000 & 33 & 194 & 330000 \\
\hline Client2 & 2 & TCP FTP & Dynamic & $<1500$ & 32000 & 230000 & 23 & 194 & 230000 \\
\hline Client2 & 3 & TCP FTP & Dynamic & $<1500$ & 32000 & 230000 & 23 & 194 & 230000 \\
\hline Client2 & 4 & TCP FTP & Dynamic & $<1500$ & 32000 & 230000 & 23 & 194 & 230000 \\
\hline Client2 & 5 & TCP FTP & Dynamic & $<1500$ & 32000 & 230000 & 23 & 194 & 230000 \\
\hline Client2 & 6 & TCP FTP & Dynamic & $<1500$ & 32000 & 230000 & 23 & 194 & 230000 \\
\hline Client2 & 7 & TCP FTP & Dynamic & $<1500$ & 32000 & 230000 & 23 & 194 & 230000 \\
\hline Client2 & 8 & TCP FTP & Dynamic & $<1500$ & 32000 & 230000 & 23 & 194 & 230000 \\
\hline Client3 & 1 & UDP CBR & 500000 & 500 & 5000 & 782000 & 100 & 173 & 1730000 \\
\hline Client4 & 2 & UDP CBR & 762500 & 500 & 5000 & \begin{tabular}{|l|l}
782000 \\
\end{tabular} & 100 & 79 & 790000 \\
\hline Client5 & 3 & UDP CBR & 775000 & 500 & 5000 & 782000 & 100 & 79 & 790000 \\
\hline Client6 & 4 & UDP CBR & 775000 & 500 & 5000 & 782000 & 100 & 79 & 790000 \\
\hline Client7 & 5 & UDP CBR & 787500 & 500 & 5000 & 782000 & 100 & 79 & 790000 \\
\hline Client8 & 6 & UDP CBR & 787500 & 500 & 5000 & \begin{tabular}{|l}
782000 \\
\end{tabular} & 100 & 79 & 790000 \\
\hline Client9 & 7 & UDP CBR & 800000 & 500 & 5000 & 782000 & 100 & 79 & 790000 \\
\hline Client10 & 8 & UDP CBR & 800000 & 500 & 5000 & 782000 & 100 & 79 & 790000 \\
\hline bg1 & $x$ & UDP CBR & 162500 & 500 & & & & & \\
\hline bg2 & $x$ & UDP CBR & 162500 & 500 & & & & & \\
\hline bg3 & $x$ & UDP CBR & 162500 & 500 & & & & & \\
\hline
\end{tabular}


Table 11 Nodes configuration, 8 classes scenario

\begin{tabular}{|l|l|l|l|l|l|l|}
\hline Node & Class & RED A & RED B & RED P & WRR & GSR \\
\hline$n(i)-n(j)$ & 1 & inf & inf & 0 & 216 & 2160000 \\
\hline$n(i)-n(j)$ & 2 & 10 & 20 & 0.5 & 112 & 1120000 \\
\hline$n(i)-n(j)$ & 3 & 15 & 25 & 0.7 & 112 & 1120000 \\
\hline$n(i)-n(j)$ & 4 & 20 & 30 & 0.7 & 112 & 1120000 \\
\hline$n(i)-n(j)$ & 5 & 25 & 35 & 0.7 & 112 & 1120000 \\
\hline$n(i)-n(j)$ & 6 & 30 & 40 & 0.7 & 112 & 1120000 \\
\hline$n(i)-n(j)$ & 7 & 35 & 45 & 0.7 & 112 & 1120000 \\
\hline$n(i)-n(j)$ & 8 & 40 & 50 & 0.7 & 112 & 1120000 \\
\hline
\end{tabular}

\subsection{Handling Upgrades}

In the previous chapters, we showed how lower classes can benefit from upper-class gaps to get better throughput without affecting the promised QoS characteristics of the upper classes. In this section, we assume 8 priority classes (we will name them $\mathrm{p} 1$ through $\mathrm{p} 8, \mathrm{p} 1$ being the "best" quality of service class) to show that the automatic upgrades do not depend on the number of priority classes, and that when there is a gap in priority $\mathrm{x}$ class, priority $\mathrm{y}$ classes where $y>x$ could benefit from that gap. For that, we will vary clientl's priority 5 rate from 0 to 120,000 Bps to show how the lower priority classes can benefit from gaps in the priority 5 class.

Figure 126 shows the SLA behavior for client1's first 5 priority classes' goodput as the priority 5 traffic rate is changed (the label sx-py identifies source $\mathrm{x}$ (or client $\mathrm{x}$ ) and priority y). As seen in the figure, the top 4 priorities are not affected by priority 5 . Note that since the priority 1 rate is $20,000 \mathrm{Bps}$ and that it is being policed at $100,000 \mathrm{Bps}$ creating a gap of $80,000 \mathrm{Bps}$, priority 2 traffic is benefiting from that gap to get higher throughput than the policed rate (in fact 80,000 Bps of priority 2 traffic are being upgraded to priority 1 ). With 80,000 Bps priority 2 traffic being upgraded, the remaining 60,000 (priority 2 ingress 
rate is $140,000 \mathrm{Bps}$ ) are found conformant to the priority 2 policers (and that is why the priority 2 goodput is $140,000 \mathrm{Bps}$ versus the policed 100,000 Bps). In turn, the $60,000 \mathrm{Bps}$ priority 2 traffic generates a gap of 40,000 Bps which priority 3 makes use of. Since priority 3 ingress rate is $140,000 \mathrm{Bps}$ it does not leave any gap at priority 3 , and priority 4 traffic gets the policed rate of $100,000 \mathrm{Bps}$ goodput (the extra 40,000 Bps are policed as excess and dropped).

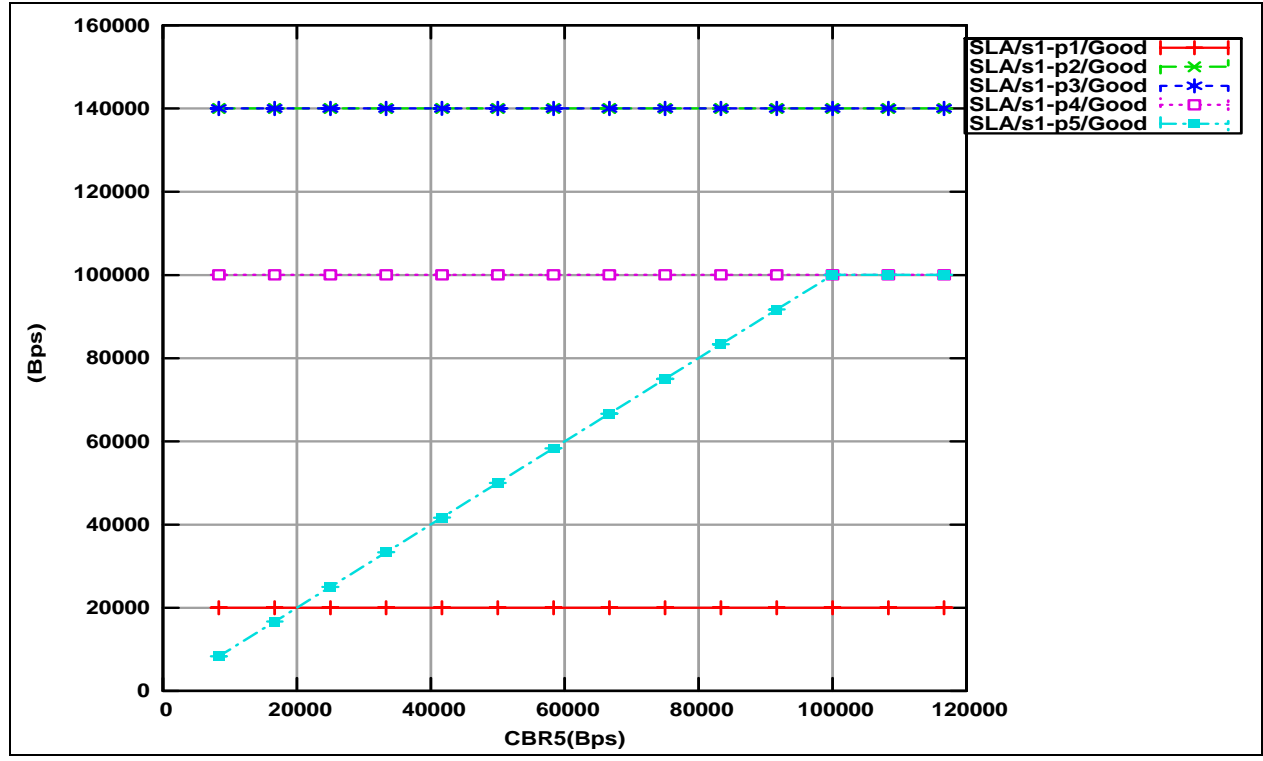

Figure 126 Client1 p1 through p5 goodput Versus p5 throughput

Figure 127 shows client1's priority 5 through priority 8 goodputs. As priority 5 rate increases, the gap at priority 5 decreases which causes less priority 6 upgrades, which in turn causes more priority 6 traffic to be forwarded as priority 6 leaving a smaller gap at priority 6 , which causes more priority 7 to be forwarded as priority 7 leaving a smaller gap at priority 7 , and so on... So for example, for cbr5=40,000 Bps the priority 5 gap is 60,000 Bps. Priority 6 gets to upgrade $60,000 \mathrm{Bps}$, and forward the remaining $80,000 \mathrm{Bps}$ as priority 6 . This leaves a gap of 20,000 Bps at priority 6, which priority 7 uses to get an extra 20,000 Bps rate (total goodput is $100,000+20,000)$. In turn, this leaves no room for priority 8 upgrades. Figure 128 
and Figure 129 show priority 4 through priority 8 upgrade rates and packet losses, respectively, as priority 5 traffic rate increases.

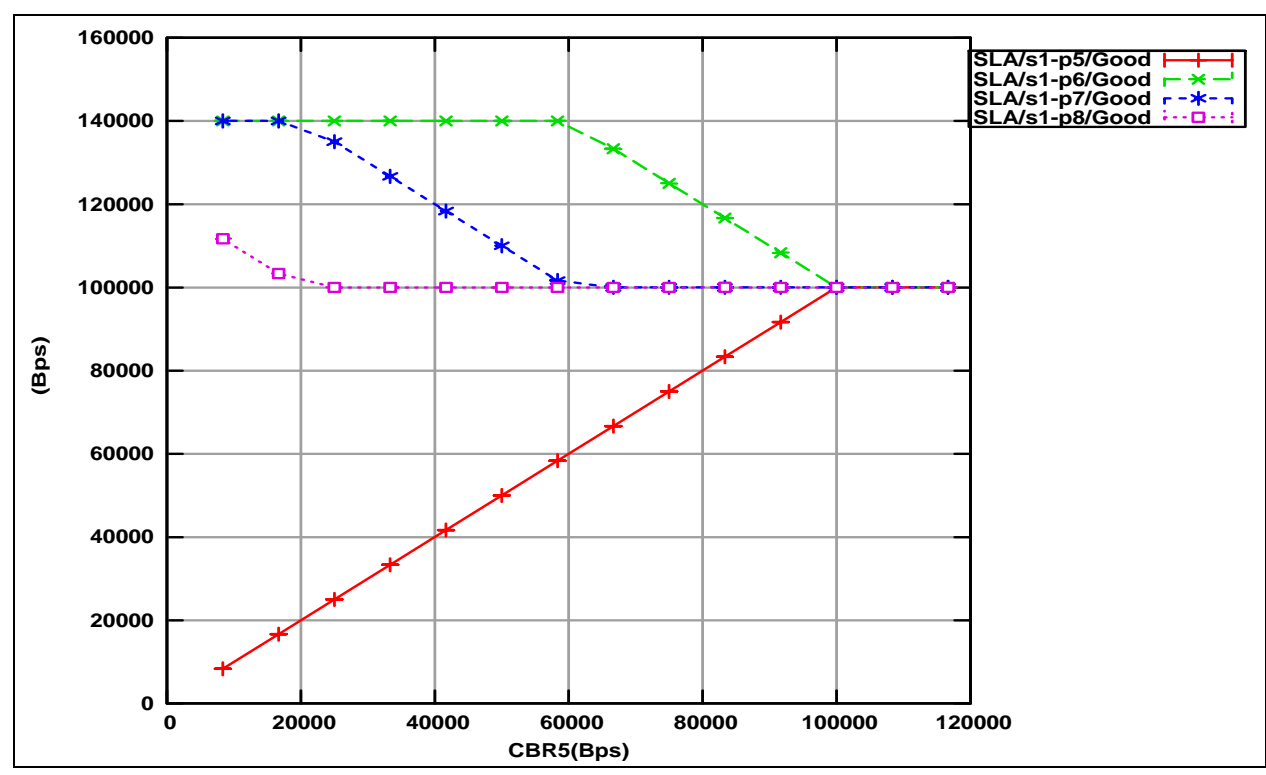

Figure 127 Client1 p5 through p8 goodput Versus p5 throughput

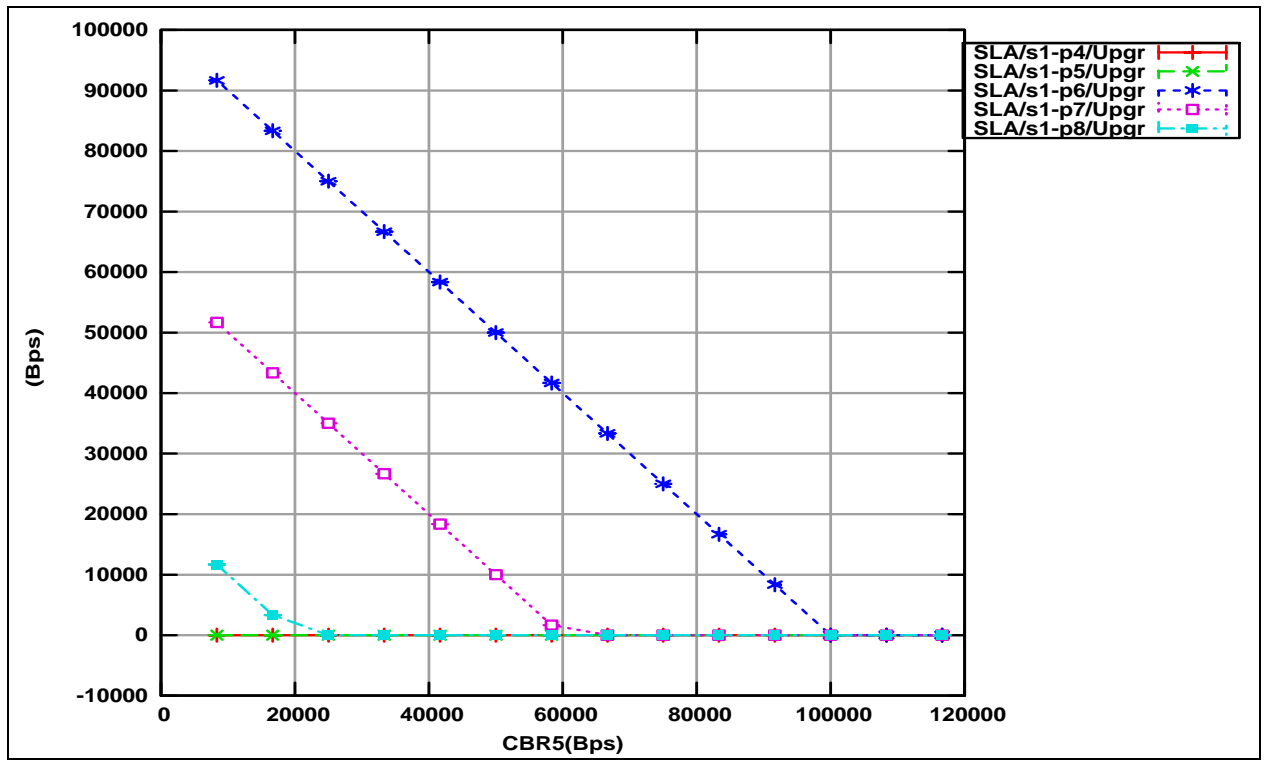

Figure 128 Client1 p4 through p8 upgrades rate Versus p5 throughput 


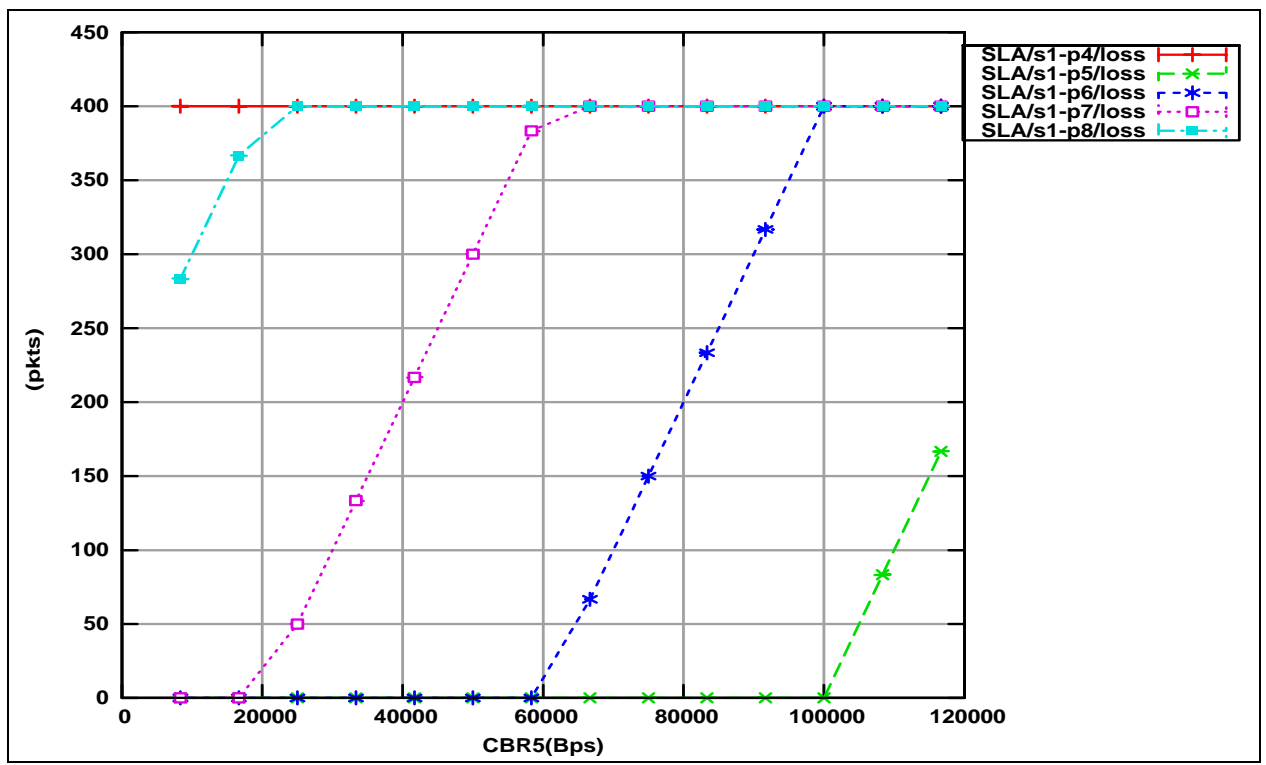

Figure 129 Client1 p4 through p8 packet losses Versus p5 throughput

To re-iterate the previous chapters' findings, we display in Figure 130 and Figure 131 the DS and SLAR behaviors and compare them to the SLA behavior. Plotting more than 5 curves in a single plot is hard to follow; so, we show the DS and SLAR behaviors in separate graphs and compare them to Figure 127 (the SLA goodput). 


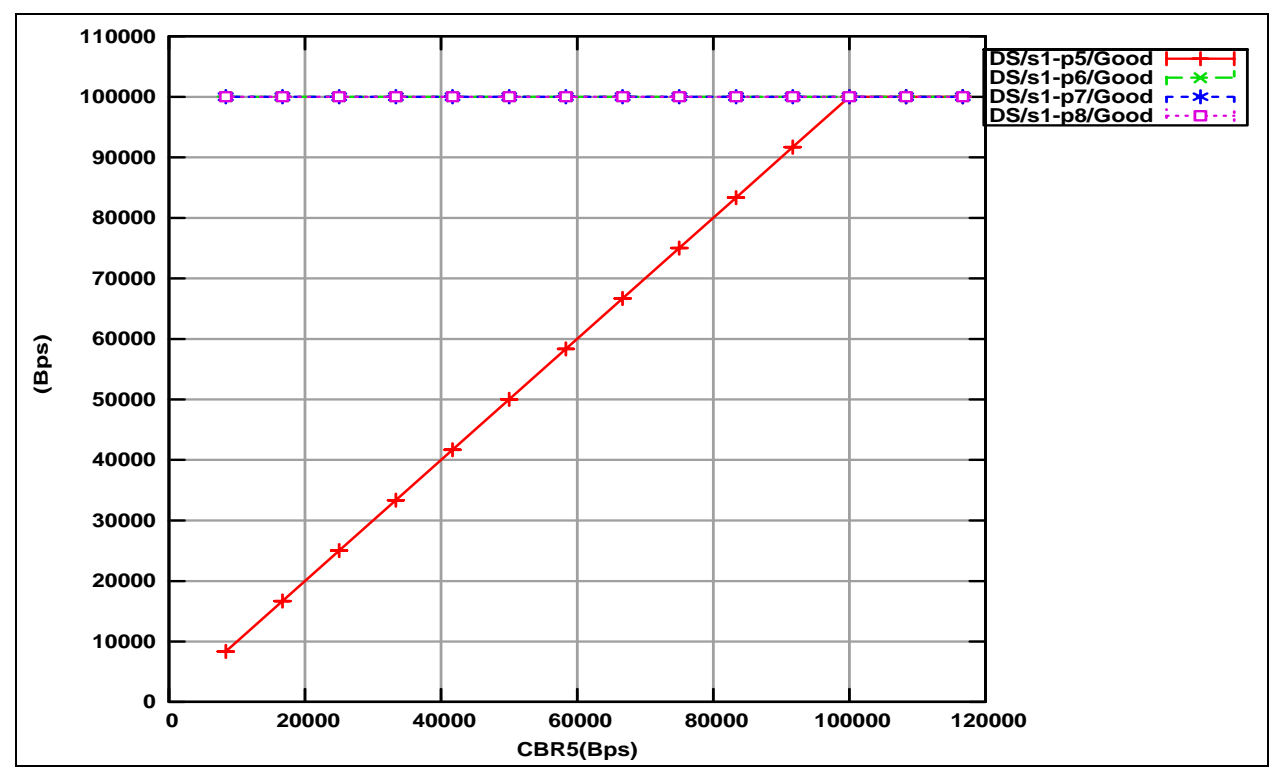

Figure 130 Client1 p5 through p8 goodput Versus p5 throughput

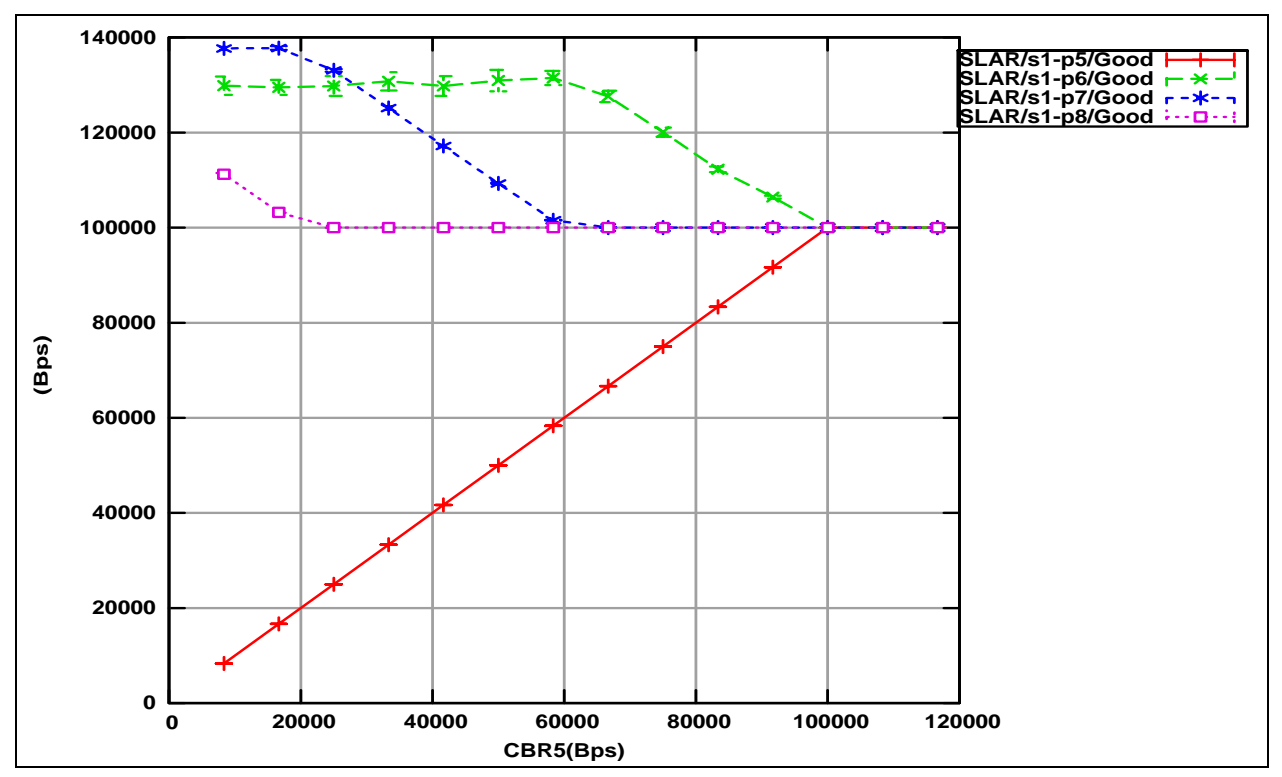

Figure 131 Client1 p5 through p8 goodput Versus p5 throughput

As shown in the above figures, the DS does not allow upgrades so CBR5 has no impact on the other traffic class rates (they are all policed at 100,000Bps). As for the SLAR behavior, although priority 6,7 and 8 classes received better goodput, they did not get the 
same goodput as the SLA model. This is explained by packet reordering which is shown in Figure 132.

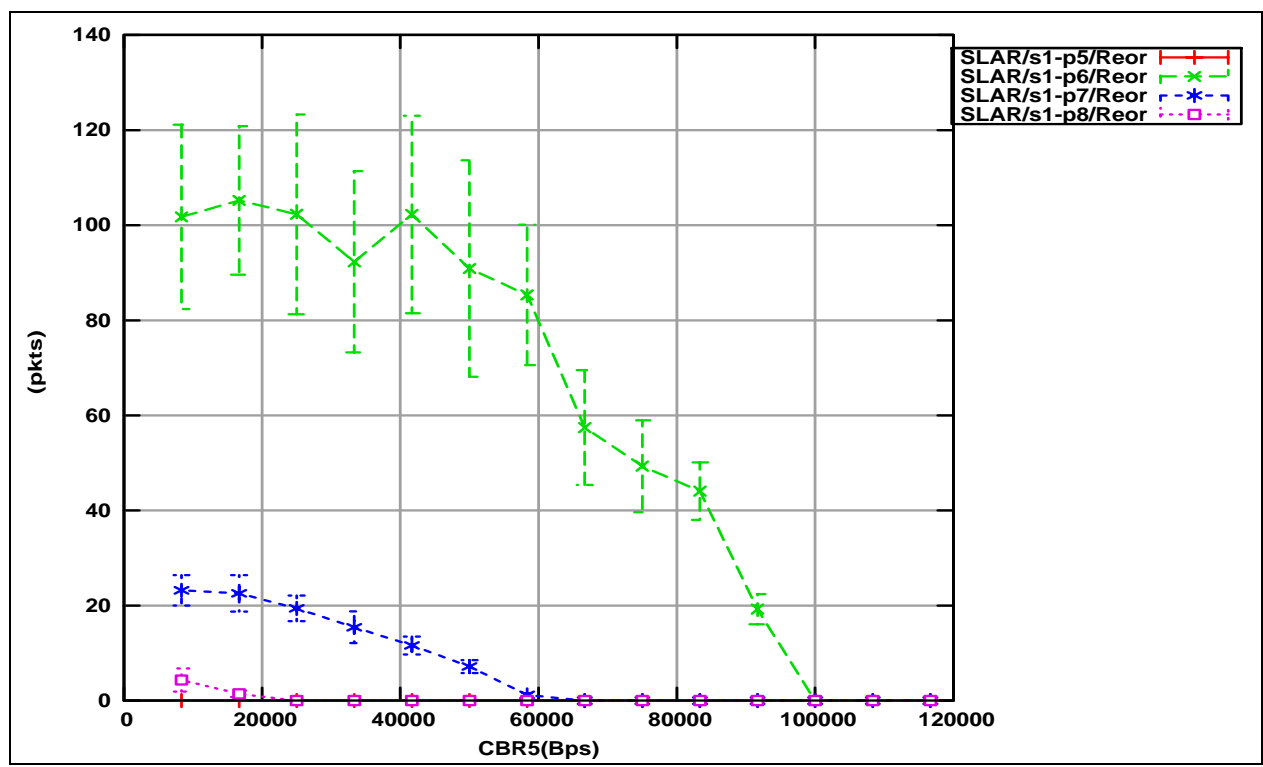

Figure 132 Client1 p5 through p8 packets reordered Versus p5 throughput

To summarize this section's findings, we have shown that the SLA behavior does not depend on the number of traffic classes by generalizing into $\mathrm{N}$ traffic classes, where $\mathrm{N}=8$.

\subsection{TCP Behavior}

In this section, we use the same network setup as described in section 9.1, except that we use a CIR of $882 \mathrm{KBps}$ for the priority 6 background traffic, and we vary the priority 6 background rate from 0 to to $1 \mathrm{MBps}$ to induce congestion at the priority 6 pipe. We also use a CIR of 330KBps for client2's priority 5 traffic in order to create a gap at the priority 5 class. As shown in Table 10, the rest of client 2's policed rates are set to 230,000Bps for all priorities except for priority 1 which is set to $330,000 \mathrm{Bps}$.

With the maximum average TCP rate approximated at $\mathrm{cwnd} / \mathrm{RTT}=$ $20 \times 1,500 / 2 \times 60 \mathrm{~ms}=250 \mathrm{KBps}$, priority 1 traffic gets the full rate since it is policed at 
$330 \mathrm{KBps}$. This creates a gap for the priority 2 traffic, which the latter makes use of to also reach the full throughput. Similarly, priority 3 through priority 5 traffics get this full rate, as shown in Figure 133. This is due to the gap in priority 1 which allows for upgrades at priority 2 through 5 as observed in Figure 134. Notice that priority 1 through priority 5 classes are little affected by the priority 6 background traffic; they are barely impacted as the network gets congested for BG-CBR6 $>800 \mathrm{KBps}$ as also shown in Figure 135, which shows the forward direction delay for each class.

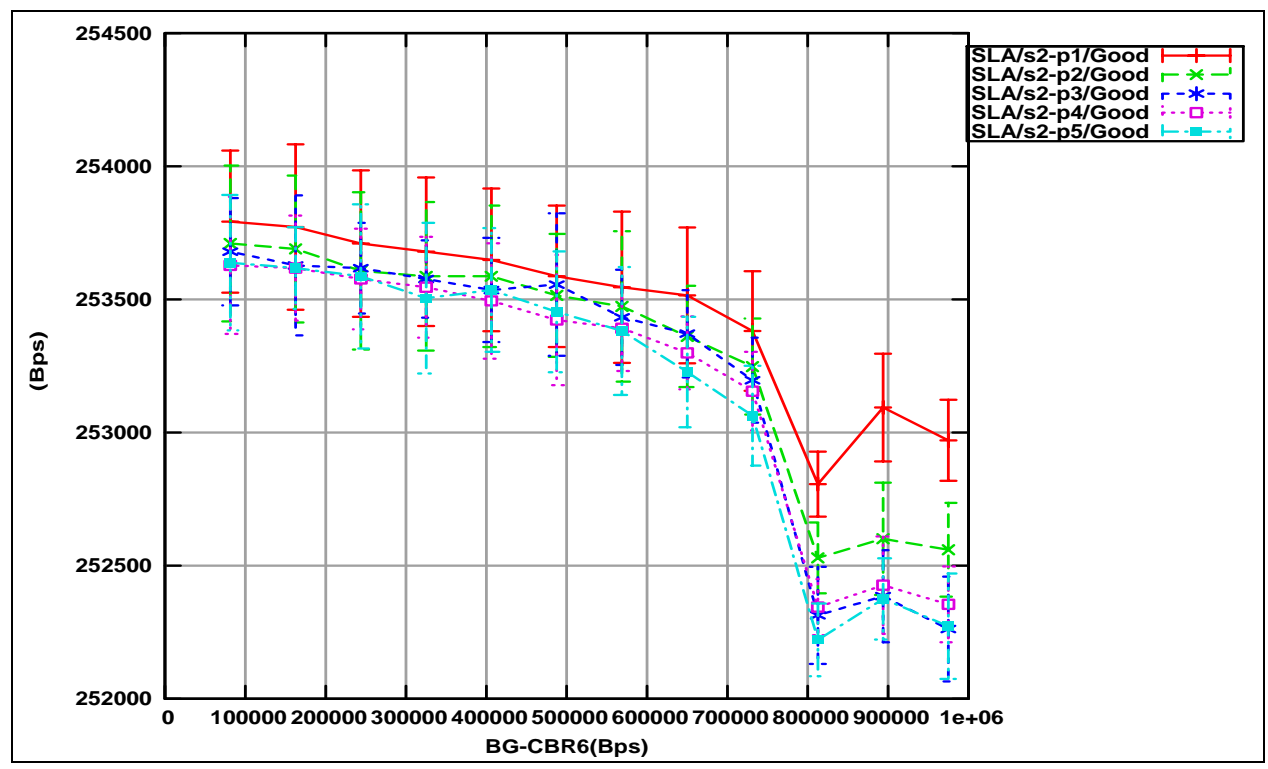

Figure 133 Client2 p1 through p5 goodput Versus p6 background rate 


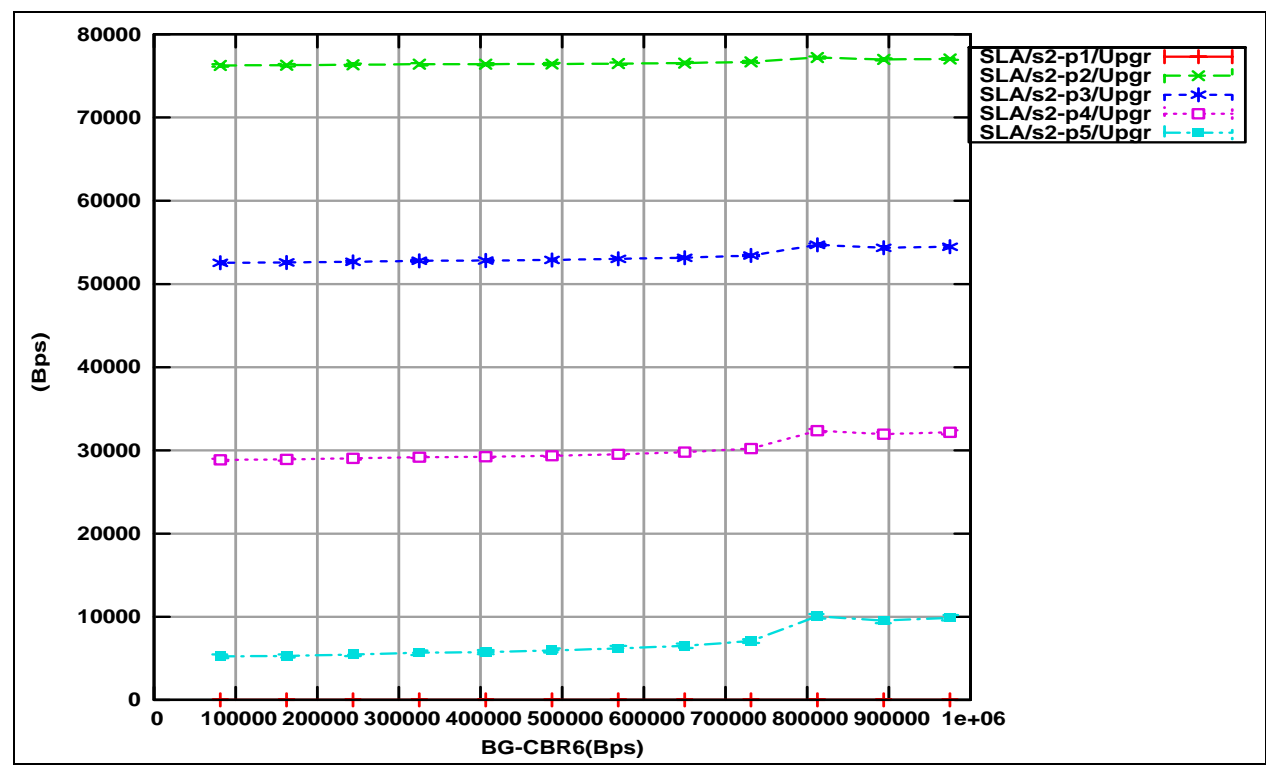

Figure 134 Client2 p1 through p5 upgrade rates Versus p6 background rate

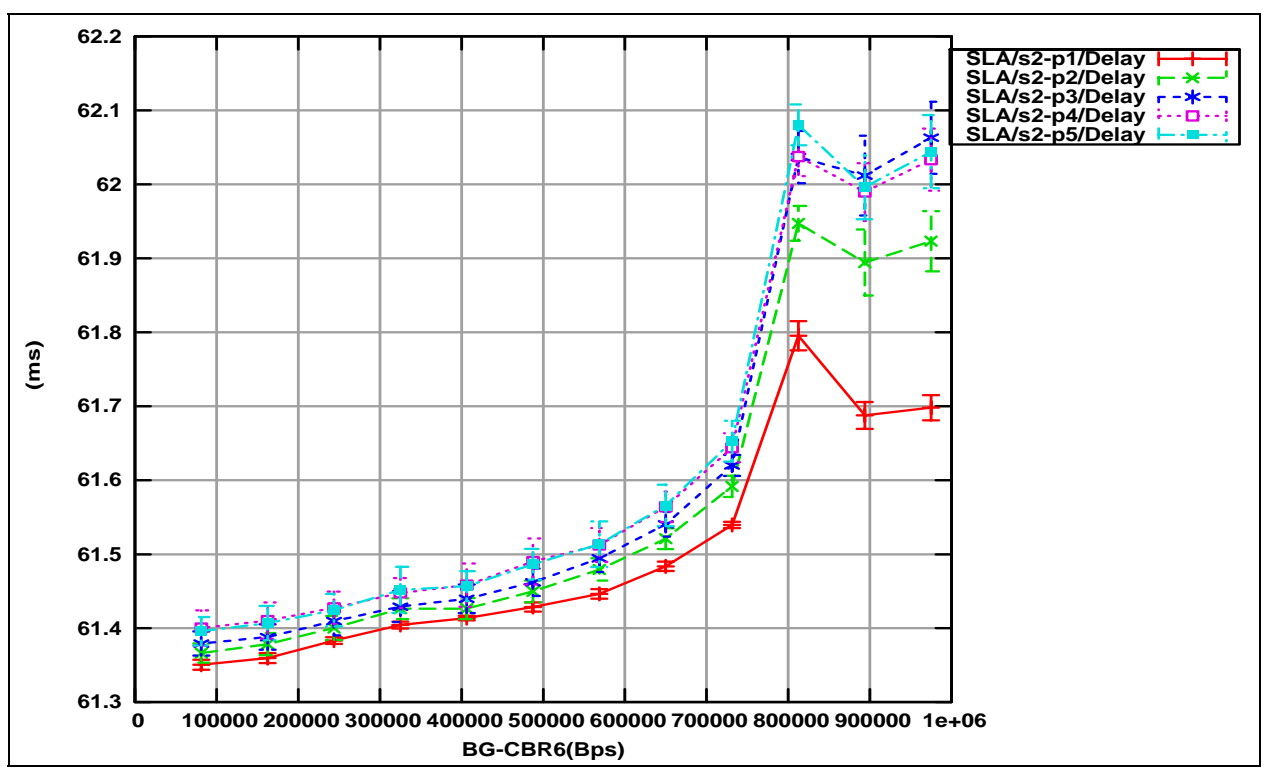

Figure 135 Client2 p1 through p5 end-to-end delay Versus p6 background rate

The priority 6 through priority 8 goodputs are shown in Figure 136. We can see that as the background priority 6 increases, congesting the priority 6 pipe, client 2 's priority 6 TCP reacts to this congestion by dropping its throughput (as also indicated by losses detected as triple duplicate ACK shown in Figure 137). Notice that the priority 8 traffic makes use of the 
gaps created in the upper classes, as a result of dropping their throughput due to congestion, to get the full rate as shown in Figure 138.

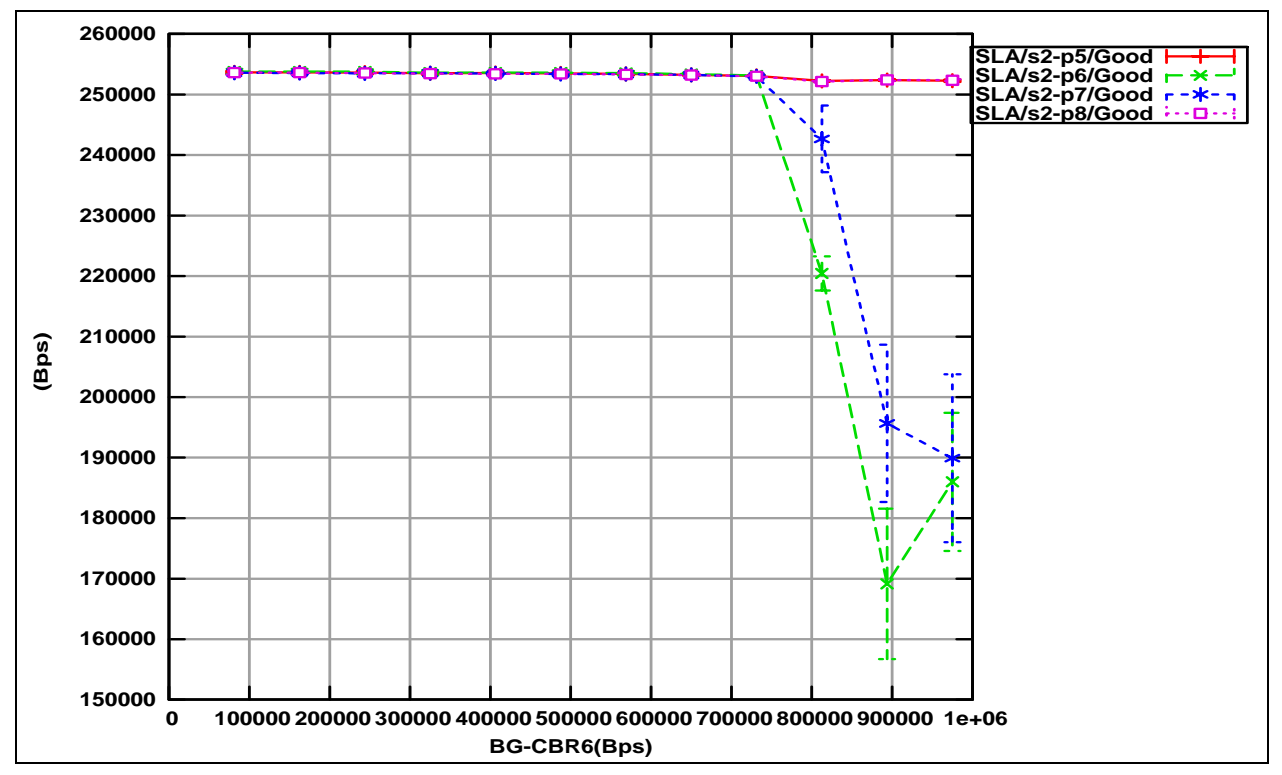

Figure 136 Client2 p5 through p8 goodput Versus p6 background rate

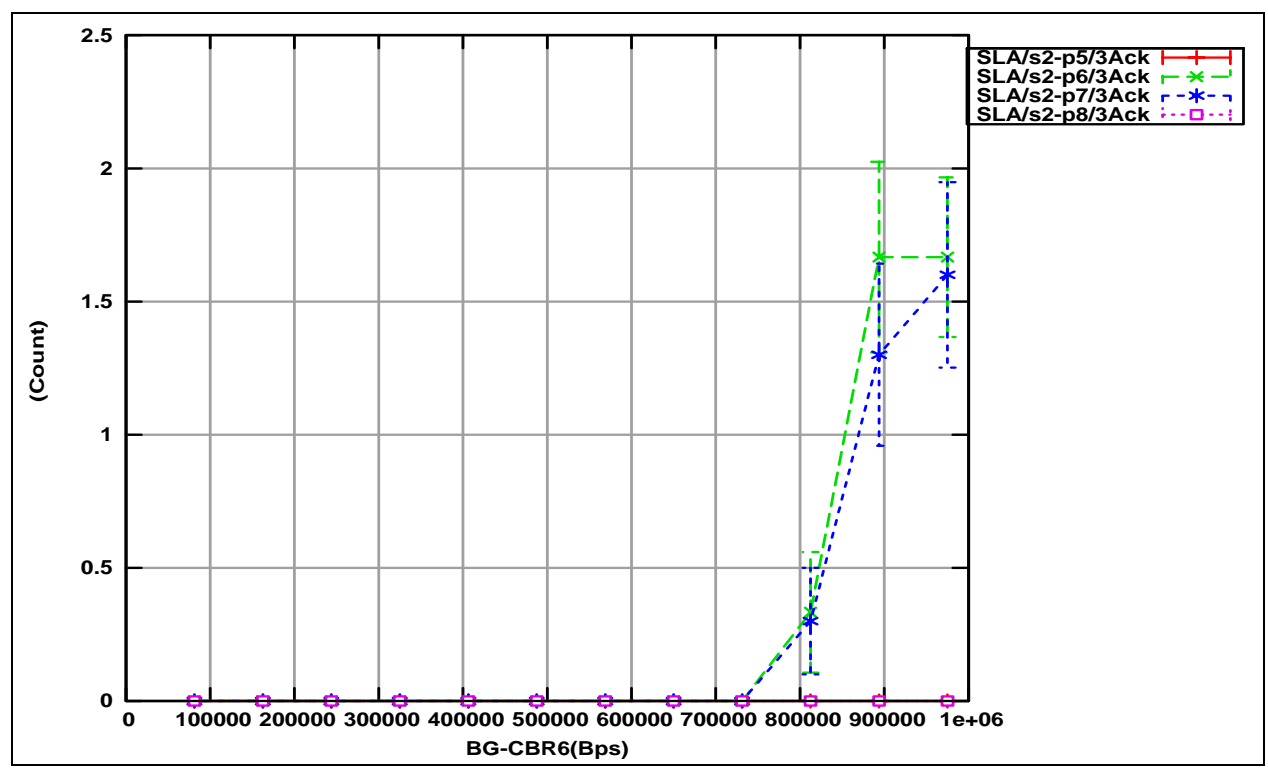

Figure 137 Client2 p5 through p8 3+ duplicate ACKs Versus p6 background rate 


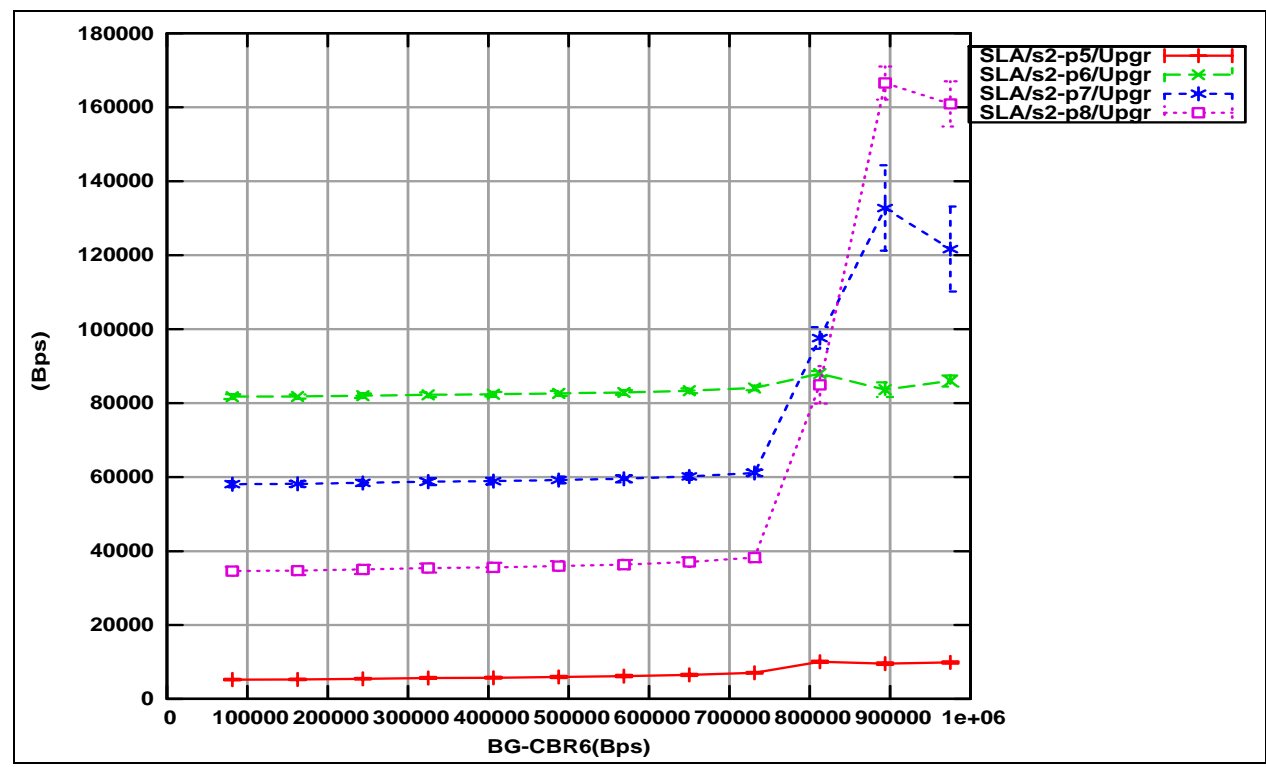

Figure 138 Client2 p5 through p8 upgrade rates Versus p6 background rate

In general, the TCP behavior for the $\mathrm{N}=8$ case is very much in line with our findings in Chapter 8. We can conclude that the SLA behavior does not depend on the number of service classes.

\subsection{Summary and Observations}

In this chapter, we have generalized the 3-tier SLA to an N-tier SLA to show that the QoS components and the reordering solution do not depend on the number of classes. We have investigated both UDP and TCP results for a particular case of $\mathrm{N}=8$, and got similar results as for the $\mathrm{N}=3$ case. 


\section{Chapter 10}

\section{Conclusion and Future Research}

SLAs define the services a carrier provides to the customer: the supported products, the evaluation criteria, the Quality of Service promised, and the penalties assessed if the SLA is not met. It also serves as a differentiator for a Service Provider. So it is no wonder that both customer and provider demand for SLAs is growing, both in number and type of agreement.

In this thesis, we have performed a detailed survey of the latest SLA published papers and classified them into four categories that represent the major research areas in the field: SLA Definition, SLA Management, SLA Monitoring and Reporting, and SLA to QoS controls mapping.

We have proposed a new SLA called "3-tier SLA with automatic class upgrades" that enhances the suggested "triple play" SLA by automatically upgrading lower-class packets to use upper-class gaps or unused bandwidth. We have also presented a solution to the reordering problem that arises due to packet service level demotion or promotion. The 
solution is generic and enables certain Service Level Agreements that rely on upgrading or downgrading customer's traffic.

We suggested a set of QoS components (such as meters, new policers, etc.) that provide the SLA and wrote our own modular $\mathrm{C}++$ classes (replacing the ns- 2 provided diffserv module) that implement the suggested QoS components. We also used a set of TCL scripts to drive various simulations in order to investigate the benefits in terms of throughput (TCP and UDP), delay and loss of the proposed SLA by comparing the simulation performance results of 3 models: 3-tier SLA with plain Diffserv (no automatic upgrades) called DS model, 3-tier SLA with automatic upgrades and without the reordering solution called SLAR model, and finally the proposed 3-tier SLA with automatic upgrades and with the reordering solution called SLA model.

We have demonstrated the benefits of the proposed SLA by comparing the SLA QoS performance under CBR traffic in terms of goodput, delay and packet losses to the DS and SLAR performances and concluded that the SLA model outperforms the other models. We have investigated the effect of the network load on reordering, and showed that the reordering is maximized when the lower class queues are filled and the upper class queues are empty. We have also established that the packet size does not affect the goodput and loss behavior of the SLA model but having larger packet sizes achieved higher goodputs in the SLAR model due to lower reordering; also, as the packet size increases, the end-to-end delay increased due to higher transmission delays. We have concluded that the SLA gives the client the ability to fully benefit from his/her higher classes reserved bandwidths without any reordering in the network. 
We also analyzed the behavior of the SLA under bursty traffic over higher speed links, emulating provider-provider boundary, with lower propagation delay, emulating US East-to-West coast propagation delay. For that, we used IPP arrivals with high coefficient of variation to generate bursty traffic and showed that the SLA behavior did not depend on the traffic type and that the SLA model still outperformed the DS and SLAR models.

We have also demonstrated that the SLA benefit is directly proportional to the upperclasses' gap size, i.e. the bigger the unused bandwidth in the higher classes, the bigger the advantage of the SLA over the DS model for example. Hence, we expect a bigger advantage at the provider-provider boundary where the negotiated rates are expected to be much higher than at the customer-provider boundary.

We also investigated the TCP behavior for the SLA model comparing it with the DS and SLAR models. We showed that lower-class TCP sources benefit from the automatic upgrades (SLAR and SLA) to achieve higher throughput when compared to the non-upgrades model (DS), and that the reordering effect caused by the SLAR model is dampened by the TCP receive buffers. We observed that the SLAR model behaves better than the SLA model under heavy congestion of the lower-class pipe, since the upgraded packets flow on the upper pipe bypassing the congestion in the lower pipe versus the SLA model where upgraded packets flow on the lower-class pipe to maintain the order. We also showed that the TCP throughput is bounded by a maximum rate approximated by cwnd/RTT and that TCP can not make use of upper class gaps larger than this maximum rate. We also claimed that in the DS model, for a client to fully utilize his/her paid-for bandwidth, s/he needs to accurately estimate his RTT in order to avoid tight policing (RTT over-estimated) and over-booking (RTT under-estimated), whereas the SLAR and SLA models provide full usage of the paid 
for bandwidth by upgrading lower class traffic to use the gap in the upper class. We also concluded that the TCP flavor has little to no impact on the upgrade (SLAR or SLA) benefits. We have also shown that the SLA behavior does not depend on the number of traffic classes by generalizing into $\mathrm{N}$ traffic classes, where $\mathrm{N}=8$.

Finally, we have identified some future research items listed herein:

- How do we perform CAC on the proposed SLA?

- In legacy single QoS class SLAs, providers use statistical multiplexing to sell services to a higher number of clients. With full usage of upper classes, how do we charge or sell this SLA so that the provider can offset the statistical multiplexing?

- Can we take advantage of the SLAR property of "better performance during congestion" (refer to Figure 101) in the inner network nodes to upgrade TCP packets to upper classes instead of using tokens?

- We mentioned earlier that TCP can not fully utilize gaps in the upper class (due to approximated max TCP throughput). UDP on the other hand is not bounded to a maximum rate. Are there any drawbacks if we use UDP as a lower class to fill up the gaps created in an upper class TCP source?

- In the TCP RTT section, we showed how the throughput depends on RTT. Could the provider delay packets to lower TCP throughput or could the client change his RTT estimation to raise the TCP throughput?

- Investigate other attractive SLAs that invoke remarking and reordering, like for example upgrading class $m$ to class $n$, or upgrading $m$ classes to the same class of service, time of day related upgrades, etc. 
- Investigate usage of such SLAs by network technologies based on virtual circuit switching like MPLS and ATM. Study the network utilization improvements provided by such automatic class upgrades SLAs versus no upgrades and reserved virtual circuits.

- Investigate the application of such SLAs in $3^{\text {rd }}$ or future generation wireless networks where bandwidth is scarce and expensive, and applications vary from video to text messaging (IMS networks).

- Can we optimize the QoS controls to improve on the performance or overhead? 


\section{Bibliography}

(1) E. Marilly, O. Martinot, S. Betge-Brezetz, G. Delegue, "Requirements for service level agreement management", IP Operations and Management, 2002 IEEE Workshop on, 2002, Pages:57 - 62.

(2) J. Martin, A. Nilsson, "On service level agreements for IP networks", INFOCOM 2002. Twenty-First Annual Joint Conference of the IEEE Computer and Communications Societies. Proceedings. IEEE, Volume: 2 , 23-27 June 2002, Pages:855 - 863 vol.2. charter.html

$$
\text { Internet Traffic Engineering - http://www.ietf.org/html.charters/tewg- }
$$

(4) Realtime Traffic Flow Measurement - Inactive IETF Working Group - RFC2720-2724.

(5) IP Performance Metrics Working group http://ietf.org/html.charters/ippm-charter.html

(6) Remote Network Monitoring - http://ietf.org/html.charters/rmonmibcharter.html

(7) Third Generation Partnership Project- http://www.3gpp.org/

(8) Traffic Engineering for Quality of Service in the Internet at Large http://www.ist-tequila.org/

(9) "Definition of the Differentiated Services Field in the IPv4 and IPv6 headers" - IETF, RFC 2474, June 1998

(10) “An architecture for Differentiated Services” IETF, RFC 2475, December 1998

(11) D. Goderis, "Service Level Specification Semantics and Parameters" IETF Draft-<draft-tequila-sls-00-txt $>$, November 2000.

(12) Adaptive Resource Control for QoS Using an IP based Layered Architecture - http://www-st.inf.tu-dresden.de/aquila/

TeleManagement Forum - http://www.tmforum.org/

“SLA Management Handbook”-TMF GB971, June 2001 
(15) "Definition and usage of SLSs in AQUILA consortium" IETF Draft$<$ draft-salsano-aquila-sls-00.txt $>$ S. Salsano, November 2000.

(16) The Creation and Deployment of End-User Services in Premium IP Networks - http://www.cadenus.org/

(17) R. Garg, H. Saran, R.S. Randhawa, M. Singh, "A SLA framework for QoS provisioning and dynamic capacity allocation", Quality of Service, 2002. Tenth IEEE International Workshop on , 15-17 May 2002, Pages:129 - 137.

(18) R. Chakravorty, I. Pratt, J. Crowcroft, M. D'Arienzo, "Dynamic SLAbased QoS control for third generation wireless networks: the CADENUS extension", Communications, 2003. ICC '03. IEEE International Conference on, Volume: 2, 11-15 May 2003, Pages:938 - 943.

(19) R. Chakravorty, I. Pratt, J. Crowcroft, “A framework for dynamic SLA-based QoS control for UMTS", Wireless Communications, IEEE [see also IEEE Personal Communications], Volume: 10 , Issue: 5 , Oct 2003, Pages:30 - 37.

(20) Akan, B. Baykal, "Dynamic SLA management in cellular DiffServ networks", Telecommunications, 2003. ICT 2003. 10th International Conference on , Volume: 1, 23 Feb.-1 March 2003, Pages:330 - 333 vol.1.

(21) C. Courcoubetis, V.A. Siris, "Managing and pricing service level agreements for differentiated services", Quality of Service, 1999. IWQoS '99. 1999 Seventh International Workshop on , 31 May-4 June 1999, Pages:165 - 173.

(22) Zhenhai Duan, Zhi-Li Zhang, Yiwei Thomas Hou, "Service overlay networks: SLAs, QoS and bandwidth provisioning", Network Protocols, 2002. Proceedings. 10th IEEE International Conference on , 12-15 Nov. 2002, Pages:334 - 343.

(23) S.K. Biswas, S. Ganguly, R. Izmailov, "Path provisioning for service level agreements in Differentiated Services networks", Communications, 2002. ICC 2002. IEEE International Conference on, Volume: 2, 28 April-2 May 2002, Pages:1063 -1068 vol.2.

(24) I. Khalil, T. Braun, "A range-based SLA and edge driven virtual core provisioning in DiffServ-VPNs", Local Computer Networks, 2001. Proceedings. LCN 2001. 26th Annual IEEE Conference on , 14-16 Nov. 2001, Pages:12 - 21.

D. Goderis, Y. T'joens, C. Jacquenet, G. Memenios, G. Pavlou, R. Egan, D. Griffin, P. Georgatsos, L. Georgiadis, P. Van Heuven, "Service Level Specification Semantics, Parameters and negotiation requirements", internet draft drafttequila-sls-01.txt, June 2001. 
(26) D. Goderis, Y. T'joens, C. Jacquenet, G. Memenios, G. Pavlou, R. Egan, D. Griffin, P. Georgatsos, R. Rajan, S. Salsano, L. Georgiadis, P. Van Heuven, "Service Level Specification and Usage Framework", draft-manyfolks-sls-framework00.txt, Oct. 2000.

(27) Luo Tie, Meng Luoming, "SLA foundation template library: reusablecomponent repository for SLA", Communication Technology Proceedings, 2003. ICCT 2003. International Conference on , Volume: 2 , April 9 - 11, 2003, Pages:1739- 1743.

(28) Jong-Tae Park, Jong-Wook Baek, James Won-ki, "Management of Service Level Agreements for multimedia internet service using a utility model", IEEE communications magazine, May 2001, pp100-106.

(29) V. Paxson, G. Almes, J. Mahdavi, M. Mathis, "Framework for IP Performance Metrics", RFC 2330, May 1998

(30) J. Mahdavi, V. Paxson, "IPPM Metrics for Measuring Connectivity", RFC 2678, September 1999

(31) G. Almes, S. Kalidindi, M. Zekauskas, "A One-way Delay Metric for IPPM”, RFC 2679, September 1999

(32) G. Almes, S. Kalidindi, M. Zekauskas, "A One-way Packet Loss Metric for IPPM", RFC 2680, September 1999

(33) G. Almes, S. Kalidindi, M. Zekauskas, “A Round-trip Delay Metric for IPPM", RFC 2681, September 1999

(34) D.D. Lamanna, J. Skene, W. Emmerich, "SLAng: A language for defining service level agreements", Distributed Computing Systems, 2003. FTDCS 2003. Proceedings. The Ninth IEEE Workshop on Future Trends of , 2003, Pages:100 - 106.

(35) Thi Mai Trang Nguyen, N. Boukhatem, Y.G. Doudane, G. Pujolle, "COPS-SLS: a service level negotiation protocol for the Internet", Communications Magazine IEEE, Volume: 40 , Issue: 5 , May 2002, Pages:158 - 165.

(36) D. Durham, Ed., J. Boyle et al. "The COPS (Common Open Policy Service) Protocol”, RFC 2748, Jan 2000.

(37) The Frame Relay Forum, http://www.mplsforum.org/frame/

(38) J. Shin, J. Kim, C. Jay Kuo, "Quality of service mapping mechanism for packet video in differentiated services network", Packet Video Workshop, May 2000.

(39) S. D’Antonio, M. Esposito, M. Gargiulo, S. P. Romano, G. Ventre, “A Component-based approach to SLA Monitoring in premium IP networks", International 
Workshop on Inter-domain Performance and Simulation, http://www.ist-intermon.org/, Feb 2003.

(40) C. Diot, G. Iannaccone, A. Markopoulou, C. Chuah, S. Bhattacharyya, "Service Availability in IP Networks", Sprint ATL research report, http://ipmon.sprint.com/pubs_trs/webpages/ATLRR.php, July 2003.

(41) R. Sprenkels, A. Pras, "Service Level Agreements", Internet NG, Deliverable D2.7, April 2001

(42) Authentication, Authorization and Accounting Working Group http://www.ietf.org/html.charters/aaa-charter.html

(43) N. Brownlee, “Traffic Flow measurement: Meter MIB”, RFC 2720, October 1999.

(44) Open source reference, e.g. http://www.opensource.org

(45) K. McColghrie, M. Rose, "Management Information Base for Network Management of TCP/IP based internets: MIB-II”, RFC 1213, March 1991.

(46) S. Waldbusser, "Token Ring Extensions to the Remote Network Monitoring MIB”, RFC 1513, Septemeber 1993.

(47) S. Walbusser, "Remote Network Monitoring Management Information Base”, RFC 1757, February 1995

(48) S. Waldbusser, "Remote Network Monitoring Management Information Base Version 2", RFC 2021, January 1997

(49) A. Bierman, R. Iddon, "Remote Network Monitoring MIB Protocol Identifiers", RFC 2074, January 1997.

(50) D. Goderis, D. Griffin, C. Jacquenet, G. Pavlou, "Attributes of a Service Level Specification (SLS) template", <draft-tequila-sls-03.txt>, Work in progress, October 2003.

(51) S. Salsano, F. Ricciato, M. Winter, G. Eichler, A. Thomas, F. Fuenfstueck, T. Ziegler, C. Brandauer, "Definition and usage of SLSs in the AQUILA consortium", <draft-salsano-aquila-sls-00.txt>, Expired Internet Draft, November 2000. atm.html Wiltel: http://www.wiltel.com/services/enhanceddata/atm/r_sla- 
(53) R. Rajan, E. Celenti, S. Dutta, "Service Level Specification for Interdomain QoS Negotiation”, < draft-somefolks-sls-00.txt>, Expired Internet Draft, November 2000.

(54) T.M.T. Nguyen, N. Boukhatem, Y. El Mghazli, N. Charton, L.N. Hamer, G. Pujolle, "COPS-PR Usage for SLS Negotiation (COPS-SLS)", < draft-nguyenrap-cops-sls-03.txt>, Expired Internet Draft, July 2002.

(55) A. Dutta-Roy, "The cost of quality in Internet-style networks", Spectrum, IEEE , Volume: 37 , Issue: 9 , Sept. 2000, Pages:57 - 62

(56) E. Marilly, O. Martinot, H. Papini, D. Goderis, "Service level agreements: a main challenge for next generation networks", Universal Multiservice Networks, 2002. ECUMN 2002. 2nd European Conference on , 8-10 April 2002, Pages:297 - 304

(57) E. Bouillet, D. Mitra, K.G. Ramakrishnan, "The structure and management of service level agreements in networks", Selected Areas in Communications, IEEE Journal on, Volume: 20 , Issue: 4 , May 2002, Pages:691 - 699.

(58) M. C. Chan, Y.J. Lin, X. Wang, "A scalable monitoring approach for service level agreements validation", Proceedings of the 2000 International Conference on Network Protocols, November 2000

(59) T. Braun, L. Ru, G. Stattenberger, "An AAA architecture extension for providing differentiated services to mobile IP users", Computers and Communications, 2001. Proceedings. Sixth IEEE Symposium on , 3-5 July 2001, Pages:472 - 478

(60) M. Mellia, C. Casetti, G. Mardente, M.A. Marsan, "An analytical framework for SLA admission control in a DiffServ domain", Global Telecommunications Conference, 2002. GLOBECOM '02. IEEE, Volume: 3 , 17-21 Nov. 2002, Pages:2563 - 2567 vol.3

(61) Y. Elovici, Y. Ben-Shimol, A. Shabtai, "Per-packet pricing scheme for IP networks", Telecommunications, 2003. ICT 2003. 10th International Conference on , Volume: 2 , 23 Feb.-1 March 2003, Pages:1494 - 1500 vol.2

(62) A. Iwata, N. Fujita, "A hierarchical multilayer QoS routing system with dynamic SLA management", Selected Areas in Communications, IEEE Journal on , Volume: 18 , Issue: 12 , Dec. 2000, Pages:2603 - 2616

(63) Wei Hong, Meng Luoming, Qiu Xuesong, "End-to-end service management architecture and information model for MPLS VPN with dynamic SLA management",

Communication Technology Proceedings, 2003, ICCT 2003, International Conference on , Volume: 2 , 9-11 April 2003, Pages:1553 - 1557 vol.2 
(64) M. D' Arienzo, M. Esposito, S.P. Romano, G. Ventre, “Automatic SLA management in SLA-aware architecture", Telecommunications, 2003. ICT 2003. 10th International Conference on , Volume: 2 , 23 Feb.-1 March 2003, Pages:1402 - 1406 vol.2

(65) B. Statovci-Halimi, A. Halimi, K. Hendling, H.R. van As, "A dynamic SLA management approach for MPLS networks", Computers and Communication, 2003. (ISCC 2003). Proceedings. Eighth IEEE International Symposium on, 30 June-3 July 2003, Pages:945 - 950

(66) B. Statovci-Halimi, A. Halimi, K. Hendling, H.R. van As, "A framework for dynamic SLA management under heterogeneous traffic conditions in MPLS networks", Performance, Computing, and Communications Conference, 2003. Conference Proceedings of the 2003 IEEE International , 9-11 April 2003, Pages:217 224

(67) K. Pulakka, "Controlling of satisfaction of the end-users and profits of the ISPs in the DS enabled Internet", Communication Systems, 2002. ICCS 2002. The 8th International Conference on , Volume: 1 , 25-28 Nov. 2002, Pages:138 - 144 vol.1

(68) C.M. Leung, J.A. Schormans, "Measurement-based end to end latency performance prediction for SLA verification", Computer Communications and Networks, 2002. Proceedings. Eleventh International Conference on , 14-16 Oct. 2002, Pages:412 417

(69) R. Haddad, "SLA TO CONTROLS MAPPING IN DIFFERENTIATED SERVICES”, Masters Thesis, May 2000.

(70) S. Bohacek, J.P. Hespanha, J. Lee, C. Lim, K. Obraczka "TCP-PR: TCP for Persistent Packet Reordering", Proceedings of the 23rd International Conference on Distributed Computing Systems, May 2003.

(71) S. Blake, D. Black, M. Carlson, E. Davies, Z. Whang, and W. Weiss “An architecture for differentiated services”, RFC 2475, 1998.

(72) J. Heinanen, F. Baker, W. Weiss, J. Wroclawski "Assured Forwarding PHB Group”, RFC 2597, June 1999.

(73) E. Blanton and M. Allman, "On Making TCP More Robust to Packet Reordering”, ACM Computer Commnication Review, 32(1), 2002.

(74) V. Paxson, "End-to-end Internet Packet Dynamics", IEEE/ACM Transactions on Networking, 7(3):277-292, 1999. 
(75) D. Minoli and E. Minoli, "Delivering Voice over IP Networks", John Wiley \& Sons, 1988.

(76) V. Jacobson, RFC 1144 “Compressing TCP/IP Headers for LowSpeed Serial Links", February 1990.

(77) W. Simpson, RFC 1661 "The Point-to-Point Protocol (PPP)”, July

1994.

(78) J. Bellardo, S. Savage, "Measuring Packet Reordering", Proceedings of the second ACM SIGCOMM Workshop on Internet measurement, November 2002.

(79) J. C. R. Bennett, C. Partridge, N. Shectman, "Packet Reordering is not Pathological Network Behavior", IEEE/ACM Transactions on Networking (TON), Volume 7 Issue 6, December 1999.

(80) M. Laor, L.Gendel, "The effect of packet reordering in a backbone link on application throughput", Network, IEEE, Volume 16, Issue 5, Sept.-Oct. 2002 Page(s):28 - 36 .

(81) S. Ladha, S. Baucke, R. Ludwig, P. Amer, "On Making SCTP Robust to Spurious Retransmissions", ACM SIGCOMM Computer Communication Review, April 2004.

(82) V. Jacobson, "Recommendations on Queue Management and Congestion Avoidance in the Internet", RFC 2309, April 1998.

(83) W. Fischer, K. Meier-Hellstern, "The Markov-Modulated Poisson Process (MMPP) cookbook”. Performance Evaluation, 18:149-171, 1992.

(84) X. Moundrouidou, H. Perros, "Performance Evaluation of an Optical Burst Switching Scheme for Grid Networks", GridNets 2005

(85) H. Perros, "Computer Simulation techniques: the definitive introduction" available at http://www.csc.ncsu.edu/faculty/perros/simulation.pdf. 2003.

(86) G. Neglia, G. Bianchi, “Is TCP Packet Reordering Always Harmful?”, Proceedings of the The IEEE Computer Society's 12th Annual International Symposium on Modeling, Analysis, and Simulation of Computer and Telecommunications Systems (MASCOTS'04) - Volume 00, Pages: 87 - 94

(87) A. Feroz, S. Kalyanaraman, A. Rao, "A TCP-Friendly Traffic Marker for IP Differentiated Services", in Proc. IWQoS'2000, Pittsburgh, PA, June 2000, pp. 3548. 
(88) W. Stevens, "TCP Slow Start, Congestion Avoidance, Fast Retransmit, and Fast Recovery Algorithms", RFC 2001, January 1997.

(89) "Berkley TCP evolution from 4.3 Tahoe to 4.3 Reno", in Proc $18^{\text {th }}$ IETF, Vancouver, Canada, August 1990.

(90) T. V. Lakshman, U. Madhow "The performance of TCP/IP for networks with high bandwidth-delay products and random loss", IEEE/ACM Transactions on Networking (TON), Volume 5 Issue 3, June 1997. 